\title{
Atomic-scale transport in graphene: the role of localized defects and substitutional doping
}

\author{
Dissertation \\ zur Erlangung des mathematisch-naturwissenschaftlichen Doktorgrades \\ "Doctor rerum naturalium" \\ der Georg-August-Universität Göttingen
}

im Promotionsprogramm ProPhys

der Georg-August-University School of Science (GAUSS)

vorgelegt von

\section{Philip Willke}

aus Seesen

Göttingen 2016 


\section{Betreuungsausschuss}

Dr. Martin Wenderoth

IV. Physikalisches Institut, Georg-August-Universität Göttingen

Prof. Dr. Claus Ropers

IV. Physikalisches Institut, Georg-August-Universität Göttingen

\section{Mitglieder der Prüfungskommission}

Referent: Dr. Martin Wenderoth

IV. Physikalisches Institut, Georg-August-Universität Göttingen

Korreferent: Prof. Dr. Konrad Samwer

I. Physikalisches Institut, Georg-August-Universität Göttingen

Korreferent: Prof. Dr. Rolf Möller

Fakultät für Physik und Center for Nanointegration Duisburg-Essen (CeNIDE), Universität Duisburg-Essen

\section{Weitere Mitglieder der Prüfungskommission}

Prof. Dr. Claus Ropers

IV. Physikalisches Institut, Georg-August-Universität Göttingen

Prof. Dr. Stefan Mathias

I. Physikalisches Institut, Georg-August-Universität Göttingen

Prof. Dr. Hans Christian Hofsäss

II. Physikalisches Institut, Georg-August-Universität Göttingen

Prof. Dr. Christian Jooß

Institut für Materialphysik, Georg-August-Universität Göttingen

Tag der mündlichen Prüfung: 08.12.2016 




\section{Introduction}

The continuous progress in device miniaturization demands a thorough understanding of the electron transport processes involved. While device fabrication has made enormous advances in scaling down device dimensions, e.g. a transistor down to less than 10 nanometers [70], this length scale remains difficult to probe experimentally, at least in transport experiments.

The role of defects - discontinuities in the perfect and translational invariant crystal lattice - is here of particular interest. They are a double-edged sword being able to alter a material's properties in a positive or a negative way.

On the one hand they can be utilized to design devices and their properties: for instance, by introducing foreign atoms into a crystal lattice, it can be doped and the Fermi level can be adjusted [216]. In case of the giant magnetoresistance,[5, 62] the electric resistance of a system of thin magnetic layers changes depending on their magnetization. A third example are nitrogen-vacancy centers in diamond that are a candidate for quantum computing [133].

On the other hand defects introduce additional sources of scattering into the sample system. This can be an unwanted byproduct, especially for designing a device with high electron mobility. This is often discussed in case of graphene, the material studied in the framework of this thesis. While electron mobilities in graphene have a high intrinsic limit of $200,000 \mathrm{~cm}^{2} / \mathrm{Vs}$ [25], large scale graphene is still limited by the interaction with defects and the underlying substrate [80].

Often a combination of spatially resolving techniques such as electron microscopy or scanning probe microscopy along with transport measurements is used to access the properties of local scatterers $[44,76,181,210,220]$. This allows to connect the local structure of a sample along with its behavior in transport. However, probing both quantities simultaneously on a microscopic scale is indubitably beneficial for connecting local defect structures with local electron flow and voltage drop.

Several experimental approaches were aiming to achieve this in the past. Electron microscopy [87] and photoemission spectroscopy [93] have both been used to image properties connected to electron transport. However, scanning probe methods are much more represented. Atomic force microscopy (AFM) based techniques such as conductive AFM [179] and microwave interference microscopy [114] as well as the scanning single electron transistor [119] have been successfully used to study mesoscopic transport in the past. 


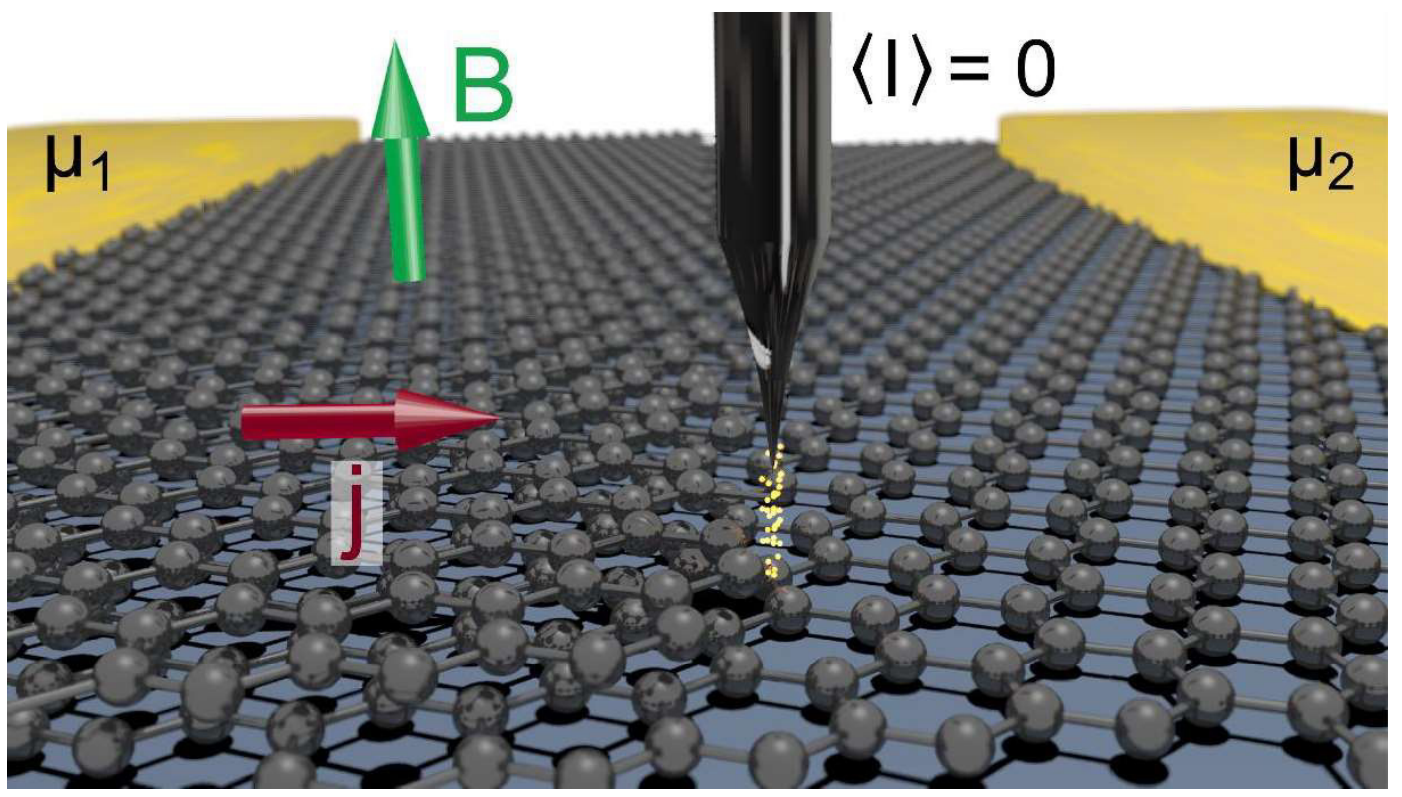

Figure 0.1: Scanning tunneling potentiometry on graphene. As in conventional scanning tunneling microscopy, the sample can be mapped with atomic resolution. In addition, a cross current with current density $j$ is flowing through the sample via two contacts at potential $\mu_{1}$ and $\mu_{2}$. By compensating a net tunneling current $\langle\mathrm{I}\rangle=0$ on the side of the tip, the voltage drop can be mapped across the sample giving insight into the scattering processes involved. In addition, a magnetic field $B$ can be applied to study magnetotransport. Here, the interface between a bilayer graphene (left) and monolayer graphene (right) is sketched, one of the local defects studied in the course of this thesis.

A technique with ultimate spatial resolution down to the atomic scale is scanning tunneling potentiometry (STP), first introduced by Muralt and Pohl in 1986 [125]. The experimental setup is sketched in Figure 0.1. A scanning tunneling microscope (STM) is used to map the sample surface down to atomic resolution. Additionally, a lateral current is flowing through the sample. The voltage drop across the surface can be sensed by adjusting the scanning tip to the same potential as the sample at the position of the tip, analog to a potentiometer. Since only the surface potential can be probed, STP demands ${ }^{1} 2 \mathrm{D}$-conductors or thin films [21, 59, 162].

Consequently, this method regained interest along with the rise of graphene [29, $30,80,142,193,201]$ and other 2D-conductors [11]. Moreover, 2D materials and topological insulators are systems of high interest and rich physics making STP a favorable tool to study the transport behavior down to the atomic scale.

A versatile STP implementation previously developed in our group is able to measure the local voltage drop in a standard STM setup with only minor changes [41], therefore being able to operate at different temperatures as well as magnetic fields.

\footnotetext{
1 Techniqually, bulk materials can also be probed. Then however, surface and bulk transport channels have to be separated. Additionally, if a defect is studied, it must be translational invariant across the whole sample so that electrons cannot simply flow "around" it.
} 
Homoth et al. showed that on a scale of several micrometers down to a few nanometers, the voltage drop as well as the current flow is governed by the interplay between defect-free terraces and monatomic steps [71, 72]. While this work was conducted on a Si(111)- $(\sqrt{3} \times \sqrt{3})$-Ag surface, Druga used our STP approach to study the sheet resistance and defects in graphene [42]. In agreement with others [80], he demonstrated that local defects such as steps and interfaces contribute significantly to the total voltage drop. Moreover, both defect resistances as well as local sheet resistances can vary quite severely locally, leading to an inhomogeneous electron flow.

In the first result part of this thesis (chapter 3) we use scanning probe methods to investigate the transport properties of localized defects in graphene.

Besides a pure characterization of the voltage drop at local defects, revealing the underlying scattering mechanism is of course the subject of interest. Using STP this has up to now only been achieved by analyzing the magnitude of the measured voltage drop. For example, Ji et al. showed that the voltage drop at the interface between monolayer and bilayer graphene (as depicted in Figure 0.1) is significantly larger than that for a monolayer sheet crossing an underlying substrate step [80]. Consequently, they attributed the higher voltage drop to an electronic transition due to the wave function mismatch at the interface. Similar approaches have addressed the scattering behavior in graphene at substrate steps of different height [108] as well as grain boundaries [30]. ${ }^{2}$

In section 3.2, we present an STP-study focusing on the local voltage drop at these graphene monolayer-bilayer junctions. Its content is published as Ref. [201], "Spatial extent of a Landauer residual-resistivity dipole in graphene quantified by scanning tunnelling potentiometry". Here, we use STP to show that the voltage drop at this particular defect is not located strictly at its topographic position, but extends spatially up to a few nanometers into the bilayer side. Additionally, different scattering centers of the junction can be disentangled. Thus, we can show that the exact location of the voltage drop with respect to the defect gives additional insight into the underlying scattering mechanism. ${ }^{3}$

In macroscopic transport experiments the electrical resistance of a sample is usually measured as a function of an external parameter like the charge carrier concentration, a magnetic field or temperature, since a scattering mechanism often leaves a unique fingerprint as a function of such parameters. Measuring defect resistances locally as a function of temperature or magnetic field is another subject of this thesis.

\footnotetext{
${ }^{2} \mathrm{An}$ in-depth introduction to the topic is given in section 3.1 .

${ }^{3}$ Parts of this experiment have already been conducted within the scope of the PhD thesis of $\mathrm{Dr}$. Thomas Druga [42] and my own master thesis [199]. For an in-depth discussion on which parts have been conducted within the scope of this PhD-thesis, see section "Darstellung der wissenschaftlichen Zusammenarbeit" on page 163.
} 
In section 3.3, we present a local transport study using Kelvin probe force microscopy. Its content is published as Ref. [203], "Local transport measurements in graphene on $\mathrm{SiO}_{2}$ using Kelvin probe force microscopy". Here we study the variation of the local sheet resistance of graphene on $\mathrm{SiO}_{2}$ under ambient conditions and as a function of temperature in a range of $20^{\circ} \mathrm{C}-100^{\circ} \mathrm{C}$. Additionally, we resolve the defect resistance of a folded wrinkle for which a temperature-independent model yields the best fit to the data. Thus, we suggest a scattering mechanism due to the interlayer tunneling between graphene layers, different from transport on the pristine graphene sheets.

In section 3.4, we introduce a new magnetic field STP setup. The content of this section is published in Ref. [202], "Magnetotransport on the nanoscale". Here, we study the local sheet resistance and defect resistance as a function of magnetic field up to $6 \mathrm{~T}$. In addition, we are able to extract the charge carrier concentration locally evaluating the change in electric fields similar to the macroscopic Hall effect. We find the resistance of localized defects such as steps and monolayer-bilayer junctions along with their respective underlying scattering mechanisms to be independent on the magnetic field.

In the second part of this thesis in chapter 4 we investigate the properties of substitutional doping atoms in graphene.

As discussed above, defects can be used for electronic band engineering, in particular via atomic doping. Foreign atoms have been used in the past already to change the charge carrier concentration in graphene. It has been first shown by Zhao et al. that introducing substitutional nitrogen (boron) atoms into the graphene sheet leads to n-doping (p-doping) [216, 217]. However, while the charge carrier concentration is in this way nicely tunable, the presence of additional scatterers reduces the electric conductivity. Rein et al. observed that introducing nitrogen atoms into the growth process not only increases the sheet resistance in graphene, but also leads to enhanced phase coherent scattering effects at low temperatures, that is weak localization [152]. The study of graphene doping via foreign ion implantation is the second subject treated in the framework of this thesis. An example is given in Figure 0.2 where we show a single substitutional nitrogen defect incorporated into graphene on silicon carbide ( $\mathrm{SiC}$ ) and imaged by STM. In the following studies we address the local electronic structure via STM, but also gain additional insight into the electronic transport behavior in macroscopic magnetotransport experiments. $^{4}$

In section 4.2, we introduce doping with single nitrogen atoms in graphene. The content of this section is published as Ref. [198], "Short-range ordering of ion-implanted nitrogen atoms in SiC-graphene". In this structural analysis, we use STM topography measurements to investigate substitutional nitrogen implanted by low-

\footnotetext{
${ }^{4} \mathrm{An}$ in-depth introduction to the topic is given in section 4.1 .
} 
energy ion implantation. We find that only $10 \%$ of the ions get implanted, most likely due to adsorbate layers present during the ion bombardment. Moreover, we find that they are not randomly distributed, but observe a short-range ordering triggered by the $6 \times 6$-corrugation emerging from the underlying substrate.

In section 4.3, we investigate the influence of single boron, nitrogen and carbon atoms in graphene by STM, scanning tunneling spectroscopy (STS) and additionally their influence on transport. The content of this section is published as Ref. [200], "Doping of Graphene by Low-Energy Ion Beam Implantation: Structural, Electronic, and Transport Properties". We find by using STS that incorporation of nitrogen and boron atoms leads to effective doping of the graphene sheet and reduces or raises the position of the Fermi level. Additionally, the influence of foreign atoms and defects is investigated in macroscopic transport experiments. While for all samples the sheet resistance increases compared to pristine graphene, this effect is especially pronounced for samples with lattice defects (e.g. vacancies) and less for dopant atoms only. The positive magnetoresistance of pristine graphene changes to a strong negative one for ion-implanted samples by the effect of weak localization.



Figure 0.2: Substitutional nitrogen atom in SiC-graphene. $8 \mathrm{~nm} \times 8 \mathrm{~nm}$ constant current topography of SiC-graphene with a single nitrogen dopant implanted into the graphene lattice in the center of the image $\left(\mathrm{V}_{\text {Bias }}=-0.7 \mathrm{~V}, \mathrm{I}_{\mathrm{T}}=100 \mathrm{pA}\right)$. 
In chapter 1 and 2 an introduction to the theoretical background and the experimental methods is given, respectively. In chapter 5 we discuss the results of this thesis, put them into a broader context and give an outlook for future research.

The two result sections discussed above have been edited from their appearance in the respective journals. This concerns the numbering of figures, equations and tables as well as the font style. In the section "Darstellung der wissenschaftlichen Zusammenarbeit" on page 173 I state how the results of this thesis have been obtained and which parts have been conducted by myself or by coworkers and collaborators. 


\section{Table of Contents}

Introduction

Table of Contents vii

1 Theoretical background 1

1.1 Graphene 1

1.2 Electronic Transport in mesoscopic systems 6

1.2.1 Drude-Sommerfeld model and Boltzmann equation 6

1.2.2 Landauer Formula and Residual Resistivity Dipole 8

1.2.3 Evaluation of electronic transport in 2D 10

1.2.4 Magnetotransport 12

$\begin{array}{lll}\text { 1.2.5 Weak localization } & 15\end{array}$

2 Introduction to the experiments 19

2.1 Sample preparation 20

2.1.1 Epitaxial-grown graphene on SiC 20

2.1.2 CVD-grown graphene on $\mathrm{SiO}_{2} \quad 24$

2.1.3 Low-energy ion-implantation in graphene 24

2.2 Methods 25

2.2.1 Scanning Tunneling Microscopy 25

2.2.2 Scanning Tunneling Spectroscopy 27

2.2.3 Scanning Tunneling Potentiometry 28

2.2.4 Atomic Force Microscopy 34

$\begin{array}{lll}2.2 .5 & \text { Transport measurements } & 37\end{array}$

3 Transport properties of localized defects in graphene investigated by scanning probe methods

3.1 Introduction 39

3.2 Spatial extent of a Landauer residual-resistivity dipole in graphene quantified by scanning tunnelling potentiometry 
3.2.1 Introduction 43

3.2.2 Results 44

3.2.3 Discussion 48

3.2.4 Methods 50

3.2.5 Acknowledgement 51

3.2.6 Author contributions 51

3.2.7 Supplementary Information 52

3.3 Local transport measurements in graphene on $\mathrm{SiO}_{2}$ using Kelvin probe force microscopy $\quad 64$

3.3.1 Introduction 64

3.3.2 Results and discussion 65

$\begin{array}{lll}\text { 3.3.3 Supplementary Information } & 74\end{array}$

3.4 Magnetotransport on the nano scale 82

3.4.1 Introduction 82

3.4.2 Results 83

3.4.3 Discussion $\quad 89$

$\begin{array}{lll}3.4 .4 \text { Methods } & 91\end{array}$

The authors declare no competing financial interests. 92

3.4.5 Supplementary Information 93

$4 \begin{aligned} & \text { Substitutional doping in epitaxial graphene } \\ & \text { using low energy ion implantation }\end{aligned}$

4.1 Introduction 113

4.2 Short-range ordering of ion-implanted nitrogen atoms in SiCgraphene 116

4.2.1 Acknowledgement 124

4.2.2 Supplementary Information 124

4.3 Doping of Graphene by Low-Energy Ion Beam Implantation: Structural, Electronic, and Transport Properties 127

4.3.1 Author Contributions 136

$\begin{array}{lll}\text { 4.3.2 Acknowledgement } & 137\end{array}$

$\begin{array}{ll}\text { 4.3.3 Supporting Information } & 137\end{array}$

5 Discussion 142

5.1 Transport properties of extended defects 142

5.1.1 Defects in graphene on $\mathrm{SiO}_{2}$ 
5.1.2 Defects in SiC-graphene

5.1.3 Comparison to defects in other systems 146

5.2 Ion implantation of single dopants 149

$\begin{array}{lll}5.3 \text { Outlook } & 151\end{array}$

$\begin{array}{ll}\text { Bibliography } & 155\end{array}$

Darstellung der wissenschaftlichen Zusammenarbeit $\quad 173$

$\begin{array}{ll}\text { Danksagung } & 177\end{array}$

$\begin{array}{ll}\text { Curriculum Vitae } & 181\end{array}$ 



\section{Theoretical background}

This chapter provides the theoretical background for the physical processes discussed in this thesis. A brief introduction is given to the sample system graphene, where we discuss the peculiarities of this $2 \mathrm{D}$-material needed for the following chapters (section 1.1). Next, different concepts of transport are treated in section 1.2 starting from the Drude-Sommerfeld-model and the Boltzmann equation in subsection 1.2.1. The physics of a local scatterer in the framework of the Landauer formula and the Landauer residual resistivity dipole are discussed in subsection 1.2.2. Subsection 1.2 .3 is dealing with the evaluation of electronic transport in 2 dimensions. The influence of a magnetic field on transport is covered in subsection 1.2.4 and 1.2.5 discussing magnetotransport in general and weak localization, respectively.

\subsection{Graphene}

Graphene has been one of the most intensively investigated materials of the past decade due to its astonishing extraordinary properties. For the first realization in 2004[135] and subsequent characterization André Geim and Konstantin Novoselov received the Nobel Prize in 2010 [151]. Moreover, their discovery also triggered on the one hand studies in related 2D-Materials, e.g. boron nitride $(B N)$, tungsten $\left(\mathrm{WS}_{2}\right)$ and molybdenum diselenide $\left(\mathrm{MoS}_{2}\right)$.[55] On the other hand, other Dirac materials such as topological insulators with similar electronic properties were found subsequently.

While a variety of unusual and fascinating physics has been observed in graphene [56], within the framework of this thesis it can be mostly treated as a conventional 2D metal. This is due to the fact that first, graphene on silicon carbide ( $\mathrm{SiC})$ is heavily $n$-doped (See subsection 2.1.1). Second, several transport effects such as the half integer quantum Hall effect [215], Klein tunneling [86] or hydrodynamic electron flow [7] are either detected as small corrections in electronic transport or require special device fabrication to show their fingerprint. Therefore, in the following we only review the crystal structure of graphene and the bare essentials of the resulting electronic structure. A more detailed description of the electronic properties of graphene can be found in [129, 151, 180].

A single layer of sp2-hybridized carbon can be described as a triangular Bravais lattice with two atoms per unit cell 


$$
\boldsymbol{a}_{1}=\left(\frac{\sqrt{3} a}{2} \mid \frac{a}{2}\right), \quad \boldsymbol{a}_{2}=\left(\frac{\sqrt{3} a}{2} \mid-\frac{a}{2}\right)
$$

With the lattice constant $a=\left|a_{1}\right|=2.46 \AA$ [129]. This is depicted in Figure 1.1a. Figure $1.1 \mathrm{~b}$ shows the reciprocal lattice with the reciprocal lattice vectors

$$
b_{1}=\left(\frac{2 \pi}{\sqrt{3} a} \mid \frac{2 \pi}{a}\right), \quad b_{2}=\left(\frac{2 \pi}{\sqrt{3} a} \mid-\frac{2 \pi}{a}\right)
$$

as well as the Brillouin zone boundaries, that end at the K-points

$$
\boldsymbol{K}_{+}=\frac{4 \pi}{3 a}\left(\frac{\sqrt{3}}{2} \mid-\frac{1}{2}\right), \quad \boldsymbol{K}_{-}=\frac{4 \pi}{3 a}\left(\frac{\sqrt{3}}{2} \mid+\frac{1}{2}\right)
$$

(a)

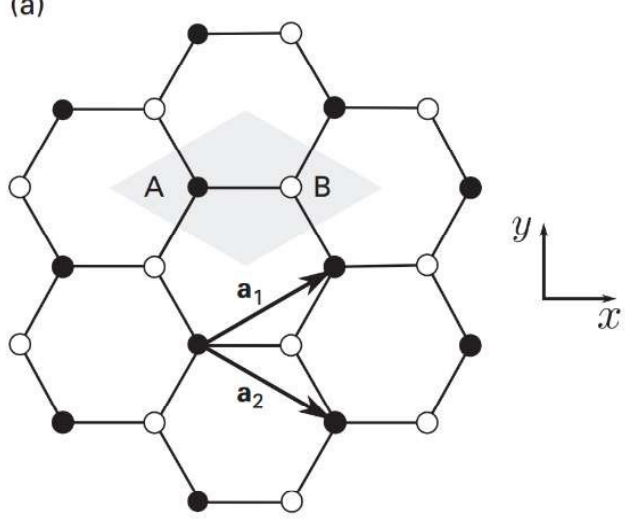

(b)

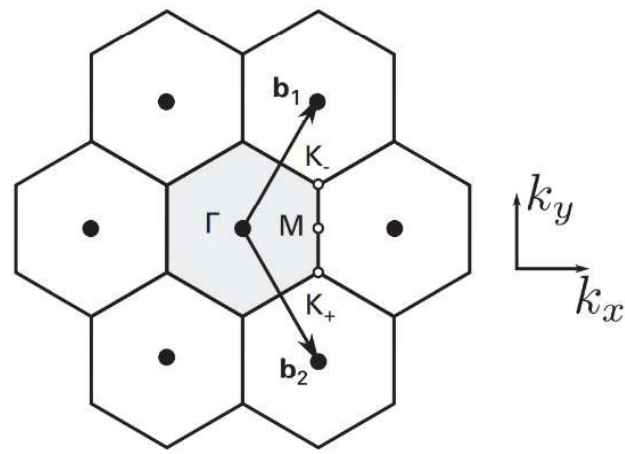

Figure 1.1: Bravais lattice of graphene. (a) Hexagonal Bravais lattice of graphene with lattice vectors $\boldsymbol{a}_{1} / \boldsymbol{a}_{2}$. Filled (empty) dots represent atoms belonging to sublattice A (B) of the two atom basis. (b) Reciprocal lattice with reciprocal lattice vectors $\boldsymbol{b}_{\mathbf{1}} / \boldsymbol{b}_{\mathbf{2}}$ and including borders of the Brillouin zone (grey shaded area) and high symmetry points. Reprinted (adapted) with permission from [180]. Copyright (2014) Cambridge University Press.

Using a tight-binding approximation, the electronic structure of graphene was already studied in 1947 by Wallace.[190] Considering a Hamiltonian with nearestneighbor hopping only and taking only $\mathrm{p}_{\mathrm{z}}$-orbitals into account, one finds the dispersion relation ${ }^{5}$

$$
\begin{gathered}
E(\boldsymbol{k})= \pm \gamma_{0} \sqrt{3+f(\boldsymbol{k})} \\
\text { with } f(\boldsymbol{k})=2 \cos \left(\sqrt{3} k_{y} a\right)+4 \cos \left(\frac{\sqrt{3}}{2} k_{y} a\right) \cos \left(\frac{3}{2} k_{x} a\right)
\end{gathered}
$$

by solving the time-independent Schrödinger-equation. Here, $\gamma_{0}$ is the hopping energy between first neighbor $\pi$-orbitals with values ranging from 2.9-3.1 eV.[23, 38]

\footnotetext{
${ }^{5}$ Including next nearest neighbor hopping adds a term $-\gamma_{0}{ }^{\prime} f(\boldsymbol{k})$ with $\gamma_{0}{ }^{\prime} \approx 0.1 \mathrm{eV}[33,129]$ and thus breaks the electron-hole symmetry.
} 
Moreover, the plus (minus) sign corresponds to the bonding $\pi$-band (antibondig $\pi^{*}$-band) and the function is symmetric around zero. Eq. (1.4) is plotted in Figure 1.2. Apparently, the only accessible states at the Fermi-level are located the Kpoints $\boldsymbol{k}=\boldsymbol{K}_{ \pm}$. The dispersion around the K-points results in six cones. Expanding in the low-energy limit around them $\boldsymbol{k}=\boldsymbol{K}_{ \pm}+\delta \boldsymbol{k}$ yields the linear dispersion for graphene

$$
E(\delta \boldsymbol{k})= \pm \hbar v_{F}|\delta \boldsymbol{k}|
$$

Here, the Fermi velocity is given by

$$
v_{F}=\frac{\sqrt{3} \gamma_{0} a}{2 \hbar} \approx 1 \cdot 10^{6} \frac{\mathrm{m}}{\mathrm{s}}
$$

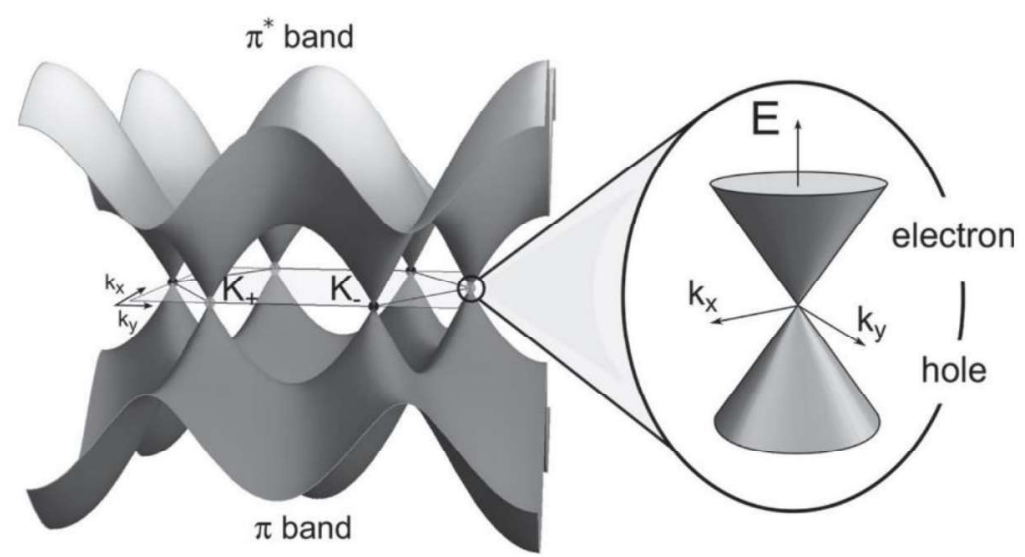

Figure 1.2: Band structure of graphene. For the tight-binding model described in the text, the band structure consists of symmetric $\pi$ - and $\pi^{*}$-bands. Here, the Fermi level is located at the $\boldsymbol{K}_{ \pm}$points. Inset: Linear dispersion around the Dirac cone. Reprinted (adapted) with permission from [180]. Copyright (2014) Cambridge University Press.

using the values for $t$ and $a$ stated above. Replacing $v_{F} \rightarrow c$ yields the energy relation of a massless 2D Dirac Fermion $\lim _{m \rightarrow 0} \pm c \sqrt{p^{2}+m^{2} c^{2}}$. Therefore, in the lowenergy limit, electrons in graphene can be treated as slow, relativistic particles using the Weyl-Dirac- instead of the Schrödinger-equation.

Moreover, the two different sublattices $\boldsymbol{K}_{ \pm}$are electronically not equal. This can be seen in the wavefunctions around the K-points related to the solution of Eq. (1.5)

$$
\Psi_{ \pm, \mathbf{K}_{+}}(\delta \mathbf{k})=\frac{1}{\sqrt{2}}\left(\begin{array}{c}
\mathrm{e}^{-\mathrm{i} \theta_{\delta \mathbf{k}} / 2} \\
\pm \mathrm{e}^{\mathrm{i} \theta_{\delta \mathbf{k}} / 2}
\end{array}\right) \quad \Psi_{ \pm, \mathbf{K}_{-}}(\delta \mathbf{k})=\frac{1}{\sqrt{2}}\left(\begin{array}{c}
\mathrm{e}^{\mathrm{i} \theta_{\delta \mathbf{k}} / 2} \\
\pm \mathrm{e}^{-\mathrm{i} \theta_{\delta \mathbf{k}} / 2}
\end{array}\right)
$$

6 The Fermionic character arises here from the pseudo-spin discussed below. 
Here, again the \pm sign belongs to the solutions of the two $\pi$-bands. $\theta_{\delta \mathbf{k}}$ is defined as

$$
\theta_{\delta \mathbf{k}}=\tan ^{-1}\left(\delta \mathbf{k}_{x} / \delta \mathbf{k}_{y}\right)
$$

The two sublattices $\boldsymbol{K}_{ \pm}$are connected by time-reversal symmetry ${ }^{7}$ and it can be shown that the wavefunction forms a two-component spinor.[129] A new quantum number, the helicity or chirality, is defined as the projection of the momentum operator along the Pauli-spin matrix $\boldsymbol{\sigma}=\left(\sigma_{x}, \sigma_{y}\right)$

$$
\hat{h}=\frac{1}{2} \boldsymbol{\sigma} \cdot \frac{\boldsymbol{p}}{|\boldsymbol{p}|}
$$

This (pseudo)-spin is a consequence of the inequality of the two sublattices $\boldsymbol{K}_{ \pm}$ (The sublattice degree of freedom [180]) and is not related to the actual electron spin. Another property arising from the wavefunctions in Eq. (1.7) is a Berry's phase of $\pi,{ }^{8}$ characteristic for spinors: Changing $\theta_{\delta \mathbf{k}}$ by $2 \pi$ leads to a change in sign for the functions corresponding to a phase change of $\pi$. This just changes the wavefunction to the one of the other sublattice. Figure 1.3 elucidates the physics involving Berry's phase and the pseudo-spin.[129]

The presence of the pseudo-spin leads to interesting phenomena such as Klein Tunneling [86] and the absence of backscattering [129]. It also leads to deviations from the conventional weak localization theory [123] as discussed in subsection 1.2.5.

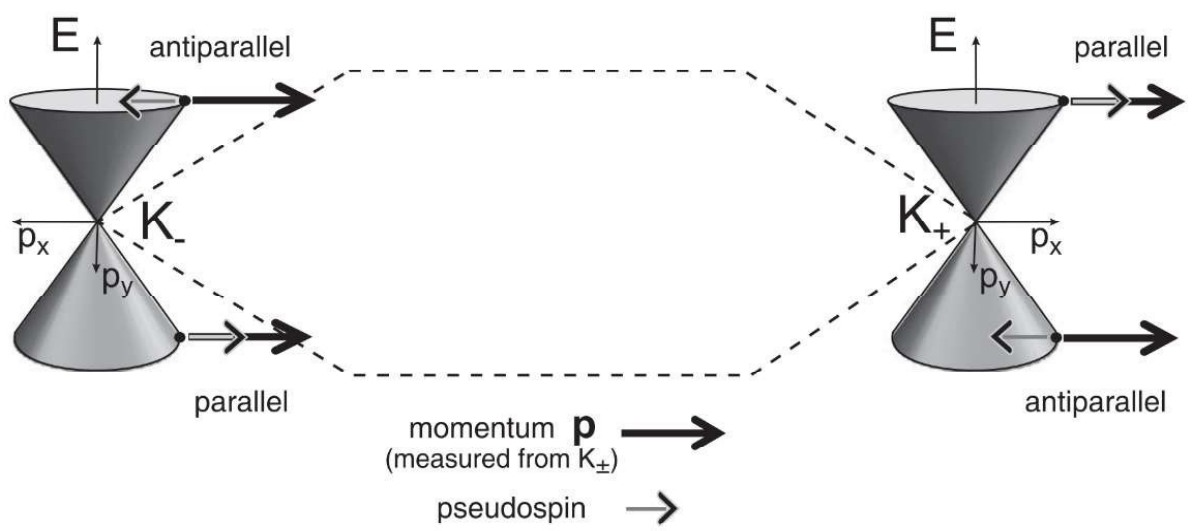

Figure 1.3: Pseudo-spin in graphene. Momentum $p$ and pseudo-spin sketched for the two inequivalent sublattices $\boldsymbol{K}_{ \pm}$. Reprinted (adapted) with permission from [180]. Copyright (2014) Cambridge University Press.

\footnotetext{
${ }^{7}$ By putting the origin to the M-point of the Brillouin-zone, time reversal becomes equivalent to a mirror projection along $\mathbf{k}_{\mathrm{x}}$.

8 In a formal description, the Berry phase is defined as a gauge-invariant quantity $\gamma=$ $i \oint\left\langle\left\langle\psi_{k}\left|\nabla_{k}\right| \psi_{k}\right\rangle d \boldsymbol{k}\right.$, The total phase accumulated via a closed-loop rotation in k-space. This exactly yields $\pi$ for the wave functions in (1.17). An in-depth discussion can be found in Ref. [180].
} 
Within the scope of this thesis the transport properties of transition regions between monolayer and bilayer graphene (MLG/BLG) are investigated. Bilayer graphene consists of two stacked layers of graphene on top of each other. The electronic properties of bilayer graphene depend crucially on the stacking [111]. For graphene on $\mathrm{SiC}(0001) \mathrm{AB}$-stacking (Bernal-stacking) is found [100] which is sketched in Figure 1.4a. The additional superposition of $\mathrm{p}_{z}$-orbitals at the positions $\mathrm{B} 1$ and $\mathrm{A} 2$ lead to additional bands. This can be derived in a tight-binding approach analog to Eq. (1.4) with additional hopping parameters $\gamma_{1}$ introducing the interlayer coupling [42, 151]. The result is shown in Figure 1.4b. In contrast to MLG, bilayer bands show a parabolic dispersion. Moreover, a band gap opens for different energies of the upper and lower layer. This can be induced either due to a different
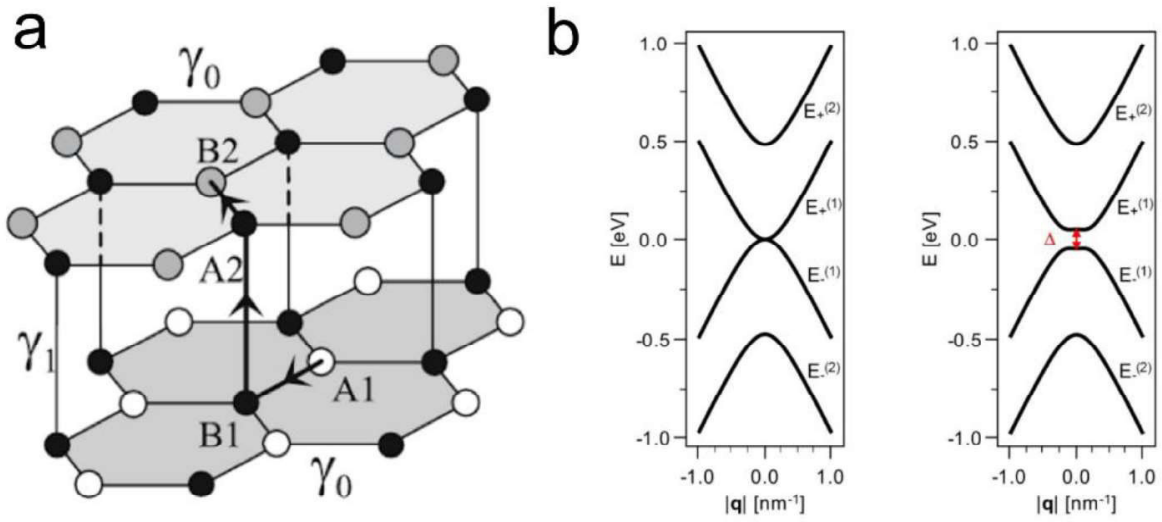

doping or an applied externa field. For bilayer graphene on $\mathrm{SiC}(0001)$ this is indeed the case due to the strong substrate induced $n$-doping (See subsection 2.1.1).

Figure 1.4: Electronic structure of bilayer graphene. (a) Side view of the geometry of bilayer graphene with $A B$-stacking. The different sublattice atoms are labeled $A$ and $B$ followed by numbers indicating the layer they are in. The hopping parameter $\gamma_{0}$ mediates intralayer coupling while $\gamma_{1}$ is used to introduce interlayer coupling. Taken from Ref. [151]. (b) Tight-binding calculation of the dispersion relation at the K-point for the bilayer system shown in (a). In contrast to MLG two bands are present and the dispersion has now become parabolic. For the diagram on the right a potential difference $\Delta=0.1 \mathrm{eV}$ between the two layers leads to a band gap opening while on the left both layers are equally (Parameters: $\gamma_{1}=0.48 \mathrm{eV}, \mathrm{v}_{\mathrm{F}}=1.05 \cdot 10^{6} \mathrm{~m} / \mathrm{s}$ ). Taken from Ref. [42].

Due to the change in electronic structure between MLG and BLG, wave functions at the interface needs to be matched. The resulting finite transmission at the boundary [126] is giving rise to a change in conductivity in the framework of the Landauer formalism (See subsection 1.2.2). The behavior of the resistance of this interface is studied in chapter 3. 


\subsection{Electronic Transport in mesoscopic systems}

To study electron transport from macroscopic sample dimensions down to the scale of a few Ångströms different concepts of transport have to be used. Therefore, this section gives an introduction to different theories that are able to describe different aspects of transport phenomena, starting with the rather general DrudeSommer model towards a theory of weak localization in graphene.

The model of Drude for electron transport with the extensions made by Sommerfeld and Boltzmann introduced in subsection 1.2.1 is used in this thesis to describe and understand the scattering processes in pristine graphene regions (In contrast to local defects) in chapter 3 and section 4.3. Here, the sheet resistance is influenced by delocalized scattering contributions such as electron-phonon- and electron-electron-scattering. The implantation of substitutional doping atoms is also influencing the conductivity and the charge carrier concentration of the sample treated in section 4.3 of chapter 4 . Both is captured in the framework of this models.

In contrast, the localized voltage drop at atomic-scale defects such as steps and interfaces using STP/KPFM in chapter 3 is described in the framework of the Landauer-formalism in subsection 1.2.2. Additionally, subsection 1.2.3 comments on how both localized scatterers and sheet resistances are evaluated from STP/KPFM measurements in chapter 3.

Moreover, magnetic field dependent measurements have been conducted in this thesis. How the sample geometry alters the observed magnetic field dependence of the sample resistance (magnetoresistance) is treated in subsection 1.2.4. This is crucial for the choice of the sample geometry in section 3.4 of chapter 3 . For low temperatures and in the presence of defects the effect of weak localization described in subsection 1.2 .5 can be studied by magnetoresistance measurements. This is done for ion implanted samples treated in section 4.3 of chapter 4 .

\subsubsection{Drude-Sommerfeld model and Boltzmann equation}

One of the first and simplest semiclassical descriptions of electron transport in a metal was developed by Paul Drude in 1900 [39, 40]. It treats the electrons within the kinetic gas theory.[77] The conductor is described as an ionic crystal in which the electrons move freely as classical particles and gain momentum $\mathbf{k}$ from the external electric field $\mathbf{E}$.

$$
\hbar \dot{\mathbf{k}}=m \dot{\mathbf{v}}=-e \mathbf{E}
$$

For a steady state current density $j=-e n \mathrm{v}_{\mathrm{D}}$, the velocity $\mathbf{v}$ of each electron can be replaced with the average drift velocity $v_{D}$. The scattering of electrons with the 
ionic crystal lattice is characterized by the momentum relaxation time $\tau$ (average scattering time) treated as a phenomenological friction term leading to

$$
m \dot{v}_{D}+\frac{m}{\tau} v_{D}=-e E
$$

For stationary conditions $\dot{\mathrm{v}}_{D}=0$ Ohm's law in the framework of the Drude theory can be written as

$$
j=-e n \mathrm{v}_{\mathrm{D}}=\frac{n e^{2} \tau}{m} \cdot E=\sigma \cdot E \quad \text { with } \quad \sigma=\frac{n e^{2} \tau}{m}
$$

This gives an intuitive picture of the local conductivity in a metal that is here proportional to both the time scale $\tau$ before momentum relaxation takes place as well as the total density of electrons $n$ available. Because the latter is a rather obvious influence, the electron mobility $\mu=\sigma / n e=e \tau / m$ is additionally defined.

Still, the Drude theory represents only a crude approximation, since it neglects the Pauli principle and the fact that electrons do not get scattered at positive charged lattice atoms. It moreover includes all conduction electrons in current transport, instead of obeying the Fermi-Dirac statistics.[60, 77] The latter has been accounted for by the extension from Arnold Sommerfeld by deriving the change in momentum from a distorted Fermi surface (Figure 1.5).


Figure 1.5: Fermi-function in Drude-Sommerfeld model. (a) Fermi-surface without electric field applied. The dots symbolize allowed wave vectors in k-space. (b) Displacement of the Fermi surface under the influence of an electric field shifting the electron distribution by a fraction $\delta \boldsymbol{k}=-e \boldsymbol{E} \delta t / \hbar$. Adapted from Ref. [77].

A more elaborate approach, the Boltzmann equation, compensates for all of these problems. Following the idea of Sommerfeld, one can derive for a homogeneous medium the following linearized Boltzmann-equation of electron transport for the distribution function $f(\boldsymbol{k})$ and the electric field $\boldsymbol{E}$ [77] 


$$
f(\boldsymbol{k}) \approx f_{0}(\boldsymbol{k})+\frac{e \tau(\boldsymbol{k})}{\hbar} \boldsymbol{E} \cdot \nabla_{k} f_{0}(\boldsymbol{k})
$$

Here, $f_{0}(\boldsymbol{k})$ is the distribution in equilibrium and the momentum-dependent relaxation time $\tau(\boldsymbol{k})$. Calculating now the current density $j$ by integration over momentum space yields

$$
\sigma=\frac{n e^{2} \tau\left(E_{F}\right)}{m^{*}}
$$

Here, the effective mass $m^{*}$ accounts for the band structure of the material and $\tau\left(E_{F}\right)$ indicates that only electrons close to the Fermi surface can contribute to the current transport. This can be seen from equation (1.13), since for integration over $\boldsymbol{k} \nabla_{k} f_{0}(\boldsymbol{k})$ only gives non-vanishing contributions close to the Fermi-level $E_{F}$.

Within the extended Drude-Sommerfeld model, electronic transport can be quite easily modeled by a classical resistor network model [72]. This only accounts for electrostatic changes by dividing the (local or macroscopic) sample region into a finite number of knots. Each knot is assigned a resistance, depending on structural features (pristine sheet, defect, etc.). By comparison with the experimental data (STP/KPFM) sheet resistances and defect resistances can be evaluated. Resistornetwork models have been used in section 3.2 and 3.3. In chapter 3.4 a similar finite-element-method has been applied to implement an additional magnetic field. An in-depth description can be found in subsections 3.2.7.6, 3.4.5.3, 3.3.3.1 and 3.3.3.3 as well as Ref. [42, 72].

\subsubsection{Landauer Formula and Residual Resistivity Dipole}

In the focus of this thesis is the analysis of local voltage drops in the vicinity of defects by the method of STP. While the Drude model treats defects as an average scattering time only, a description of electron transport close to a specific defect has been developed by Rolf Landauer in 1957 [99] and is often referred to as the Landauer residual resistivity dipole [31]. It assumes that the defect has a finite transmission $T$ leading to a readjustment of the electrochemical potential (ECP) $\mu_{\mathrm{ECP}}$ in its vicinity.

For a perfect ballistic conductor with electrochemical potentials $\mu_{\mathrm{ECP}, 1}$ and $\mu_{\mathrm{ECP}, 2}$ at its contacts (see Figure 1.6a), the current-voltage characteristic can be written as [31]

$$
I=G \cdot \frac{\mu_{\mathrm{ECP}, 1}-\mu_{\mathrm{ECP}, 2}}{e}=\frac{2 e^{2}}{h} M \cdot \frac{\mu_{\mathrm{ECP}, 1}-\mu_{\mathrm{ECP}, 2}}{e}
$$

where $M$ is the number of modes present in the conductor and $G$ the conductivity of the conductor. Since it is assumed to be ballistic, no voltage drops inside the sample, but only at the contacts due to the mismatch in number of modes.[31] 
Deviations from this ideal configuration are now described in the Landauer formula. Introducing a transmission probability $T<1$ of a local scatterer, we can generalize

$$
G=\frac{2 e^{2}}{h} M T \approx \frac{2 e^{2}}{h} \cdot \frac{k_{F}}{\pi} W \cdot T
$$

where the number of modes has been estimated by $M \approx W k_{F} / \pi$ with $W$ being the width of the conductor. A certain fraction $1-T$ of incident electrons gets reflected at the defect while the fraction $T$ is transmitted. The situation is sketched in Figure 1.6a. The reflection of electrons changes the population of the distribution function $f^{+}\left(E, \mu_{\mathrm{ECP}, 1}\right)$ for forward scattering states $k^{+}$as well as of $f^{-}\left(E, \mu_{\mathrm{ECP}, 2}\right)$ for backwards scattering states $k^{-}$. In the close vicinity of the scatterer this leads to a change in the ECP $\mu_{\mathrm{ECP}, 1} / \mu_{\mathrm{ECP}, 2}$ so that the voltage

$$
e V=(1-T)\left[\mu_{\mathrm{ECP}, 1}-\mu_{\mathrm{ECP}, 2}\right]
$$

drops across the scatterer (Figure 1.6b). This leads to an ECP

$$
\mu_{\mathrm{ECP}}(x)=\mu(x)-e \phi(x)
$$

which is changing abruptly across the defect (Figure 1.6b), since it is governed by the change in transmission. Simultaneously the backscattering leads to an increase (decrease) of charge carriers concentration $n(x)$ in front of (behind) the scatterer and thus to a change in chemical potential $\mu(x)$ (Figure 1.6d). For this emerging dipole (Landauer residual resistivity dipole) we can employ the Poisson equation and find the change in electrostatic potential $\phi(x)$. In contrast to the ECP, the conduction band edge $E_{s}$ [which follows the electrostatic potential $\phi(x)$ ] cannot follow such a sharp transition, but changes smoothly over a screening length $l$ (Figure 1.6c).[31]

Based on Eq. (1.16), the conductance across the defect can be written as [122]

$$
\sigma_{\text {Defect }}=\frac{2 e^{2}}{h} \cdot \frac{k_{F}}{\pi} \cdot T=\rho_{\text {Defect }}^{-1}
$$

The latter $\rho_{\text {Defect }}[\Omega \mathrm{m}]$ is the defect resistance, which we will use in the following chapters to characterize the scattering at localized defects in graphene.

The spatial dependence $x$ of Eq. (1.18) in the argument of $\mu_{E C P}(x)$ indicates a subtle, but important point. Usually functions of state (as the ECP) are only welldefined in equilibrium. This can be avoided by defining a local ECP established by the local interplay of energy relaxation and changes in the distribution functions with the boundary condition of conservation of number of electrons. A detailed discussion of this problem can be found in Ref. [31, 72]. 


\subsubsection{Evaluation of electronic transport in 2D}

To characterize the scattering mechanisms in our sample system, we can locally evaluate the sheet resistance/conductivity and the defect resistance given in Eq. (1.14) and (1.19), respectively. Since graphene is a $2 D$ conductor, the definitions and dimensions of these quantities deviate from the better known 3D case.

The macroscopic sheet resistance is given by

$$
\rho_{\square, \text { macro }}=R \cdot \frac{W}{L} \quad[\Omega / \square]
$$
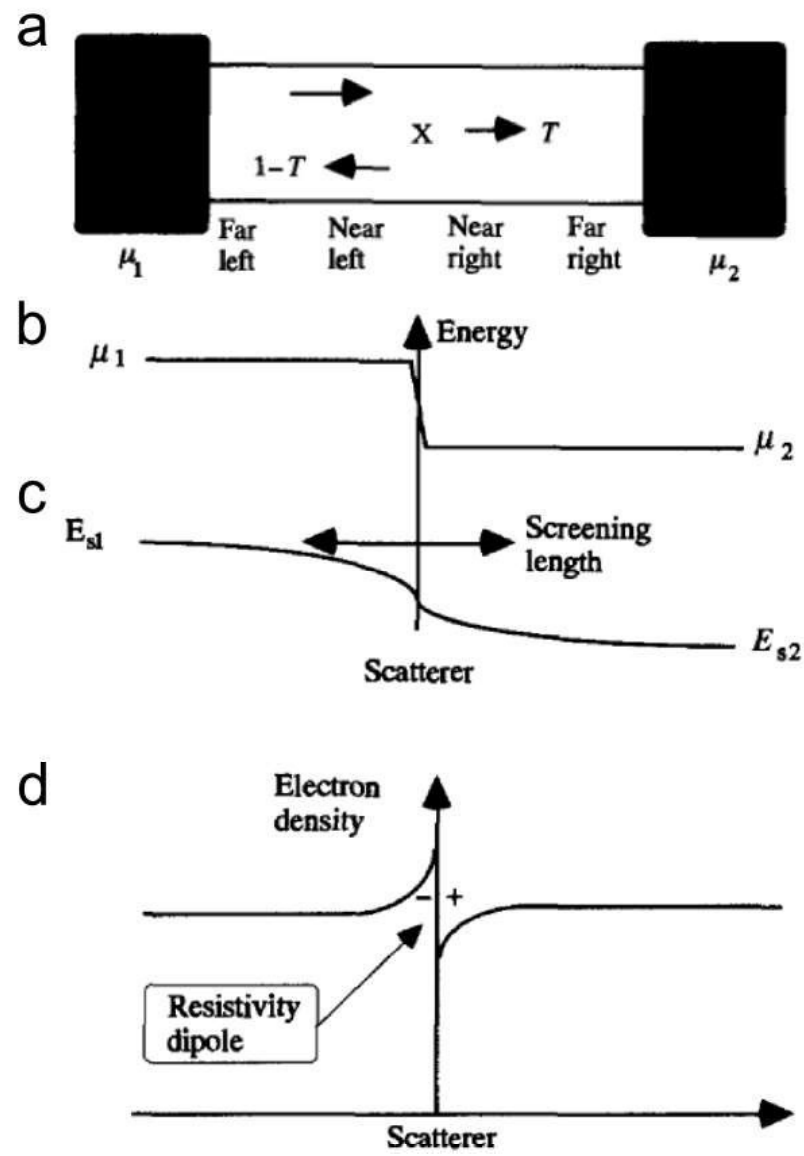

Figure 1.6: Landauer residual resistivity dipole at a local scatterer. (a) Ballistic conductor with leads $\mu_{1}$ and $\mu_{2}$ including a scatterer $(X)$ with a finite transmission $T$. (b) Electrochemical potential $\mu_{\mathrm{ECP}}(x)$, (c) conduction band edge $E_{s}(x)$ and (d) electron density $n(x)$ as a function of position $x$ around the scatterer. Reprinted (adapted) with permission from Ref. [31]. Copyright (1997) Cambridge University Press.

with the width $W$ and length $L$ as well as the total resistance $R$ of the sample (sample geometry shown in Figure 1.7a). The latter is defined as $R=V / I$ with the voltage difference between the two contact $V=\mu_{\mathrm{ECP}, 2}-\mu_{\mathrm{ECP}, 1}$ and the total electric current $I$. One of the major goals of this thesis is now to evaluate the electronic 
transport locally. In this case, we ask for the fraction $\Delta V$ that drops across the distance $\Delta L$ in the sample. Here, $\Delta L$ can be in the order of nanometers. Then we can write Eq. (1.20) as

$$
\rho_{\square, l o c a l}=\frac{\Delta V}{I} \cdot \frac{W}{\Delta L}=\frac{\Delta V}{\Delta L} / \frac{I}{W}=E_{\text {local }} / j \quad[\Omega / \square]
$$

where we introduced the local electric field $E_{\text {local }}=\Delta V / \Delta L$ and the current density $j=I / W$. For a perfectly homogeneous system as shown in Figure $1.7 \mathrm{~b}$, this again

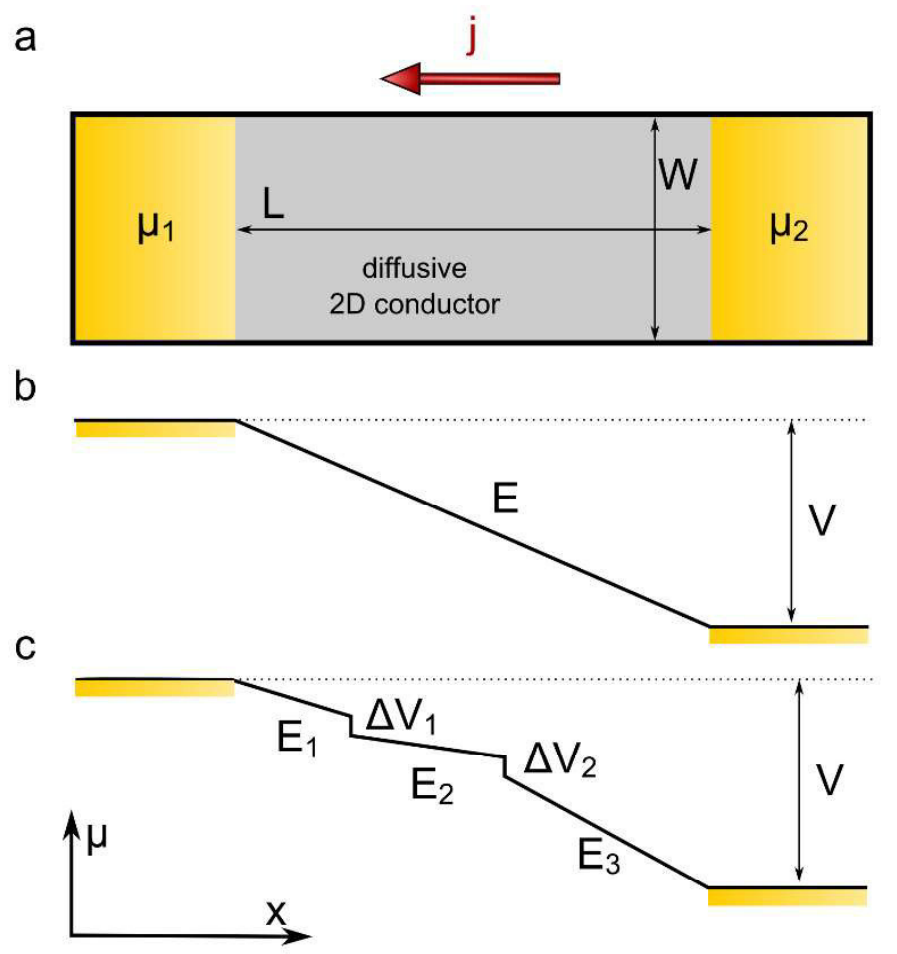

Figure 1.7: Electronic transport in 2D. (a) Diffusive 2D conductor of length $L$ and width $W$ with leads $\mu_{1}$ and $\mu_{2}$. (b) Voltage drop across the sample for a completely homogeneous system indicated by a homogeneous electric field $E$. (c) Voltage drop for different local electric fields $E_{i}$ as well as additional localized voltage drops $\Delta V_{i}$.

yields the macroscopic value $\rho_{\square, \text { local }}=\rho_{\square, \text { macro }}$. However, in the presence of local variations in sheet resistance or of local defects (Figure 1.7c) the local electric field $E_{\text {local }}$ deviates from the macroscopic one. This can be nicely seen by again writing Eq. (1.21) as

$$
\rho_{\square, \text { local }}=E_{\text {local }} / \frac{I}{W}=E_{\text {local }} \cdot W \cdot \frac{R}{V}=E_{\text {local }} \cdot L \cdot \frac{\rho_{\square, \text { macro }}}{V}=\frac{E_{\text {local }}}{E_{\text {macro }}} \cdot \rho_{\square, \text { macro }}
$$

where we defined $E_{\text {macro }}=V / L$. Thus, the local resistivity is expressed as its macroscopic counterpart multiplied by the ratio of electric fields.

Analogously, localized defects can be characterized by: 


$$
\rho_{\text {defect }}=\Delta V / j=\frac{\Delta V}{V} \cdot L \cdot \rho_{\square, \text { macro }}[\Omega \mathrm{m}]
$$

The change in dimension arises here from their definition to be a 1D interface in a 2D conductor.

For the simple case of a system translational invariant in y-direction ${ }^{9}$, the macroscopic sheet resistance can be written as the sum of the local parts by Kirchhoff's rule

$$
\rho_{\square, \text { macro }}=\sum_{i} x^{i} \cdot \rho_{\square, \text { local }}^{i}+\sum_{i} n^{i} \cdot \rho_{\mathrm{defect}}^{i}
$$

With the fraction $x^{i}=\Delta L^{i} / L$ of each different local sheet resistance as well as the defect concentration $n^{i}=N^{i} / L$ with the total number of defects $N^{i}$ of each type. ${ }^{10}$ One can undoubtedly argue that $\rho_{\square, \text { macro }}$ is not representing a sheet resistance, since it now contains different contributions from localized defects as well as locally varying sheet resistances. Nevertheless, this is the situation in (most) large scale transport experiments. It motivates the use of local transport studies by scanning tunneling potentiometry and other techniques to dissect the different contributions in Eq. (1.24).

\subsubsection{Magnetotransport}

With the resistivity $\rho=1 / \sigma$ of a $2 \mathrm{D}$ system, we define the magnetoresistance (MR) $\Delta \rho$, the change in resistance due to a magnetic field $\mathrm{B}$.

$$
\Delta \rho:=\frac{\rho(B)-\rho(0)}{\rho(0)}
$$

For a transverse magnetic field B, perpendicular to the current flow, charge carriers get deflected by the Lorentz-force. Generalizing the Drude conductivity introduced in Eq. (1.10) with an additional magnetic field $\boldsymbol{B}=(0,0, B)$ leads to

$$
\frac{m}{\tau} \boldsymbol{v}_{\boldsymbol{D}}=-e\left[\boldsymbol{E}+\boldsymbol{v}_{\boldsymbol{D}} \times \boldsymbol{B}\right]
$$

Solving for Ohm's law in the form $\boldsymbol{E}=\hat{\sigma}^{-1} \boldsymbol{j}$ in the limit of 2D transport yields [31]

\footnotetext{
${ }^{9}$ Additionally, the current density $j(\mathrm{x}, \mathrm{y})$ is also not necessarily constant, if the system is not translational invariant in $y$-direction as it is the case in Figure 1.7. This is discussed in detail in 3.3.3.1 of chapter 3 for the case of inhomogeneities caused by graphene wrinkles in graphene on $\mathrm{SiO}_{2}$ and additionally in Ref. [42] for steps and interfaces in SiC-graphene.

${ }^{10}$ We here neglect in this purely classical treatment phase-coherent effects such as weak localization treated in subsection 1.2.5.
} 


$$
\left(\begin{array}{l}
E_{x} \\
E_{y}
\end{array}\right)=\left[\begin{array}{cc}
m / e \tau & -B \\
B & m / e \tau
\end{array}\right]\left(\begin{array}{l}
v_{x} \\
v_{y}
\end{array}\right)=\sigma_{0}^{-1}\left[\begin{array}{cc}
1 & \mu B \\
-\mu B & 1
\end{array}\right]\left(\begin{array}{l}
j_{x} \\
j_{y}
\end{array}\right)=\left[\begin{array}{cc}
\rho_{0} & -R_{H} B \\
R_{H} B & \rho_{0}
\end{array}\right]\left(\begin{array}{l}
j_{x} \\
j_{y}
\end{array}\right)
$$

where we made use of the relations in Eq. (1.12). Moreover, the hall resistance $R_{H}=-1 /$ en was introduced. The subscript for $\sigma_{0}=1 / \rho_{0}$ indicates the zero-field conductivity/resistivity. Inverting the resistivity matrix yields [77]

$$
\hat{\sigma}=\frac{\sigma_{0}}{1+(\mu B)^{2}}\left[\begin{array}{cc}
1 & -\mu B \\
\mu B & 1
\end{array}\right]
$$

The magnetic field $B$ increases the off-diagonal elements.

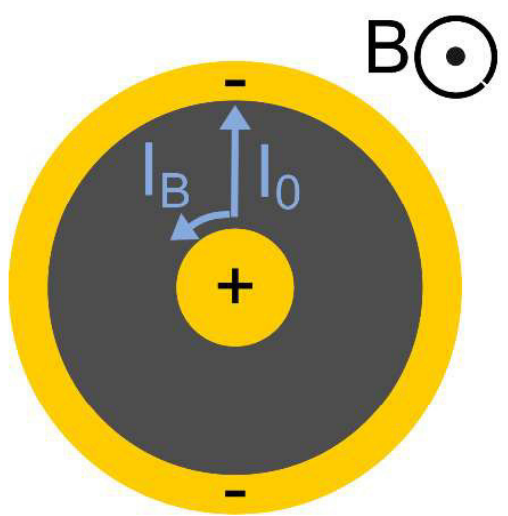

Figure 1.8: Corbino disk contact geometry. Contacts are given by an inner and an outer circle while a transverse (perpendicular) magnetic field $B$ is applied. For increasing $B$ the current $I_{0}$ changes by the additional component $\mathrm{I}_{\mathrm{B}}$. Thus, the path an electron has to travel in the medium with resistivity $\rho_{0}$ increases leading to a positive MR.
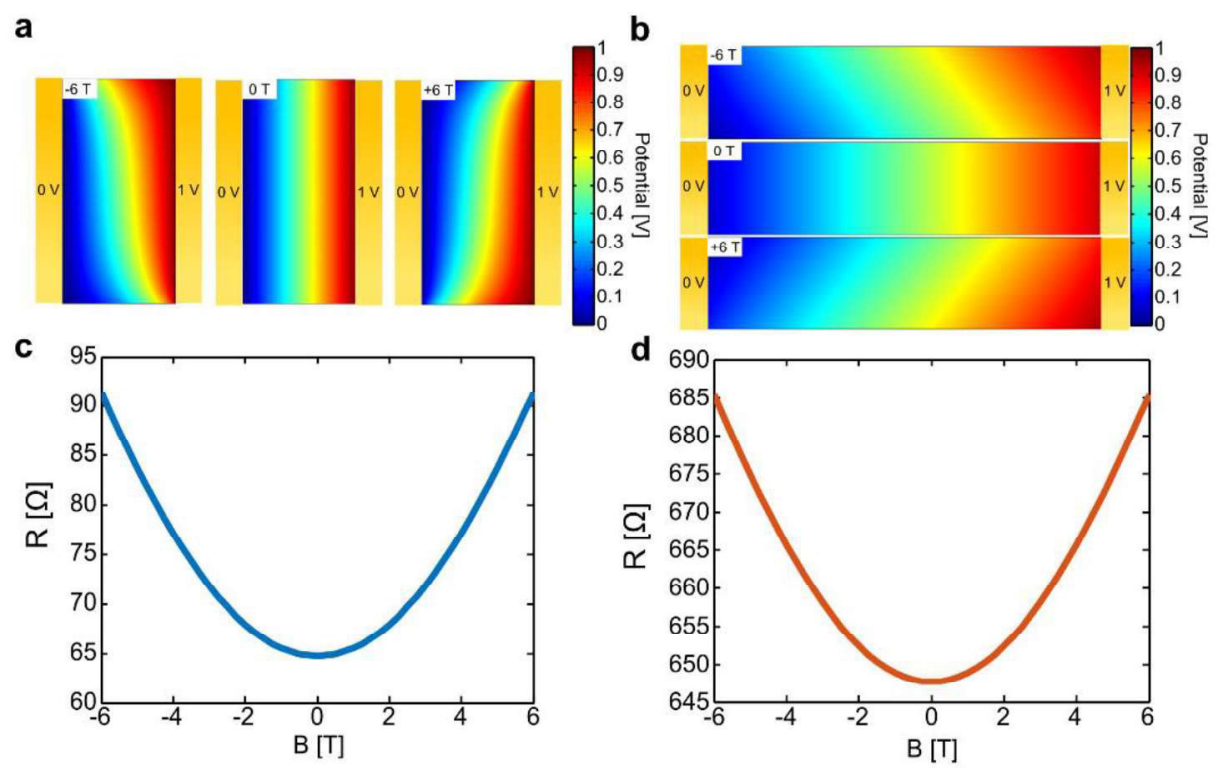

Figure 1.9: Potential distribution and Magnetoresistance for different contact geometries. (a) Sample in short-channel geometry with low ratio $L / W=1 \mathrm{~mm} / 2 \mathrm{~mm}=0.5$. (b) Long sample (Hall-geometry) with high ratio $L / W=10 \mathrm{~mm} / 2 \mathrm{~mm}=5$. (c) $R(B)$ for the 
sample geometry in a. (d) $R(B)$ for the sample geometry in c. (Simulation parameters: $\sigma_{0}=$ $7.84 \mathrm{mS} / \mathrm{n}=1.23 \cdot 10^{13} \mathrm{~cm}^{-2}$ )

The influence on the MR can best be understood in the geometry of a Corbino disk [32] (Figure 1.8). For no magnetic field, the electrons can directly flow from the inner to the outer contact, while for finite field they get deflected and have to spend a longer time in the sample. Therefore, the $\rho$ (B) increases. Since for this geometry the electric field does not change under the influence of a magnetic field, the change in MR can directly be seen from the conductivity tensor in Eq. (1.28) to scale with $\Delta \rho \propto(\mu \mathrm{B})^{2}$. This holds in general for other geometries. However, the effect can be more or less pronounced depending on the sample geometry.

Figure 1.9 shows finite element method simulations using COMSOL Multiphysics for different sample geometries in case of no magnetic field as well as for $\pm 6 \mathrm{~T}$. As can be seen in spatially resolved potential images in Figure 1.9a, the contacts induce fixed boundary conditions, since they are on one potential and thus the equipotential lines in their vicinity are heavily bent for applied magnetic field $B$. These regions are responsible for the MR. Therefore, short-channel samples with $L<W$ (Figure 1.9a) are dominated by the contact regions and show a strong MR (Figure $1.9 \mathrm{c}$, relative increase $\approx 50 \%)$. In contrast, for long thin geometries with $L>W$ shown in Figure 1.9b (Hall-geometry) this contribution vanishes, since a constant electric field gradient $E_{y}$ in $y$-direction has been established here. The respective MR-curve is shown in Figure $1.9 \mathrm{~d}$ with almost no dependence on magnetic field (obey different $y$-scale, relative increase $\approx 5 \%$ ). Figure 1.10 quantifies this observation demonstrating that the magnitude of the observed MR is especially present for short samples and is vanishing in the limit of perfect Hall geometries.

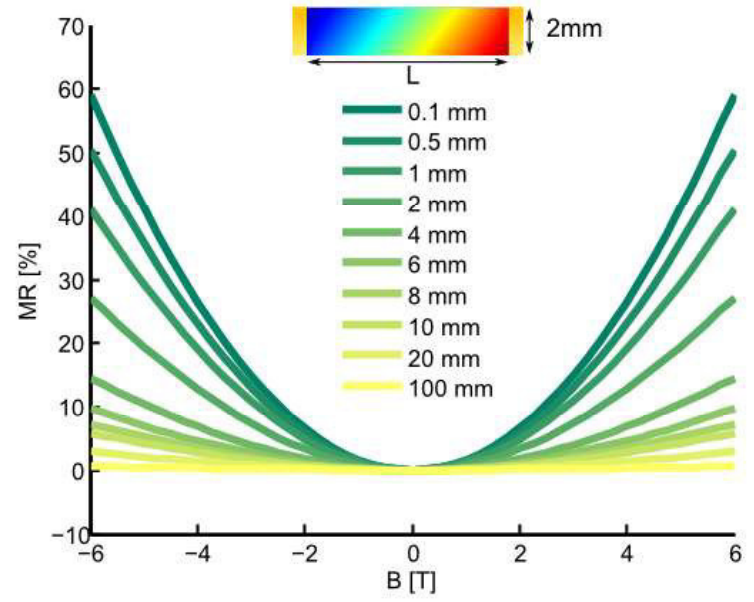

Figure 1.10: Magnetoresistance for different aspect ratios. Color-coded plots are simulated for different length $L$ of the sample while the width $W=2 \mathrm{~mm}$ is held constant. Thus, the aspect ratio $L / W$ changes. Accordingly, for increasing aspect ratio the MR decreases. (Simulation parameters as in Figure 1.9) 
In section 3.4 of chapter 3 the geometry was on purpose chosen to be short-channeled (Figure 1.9a) instead of using a Hall-geometry (Figure 1.9b). In this way deviations from the quadratic behavior are easier to detect due to the larger absolute change in MR.

\subsubsection{Weak localization}

Weak localization and anti-localization are phase-coherent transport phenomena that are directly linked to the presence of scattering centers in the conductor. Since both the phase coherence time $\tau_{\phi}$ and length $L_{\phi}$ are decreasing with increasing temperature, it is a low-temperature effect. Figure 1.11 depicts a random distribution of scatterers.

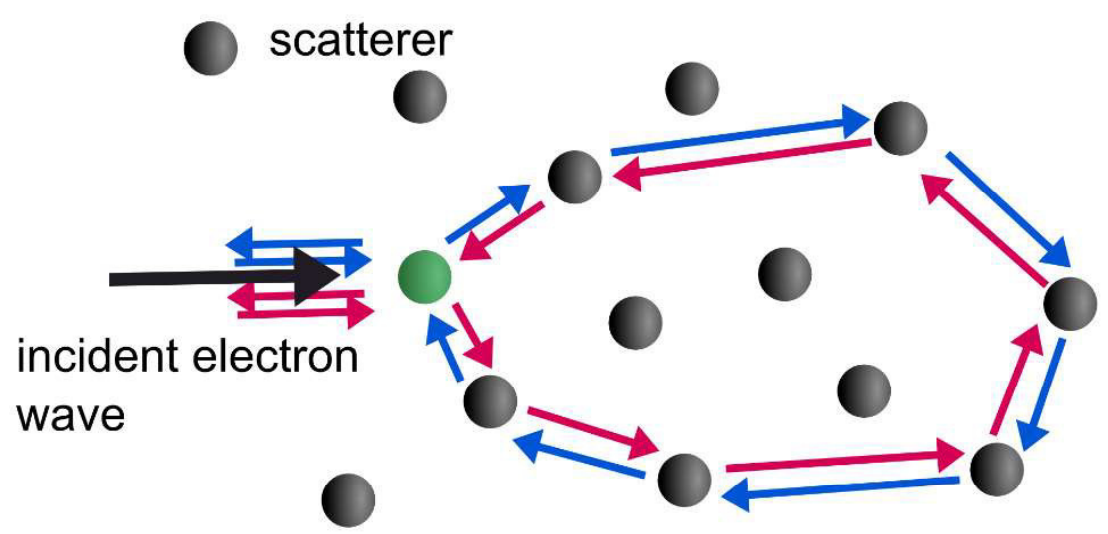

Figure 1.11: Schematic for weak localization. For a closed trajectory of scattering centers an incident electron wave (black) can possibly scatter clockwise (blue) and counterclockwise (red). Adapted from Ref. [180].

An incident electron wave can now scatter (amongst many possible trajectories) clockwise and counter-clockwise along the blue and red trajectory, respectively. If time-reversal symmetry is not violated, then the same phase is collected in both loops leading to constructive interference. The electron is located in the loop and does therefore not contribute to the current flow. This leads subsequently to an increase (decrease) in resistance (conductance).

Using a semi-classical 2-dimensional approach [50], the correction to the conductance is given by

$$
\Delta \sigma=-\frac{e^{2}}{2 \pi^{2} \hbar} \ln \left(1+\frac{\tau_{\phi}}{\tau}\right)
$$


With the momentum relaxation time $\tau$ already introduced in subsection 1.2.1 and the phase coherence time $\tau_{\phi}$. Since $\tau_{\phi}$ drastically scales with temperature $T$, the effect becomes mostly apparent only at low temperatures.

Under the influence of a magnetic field $B$ both paths collect different phases and the expression generalizes to (and assuming for $\tau_{\phi} \gg \tau$ ) [22]

$$
\Delta \sigma=-\frac{e^{2}}{2 \pi^{2} \hbar}\left[\Psi\left(\frac{1}{2}+\frac{\hbar}{4 e B D \tau}\right)-\Psi\left(\frac{1}{2}+\frac{\hbar}{4 e B D \tau_{\phi}}\right)\right]
$$

with the digamma function $\Psi(x)$. Figure 1.12 shows the changes in conductance for different momentum relaxation times $\tau$. As a consequence of decreasing $\tau$ both the absolute value of $\Delta \sigma$ [see Eq. (1.29)] and the change in magnetic field increases, since the electrons can better localize, if their free range of movement is limited.

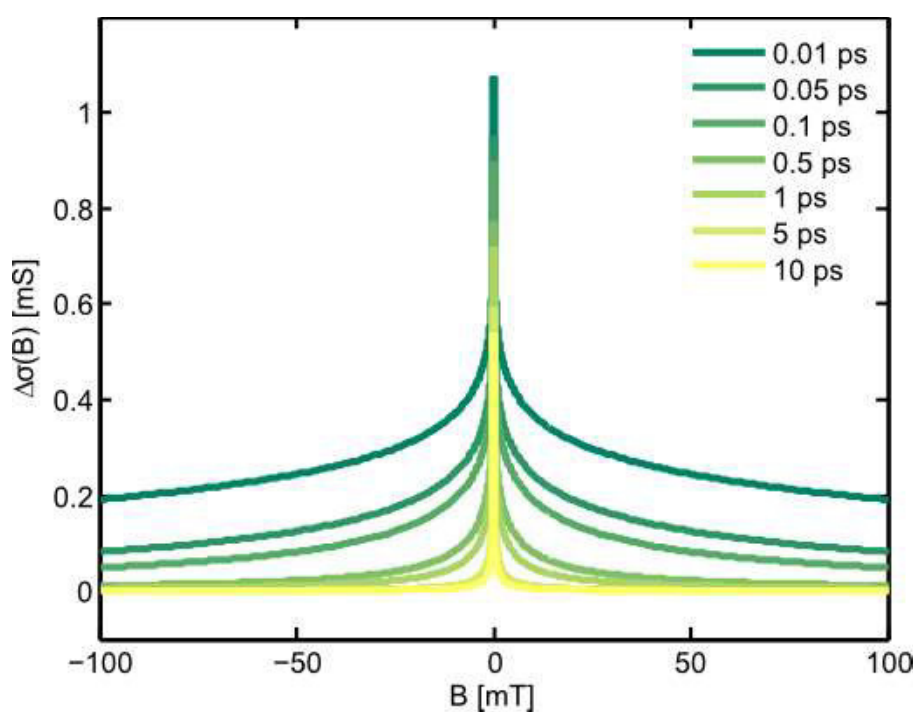

Figure 1.12: Magnetic field dependence of weak localization. Absolute change in conductance $\Delta \sigma$ as a function of magnetic field $B$. Color-coded are different values for the momentum relaxation time $\tau$ (Additional parameters: $\tau_{\phi}=10 \mathrm{~ns}, D=0.1 \mathrm{~m}^{2} / \mathrm{s}$ ).

Due to the peculiarities of graphene arising from its band structure and the pseudospin (see section 1.1) the correction in Eq. (1.30) changes and is described in the following. McCann et al. derived a theory of weak localization in graphene [123]

$$
\Delta \sigma=\frac{e^{2}}{\pi \hbar}\left[F\left(\frac{B}{B_{\phi}}\right)-F\left(\frac{B}{B_{\phi}+2 B_{i}}\right)\right]-\frac{2 e^{2}}{\pi \hbar} F\left(\frac{B}{B_{\phi}+B_{i}+B_{*}}\right)
$$


With $\quad F(x)=\ln (x)+\Psi\left(\frac{1}{2}+\frac{1}{x}\right)$

Additionally, the magnetic fields $B_{\phi, i, *}$ are related to the scattering times $\tau_{\phi, i, *} /$ scattering lengths $L_{\phi, i, *}$

$$
B_{\phi, i, *}=\Phi_{0}\left(4 \pi D \tau_{\phi, i, *}\right)^{-1}=\Phi_{0}\left(4 \pi L_{\phi, i, *}^{2}\right)^{-1}
$$

Here, $\Phi_{0}=h / 2 e$ is the flux quantum and $D$ the diffusion constant. The relaxation time $\tau$ has been replaced by the two time scales $\tau_{i}$ and $\tau_{*}$, the intervalley and the combined scattering time, respectively. They contain the scattering contributions induced by graphene's band structure. Intervalley scattering describes scattering between Dirac cones of different sublattices $\boldsymbol{K}_{ \pm}$(see section 1.1). The combined scattering time $\tau_{*}$ includes the intervalley scattering time, the intravalley scattering time $\tau_{z}$ (scattering within one sublattice $\boldsymbol{K}_{ \pm}$) and a correction due to trigonal warping $\tau_{w}$. The latter is induced by a distortion of the energy dispersion in Eq. (1.15) at higher energies with three fold symmetry enabling additional scattering. The combined scattering time is defined as $\tau_{*}^{-1}=\tau_{i}^{-1}+\tau_{z}^{-1}+\tau_{w}^{-1}$. The sign for the terms involving $B_{i, *}$ is different and thus describes anti-localization of the electrons. This weak anti-localization in graphene is a consequence of the Berry's phase of $\pi \cdot[123]$

In section 4.3 we will study the influence of single dopant atoms, characterized by scanning tunneling microscopy and spectroscopy, on magnetotransport. As it turns out the presence of this atomic scale scattering centers is triggering a transition from a classical quadratic MR (subsection 1.2.4) to a pronounced weak localization behavior as discussed in above. 



\section{Introduction to the experiments}

This chapter gives an overview of the sample preparation techniques and experimental methods used in this thesis and thus paves the way for the following chapters.

Section 2.1 introduces the sample preparation of graphene. Since its discovery, different preparation methods of graphene have been reported.[13, 103, 135, 164] Besides the initially used exfoliation ("scotch-tape") method, epitaxial-grown graphene on silicon carbide $(\mathrm{SiC})$ and graphene grown by chemical vapor deposition (CVD) belong to the most prominent methods. In most studies in the framework of this thesis we investigated graphene on SiC-graphene (in chapter 3, section 3.2 and 3.4 as well as in chapter 4) which has already been studied in our group in recent years [42, 43]. An introduction to the growth method and its history is given in section 2.1.1. In the study presented in section 3.3 in chapter 3 we used commercial graphene on $\mathrm{SiO}_{2}$ which is treated here in section 2.1.2. We used this sample system on the one hand, because the substrate is insulating already at room temperature necessary in this experiment conducted at ambient conditions. On the other hand, different kinds of defects are found in this system emerging from the differences in the growth process. Additionally, the samples used in both studies in chapter 4 have been treated with low energy ion bombardment in the group of Prof. Hans Hofsäss. In section 2.1.3 we introduce this method.

Moreover, different experimental techniques have been employed to study the local transport properties in graphene and are discussed in section 2.2. In general, scanning tunneling microscopy (STM), introduced in section 2.2.1 has been used in most studies (In chapter 3, section 3.2 and 3.4 as well as chapter 4). Additionally, scanning tunneling spectroscopy (STS) treated in section 2.2.2 was used in particular to investigate the local electronic structure of the graphene sheet as well

as doping atoms in chapter 4 , section 4.3. An introduction to scanning tunneling potentiometry is given in 2.2.3. This technique is later used in section 3.2 and 3.4 in chapter 3 to study the local voltage drop in SiC-graphene. The effect of thermovoltage is discussed in 2.2.3.1 and the experimental implantation of a magnetic field potentiometry setup in 2.2.3.3. In section 3.3 of chapter 3 we use the atomic force microscopy (AFM)-based technique of Kelvin probe force microscopy (KPFM) to investigate the voltage drop in CVD-graphene on $\mathrm{SiO}_{2}$. It is introduced in 2.2.4. For ion-implanted graphene samples we performed macroscopic transport measurements in the study in section 4.3 of chapter 4 . Therefore, an introduction to them is given in section 2.2.5. 


\subsection{Sample preparation}

\subsubsection{Epitaxial-grown graphene on SiC}

Silicon carbide is a compound semiconductor of silicon and carbon. Figure 2.1a shows the crystal structure. Here, $6 \mathrm{H}-\mathrm{SiC}$ is used for graphene preparation meaning that hexagonal $(\mathrm{H})$ stacking with 6 different SiC-bilayers (one plane of $\mathrm{Si}$ and one of $\mathrm{C}$ ) is found before repetition. [66] Two different surfaces, Si-terminated and C-terminated can be found. In this thesis we use commercial wafers from PAMXIAMEN with n-doped $6 \mathrm{H}-\mathrm{SiC}$ and a sheet resistance specified to $0.02-0.1 \Omega \mathrm{cm}$, chemically polished on the Si-terminated (0001)-direction and a miscut of $\pm 0.5^{\circ}$.

Graphene/Graphite is grown on $\mathrm{SiC}$ by thermal sublimation of $\mathrm{Si}$. This has first been demonstrated by van Bommel et al. in 1975[185] as well as Farbeaux et al in 1998[52]. Both studies showed the emerging graphite layers on the SiC substrate that could be observed in LEED-patterns. The first preparation of graphene on SiC has been achieved in the group of Walt de Heer [12] for C-terminated side and by the Seyller-group for the Si-terminated side [20, 137, 164]. Later most of graphene's unique transport properties could be shown in SiC-graphene.[13]

We proceed in discussing the essential steps of the growth process. Reviews can be found in Ref. [66, 155]. For the protocol used in our group, an in-depth discussion can be found in Ref. [42].

By heating the SiC-crystal, silicon atoms sublimate from the surface and consequently the remaining carbon atoms form layers of graphene/graphite. Before initial graphene growth, at $1100-1150^{\circ} \mathrm{C}$ a $6 \sqrt{3} \times 6 \sqrt{3} \mathrm{R} 30^{\circ}$-phase is formed [155]. This layer consists of hexagonal oriented carbon only, though it is still chemically bond to the underlying SiC-crystal and thus no graphene-like properties are observed. This layer is often referred to as buffer layer.[66] It is partially bound to the $\mathrm{Si}$ atoms of $\mathrm{SiC}$ and surface states as well as dangling bonds can be observed in photoelectron spectroscopy[157] and STM measurements.[74] At temperatures around $1250^{\circ} \mathrm{C}$ and higher graphene starts to grow. In this way epitaxially grown layers of graphene can be achieved. Temperature and growth time determine the number of graphene layers as well as the terrace size and the defect density. Figure 2.1a sketches the change in morphology. In Figure $2.1 \mathrm{~b}$ we show the growth temperature evolution for a typical growth process. Heating the sample is achieved by resistive heating. Thus, along with the temperature the current across the sample is plotted. The SiC-crystal is heated up to $1400^{\circ} \mathrm{C}$ for $120 \mathrm{~s}$ under UHV-conditions $\left(<1 \times 10^{-9} \mathrm{mbar}\right)$ resulting in monolayer graphene as well as bilayer graphene areas. Since the growth is mediated by the sublimation of $\mathrm{Si}$, additional graphene layers grow underneath existing ones.[48] The large-scale sample morphology is shown in Figure 2.1c measured in ex-situ AFM measurements. Before the growth, only 


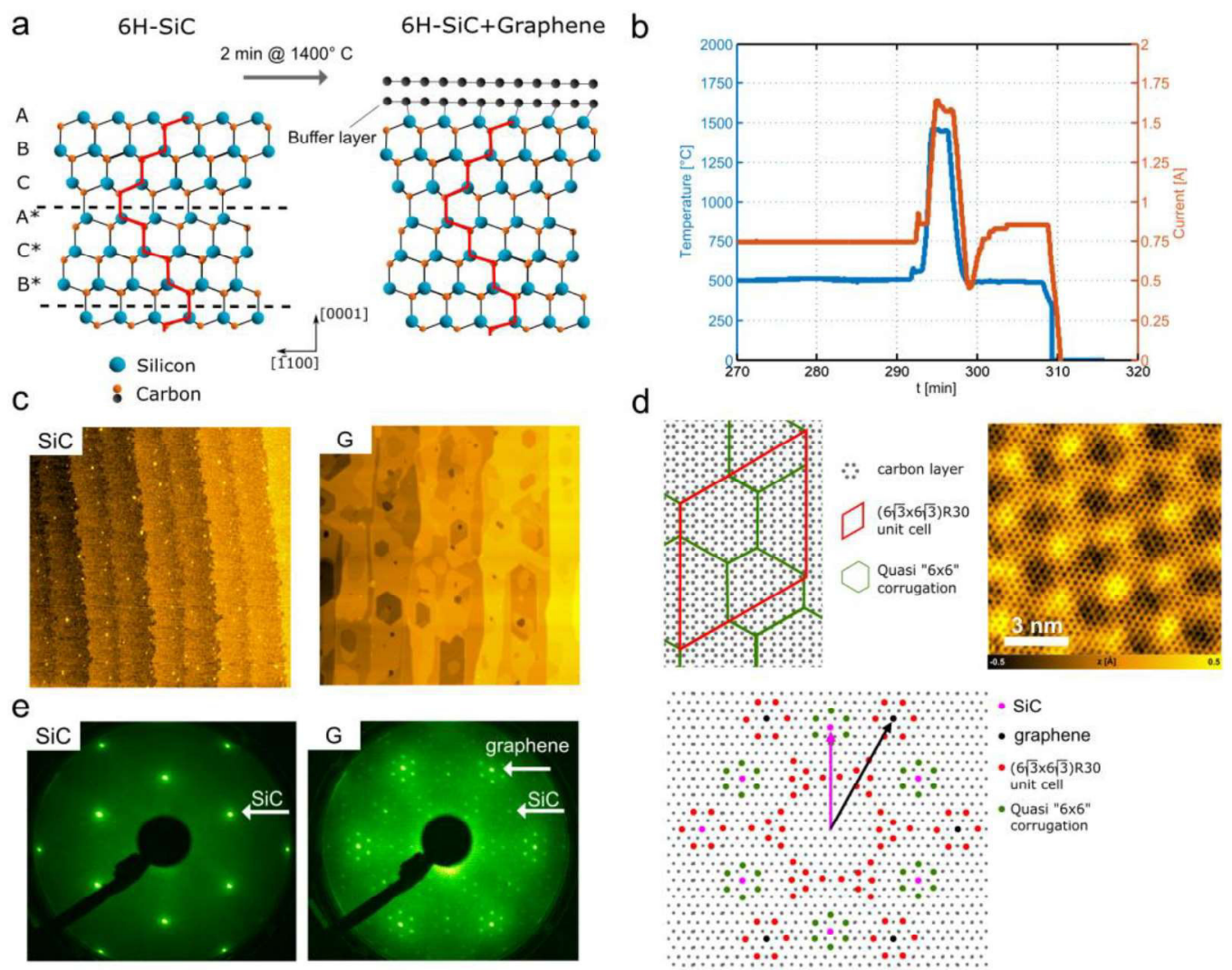

Figure 2.1: Preparation of epitaxial graphene. (a) Cross-section of the atomic structure of $6 \mathrm{H}-\mathrm{SiC}$. Heating it for $2 \mathrm{~min}$ at $1400^{\circ} \mathrm{C}$ (under UHV-conditions) leads to the formation of epitaxial-grown graphene on its surface. (b) Growth temperature during the preparation along with the current used for resistive heating of the sample as a function of time. (c) Sample surface before and after growth. Clearly hexagonal islands have formed and the morphology is more pronounced due to the graphene growth and additional step-bunching. (d) Top view schematic of epitaxial graphene showing the graphene honeycomb lattice, the unit cell of the $6 \sqrt{3} \times 6 \sqrt{3} \mathrm{R} 30^{\circ}$-reconstruction as well as the hexagons of the $6 \times 6$-quasi corrugation (Adapted from Ref. [155]). On the right: STM image showing atomic graphene lattice and the $6 \times 6$ quasi corrugation ( $V_{\text {Bias }}=50 \mathrm{mV} / I_{\text {set }}=250 \mathrm{pA}$. Taken from Ref. [200]/ section 4.3). (e) LEED-images before and after growth (Both taken at $126.6 \mathrm{eV}$ ). While for $\mathrm{SiC}$ only a six spots are visible (in first order) in a hexagonal diffraction pattern, after growth the situation is more complicated. The 6 -fold graphene spots can be identified by the different rotation and lattice constant than the SiC-spots. The additional spot features can be attributed to the additional reconstruction-grids as indicated on the right (Adapted from Ref. [155]). (data in a,b,c and e [left] are taken from Ref. [199])

small regular SiC-surface steps are present. After the growth process, several terraces and larger steps have formed. As mentioned above, the lattice mismatch between graphene and $\mathrm{SiC}$ leads to the large $6 \sqrt{3} \times 6 \sqrt{3} \mathrm{R} 30^{\circ}$-reconstruction depicted in Figure 2.1d that leads to significant buckling of the atoms [155]. For STM- 
measurements (also Figure 2.1d, UHV, $6 \mathrm{~K}$ ) often an additional quasi-6x6 corrugation is observed. An in-depth discussion of the detailed arrangement is given in Ref. [155]. While the reconstructions have been studied by scanning probe methods [42, 51, 74, 100, 118], a direct fingerprint can be seen before and after growth in LEED-measurements. Figure 2.1e shows LEED-images of the bare SiC-crystal as well as after the growth process. Here, besides the graphene peaks, additional spots emerge, that can be identified with those of the $6 \sqrt{3} \times 6 \sqrt{3} \mathrm{R} 30^{\circ}$-reconstruction.[155]

\section{a SiC step}
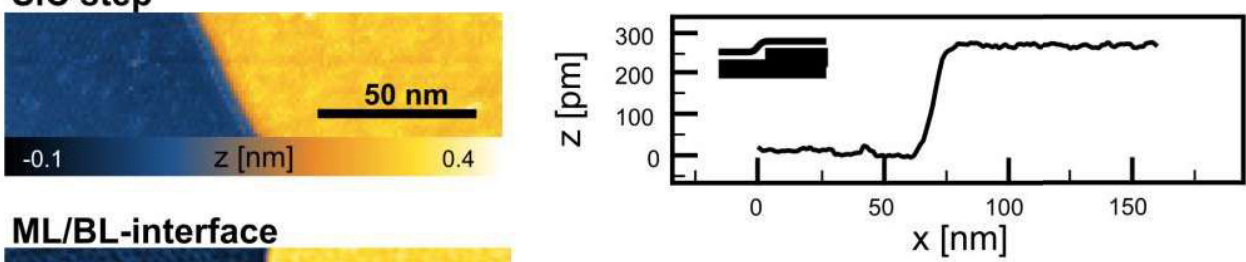

b ML/BL-interface
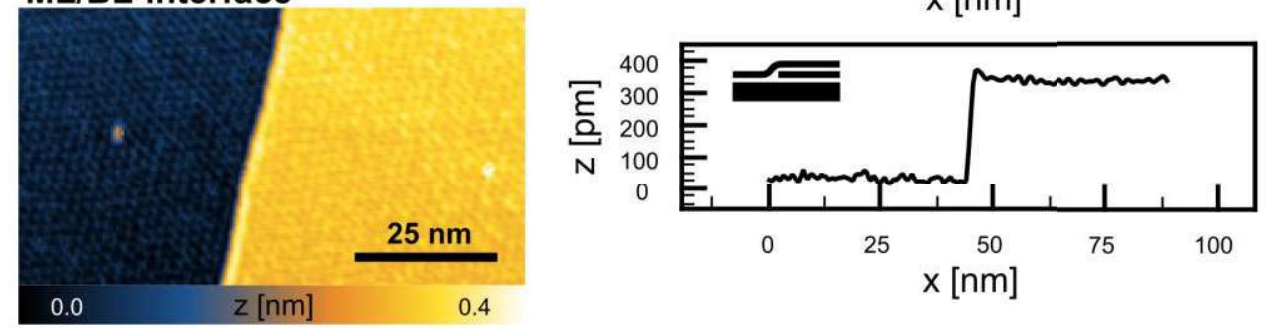

C BLG Wrinkle
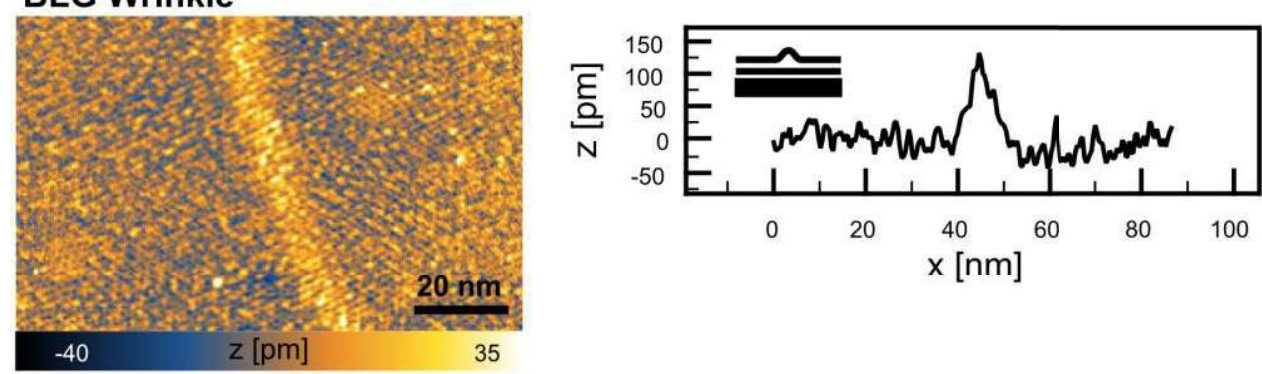

Figure 2.2: Scanning Tunneling Microscopy of structural defects in SiC-graphene. (a) Topography of SiC-substrate step $\left(V_{\text {Bias }}=-30 \mathrm{mV} / \mathrm{I}_{\mathrm{T}}=250 \mathrm{pA}\right)$ (b) a ML/BL-interface $\left(V_{\text {Bias }}=100 \mathrm{mV} / \mathrm{I}_{\mathrm{T}}=320 \mathrm{pA}\right)$ and $(\mathrm{c})$ a wrinkle on bilayer graphene $\left(\mathrm{V}_{\text {Bias }}=50 \mathrm{mV} / \mathrm{I}_{\mathrm{T}}=\right.$ $220 \mathrm{pA}$ ). On the left line sections indicate their apparent topographic height. Insets sketch the structural configuration of the defects.

In recent years the growth process has been understood in greater detail and the methods used have been refined $[66,155]$. On the one hand, while graphene was grown under UHV conditions in the beginning [164] Emtsev et al. demonstrated that large $\mu \mathrm{m}$-size terraces of MLG can be achieved by growth at atmospheric pressure in an argon atmosphere.[46] ${ }^{11}$ On the other hand, intercalation of molecular hydrogen has been used to decouple the graphene layer from the

\footnotetext{
11 In this study, we remain with UHV-growth leading to smaller graphene terraces and a higher defect density, since these defects are actually the subject of study and thus easier to find in STM measurements.
} 
underlying SiC-substrate. To do so, only the buffer layer is grown on top of SiC. Next, at elaborate temperatures between $600-1000^{\circ} \mathrm{C}$ hydrogen is saturating the bonds with the substrate and the buffer layer becomes quasi-freestanding monolayer graphene (QFMLG) [156]. Without intercalation donorlike states at the $\mathrm{SiC} / g r a p h e n e$ interface are leading to strong $n$-doping of the graphene sheet by electron transfer from the interface.[157] For monolayer (bilayer) graphene this shifts the Dirac Energy to $-440 \mathrm{mV}(-300 \mathrm{mV})$.[20, 138]

The constant improvement in synthesis of graphene films on $\mathrm{SiC}$ makes it a promising material for future device fabrication. The role of defects though remains challenging, since they are additional sources of electron scattering. As can be seen in the AFM-image in Figure 2.1c steps and interfaces are present. Figure 2.2 shows those defects as they appeare in STM measurements. We find SiCsubstrate steps (Figure 2.2a), ML/BL-interfaces (Figure 2.2b) and wrinkles on BLG (Figure 2.2c) as the dominating structural defects. They can be differentiated by their topographic height, but also by their spectroscopic features as discussed in section 3.2 and 3.4 as well as in Ref. [42]. In addition to the steps and interfaces shown in Figure 2.2, the height can also vary depending on the number of additional SiC-steps involved.

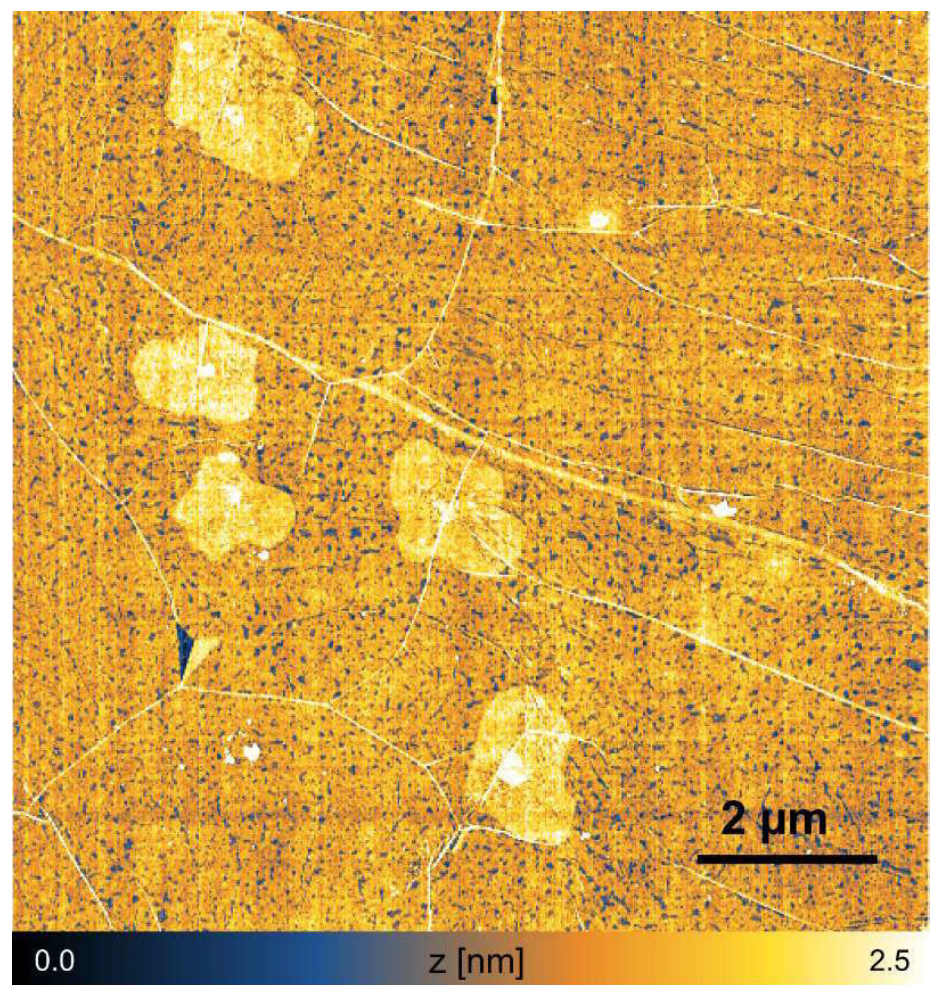

Figure 2.3: AFM-topography of CVD-grown graphene transferred to $\mathrm{SiO}_{2}$. Several defect types including bilayer graphene island, wrinkles, folded wrinkles as well as adsorbates can be identified. They are discussed in detail in section 3.3. 


\subsubsection{CVD-grown graphene on $\mathrm{SiO}_{2}$}

Another method to grow graphene is by chemical vapor deposition (CVD) on transition metal substrates and subsequent transfer to an insulating substrate (e.g. for transport measurements). In the CVD process, a gas mixture of methane and hydrogen [103] is introduced to a reactor at about $1000^{\circ} \mathrm{C}$. Thus, methane decomposes to carbon radicals on the metal surface. Then, single graphene layers start to form. Subsequently, graphene can be transferred to a different substrate.[104] This is usually been done by depositing PMMA and then etching away the metal substrate. Next, the PMMA/graphene can be placed on the substrate of choice, in this case $\mathrm{SiO}_{2}$, and the PMMA is etched away using acetone. In section 3.3 we study the local transport in commercial samples of graphene on $\mathrm{SiO}_{2}$. A typical AFM image is shown in Figure 2.3 revealing monolayer and bilayer graphene areas in addition to wrinkles and adsorbates. The structure is also discussed in more detail in section 3.3.

\subsubsection{Low-energy ion-implantation in graphene}

In this thesis incorporation of substitutional atoms into graphene was realized via mass selected ion beam deposition in the group of Prof. Hans Hofsäss at the II. Physical Institute of the University of Göttingen. This part was carried out by Julian Alexander Amani, Steffen Weikert and Prof. Hans Hofsäss. Figure 2.4 sketches the experimental accelerator setup of ADONIS (German: Anlage zur Deposition niederenergetischer lonen auf Substraten). The setup is described in greater detail in Ref. [65]. Here, we briefly discuss the main parts and the parameters needed for implantation of foreign atoms into graphene.



Figure 2.4: Schematic of the accelerator ADONIS used for low energy ion beam implantation into graphene. Taken from [65]. 
A Sidenius type hot filament hollow cathode ion source is used to produce a 30 $\mathrm{keV}$ ion beam. Subsequently, it is focused by electrostatic lenses and is run through a $90^{\circ}$ separation magnet and an aperture mass-selecting a certain $\mathrm{e} / \mathrm{m}$ ratio that allows to differentiate different isotopes. Subsequently, different types of lenses are used for focusing and to create a homogeneous beam profile. In the following the beam gets spatially modulated by a beam sweep leading to a uniform profile of $1.5 \mathrm{~cm}^{2}$ [8] and thus to a larger irradiation area. The beam is decelerated down to kinetic energies as low as $25 \mathrm{eV}$ (with a few eV wide tail towards lower energies [8]). This deceleration is necessary, so that ions are implanted in the very first layer only and not into the bulk and it is crucial for implantation into 2D-materials. The amount of charge arriving per unit area on the sample, the fluence, is measured by an electronic current integrator. In the framework of this thesis the fluence for N-/B- and C-ion implantation was chosen to be $\sim 10^{14} \mathrm{~cm}^{-2}=1 \mathrm{~nm}^{-2}$.

For the implantation, the epitaxial graphene samples are fixed on a copper plate using conductive silver. Irradiation is taking place at a base pressure of $\approx$ $10^{-8} \mathrm{mbar}$ and at room temperature.

\subsection{Methods}

\subsubsection{Scanning Tunneling Microscopy}

The scanning tunneling microscope (STM) was first demonstrated by Gerd Binnig and Heinrich Rohrer in 1982[18]. It was the first method within the expanding family of scanning probe methods and only 4 years later, in 1986, both received the Nobel Prize in physics for their groundbreaking discovery giving access to the nanoscale in real space.

The working principle is based on the effect of quantum tunneling: By bringing a sharp metal tip in close proximity to a conductive sample, a finite tunnel current $I_{T}$ will flow through the vacuum barrier between tip and sample, if a bias voltage is applied. This tunneling current drops exponentially with distance $z$. Therefore, the tip is often $\lesssim 1 \mathrm{~nm}$ away from the sample [24]. Using piezo crystals this height can carefully be adjusted and the spatial coordinates $(x, y)$ can be scanned.

These sophisticated experiments require a high stability of the tunnel junction. Therefore, excellent damping of vibrations is needed. Moreover, clean samples are achieved by UHV-preparation; low temperatures ensure thermal stability.

In the framework of this thesis experiments have been conducted in two Besocke type low-temperature $(6 \mathrm{~K})$ STMs mounted on the bottom of a liquid helium bath cryostat, one equipped with an additional $6 T$ superconducting magnet coil. The 
details of our STM setup can be found in Ref. [159]. An in-depth introduction to STM-theory and -experiment is given in Ref. [19, 24].

\subsubsection{A brief history of STM theory}

A first description often used to introduce of the physics of an STM is found in the work by Bardeen in 1961, who investigated electron tunneling for a system of planar tunnel junctions treated in a time-dependent perturbation theory approach [10], even before the realization of an STM [18]. Studying two metal layers separated by an insulating oxide, he formulated an expression for the tunnel current $I_{T}$ between them when a bias voltage $V$ is applied. Starting with a set of wavefunction $\psi_{v}$ and $\psi_{\mu}$ in sample and tip, respectively, the transition matrix elements can be calculated by $[10,177]$

$$
M_{\mu \nu}=-\frac{\hbar^{2}}{2 m} \int\left[\psi_{\mu}^{*} \nabla \psi_{\nu}-\psi_{\nu} \nabla \psi_{\mu}^{*}\right] d \boldsymbol{S}
$$

Here, the expression in parentheses then is the current operator. Integration is taken over the surface $\mathrm{S}$, separating the two surfaces for the transition from state $v$ to $\mu$. The tunnel current is then given by $[19,177]$

$$
\begin{aligned}
I_{T}(V) & =\frac{2 \pi e}{\hbar} \sum_{\mu, \nu}\left[f\left(E_{\mu}\right)-f\left(E_{v}\right)\right]\left|M_{\mu \nu}\right|^{2} \delta\left(E_{\nu}+V-E_{\mu}\right) \\
& \approx \frac{2 \pi}{\hbar} e^{2} V \sum_{\mu, \nu}\left|M_{\mu \nu}\right|^{2} \delta\left(E_{\mu}-E_{F}\right) \delta\left(E_{\nu}-E_{F}\right)
\end{aligned}
$$

where $f(E)$ is the Fermi-Dirac distribution function and $V$ the applied bias voltage. In the limit of small $V$ and low temperatures, the summands are the transfer rates given by Fermi's golden rule (second row).[19]

Assuming that the transition matrix element $M_{\mu \nu}$ is a function of energy only, the sum can be replaced by an integral over the the density of states (DOS) of tip $\rho_{T}(E)$ and sample $\rho_{S}(E)$.[24]

$$
I_{T}(V) \propto \int\left[f_{s}(\varepsilon-e V)-f_{t}(\varepsilon)\right] \rho_{S}(\varepsilon-e V) \rho_{T}(\varepsilon)|M(\varepsilon, e V)|^{2} d \varepsilon
$$

The special geometry encountered in an STM experiment, a sharp spherical tip, is accounted for in the Tersoff-Hamann model. They modelled the wave function of the tip as a spherical S-like wave function centered at position $\boldsymbol{r}_{\mathbf{0}}$, the apex of the tip. They then find that in the limit of small voltages $V$ and small temperatures $T$ [177] 


$$
I_{T}(V) \propto V \rho_{S}\left(E_{F}, \mathbf{r}_{\mathbf{0}}\right)
$$

Thus, the tunneling current is proportional to the applied bias voltage $V$ and the sample LDOS $\rho_{S}$.

For higher voltages the matrix elements $M(\varepsilon, e V)$ cannot assumed to be constant anymore. Thus, in the Hamers model of tunneling it is replaced in Eq. (2.3) by an energy- and distance-dependent transmission $|M(\varepsilon, e V)|^{2} \rightarrow T(\varepsilon, e V, z)$

$$
\begin{gathered}
I_{T}(V) \propto \int\left[f_{s}(\varepsilon-e V)-f_{t}(\varepsilon)\right] \rho_{S}(\varepsilon-e V, r) \rho_{T}(\varepsilon, r) \mathrm{T}(\varepsilon, \mathrm{eV}, \mathrm{z}) d \varepsilon \\
\approx \int_{0}^{e V} \rho_{S}(\varepsilon-e V, r) \rho_{T}(\varepsilon, r) \mathrm{T}(\varepsilon, \mathrm{eV}, \mathrm{z}) d \varepsilon
\end{gathered}
$$

Hamers found using the Wentzel-Kramers-Brillouin (WKB) approximation for the transmission the following expression. [64]

$$
T(\varepsilon)=\exp \left(-\frac{2 z \sqrt{2 m}}{\hbar} \sqrt{\frac{\phi_{S}+\phi_{T}}{2}+\frac{e V}{2}-\varepsilon}\right)
$$

where we introduced the tip (sample) work function $\phi_{T}\left(\phi_{S}\right)$. The distance $z$ between tip and sample is the crucial parameter inducing the exponential decay of the tunneling current with distance.

\subsubsection{Constant Current Topography}

In constant current topography a fixed bias voltage $V$ is applied to the sample. Subsequently, the tunneling current $I_{T}(V)$ is adjusted to a setpoint value. While the tip is now scanned in $(x, y)$ direction, at each point the current is controlled via a closed feedback loop. Thus, surfaces of constant current are mapped. For the Hamers model in Eq. (2.3) and (2.5) this can be interpreted as a surface of constant integrated LDOS in the range $[0, \mathrm{eV}]$. Therefore, the contrast in this regions can have two reasons, physical differences in height such as atomic steps as well as electronic contrast stemming from changes in $\rho_{S}(E)$.

\subsubsection{Scanning Tunneling Spectroscopy}

To combine the high spatial with high-energy resolution, scanning tunneling spectroscopy can be used. This can be achieved by introducing the differential conductance $d I_{T} / d V(V)$. Rewriting the derivative of equation (2.5) we find [64] 


$$
\frac{d I_{T}}{d V}(V) \propto \rho_{S}(e V, r) \rho_{T}(0, r) \mathrm{T}(\mathrm{eV}, \mathrm{V}, \mathrm{z})+\int_{0}^{e V} \rho_{S}(\varepsilon, r) \rho_{T}(\varepsilon-\mathrm{eV}, \mathrm{r}) \frac{\mathrm{dT}(\varepsilon, \mathrm{eV}, \mathrm{z})}{\mathrm{dV}} d \varepsilon
$$

Here, we also approximated the result for low temperatures allowing to omit the Fermi functions. Assuming the LDOS of the tip $\rho_{T}$ as well as the transmission probability $\mathrm{T}$ to be constant in energy ${ }^{12}$, the equation simply writes

$$
\frac{d I_{T}}{d V}(V) \propto \rho_{S}(e V)
$$

and then, the differential conduction is simply proportional to the LDOS of the sample $\rho_{S}$. The assumptions made above are valid, if the energy $\varepsilon$ is small compared to the work functions of tip and sample [See Eq. (2.6)] and if the tip LDOS $\rho_{T}$ is featureless. Due to the additional contributions, the absolute value of the spectroscopy often is difficult to interpret. However, since measurements are spatially resolved, spectroscopies for different topographic features can be compared. This will be used in particular in section 4.3 for single nitrogen and boron atoms implanted into graphene with respect to the graphene sheet. While the $d I_{T} / d V(V)$ can be taken a by lock-in technique [148], all spectroscopies shown in this thesis are simply obtained by the numerical derivative of $I_{T}(V)$-curves.

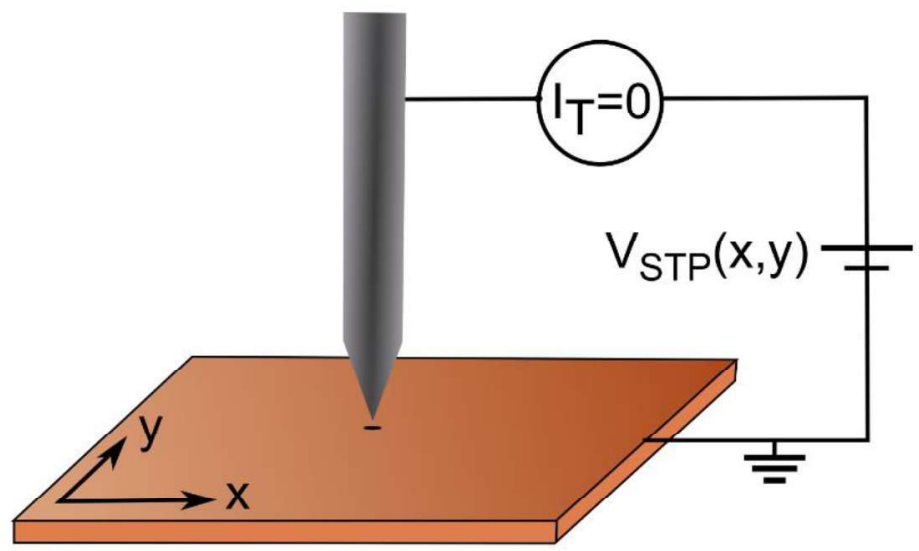

Figure 2.5: Scanning tunneling potentiometry: Without applied bias voltage $V_{\text {Bias }}$ the tunneling current is nullified in the the tunneling junction $\mathrm{I}_{\mathrm{T}}=0$. The required voltage $\mathrm{V}_{\mathrm{STP}}(x, y)$ is recorded and mapped as a function of position. Adapted from Ref. [49].

\subsubsection{Scanning Tunneling Potentiometry}

Scanning tunneling potentiometry has been established as a versatile tool to enter both the electronic (thermovoltage) and transport properties (transport STP) of a sample on the nano scale. A detailed treatment of the theory and the interpretation of the measured quantities can be found in Ref. [42, 49, 72].

\footnotetext{
12 This might not always be the case. However, as Hamers describes it, the transmission probability then "contributes a smoothly varying background".[64]
} 
The basic principle for scanning tunneling potentiometry (STP) is shown in Figure 2.5. Similar to a spectroscopy measurement, it is taken after a topography map, that is, at a certain $z$-height (fixed by the set point current) at every point $(x, y)$. Then the bias voltage $V_{\text {Bias }}{ }^{13}$ is switched off and a potential $V_{S T P}(x, y)$ is adjusted by a closed feedback-loop on the side of the tip, so that the tunneling current is zero $I_{T}=0$. At first glance, there should not be any signal expected under these conditions $\left(V_{\text {Bias }}=0 \mathrm{~V}\right)$. Two effects however lead to a finite current: thermovoltage and a transport cross voltage. Both are discussed in the following.

\subsubsection{Thermovoltage measurements}

The thermovoltage in an STM junction is a thermoelectric effect similar to the macroscopic Seebeck-effect.[49] Here, a diffusion driven current is found between two metals with different temperatures. In our STM tunnel junction the thermovoltage results from a temperature difference between tip and sample. Revisiting Eq. (2.5), we can write it slightly modified

$$
\mathrm{I}_{\mathrm{T}} \propto \int_{-\mathrm{eV} / 2}^{\mathrm{eV} / 2}\left[\mathrm{f}_{\mathrm{S}}\left(\varepsilon-\frac{\mathrm{eV}}{2}, T_{S}\right)-\mathrm{f}_{\mathrm{T}}\left(\varepsilon+\frac{\mathrm{eV}}{2}, T_{T}\right)\right] \rho_{\mathrm{S}}(\varepsilon-\mathrm{eV} / 2) \rho_{\mathrm{T}}\left(\varepsilon+\frac{\mathrm{eV}}{2}\right) T(\varepsilon+e V) \mathrm{d} \varepsilon
$$

by symmetrizing the boundaries of the integral. Both fermi functions of sample and tip are at the temperatures $\mathrm{T}_{\mathrm{S}}$ and $\mathrm{T}_{\mathrm{T}}$, respectively. In our case, the tip is assumed

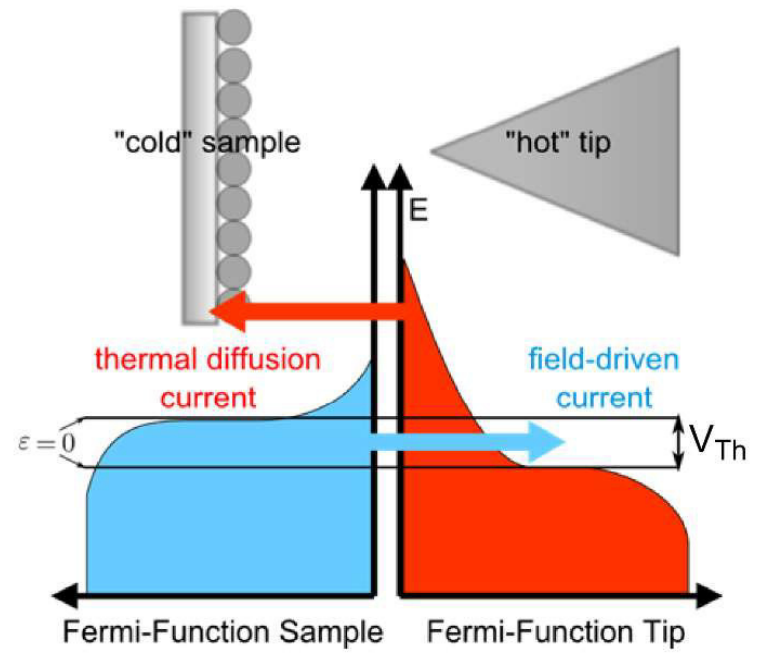

Figure 2.6: Thermovoltage in the STM junction: Due to the temperature difference between tip and sample a thermal diffusion current is induced that can be compensated by an additional applied voltage, the thermovoltage $\mathrm{V}_{\mathrm{th}}$. Adapted from Ref. [49], taken from Ref. [199].

\footnotetext{
${ }^{13}$ The subscript has been added here in contrast to the last chapter to distinguish several voltages discussed in the following.
} 
to be hotter, since it is only connected via silver wires to the helium bath [159], while the sample is directly thermally connected to the bath cryostat $(\approx 6 \mathrm{~K})$. Figure 2.6 depicts the experimental situation. Due to the temperature difference, the Fermi functions are non-equally populated and thus generate a thermally driven tunneling current $\mathrm{I}_{\mathrm{T}}$. Solving Eq. (2.9) for $\mathrm{I}_{\mathrm{T}}=0$, we obtain the voltage needed to compensate for the thermally driven current. This was solved by Stovneng and Lipavský in first-order leading to [168]

$$
V_{\text {th }}=\frac{\pi^{2} k_{B}^{2}}{6 e}\left(T_{T}^{2}-T_{S}^{2}\right)\left[\left.\frac{1}{\rho_{S}(E)} \frac{d \rho_{S}(E)}{d E}\right|_{E=E_{F}}+\left.\frac{1}{\rho_{T}(E)} \frac{d \rho_{T}(E)}{d E}\right|_{E=E_{F}}+\frac{z}{\hbar} \sqrt{\frac{2 m}{\phi}}\right]
$$
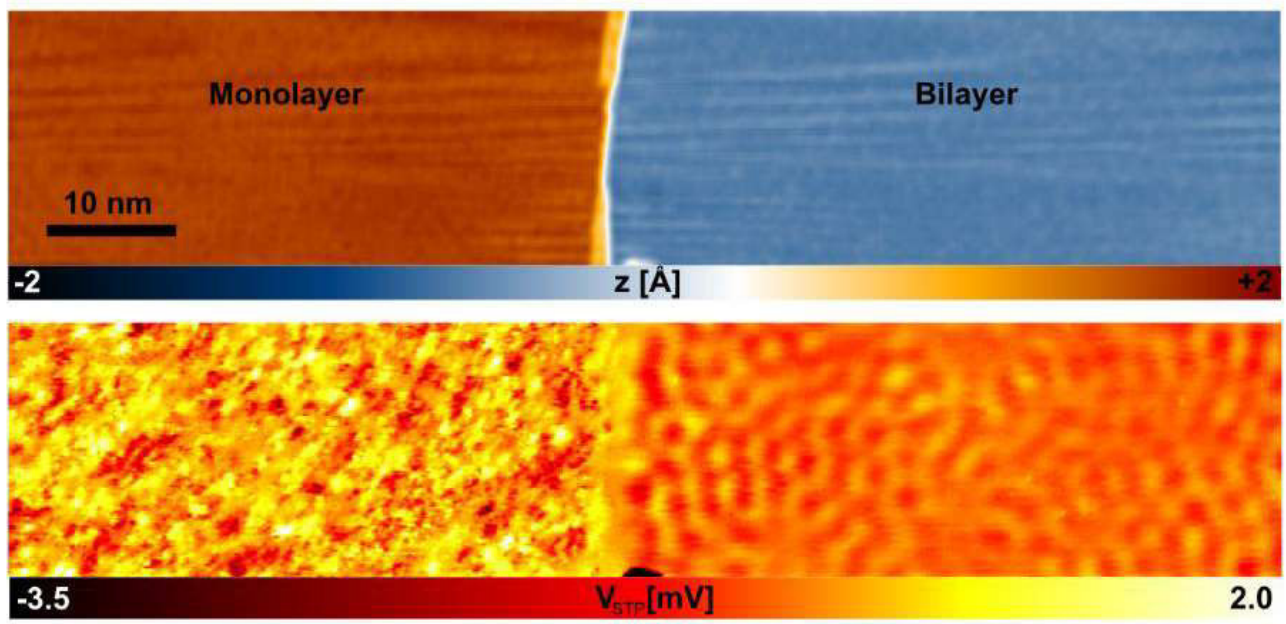

Figure 2.7: Thermovoltage measurement: Thermovoltage map $V_{\text {STP }}(x, y)$ of a monolayer/bilayer interface of SiC-graphene $\left(100 \mathrm{~nm} \times 18 \mathrm{~nm}, \mathrm{~V}_{\text {Bias }}=-50 \mathrm{mV} / \mathrm{IT}_{\mathrm{T}}=200 \mathrm{pA}\right)$. Electronically, bilayer graphene shows standing waves as interference patterns due to quasiparticle scattering, the monolayer shows disorder from the underlying buffer layer. Adapted from [96].

Thus, the thermovoltage is proportional to the difference of the temperature squares of tip and sample $\left(\mathrm{T}_{\mathrm{T}}^{2}-\mathrm{T}_{\mathrm{S}}^{2}\right)$. Moreover, while the second term in the brackets is not changing for a constant tip LDOS $\rho_{\mathrm{T}}$ and the third term containing the tipsample distance $z$ is found to be small, in particular for constant current mode ${ }^{14}$, we can approximate

$$
\left.V_{t h} \propto \frac{\pi^{2} k_{B}^{2}}{6 e}\left(T_{T}^{2}-T_{S}^{2}\right) \frac{1}{\rho_{S}(E)} \frac{d \rho_{S}(E)}{d E}\right|_{E=E_{F}}
$$

\footnotetext{
${ }^{14}$ Distance-dependent thermovoltage studies have found a maximum change of $\approx 2 \mathrm{mV} / \mathrm{nm}$ [116, 167], often though much less. Thus, for a spatially resolved thermovoltage map taken in constant current mode, where the tip is held rather constant across a surface with changes in the picometer range (see e.g. Figure 2.7), this effect is negligible.
} 
Therefore, the thermovoltage gives access to spectroscopic information, namely to the derivative of the sample LDOS $\rho_{\mathrm{S}}$ precisely at the Fermi energy $\mathrm{E}_{\mathrm{F}}$. A clear disadvantage compared to STS is the limitation to one energy $\mathrm{E}_{\mathrm{F}}$ only. In contrast, the sensitivity due to the derivative as well as the feedback loop used for data acquisition is an advantage. Figure 2.7 shows a thermovoltage map taken for a monolayer/bilayer transition of graphene on $\mathrm{SiC}$. It nicely resolves the quasiparticle interference ${ }^{15}$ observed in STS on the bilayer [117] as well as the electronic disorder due to the buffer layer for monolayer graphene.

\subsubsection{Transport STP}

Transport STP is the traditional definition of scanning tunneling potentiometry. The term has been introduced here only to distinguish it from thermovoltage measurements. The first STP setup (with transport across the sample) has been realized by Muralt and Pohl in 1986.[125] To do so, a macroscopic transport voltage $\mathrm{V}_{\text {transport }}$ is applied across the sample and $\mathrm{V}_{\mathrm{STP}}(x, y)$ is adjusted at the position of the tip. Our experimental setup is sketched in Figure 2.7 (See also Figure 3.4). Thus, the tip probes the local electrochemical potential defined already in subsection 1.2.2.

$$
\mu_{E C P}(x, y)=\mu(x, y)-e \phi(x, y)
$$

from which also the Fermi function $f(E)$ or $f(\boldsymbol{k})$ can be defined.

For an external applied bias $V_{\text {transport }}$ the system is in non-equilibrium and subsequently the distribution function changes. Within the Drude-Sommerfeld-model introduced in chapter 1 , this can be described as a shift in k-space, leading to differently populated functions $f\left(\boldsymbol{k}^{+}\right)$and $f\left(\boldsymbol{k}^{-}\right)$which we now treat separately. The respective electrochemical potentials then can be written as

$$
\begin{aligned}
& \mu_{E C P}^{\boldsymbol{k}^{+}}(x, y)=\mu^{\boldsymbol{k}^{+}}(x, y)-e \phi(x, y) \\
& \mu_{E C P}^{\boldsymbol{k}^{-}}(x, y)=\mu^{\boldsymbol{k}^{-}}(x, y)-e \phi(x, y)
\end{aligned}
$$

A tunneling tip that is now brought close to the sample senses both distributions. A simple superposition has been postulated by Datta [31]

\footnotetext{
${ }^{15}$ Quasiparticle interference patterns emerge, if an electron can scatter either within a Dirac cone or within two cones of the same sublattice (intravalley scattering) or between cones of different sublattice (intervalley scattering). The difference in initial and final k-vector then becomes visible in standing wave patterns. The long wavelength oscillations on the bilayer in Figure 2.7 is strongly suppressed on the ML due to the pseudospin conservation and the suppression of direct backscattering. Quasiparticle interference patterns in epitaxial graphene on SiC is described in great detail in Ref. [117].
} 


$$
\mu_{S T P}(x, y)=\frac{1}{2}\left[\mu_{E C P}^{k^{+}}(x, y)+\mu_{E C P}^{k^{-}}(x, y)\right]
$$

In the limiting case of fully diffuse transport $e \phi(x, y)$ changes only as expected from Drude-Sommerfeld theory (section 1.2.1) [72]. For other cases, the changes in the chemical potential also contribute as discussed for the Landauer-Residual Resistivity Dipole in section 1.2.2.

The two contributions, thermovoltage and transport STP are measured simultaneously. In general this can lead to the evaluation of incorrect values for the voltage drops, if both effects are comparable and a transition of two areas of different LDOS (and thus different thermovoltage) is analyzed, e.g. a monolayer-bilayer interface (see Figure 2.7). In chapter 3, subsection 3.2.7.3 we introduce a way to disentangle both contributions using datasets of opposite current direction and voltage drop.

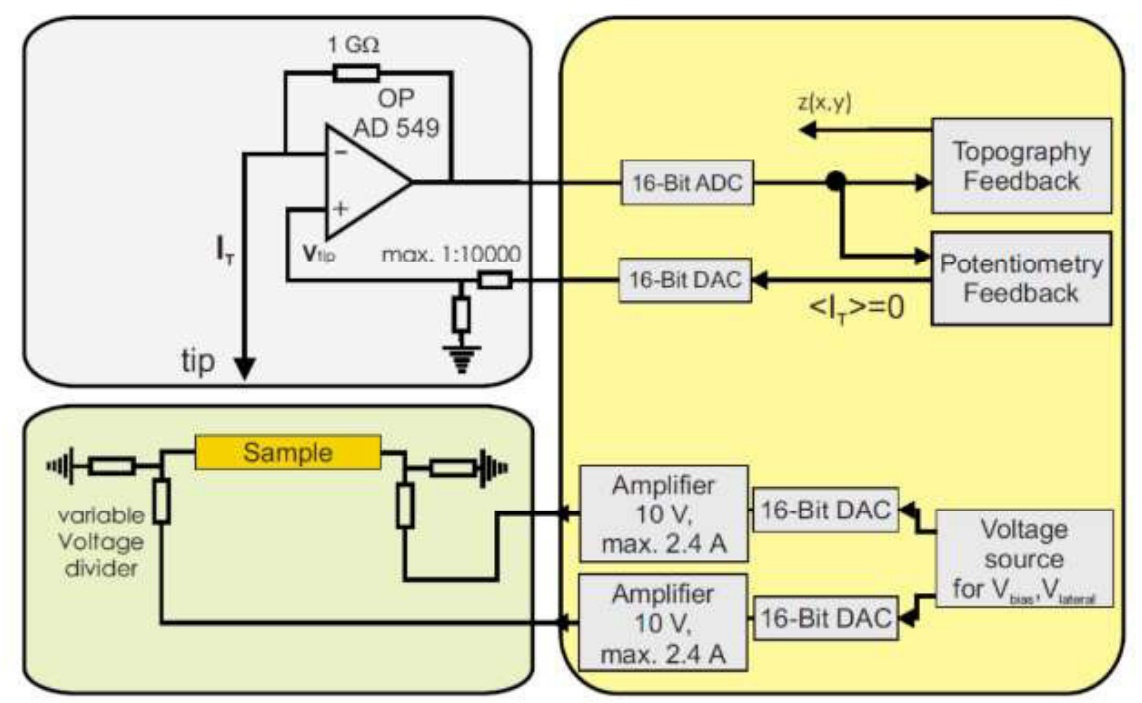

Figure 2.8: Versatile high resolution scanning tunneling potentiometry implementation: On the sample side two digital-analog-converter (DAC) can apply a transport voltage $V_{\text {Transport }}$ across the sample. On the tip side, the potential can be locally probed by reading out the current via an analog-digital-converter (ADC). An additional feedback loop then adjusts the STP voltage $V_{S T P}$ connected to the add-input of the operational amplifier so that $I_{T}=0$. Reprinted (adapted) with permission from Ref. [41]. Copyright (2010) AIP Publishing LLC.

In recent years different setups have been developed for STP $[9,41,63,112,160]$. In general, two main approaches exist to realize STP. Either in a 4-point probe setup, where two movable STM tips inject a current, the third acts as a reference voltage probe and the fourth is used for scanning [11, 112, 122]. In addition, a 
scanning electron microscope can be used for locating the tip. An overview is given in Ref [102].

The other approach uses fixed contacts that are brought onto the prepared sample, in our case ex-situ. In the 4-point probe (4PP) approach samples can directly be investigated without worrying about making contacts or use of lithography. Additionally, the 4 voltage probes can already analyze the transport on a larger scale by recording distance dependent IV-curves [85, 102]. In contrast, most 4PP experimental setups can only operate at room temperature/liquid nitrogen temperature leading to a lack of thermal stability. Moreover, no design including a magnetic field has been introduced yet.

Adding external contacts onto our samples allows us to use our conventional STM setup [159] and therefore achieve low noise and high stability during imaging at liquid helium temperature.

\subsubsection{Magnetic Field STP}

To allow for the measurements presented in chapter 3 , section 3.4 an STM equipped with an additional $6 \mathrm{~T}$-magnet has been used for the first time in our group. The microscope was developed priorly while in the framework of this thesis the setup was finalized, electronics and cabling were installed and it was put into operation allowing for the first magnetotransport STP experiments. The setup is shown in Figure 2.9 with all its components. The main features are a liquid helium cryostat allowing measurements at low temperatures $(6 \mathrm{~K})$ as well as a superconducting coil magnet that enables us to create a strong magnetic field at the position of the sample. Due to the high stability of the system the magnetic field can be changed while staying in tunneling contact between tip and sample. This allows us to take STP measurements at the same position as a function of the magnetic field.
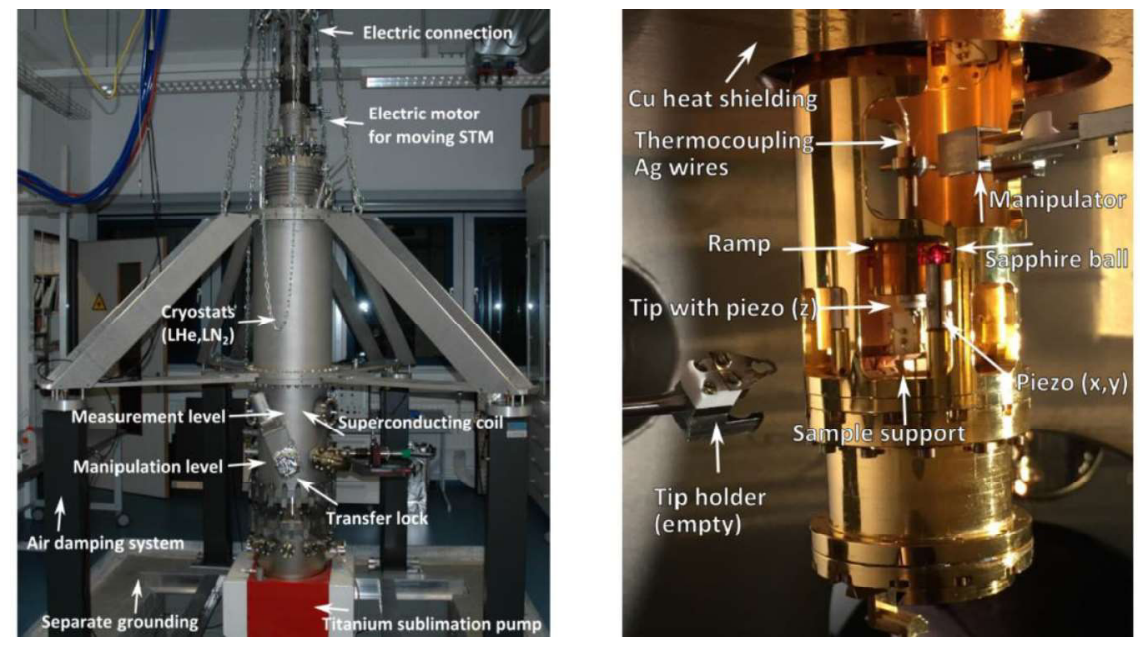

Figure 2.9: 6T-STM setup. (a) Complete setup of our low temperature STM with optional magnetic field. (b) STM in the interior of the UHV chamber with empty tip holder. Taken from Ref. [96]. 


\subsubsection{Atomic Force Microscopy}

Atomic Force Microscopy (AFM) is another member in the family of scanning probe methods and was invented by Gerd Binnig later on in 1986 [17]. In contrast to STM, AFM utilizes the force interaction between tip and sample to get spatial resolved information about a sample. However, common ground is the use of piezo-electric elements for scanning and tip height as well as a closed feedback loop (In conventional topography mode).

In AFM a cantilever is brought into mechanical vibration close to its resonance frequency. As can be seen in Figure 2.10, a thin sharp tip is located at the end of the cantilever. When the tip is now approaching the sample different forces are acting on it. The total force acting on the tip leads to a change in frequency, phase and amplitude of the cantilever.[172, 197] These changes can be detected by a laser beam focused on the back side of the cantilever. The reflected beam is then read out by a photo diode.

Typically, the interaction is dominating by attractive Van der Waals forces at large distances and Pauli repulsion at close proximity, both combined in the description of a Lennard-Jones-potential. However, in general any kind of force able to change the behavior of the tip can be measured including magnetic, electric and mechanical interaction.[172, 197]

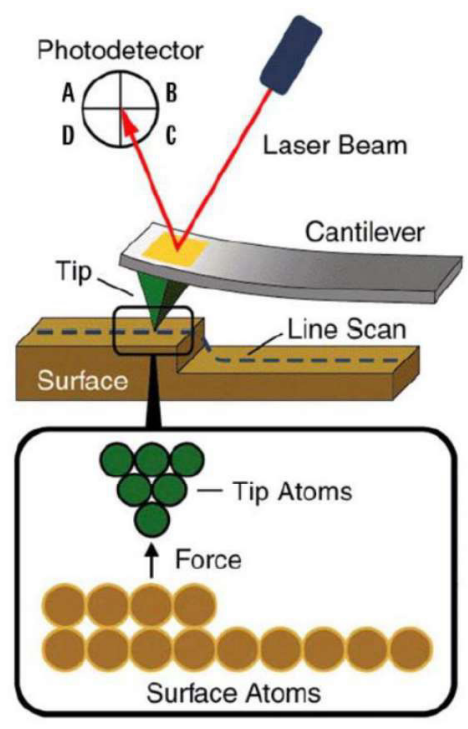

Figure 2.10: Schematic of an atomic force microscope. A scanning tip is moved across a sample surface while being brought into mechanical vibration. The changes in frequency, phase and amplitude of the oscillation due to force interaction with the surface are detected by a reflected laser beam and a photo detector. Taken from Ref. [172].

The setup used in this thesis is a commercial Agilent 5600LS AFM operating at room temperature and ambient conditions. 


\subsubsection{Topography Mode}

To access spatially resolved structural information about a sample, in general different modes can be used in an AFM. Contact mode, non-contact mode and intermittent contact mode are acting in different regimes of the Lennard-Jones potential. In the intermittent contact mode (tapping mode) used for the data taken in the framework of this thesis, the cantilever is driven to oscillate with an amplitude into a regime governed by both long-range attractive and short-range repulsive forces.[172] The feedback loop adjusts now for same amplitude of the oscillation, whereas the z-height of the tip is adjusted and mapped as the topography. Thus, by moving across an atomic step as shown in Figure 2.10 the tip has to approach/retract to compensate for the difference in amplitude caused by the distance-dependence of the force.

\subsubsection{Kelvin Probe Force Microscopy}

In Kelvin probe force microscopy (KPFM), the contact potential difference between tip and sample manifested as an electrostatic force can be measured (in addition to the topography). Using a conductive tip electrons will flow between tip and sample until both Fermi-energies are on the same Fermi-level. This is reflected in the different work functions of tip $\left(\Phi_{t}\right)$ and sample $\left(\Phi_{s}\right)$ defining the contact potential difference [173]

$$
V_{C P D}(x, y)=\frac{1}{e}\left[\Phi_{t}-\Phi_{s}(x, y)\right]
$$

To measure it, both a DC bias voltage $V_{D C}$ as well as a small AC modulation $V_{A C}$ are applied to the tip with respect to the sample leading to

$$
V=V_{D C}-V_{C P D}+V_{A C} \sin \left(\omega_{2} t\right)
$$

The force measured in this experiment

$$
F=\frac{1}{2} \frac{d C}{d z} V^{2}
$$

where $C$ is the capacity, can then be written as $F=F_{D C}+F_{\omega_{2}}+F_{2 \omega_{2}}$ with

$$
\begin{gathered}
F_{D C}=\frac{1}{2} \frac{d C}{d z}\left[\left(V_{D C}-V_{C P D}\right)^{2}+\frac{1}{2} V_{A C}^{2}\right] \\
F_{\omega_{2}}=\frac{d C}{d z}\left[V_{D C}-V_{C P D}\right] V_{A C} \sin \left(\omega_{2} t\right) \\
F_{2 \omega_{2}}=-\frac{1}{4} \frac{d C}{d z} V_{A C}^{2} \cos \left(2 \omega_{2} t\right)
\end{gathered}
$$


Apparently, $F_{\omega_{2}}$ vanishes for $V_{D C}=V_{C P D}$. Therefore, $V_{C P D}$ is detected by compensating for $F_{\omega_{2}}$ at frequency $\omega_{2}$. The amplitude detection is realized by a Lock-In measurement and $V_{D C}$ is adjusted in a closed feedback loop. Since $V_{C P D}$ is obtained by nullifying the amplitude of $F_{\omega_{2}}$, it is referred to as amplitude modulated Kelvin Force Microscopy (AM-KFM). It is the mode used in this thesis. Alternatively, the signal can be detected via frequency modulation (FM-KFM).[173]

To study the transport properties of graphene, our group has combined the KPFMsetup with a transport voltage $V_{\text {Transport }}$ across the sample.[43, 174]

$$
\mathrm{V}_{\text {Kelvin }}(\mathrm{x}, \mathrm{y})=\mathrm{V}_{\mathrm{CPD}}(\mathrm{x}, \mathrm{y})+\mathrm{V}_{\text {Transport }}(\mathrm{x}, \mathrm{y})
$$

Thus, besides the contact potential difference in Eq. (2.15), the tip is also sensitive to the electrostatic potential drop across the sample induced by the transport voltage.

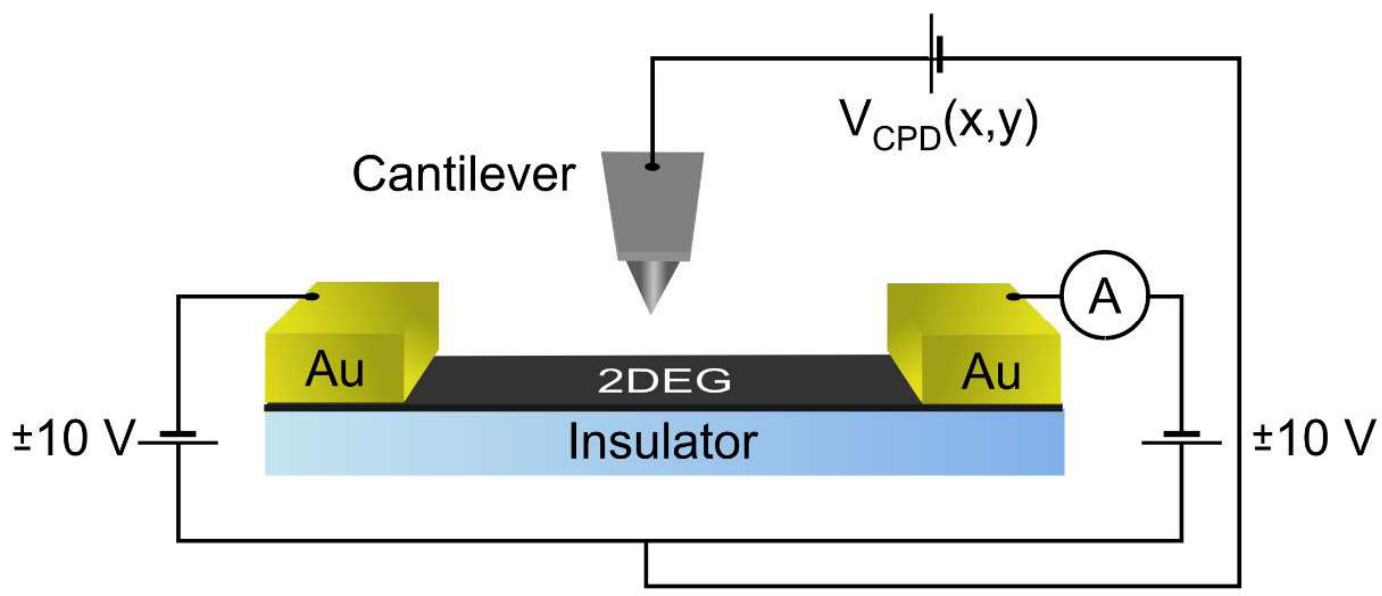

Figure 2.11: Cross-voltage KPFM setup. The sample is connected in two-terminal geometry. The contact potential difference is compensated by an additional voltage $V_{C P D}(x, y)$ on the tip side. Each gold contact is connected via aluminum bonds to a power supply. Moreover, the current is simultaneously measured in series.

Prior to this thesis, this enabled us to determine the graphene sheet resistance as well as the contact resistance between graphene and gold contacts.[43] In the framework of this thesis, this setup has been extended and improved allowing us to resolve the voltage drop at local defects, presented in section 3.3. The setup is shown in Figure 2.11. The sample is contacted with two gold contacts. Each one is connected to a voltage supply operating in the range of $\pm 10 \mathrm{~V}$. Both polarities are necessary to allow opposite current flow through the sample. Both voltage supplies have an optional voltage amplifier, a current meter is connected in series. Moreover, we show in section 3.3 how to disentangle contributions arising from changes in $\mathrm{V}_{\mathrm{CPD}}(\mathrm{x}, \mathrm{y})$ and $\mathrm{V}_{\text {Transport }}(\mathrm{x}, \mathrm{y})$. 


\subsubsection{Transport measurements}

To perform transport measurements on macroscopic sample areas $\left(\approx 1 \mathrm{~mm}^{2}\right)$ we use a physical property measurement system (PPMS) developed by Quantum Design located in the I. Physical Institute in Göttingen. This system is able to vary the temperature in a range of $1.9 \mathrm{~K}-400 \mathrm{~K}$ and to apply magnetic fields up to $\pm 9 \mathrm{~T}$.

Figure 2.12 sketches the setup.
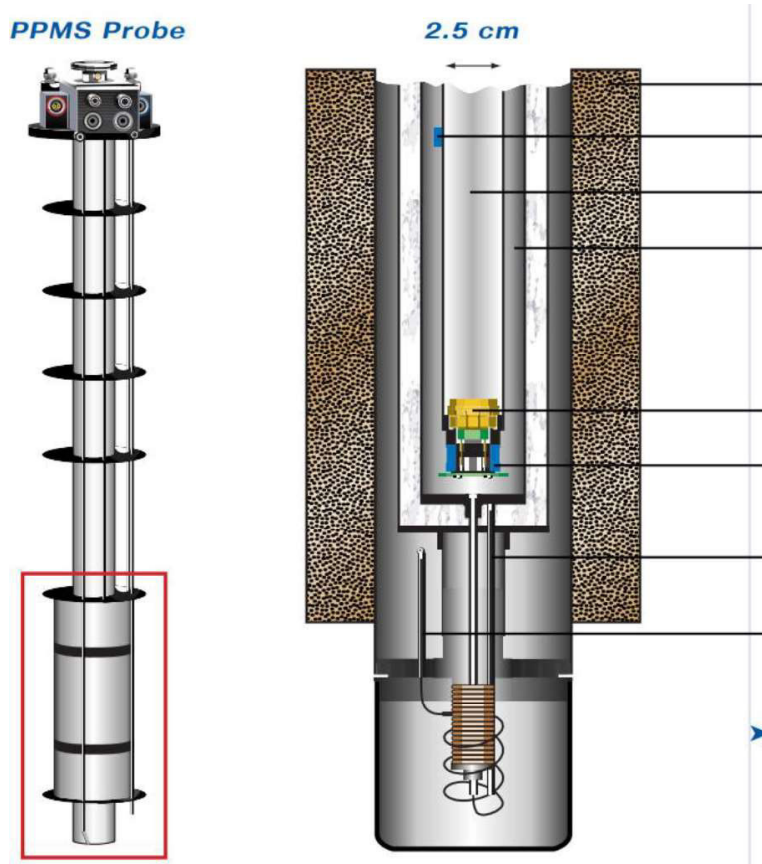

Magnet

Thermometer

Sealed sample space

Cooling annulus

Puck

Heaters and thermometers (2)

Dual impedance system

Multiple thermometers accurately monitor while heaters quickly control the system temperature.

Figure 2.12: Design of the Physical Properties Measurement System. Sample is mounted on a sample holder (puck) located in the PPMS probe. Located in the center of a superconducting magnet coil and a liquid helium cryostat electrical measurements can be conducted at variable temperatures and B-fields. Taken from [37].

Moreover, we used the van der Pauw-method which allows to determine the resistance in an arbitrarily shaped sample.[186] Figure 2.13 shows the sample geometry as used in the experiments. Here, the van der Pauw-resistance can be defined as $R_{2134}=V_{34} / I_{21}$. Then, one can derive the conductivity of the sample to be [186]

$$
\sigma=\frac{\ln (2)}{\pi} \cdot \frac{2}{R_{2134}+R_{3241}}[f(Q)]^{-1} \quad \text { with } \quad Q=\frac{R_{2134}}{R_{3241}}
$$

$Q$ is here the symmetry factor and the function $f(Q)$ is correcting for a possible geometric asymmetry of the sample. For small asymmetry one can use the approximation [186] 


$$
f \approx 1-0.346657 \cdot A-0.09236 \cdot A^{2} \quad \text { with } \quad A=\left[\frac{Q-1}{Q+1}\right]^{2}
$$

In addition, the result improves by averaging over several combinations of the contact geometry sketched in Figure 2.13. Gold contacts have been brought onto the graphene sample by a simple shadow mask procedure and are subsequently bonded to a sample holder via aluminum wires.

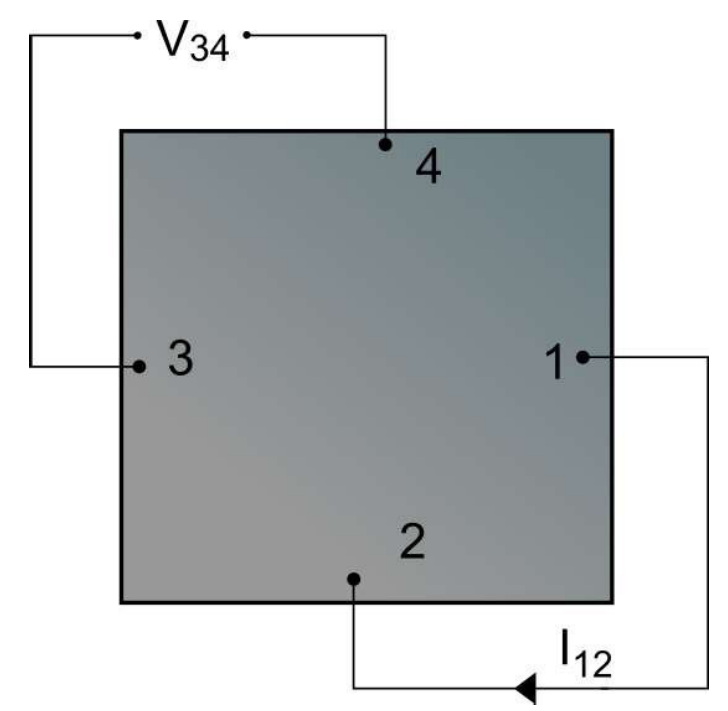

Figure 2.13: Geometry of van der Pauw resistance measurements. The sample is contacted in 4-point geometry. While a current $I_{12}$ is applied to the contacts 1 and 2 the voltage drop $V_{34}$ is measured along contacts 3 and 4 . 


\section{Transport properties of localized defects in graphene investigated by scanning probe methods}

\subsection{Introduction}

In this chapter, we use scanning tunneling potentiometry (STP) and Kelvin probe force microscopy (KPFM) to study 1D defects in graphene. These are in particular atomic steps, monolayer/bilayer graphene-interfaces, wrinkles and grain boundaries (see also Figure 2.2).

Prior to this work defects in graphene on $\mathrm{SiC}$ have been studied already along with the local sheet conductivity of the monolayer and bilayer areas by scanning tunneling potentiometry [29, 30, 42, 80], conductive AFM [57] and in transport resistance measurements [28]. Ji et al. first demonstrated that the presence of SiCsubstrate steps and monolayer-bilayer interfaces cause a voltage drop in the electrochemical potential as shown in Figure 3.1. Analyzing the magnitude of the voltage drop they could deduce values for the defect resistance $\rho_{\text {Defect }}{ }^{16}$ as well
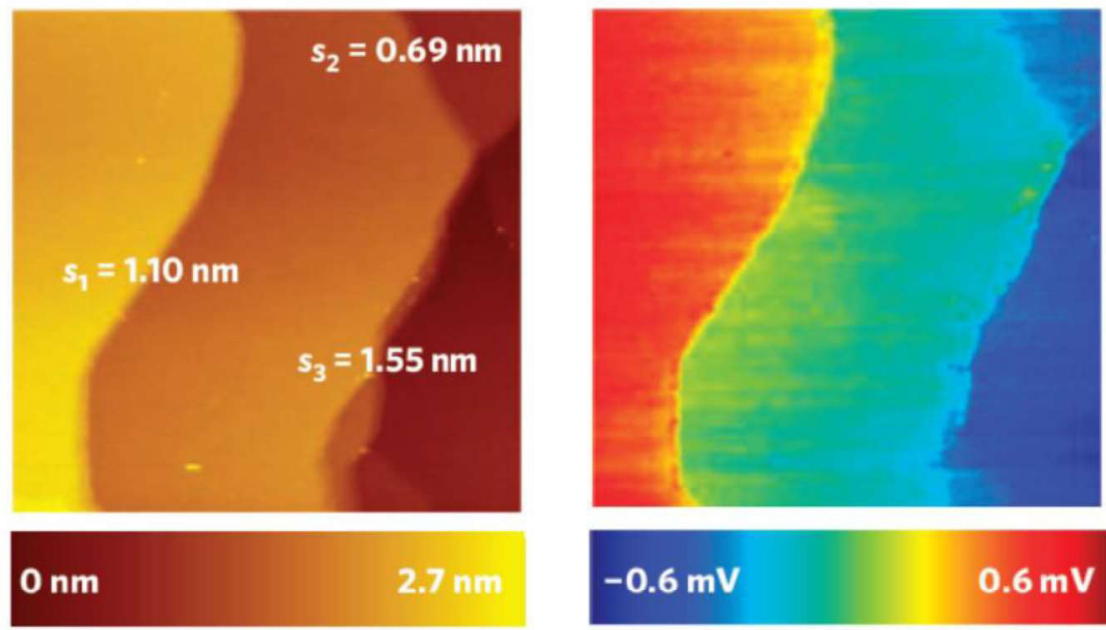

Figure 3.1: Scanning Tunneling Potentiometry of SiC-graphene. The steps and interfaces shown in the STM topography on the left cause sharp jumps in the potential shown on the right. The monolayer and bilayer graphene sheets show a linear voltage drop. Reprinted (adapted) with permission from Ref. [80]. Copyright (2012) Nature Publishing Group].

16 The values they obtained are summarized in Table 5.2 in the discussion in chapter 5. 
as models for the underlying scattering mechanism. A local change in doping and wavefunction mismatch have been proposed to cause the dominating scattering for SiC-substrate steps and monolayer(ML)-bilayer(BL) interfaces, respectively $[57,80,108]$.

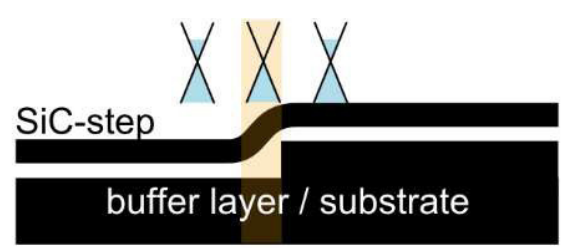

For a SiC-substrate step, the monolayer graphene sheet was found to be continuously crossing the defect $[80,100]$ (see also Figure 2.2). Therefore, Ji et al. suggested that the origin of the defect resistance stems from the curvature at the step. They speculated that this bending of the sheet might lead to $\sigma-\pi$ hybridization [80]. In a theoretical work by Low et al., the authors found scattering due to the curvature of graphene to be rather small [108]. On the other hand they suggested that the strong $n$-doping in graphene on $\mathrm{SiC}(0001)$ induced by the substrate $[100$, 137] (see section 2.1.1) is reduced in the

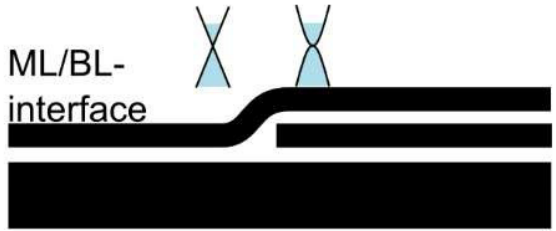

Figure 3.2: Scattering mechanisms at structural defects in SiC-graphene. For a SiC-step the graphene is detached from the underlying substrate leading to a change in doping. Thus, a nin-junction is induced. Due to the difference in energy dispersion, the additional electronic transition at the ML/BL-interface leads to a higher resistance. vicinity of the step due to detachment. This is sketched in Figure 3.2a. It effectively induces a nin-junction. Experimentally, the defect resistance was found to be roughly linearly increasing with step height $[57,80]$, which was explained with a longer intrinsic doping region [108].

Concerning the ML/BL-interface, Ji et al. suggested that the larger defect resistance compared to substrate steps is due to the change in electronic structure (See Figure 3.2b, also section 1.1) leading to a wave function mismatch [80]. Whereas the bilayer wave functions have a large amplitude on both layers the difference in $\mathrm{ML}$ and $\mathrm{BL}$ wave functions needs to be matched at the interface. This requires intermixing of wave functions from different bands in the bilayer. This is supported by first-principle calculations by Giannazzo et al., who found a particular weak coupling between the monolayer's $\pi$-bands and the lower band of the bilayer [57]. Theoretically, Nakanishi et al. found an exponentially decaying evanescent wave in the bilayer to be crucial for the connection at the interface [126].

Within the scope of this thesis, we extended these existing studies in two ways. First, in section 3.2 we analyze defects in SiC-graphene with very high spatial resolution in the sub-nanometer range. This enables us not only to discuss the magnitude, but also the position of the voltage drop, which can in case of a monolayer-bilayer junction reach up to several nanometers into the bilayer. This 
allows us to improve the models of the underlying scattering mechanism. We show that the spatial evolution can be described in a semi-classical model due to the interlayer coupling between the two layers in bilayer graphene which effictively creates a contact resistance problem.

Second, in section 3.4 we combine our STP setup with an additional magnetic field. This kind of experiment has not been conducted up to now. Thus, we are able to study for the first time the voltage drop of atomic-scale defects as a function of an external parameter: the magnetic field. Here, we find that the defects behave inherently different than the pristine monolayer and bilayer graphene areas and that the magnitude of the voltage drop does not change for defects in the presence of a magnetic field; a consequence of the different scattering mechanism as discussed above. Moreover, though we conducted our measurements at low temperatures $(6 \mathrm{~K})$ we still find good agreement of $\rho_{\text {Defect }}$ with studies conducted at much higher temperatures $[30,80]$. Therefore, we find strong evidence that the defect resistances of the defects discussed above are also independent of temperature.

In contrast to graphene on SiC, CVD-graphene grown on polycrystalline copperfoil shows different types of defects stemming from the non-epitaxial growth and the transfer to $\mathrm{SiO}_{2}$ (see subsection 2.1.2). These scattering centers have been analyzed previously due to their possible limitations to device performance, in particular grain boundaries [44, 76, 94, 171, 181, 210] and wrinkles (local folds in the graphene sheet) $[30,208,220]$. While for grain boundaries a variety of different results of $\rho_{\text {Defect }}$ can be found in literature ranging from $8-43000 \Omega \mu \mathrm{m}$, the values obtained for wrinkles so far are strongly limited to a single wrinkle resistance $(200 \Omega \mu \mathrm{m})$ measured experimentally by STP [30]. ${ }^{17}$ Theoretically, Zhu et al. predicted a resistance of $200 \pm 150 \Omega \mu \mathrm{m}$ for a folded wrinkle. By using the non-equilibrium Green's function (NEGF) method they found interlayer coupling in collapsed and folded wrinkles (see Figure 3.3a) to be the dominant scattering mechanism. Their results are shown in Figure 3.3b and are independent of the height of the standing wrinkle. This independence can be explained with the interlayer tunneling from one side of the wrinkle to the other shown in Figure 3.3c. In contrast, they were not able to determine the resistance across such a wrinkle in transport experiments [220]. Yan et al. studied wrinkles using KPFM in functionalized graphene sheets and found no significant contribution [208].

In the study presented in section 3.3 we use the technique of KPFM with an additional cross voltage to investigate defects in graphene on $\mathrm{SiO}_{2}$. This technique has been in similar ways already used to study transport in a wide range of sample

17 See also Table 5.1 in chapter 5 for an overview. 
systems including graphene [140, 211], functionalized graphene [208], Si nanowires [4], carbon nanotubes [139] and quantum dot field effect transistors [214]. By improvements in the setup and especially the data evaluation discussed in section 3.3, we are able to obtain large area 2D-transport maps without the additional influence of work function differences that is usually superimposed on the signal [see Eq. (2.21)]. This allowed us for the first time to evaluate the defect resistance of structural defects in graphene on $\mathrm{SiO}_{2}$ with KPFM. Besides the different substrate and technique, this study differs from the other works in this chapter by being conducted at room temperature and in ambient conditions. We obtain the following results. First, we could limit the influence of grain boundaries to $<80 \Omega \mu \mathrm{m}$. Second, we additionally determine defect resistances for wrinkles (folded wrinkles) of $30-$ $50 \Omega \mu \mathrm{m}(50-80 \Omega \mu \mathrm{m})$. We find evidence for a temperature-independent defect resistance of folded wrinkles compatible with interlayer transport as the dominating scattering mechanism discussed theoretically by Zhu et al. [220]. We additionally find from work function measurements that the electronic structure of folded wrinkles resembles a trilayer graphene system (As can be seen in Figure 3.3a, right). This would link the increased resistance in trilayer-like collapsed wrinkles to that of $\mathrm{ML} / \mathrm{BL}$-interfaces discussed above, where we also found the increase in defect resistance due to interlayer coupling to be dominant.
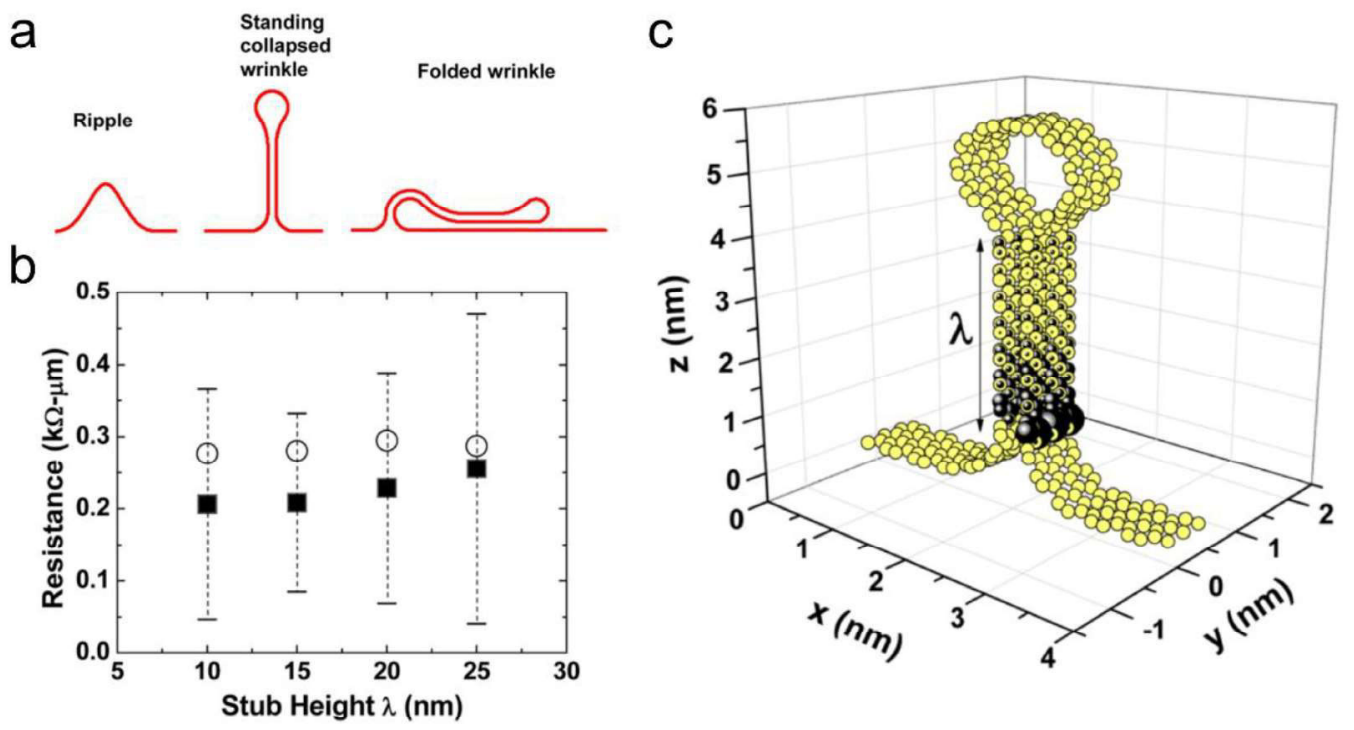

Figure 3.3: Quantum transport modeling across a collapsed wrinkle. (a) Schematic of different classes of graphene wrinkles observed by Zhu et al. [220]. (b) Defect resistance of a collapsed graphene wrinkle of different height $\lambda$. [Open circles: top of the wrinkle cut open leading to pure interlayer transport; filled squares: closed wrinkle as shown in (c)] (c) Simulated wrinkle structure with black bubbles indicating out-of-plane current density. Here, the bubble radius is proportional to the magnitude of the current density. Reprinted (adapted) with permission from Ref. [220]. Copyright (2013) American Chemical Society. 
3.2 Spatial extent of a Landauer residual-resistivity dipole in graphene quantified by scanning tunnelling potentiometry

\title{
3.2 Spatial extent of a Landauer residual-resistivity di- pole in graphene quantified by scanning tunnelling potentiometry
}

\author{
P. Willke, T. Druga, R. G. Ulbrich, M. A. Schneider and M. Wenderoth
}

Nature Communications 6: 6399, March 2015

DOI: $10.1038 /$ ncomms7399

Electronic transport on a macroscopic scale is described by spatially averaged electric fields and scattering processes summarized in a reduced electron mobility. That this does not capture electronic transport on the atomic scale was realized by Landauer long ago. Local and non-local scattering processes need to be considered separately, the former leading to a voltage drop localized at a defect, the so-called Landauer residual-resistivity dipole. Lacking precise experimental data on the atomic scale, the spatial extent of the voltage drop remained an open question. Here, we provide an experimental study showing that the voltage drop at a monolayer-bilayer boundary in graphene clearly extends spatially up to a few nanometers into the bilayer and hence is not located strictly at the structural defect. Moreover, different scattering mechanisms can be disentangled. The matching of wave functions at either side of the junction is identified as the dominant process, a situation similar to that encountered when a molecule bridges two contacts.

\subsubsection{Introduction}

The description of electron transport requires paradigms bridging all length scales from atomic to sample dimensions. As a result, most conductivity measurements include a variety of very different scattering mechanisms that could typically be disentangled only by theory. Moreover, most of our electronic devices today are characterized by a global conductivity or mobility of carriers which are often described with semiclassical concepts of transport, for example, the Boltzmann equation. That electron propagation through a sample has to be described by quantum mechanics becomes apparent only in cleverly designed experiments, for example, as universal conduction fluctuations, conductance quantization and the quantum Hall effect [31]. These experiments and the corresponding theoretical description constitute the wealth of collected knowledge on the topic mesoscopic transport. In contrast, non-equilibrium phenomena of electronic transport on the atomic scale are less well explored experimentally. Such measurements have been carried out at two-dimensional conductors [21, 71] graphene being a recent prominent system $[29,30,57,80,192]$. The experiments demonstrated the influence of non-local 
scattering processes (for example, electron-phonon or electron-electron scattering) in defect-free sample regions as well as electron scattering at localized defects like steps, interfaces and grain boundaries. Scattering at these defects was up to now only observed as localized discontinuities in the voltage drop, that is, experiments did not resolve the shape of the potential across a localized scatterer. The answer to the simple question, 'Where does the voltage drop occur?' is of specific interest as it gives access to the inherently non-equilibrium quantum mechanical processes.

In the following, we show that the voltage drop at a monolayer-bilayer (ML-BL) interface in graphene extends several nanometers into the bilayer. This is in contrast to the situation at substrate steps covered with $\mathrm{ML}$ graphene and in contrast to previous reports. We conclude that this effect is caused by the electronic coupling within the transition region to the electron reservoir of the $M L$ or $B L$ side. In this sense, the transition region bridges two electron reservoirs similar to a molecule in transport across molecular break junctions [132, 207]. We further demonstrate that different scattering mechanisms can be distinguished revealing the rather small influence of the detachment of the graphene layer from the substrate step.

\subsubsection{Results}

\subsubsection{The monolayer-bilayer interface in epitaxial graphene}

Using scanning tunnelling potentiometry (STP) [125] at $6 \mathrm{~K}$ (see Figure 3.4a), we demonstrate that the spatial characteristics of the voltage drop can be resolved with sub-nanometer resolution. At the atomic scale, a current flowing across a structural defect leads to the accumulation of electrons on one and depletion on the other side. This Landauer residual-resistivity dipole [99] is caused by the reduced transmission probability of the electrons past a defect. Consequently, the electrochemical potential (ECP) changes locally leading to the observed voltage drop [15, 16, 27, 31, 124]. In the case of one-dimensional defects in a two-dimensional conductor, an interesting parallel can be drawn to electron transport through single molecules [132], which has been theoretically studied in great detail in the past. According to Xue and Ratner [207], the change in the ECP occurs over the length of the molecule since different molecular orbitals couple differently to the electron reservoirs at the end of the molecule. The ML-BL junction in graphene [136] on silicon carbide ( $\mathrm{SiC}$ ) serves as an ideal system to study the shape of the local ECP involving several competing scattering mechanisms under discussion $[57,80,108]$. The atomic scale structure encountered at such a ML-BL junction is depicted in Figure 3.4b. A graphene sheet from the ML side starts to coat an underlying additional graphene sheet on the $\mathrm{BL}$ side. This configuration is known to 
be caused by the thermal decomposition of $\mathrm{SiC}$ during growth of graphene, which always leads to new graphene layers under preceding sheets [100,134]. For the

a

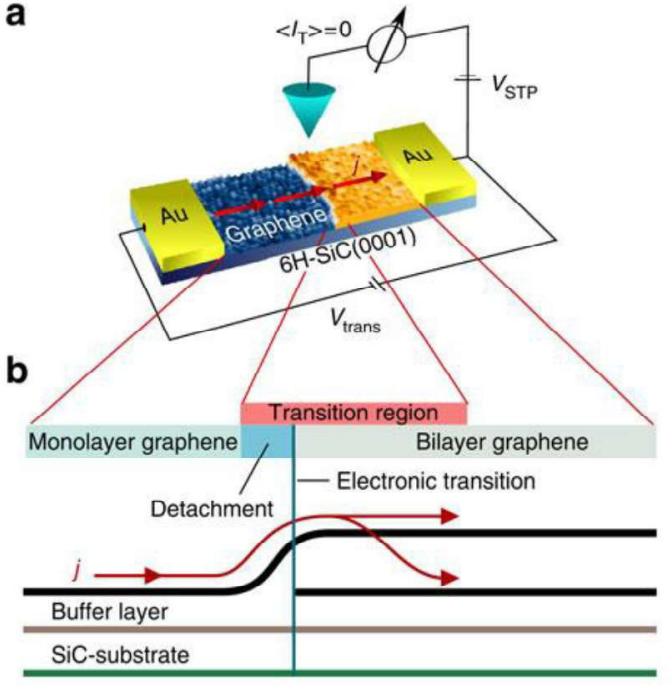

c



d

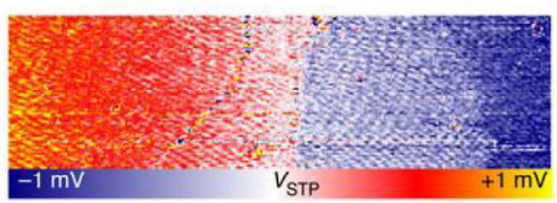

e

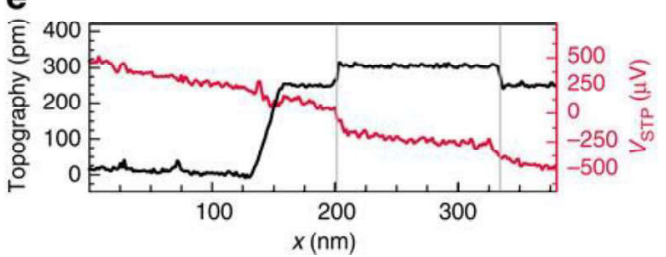

Figure 3.4: Scanning tunnelling potentiometry and the graphene monolayer-bilayer junction. (a) Schematic of the setup of our scanning tunnelling potentiometry experiment. The epitaxially grown graphene sample is contacted with gold in two-point geometry. The voltage $\left.V_{\text {STP }}(x, y)\right|_{I_{T}=0}$ necessary to compensate a net tunnel current $I_{T}$ is recorded and mapped. It represents the voltage drop along the sample induced by the voltage $V_{\text {Trans. }} j$ is the current density in the sample. (b) Sketch of the structural transition from monolayer $(\mathrm{ML})$ into bilayer $(\mathrm{BL})$ graphene and the consequences for electron transport. The upper graphene layer is continuous and connects to the lower bilayer sheet via interlayer coupling. The electrical resistance of the junction is caused by the detachment of the graphene sheet from the underlying substrate and the transition of the electronic states from monolayer- to bilayer-like. Although the former leads to a voltage drop directly at the step, our experiments show that the transition of the electrons into the bilayer happens on a nanometer scale. (c) Large scale topography and (d) the respective potential map for $V_{\text {STP. }}$ (e) Section along the white line in $\mathbf{c}$ that reveals the delocalized linear voltage drop on the monolayer and bilayer sheets, whereas a distinct localized voltage drop is found at the ML/BLinterfaces (marked with grey lines) and a less prominent one at the substrate step covered with a ML graphene sheet. (Imaging conditions: $\mathrm{I}_{\mathrm{T}}=0.2 \mathrm{nA}, \mathrm{V}_{\text {Bias }}=-30 \mathrm{mV}, \mathrm{j}=12 \mathrm{Am}^{-1}$ ).

$\mathrm{ML}-\mathrm{BL}$ junction, a change in electronic properties occurs directly at the structural boundary (blue vertical line in Figure 3.4b). It has been proposed that the wave function mismatch at the junction leads to a reduced transmission probability of ML states into the $B L$ region [30, 57, 80]. A highly localized jump of the ECP in ref. [57] is explicitly attributed to this dominant scattering mechanism at a ML-BL boundary. Moreover, at the junction, the continuous upper sheet is bent and detached from the substrate (light blue area in Figure 3.4b). A similar configuration is encountered when a $\mathrm{ML}$ graphene sheet covers a substrate step (ML-ML junction). In this case, the detachment of the graphene sheet and its consequently changed local electron density was theoretically found to be the dominating contribution to the resistance [108]. 


\subsubsection{Large scale STP}

In Figure 3.4c, the topography of a typical structure of our epitaxial graphene samples is shown. The simultaneously acquired potential map in Figure 3.4d shows the voltage drop on a scale of several $100 \mathrm{~nm}$ caused by a current density in the graphene layer of $j=12 \mathrm{Am}^{-1}$. (For evaluation of low-temperature STP data, see Supplementary Note 1 [subsection 3.2.7.3] and Supplementary Figure 3.4). It reflects the scattering mechanisms discussed above. A section along the white dashed line of Figure 3.4c of both topography and potential map shown in Figure $3.4 \mathrm{e}$ reveals the large voltage drop associated with the ML/BL interface. In the following, we will resolve the transition of the ECP on the atomic scale.

a
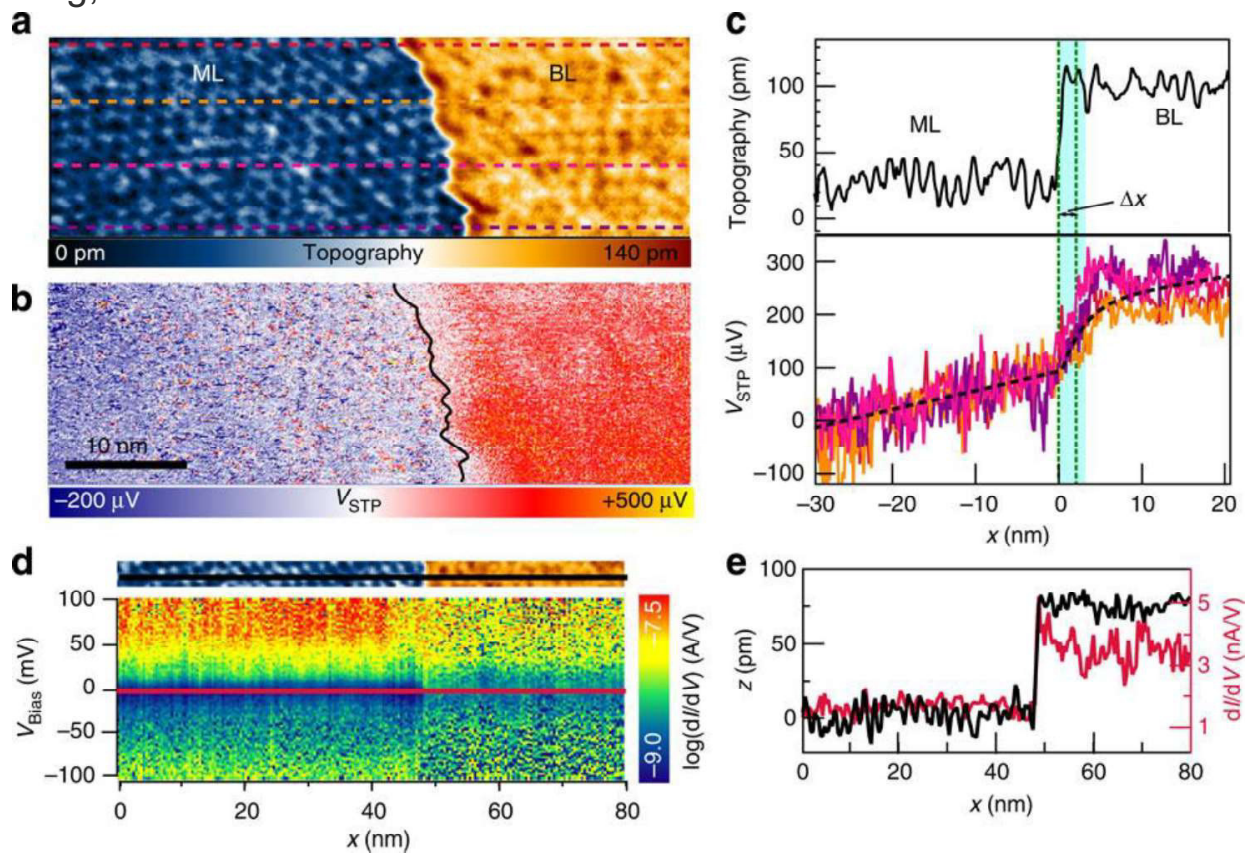

Figure 3.5: High-resolution potentiometry at a ML/BL junction. (a) Topography (imaging conditions: $I_{T}=0.2 \mathrm{nA}, V_{\text {Bias }}=-50 \mathrm{mV}, j=10 \mathrm{Am}^{-1}$ ) and (b) corresponding potential map for $V_{\text {sтP. }}$. The black line indicates the position of the step in (a). (c) Data sections comparing the topography and voltage drop along the lines in a. All sections (average of 10 lines) have been shifted laterally, so that the corresponding step is located at $x=0 \mathrm{~nm}$ for all sections. The green dotted lines indicate the lateral offset $\Delta x$ between the FWHM value points of the topography and the potential for the pink curve. The blue-shaded area indicates the distance to the maximum of the voltage drop. The drop is shifted into the bilayer region with a smooth evolution of the potential, which is varying on a local scale (see purple and yellow section). The black dotted line shows the fit of the voltage drop to the classical model as described in the text. (d) Scanning tunnelling spectroscopy (STS) measurement crossing the ML/BL junction in (a) as indicated at the top. (e) Section along the black and red lines in (d) comparing the topography with the spectroscopy at $0 \mathrm{mV}$. The transition in the STS is strictly located at the topographic position of the step (imaging conditions: $\mathrm{I}_{\mathrm{T}}=0.35 \mathrm{nA}, \mathrm{V}_{\text {Bias }}=-100 \mathrm{mV}$ ). 
3.2 Spatial extent of a Landauer residual-resistivity dipole in graphene quantified by scanning tunnelling potentiometry

\subsubsection{Extent and shift of the electrochemical potential}

In Figure 3.5, a topography map (Figure 3.5a, Supplementary Figure 3.9) and the corresponding potential map (Figure $3.5 b$ ) that focus on a ML/BL step with higher resolution is shown. In the potential map, we find a distinct lateral extension of the $M L$ potential into the BL with respect to the localized topographic step (black line). The onset of this voltage drop is located at the topographic step while its final value is found several nanometers inside the bilayer. This becomes even more evident in Figure 3.5c, where we show sections along the colored lines indicated in Figure 3.5a. The difference between the midpoint of the step in the topography and the end of the potential is indicated for the pink line by the blue-shaded area, which is found to be $\sim 3 \mathrm{~nm}$ wide. This difference holds also for the other sections or becomes even larger (purple section). Up to its maximum, the potential rises continuously. The smooth potential slope and its spatial extent are not expected from previous models for the ML/BL interface that predict the potential drop directly at the electronic transition [57]. To prove that this electronic transition between ML and $\mathrm{BL}$ is located at the topographic step, we have carried out a spectroscopy measurement shown in Figure 3.5d. In the range of $\pm 100 \mathrm{mV}$, it shows the differential conductance for $\mathrm{n}$-doped SiC-graphene for both $\mathrm{ML}$ and $\mathrm{BL}$ graphene consistent with others [108]. Within the experimental resolution of $0.4 \mathrm{~nm}$, topography and spectroscopy are both in perfect synchronicity at the Fermi-energy (Figure 3.5e). This shows that in contrast to the large extension of the voltage drop at the $\mathrm{ML} / \mathrm{BL}$ interface, the measured electronic spectra change abruptly at the step. Moreover, tip jumping artefacts can be excluded (Supplementary Note 2 [subsection 3.2.7.4], Supplementary Figure 3.10).

In total, we evaluated the voltage drop at 13 independent $M L-B L$ boundaries in a quantitative manner including configurations with and without an underlying substrate step. As a quantitative measure (Supplementary Note 3 [subsection 3.2.7.5], Supplementary Table 3.1), we use the lateral mean offset $\langle\Delta x\rangle$ between the full width at half maximum value points of the topographic and the full width at half maximum value points of the potential transition (green lines in Figure 3.5c). The result of this evaluation is shown in Figure 3.6. On average, we obtain $\langle\Delta x\rangle_{M L / B L}=+2.0 \pm 0.5 \mathrm{~nm}$. The full spatial extension of the potential drop is $\langle L\rangle_{\Delta v}=4.0 \pm 1.3 \mathrm{~nm}$ and the voltage drop is therefore almost completely located in the bilayer region. To check that this shift is particularly connected to the ML/BL interface, we analyzed substrate steps covered by a single continuous layer of graphene (ML/ML junction). In agreement with other works [30, 80], we find that the detachment of the sheet at a substrate step causes a voltage drop, but we do not observe a lateral shift leading to $\langle\Delta x\rangle_{\mathrm{ML} / \mathrm{ML}}=0.3 \pm 0.4 \mathrm{~nm}$. This shows that the extended potential shift is connected to the electronic transition between $\mathrm{ML}$ and $\mathrm{BL}$ and not to the presence of the step itself. 


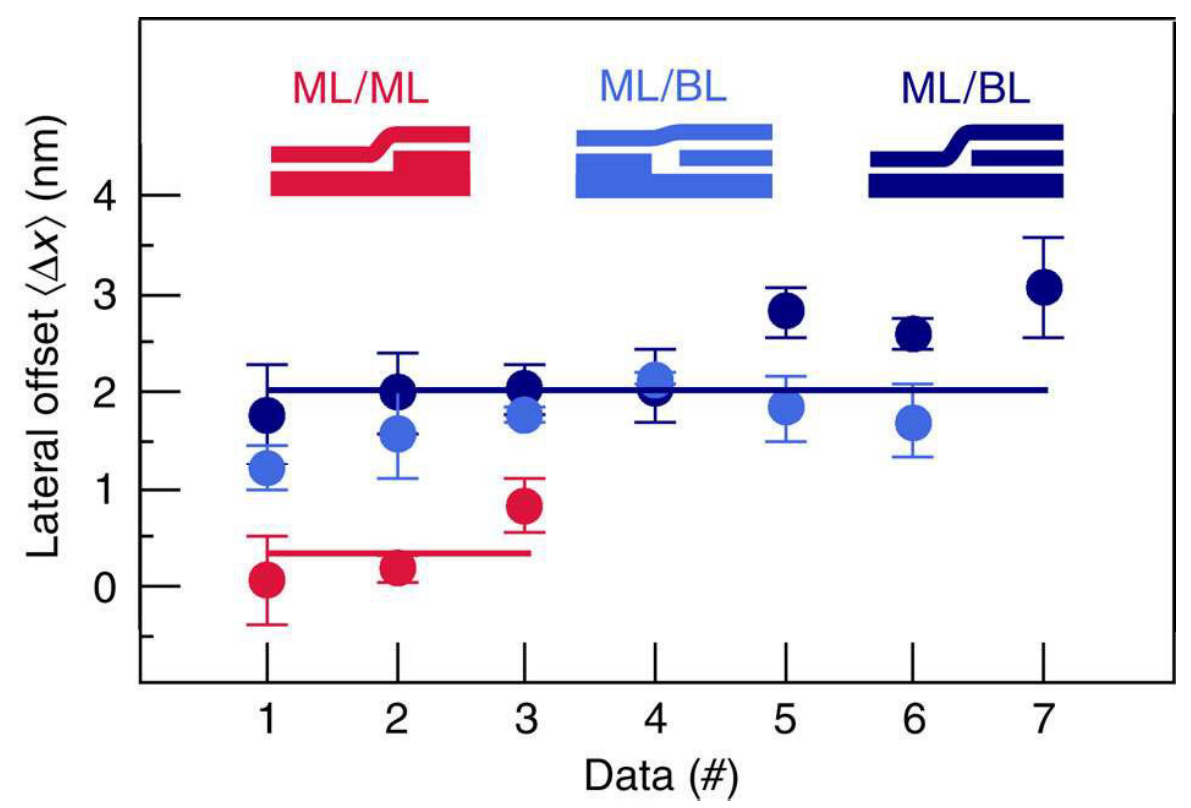

Figure 3.6: Statistic of the lateral offset. Statistic of the lateral offset $\langle\Delta x\rangle$ between the transition in the topography and the transport map. Error bars show the standard deviation. It reveals an offset into the bilayer for different types of ML/BL-steps in contrast to ML/MLsubstrate steps (red). For the ML/BL-steps, two different configurations have been observed, one involving an additional SiC-substrate step (light blue) and one without an additional step (dark blue).

\subsubsection{Separation of different scattering contribution}

Besides the electronic transition, each ML/BL junction contains also a detachment from the substrate. The additional scattering contribution should be distinguishable from the interlayer coupling at least for certain configurations of the junction. This becomes visible for the ML/BL boundary shown in Figure 3.7. Here, the lateral section of the ECP depicted in Figure 3.7c clearly reveals a two-step potential drop. First, a small drop $\Delta V_{1}$ is seen directly at the position of the step and second, a large drop $\Delta V_{2}$ occurs deep within the bilayer. We attribute $\Delta V_{1}$ to the detachment as observed for ML/ML-surface steps and $\Delta V_{2}$ to the $\mathrm{ML} / \mathrm{BL}$-interlayer coupling (Figure 3.7d) as discussed for the step in Figure 3.5.

\subsubsection{Discussion}

To explain the shift of the voltage drop at $\mathrm{ML} / \mathrm{BL}$ junctions into the bilayer side, we recall its geometry as shown in Figure $3.4 \mathrm{~b}$. The monolayer sheet is continuously connected to the upper bilayer sheet. As known from multilayer graphene and graphite, this upper bilayer sheet is connected to the lower bilayer sheet via a significantly higher interlayer resistivity compared with the in-plane resistivity [121, 170]. In addition to the higher $c$-axis resistivity, the electron density is reduced in the upper layer due to distance-dependent buffer-layer doping [137, 138] and 
3.2 Spatial extent of a Landauer residual-resistivity dipole in graphene quantified by scanning tunnelling potentiometry

therefore its in-plane resistance also is increased [136]. Theoretically, this layerdependent conductivity has been demonstrated in a gated bilayer; as for distinct voltages perpendicular to the sheet, the current in one layer can even be tuned to zero [130]. Accordingly, the difference in electron density of the second layer in combination with the high interlayer resistivity leads to a bottleneck for electron transport. The interlayer hopping process necessary to populate the lower sheet with higher electron density extends the effective transport barrier spatially into the bilayer. To model this situation, we use a resistor network, which has already been successfully applied to local transport experiments[43, 71, 80], as well as an anaIytical model derived for voltage drops at interfaces in scanning probe experiments[193] (Supplementary Note 4 [subsection 3.2.7.6], Supplementary Figure 3.11-3.13). Both models consider the problem of electron transfer with a contact resistance, which treats the bottom sheet of the bilayer as a contact to the top one. The best fit with this model is shown by the black dotted line in Figure 3.5c. Though we only apply a classical model neglecting quantum coherence, the results reproduce the observed shape and length scale of the ECP. Moreover, the model allows to extract a contact resistance of $R_{c}=4.2 \times 10^{-10} \Omega \mathrm{cm}^{2}$ which is reasonable when compared with $c$-axis resistivity in graphene and graphite (Supplementary Note 4 [subsection 3.2.7.6]).
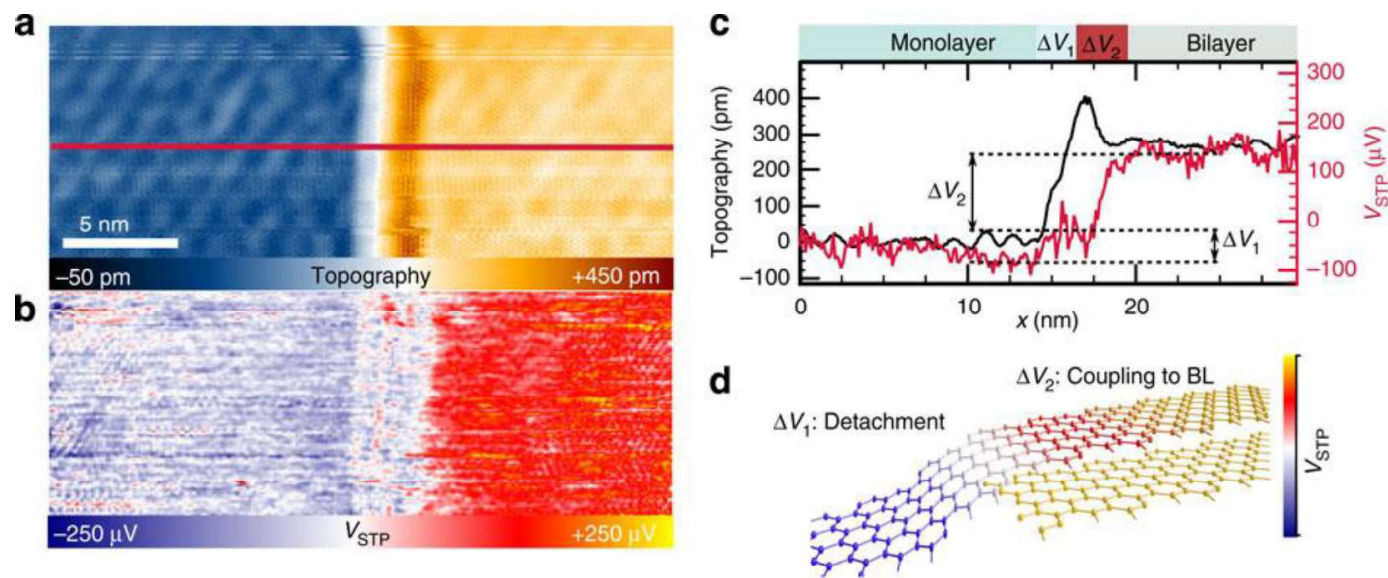

Figure 3.7: Resolving different scattering components. (a) Topography of a ML/BL boundary and (b) the corresponding change in the ECP V STP (imaging conditions: $l_{T}=0.24 \mathrm{nA}, V_{\text {Bias }}=-40 \mathrm{mV}, j=4 \mathrm{Am}^{-1}$ ). (c) Sections along the line in (a). Two distinct voltage drops, $\Delta V_{1}$ directly at the step and $\Delta V_{2}$ again in the bilayer are visible. (d) Scattering processes for the potential drops in (c). $\Delta V_{1}$ is caused by the detachment of the sheet from the substrate reducing the doping. The major contribution $\Delta V_{2}$ is again shifted into the bilayer and results from the interlayer coupling.

A comprehensive explanation for the extension of the ECP into the BL side should include the decaying states present on the bilayer according to Nakanishi et al.[126] This weak coupling determines the local ECP in the same way as was proposed by theory for single molecules. Here, a reduction in orbital overlap leads 
to an effective potential barrier for electron transport inside a molecule and at its contacts $[132,207]$. The shift of the potential drop into the bilayer indicates a better coupling of the junction region to the monolayer than to the bilayer side. This picture of electron transport also becomes important when graphene is considered as an electrode material in molecular junctions [107].

The results obtained from Figure 3.7 not only show that the transition region contains more than one scattering mechanism, but also that their relative magnitude can be resolved by STP on the scale of few nanometers. Moreover, in the region between the two potential drops, the potential does not change significantly (Figure 3.7c). After the first increase in potential $\Delta V_{1}$ due to the detachment of the graphene, it takes a certain distance before the onset of $\Delta V_{2}$. Since in the topography the wrinkle structure is located in this region, we hypothesize that the bending of the upper sheet as well as other mechanisms located in this region (for example, interface states) are less relevant than the electronic $\mathrm{ML} / \mathrm{BL}$ transition. This would fit to theoretical results showing that bending has a negligible effect on the resistance of graphene [108].

Up to now highly spatially resolved information of a transition region of the electrochemical potential in the presence of a localized barrier was accessible only by theoretical treatments[15, 16, 27, 31, 99, 124, 132, 207]. Dissecting experimentally, the spatial evolution of the ECP with Angstrom resolution at low temperature opens a new way to non-thermal equilibrium, molecular and coherent quantum transport phenomena. This includes conceptually open questions like the transition from a quantum mechanical description to diffusive scattering.

\subsubsection{Methods}

\subsubsection{Sample preparation}

Samples with epitaxial mono- and bilayer graphene are prepared by thermal decomposition of $\mathrm{n}$-doped $6 \mathrm{H}-\mathrm{SiC}(0001)[155]$ at $T=1400-1600^{\circ} \mathrm{C}$ under ultra-high vacuum (UHV, $10^{-10} \mathrm{mbar}$ ). The samples $(2 \mathrm{~mm} \times 7 \mathrm{~mm}$ ) are electrically contacted ex situ with gold contacts of $100 \mathrm{~nm}$ thickness by thermal evaporation through a shadow mask. After reinsertion into the UHV chamber, the samples are heated up to $350^{\circ} \mathrm{C}$ for $30 \mathrm{~min}$ to eliminate surface contaminations before they are transferred in situ to a homebuilt low-temperature scanning tunnelling microscope. All the measurements were performed at $6 \mathrm{~K}$ sample temperature.

\subsubsection{Scanning probe measurements}

STP measurements are taken at every image point by adjusting the ECP at the tip at fixed tip-sample distance. For STP, the applied bias voltage is switched off while only the transport potential across the sample remains. The potential at the tip is 
3.2 Spatial extent of a Landauer residual-resistivity dipole in graphene quantified by scanning tunnelling potentiometry

adjusted in a way that the tunnelling current $l_{T}=0$. Subsequently, the voltage $\left.V_{\mathrm{STP}}(x, y)\right|_{I_{\mathrm{T}}=0}$ necessary to compensate the net tunnel current is recorded (See Figure 3.4a). This voltage $V_{\mathrm{STP}}=\mu_{E C P} / e$ has been referred to as the local ECP, which is here inherently defined by the STP method $[41,71,193]$. The measurements are made at different values of the electron current in the sample plane, especially at zero and forward and reversed current as defined by the potential applied to the sample contacts. The details of our specific setup are published elsewhere [41].

\subsubsection{Acknowledgement}

This work was supported by the Deutsche Forschungsgemeinschaft (DFG) priority program 1459 Graphene.

\subsubsection{Author contributions}

T.D., M.W. and R.G.U. planned the experiments; P.W. and T.D. contributed equally to this work; they carried out the experiments and the data analysis. P.W., T.D., M.W. and M.A.S wrote the manuscript; and all the authors discussed the results and commented on the manuscript. 


\subsubsection{Supplementary Information}

\subsubsection{Supplementary Figures}

a Topography

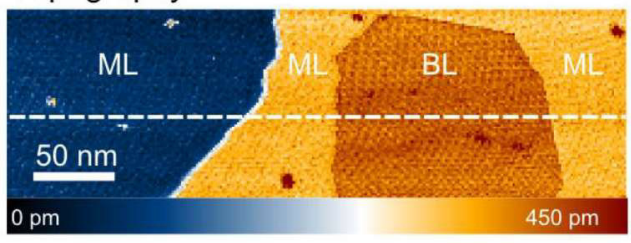

b Thermovoltage $\left(V_{\text {Trans }}=0 \mathrm{~V}\right)$

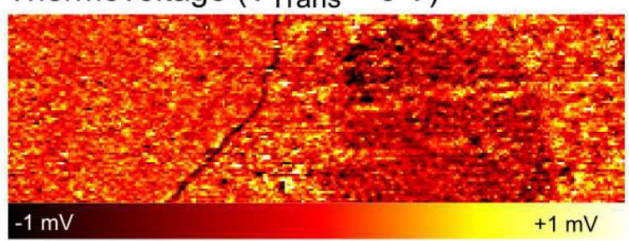

d $V_{\text {Trans }}=-10 \vee(j=-12 \mathrm{~A} / \mathrm{m})$

C $V_{\text {Trans }}=+10 \mathrm{~V}(j=+12 \mathrm{~A} / \mathrm{m})$

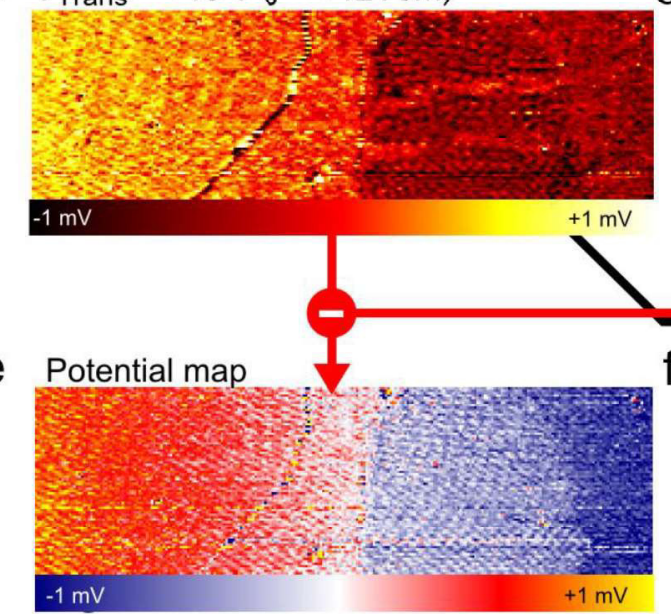

\section{$\mathrm{f}$}

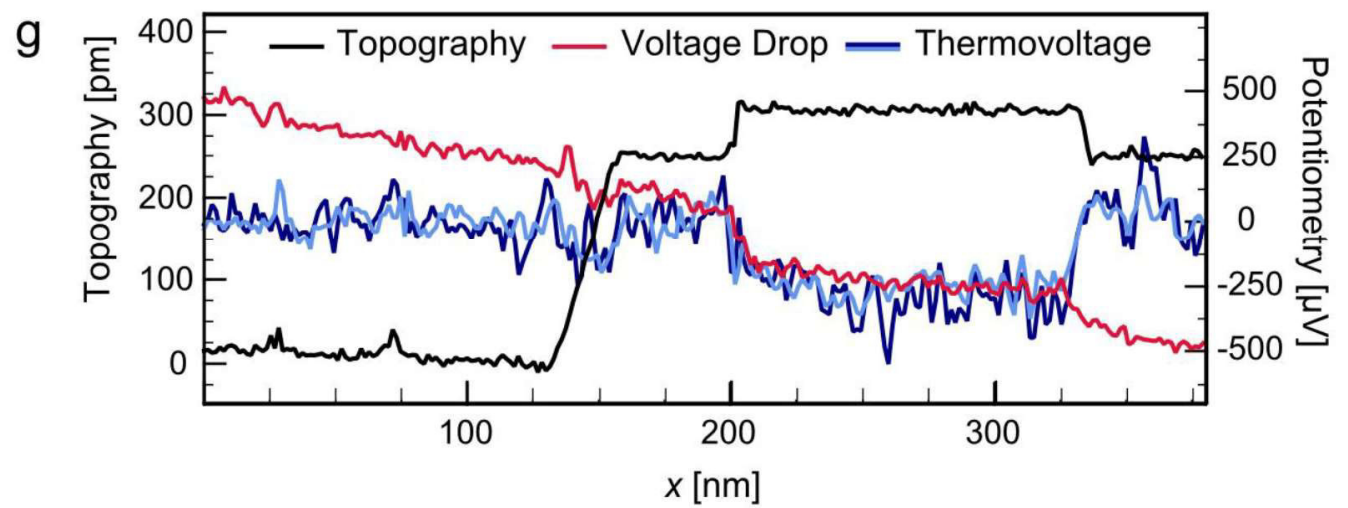

Figure 3.8: Evaluation of the STP measurements. (a) Topography of a sample region containing an area of monolayer (ML) graphene separated by a substrate step and bilayer $(\mathrm{BL})$ graphene. (b) Thermovoltage map obtained for STP-measurements with no lateral applied voltage. (c-d) STP-measurements with the indicated current density $j= \pm 12 \mathrm{Am}^{-1}$ along the two horizontal directions. (e-f) Separation of effects depending on the current direction from those independent of it. (g) Section [dashed white line in (a)] through the topography in (a), the thermovoltage in (b) (dark blue) and (f) (light blue) as well as the voltage drop in (e). Since (f) and (b) are identical, the asymmetry with respect to current direction in (c) and (d) is caused by the thermovoltage and (e) is truly representative of the voltage drop in the sample area depicted. (Imaging conditions: $I_{\mathrm{\top}}=0.2 \mathrm{nA}, V_{\mathrm{Bias}}=$ $-30 \mathrm{mV})$. 


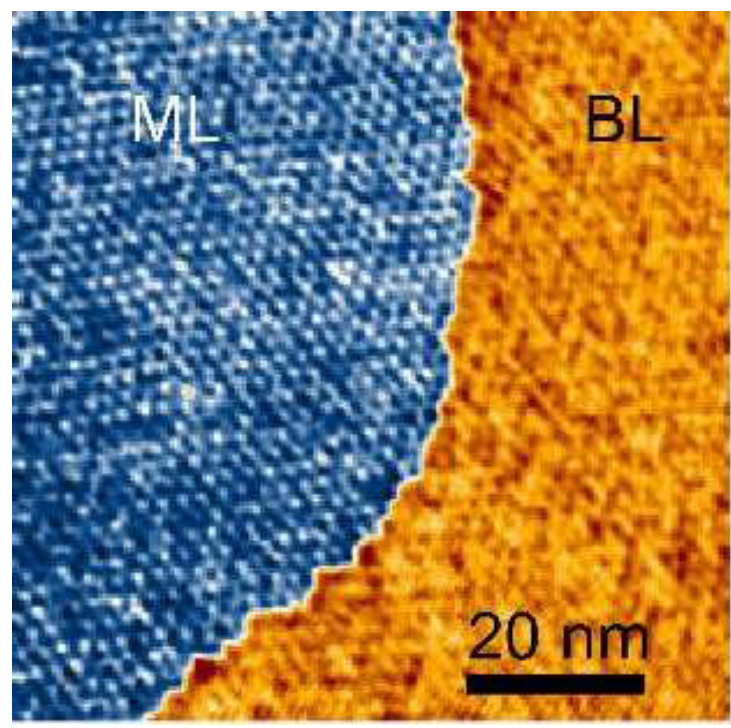

Figure 3.9: Larger topography for Figure 3.5. On the left a monolayer graphene area is shown while the respective bilayer area is on the right. The periodic lattice originates from the 6x6-superlattice known from SiC-graphene $\left(I_{T}=0.35 \mathrm{nA}, V_{\text {Bias }}=-100 \mathrm{mV}\right)$.

a

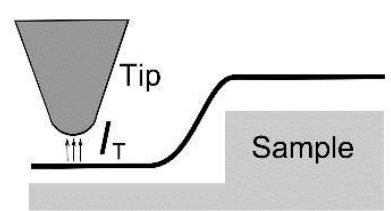

b

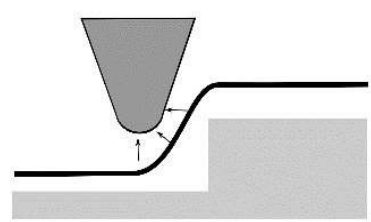

c

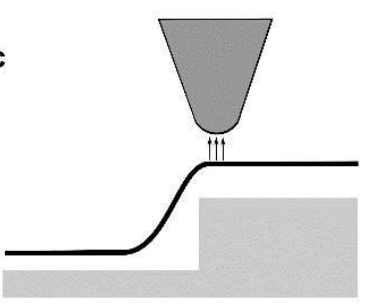

Figure 3.10: Schematic of possible tip jumping artefacts. (a) Tunneling current contribution $I_{\top}$ for a tip at the lower terrace of the step. (b) At the step, the tip receives contributions to $I_{T}$ from different locations of the step. This also alters the results of a potentiometry measurement. (c) Such artefacts are absent as soon as the tip reaches the upper terrace. 


\section{BLG}
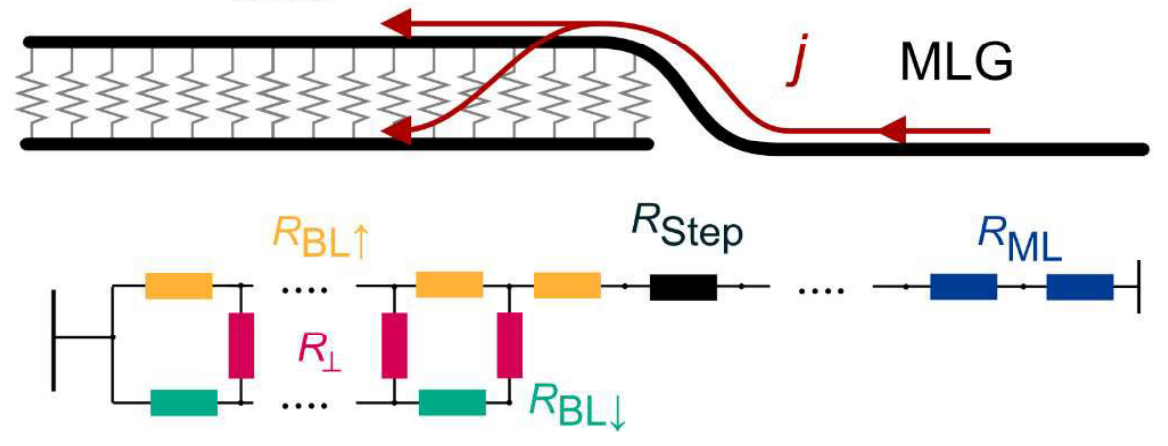

Figure 3.11: Resistor network model of the ML/BL-step. To model the shift caused by the increased interlayer resistance, we implemented a resistor network as depicted: A step resistance in the middle is surrounded by a monolayer and a bilayer side. Different resistance values for the monolayer, the step, for both layers of the bilayer and the interlayercoupling are taken into account.
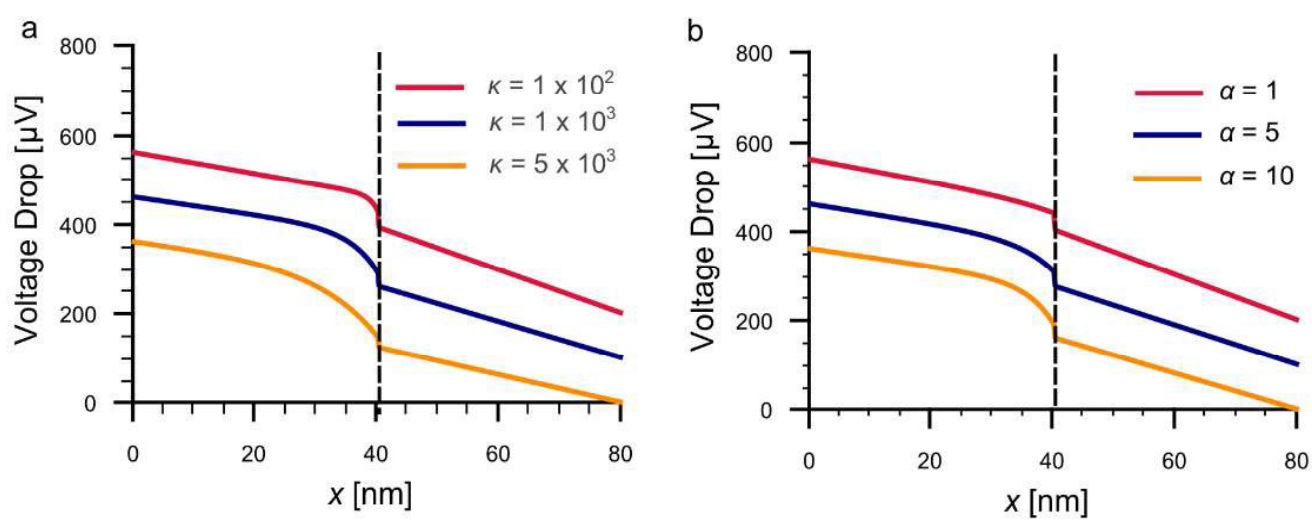

Figure 3.12: Results for the resistor network. (a) Dependence on the ratio $\mathrm{k}$ between $\mathrm{c}-$ axis and in-plane resistance. (Further parameters: $\alpha=10 ; j=10 \mathrm{Am}^{-1} ; \rho_{\mathrm{ML}}=360 \Omega$; $\rho_{\mathrm{BL}}=240 \Omega$ ) (b) Potential drop for different ratios of resistances for the upper and the lower bilayer sheet $\alpha$. (Further parameters: $\kappa=1 \cdot 10^{3} ; j=10 \mathrm{Am}^{-1} ; \rho_{\mathrm{ML}}=360 \Omega ; \rho_{\mathrm{BL}}=240 \Omega$ ) 

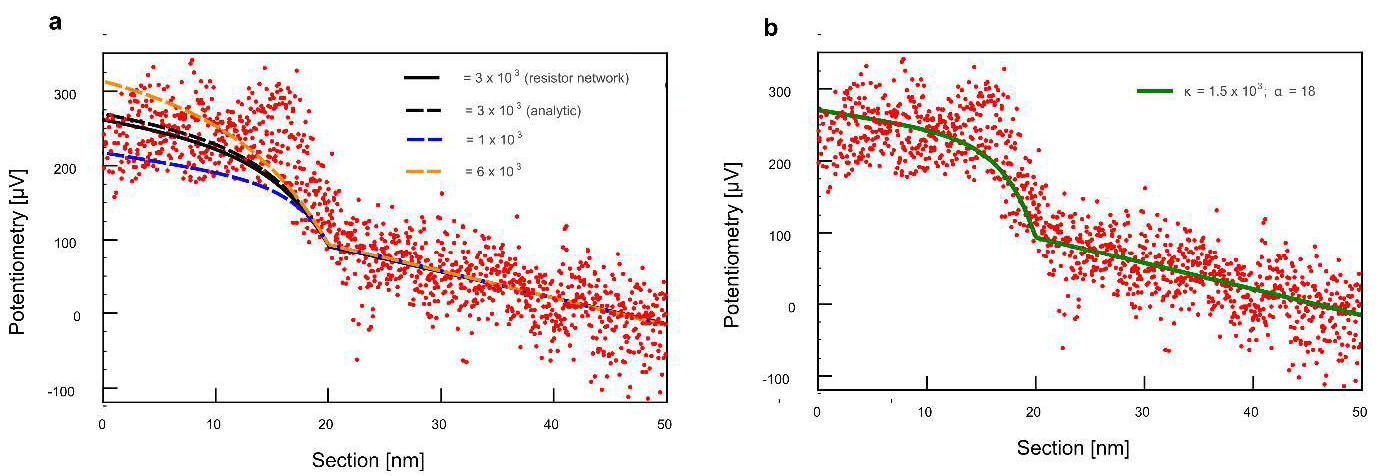

Figure 3.13: Fit of the simulation to experimental data. (a) Comparison between experimental data (red dots), the resistor network model (black solid line) and the analytical model (black dotted line) with the same parameters, both with $\kappa=3 \cdot 10^{3}$. Additionally, results for $\kappa=1 \cdot 10^{3}$ (blue dotted line) and $\kappa=6 \cdot 10^{3}$ (orange dotted line) have been plotted. (b) Fit for a changed doping factor $\alpha=18$ and $\kappa=1.5 \cdot 10^{3}$ representing the best fit we obtained within the framework of this model. (Further parameters; $j=10 \mathrm{Am}^{-1} ; \rho_{\mathrm{ML}}=360 \Omega$; $\left.\rho_{\mathrm{BL}}=240 \Omega\right)$.

\subsubsection{Supplementary Table}

$\begin{array}{lccc} & \langle\Delta x\rangle[\mathrm{nm}] & \langle L\rangle_{\Delta V}[\mathrm{~nm}] & \langle L\rangle_{\Delta z}[\mathrm{~nm}] \\ \text { ML-BL-Transition } & 2.0 \pm 0.5 & 4.0 \pm 1.3 & 0.5 \pm 0.4 \\ \text { ML-ML-Transition } & 0.3 \pm 0.4 & 2.0 \pm 1.7 & 0.9 \pm 0.5\end{array}$

Table 3.1: Averaged values for the fit parameter in the step-function model for both ML/BLand ML/ML-transition.

\subsubsection{Supplementary Note 1: Evaluation of a voltage drop at ML/BL- junctions in the presence of thermovoltage}

For low temperature STP data, the evaluation of the local voltage drop is influenced by the thermovoltage effect $[142,153]$, a thermoelectric effect due to different temperatures of tip and sample. This quantity is obtained in the same STP measurement procedure as the local ECP (See Figure 3.4a). In the following we demonstrate that it is possible to eliminate this contribution by simple mathematical operations on different voltage maps.

Tip and sample of a low-temperature STM are not necessarily at the same temperature. In this case the Seebeck-effect causes a finite current at equal ECP of tip and sample that will be canceled in the STP measurement by applying a difference between tip and sample potential of $V_{\mathrm{Th}}(x, y)$. This voltage is only related to the temperature difference but not to any transport fields. In our given setup, the sample support is held at $6 \mathrm{~K}$ while the tip is only connected via thin silver wires to 
the helium bath. As a result, we obtain local variations in thermovoltage $V_{\mathrm{Th}}$ of a few hundred $\mu \mathrm{V}$. The thermovoltage effect in STM experiments was treated by Støvneng and Lipavský[168] with the result that

$$
\left.V_{\mathrm{Th}}(x, y) \propto \frac{\pi^{2} k_{\mathrm{B}}^{2}}{6 e}\left(T_{\mathrm{T}}^{2}-T_{\mathrm{S}}^{2}\right) \frac{1}{\rho_{\mathrm{S}}(E, x, y)} \frac{\mathrm{d} \rho_{\mathrm{S}}(E, x, y)}{\mathrm{d} E}\right|_{E=E_{\mathrm{F}}}
$$

where $T_{\mathrm{T}}$ and $T_{\mathrm{S}}$ are the temperature of the tip and sample, respectively. $\rho_{\mathrm{S}}(E)$ is the local density of states of the sample. Hence, variations of $V_{\mathrm{Th}}$ at the nanometer scale stem from the lateral variation of the logarithmic derivative of $\rho_{S}(E)$ at $E=E_{\mathrm{F}}$. As a consequence the thermovoltage is sensitive to variations in the local density of states at the Fermi level comparable to scanning tunneling spectroscopy (STS) [168].

Supplementary Figure 3.8 demonstrates a large scale STP-analysis of the region shown in Figure $3.8 \mathrm{c}$ in the manuscript, which consists of a monolayer-bilayertransition and additionally a SiC-substrate step covered by graphene (ML/ML-junction). In Supplementary Figure 3.8b we plot the thermovoltage as the STP signal obtained without applying a lateral transport field. Here, a clear contrast between the monolayer and the bilayer is present due to the change in local density of states at the Fermi-energy. The maps in Supplementary Figure 3.8c and Figure 3.8d show the potentiometry measurement with applied lateral voltage of $+10 \mathrm{~V} /-10 \mathrm{~V}$. For our sample geometry, this results in a (macroscopic) current density of $j_{\text {mac }}= \pm$ $12 \mathrm{Am}^{-1}$. It is apparent that the major effect in Supplementary Figure 3.8c and Supplementary Figure $3.8 \mathrm{~d}$ is observed at the ML/BL and not at the ML/ML junction although the latter is structurally much more prominent (Supplementary Figure 3.8a).The data in Supplementary Figure 3.8c and Figure 3.8d shows asymmetries with respect to the current direction for the voltage drop at the ML/BL-interface. However, it is crucial to realize that these maps contain not only the local response to the external applied transport field, but also a contribution of the local thermovoltage (Supplementary Figure 3.8b). This prohibits a direct evaluation of the voltage drop from the data in Supplementary Figure 3.8c/Figure 3.8d. Since the thermovoltage does not depend on the current direction, its signal can be restored by averaging the data in Supplementary Figure 3.8c and Figure 3.8d whereas the true transport field can be obtained as half the difference of the two maps. This is shown in Supplementary Figure 3.8e and Figure 3.8f: The transport field in Supplementary Figure 3.8e now clearly shows distinct voltage drops across the layers and at the substrate steps whereas Supplementary Figure 3.8f only shows a contrast between $\mathrm{ML}$ and $\mathrm{BL}$ regions. The procedure, however, needs to be validated by comparing the restored thermovoltage signal with that measured in the absence of any current flow to exclude an asymmetric voltage drop with respect to current direction as has been reported recently by Clark et al. in the limit of high current densities [29]. 
The restored thermovoltage in Supplementary Figure $3.8 \mathrm{f}$ shows the same contrasts as Supplementary Figure $3.8 \mathrm{~b}$, the thermovoltage without applied lateral voltage. That the two signals are identical is evidenced in the data sections shown in Supplementary Figure 3.8g. All the fine features are reproduced. We can therefore be certain, that the ML/BL boundary scatters electrons with reversed current path identically.

To check the validity of this procedure, we treat the thermovoltage in a more detailed mathematical description in the following.

Let $V_{\mathrm{STP}}^{0}(x)=V_{\mathrm{Th}}(x)$ be the measured STP signal with no lateral voltage applied, i.e. the pure thermovoltage. For a finite current applied, we then measure for forward and reverse current direction:

$$
V_{\mathrm{STP}}^{\leftarrow}(x)=\mu_{\mathrm{ECP}}^{\leftarrow}(x) / e+\epsilon \cdot V_{\mathrm{Th}}(x) \text { and } V_{\mathrm{STP}} \overrightarrow{\mathrm{C}}(x)=\mu_{\mathrm{ECP}}(x) / e+\epsilon \cdot V_{\mathrm{Th}}(x)
$$

We introduced a constant scaling factor $\epsilon=\epsilon(|I|)$ that scales the thermovoltage due to possible resistive heating of the sample. Note that even for symmetric transport properties $\left[\mu_{\mathrm{ECP}}^{\leftarrow}(x)=-\mu_{\mathrm{ECP}} \vec{\leftarrow}(x)\right] V_{\mathrm{STP}}(x)$ does not show the same behavior with respect to current reversal. It is obvious that thermovoltage and ECP can be retrieved by adding or subtracting the measured maps $V_{\text {STP }}(x)$ and $V_{\text {STP }}^{\leftarrow}(x)$ from each other, i.e. we can now obtain the pure transport field by

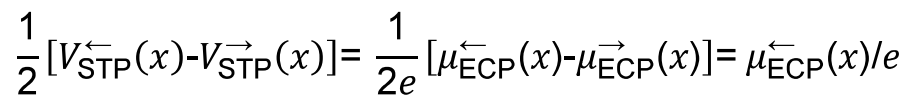

And we can restore the thermovoltage by

$$
V_{\mathrm{Th}}^{\text {restored }}(x)=\frac{1}{2}\left[V_{\mathrm{STP}}^{\leftarrow}(x)+V_{\mathrm{STP}} \underset{\mathrm{P}}{\leftarrow}(x)\right]=\epsilon \cdot V_{\mathrm{Th}}(x)
$$

This evaluation method works of course only if the transport field for both directions is purely symmetric. To test the transport field at the $\mathrm{ML} / \mathrm{BL}$ boundary for a possible asymmetric voltage drop (i.e., the voltage drop caused by electrons traversing from the monolayer to the bilayer is different than for the opposite direction), we assume an asymmetry of the form:

$$
\mu_{\mathrm{ECP}}^{\leftarrow}(\mathrm{x})=-\mu_{\mathrm{ECP}}(\mathrm{x})+\delta \Theta(\mathrm{x})
$$

Where $\Theta(x)$ is a step function at the position of the junction. Then, we would obtain for the transport field and thermovoltage

$$
V_{\mathrm{STP}}^{\text {restored }}(x)=\frac{1}{2}\left[V_{\mathrm{STP}}^{\leftarrow}(x)-V_{\mathrm{STP}} \rightarrow(x)\right]=\mu_{\mathrm{ECP}}^{\leftarrow}(x) / e-\delta \Theta(\mathrm{x}) / \mathrm{e}
$$




$$
V_{\mathrm{Th}}^{\text {restored }}(x)=\frac{1}{2}\left[V_{\mathrm{STP}}^{\overleftarrow{ }}(x)+V_{\mathrm{STP}} \rightarrow(x)\right]=\epsilon \cdot\left[V_{\mathrm{Th}}(x)+\delta \Theta(\mathrm{x}) / \mathrm{e}\right]
$$

So while $V_{\mathrm{ECP}}^{\text {restored }}(x)$ will only show an enlarged voltage drop at the ML/BL junction due to $\delta \Theta(x)$, only if the restored thermovoltage $V_{\mathrm{Th}}^{\text {restored }}(x)$ is compared to $V_{\mathrm{Th}}^{0}(x)$ it can be revealed whether an asymmetric behavior of current transport across the $\mathrm{ML} / \mathrm{BL}$ junction is present. This is not the case in our experiments as shown in Supplementary Figure 3.8g: measured and restored thermovoltage are identical. This is not only true for the difference between $M L$ and $B L$ signals but even most of the fine features are reproduced. The residual deviations are caused by the inherent difficulty to exactly align two STM traces measured at different times due to piezo nonlinearities.

We can therefore be certain that our measurements do not show any asymmetry of transport properties of the ML/BL junction with respect to the direction of current transport across. This is in agreement with most other reports [57, 80]. Recent studies by Clark et al. that report an asymmetry caused by a Friedel oscillationinduced energy gap claim this exclusively in a high voltage/current density range significantly higher than ours[29].

Both ML/BL-steps in Supplementary Figure 3.8f (the left and the right end of the bilayer graphene island) yield similar voltage drops of $147(5) \mu \mathrm{V} / 161(5) \mu \mathrm{V}$ resulting in a step resistance of 12(1) $\Omega \mu m / 13(1) \Omega \mu \mathrm{m}$ (using the local slopes and a macroscopic current density of $j_{\mathrm{mac}}= \pm 12 \mathrm{Am}^{-1}$ ).

\subsubsection{Supplementary Note 2: Exclusion of tip jumping artefacts.}

For the interpretation of the data, it is important to exclude the possibility of tip jumping artefacts.[145] These effects stem from blunt tips or multiple tips and can lead to measured voltage drops that deviate from the actual electrochemical potential at the position of the tip. This appears usually in topographically rough areas as e.g. steps, wrinkles and grain boundaries and is demonstrated in Supplementary Figure 3.10. Far away from the step (Supplementary Figure 3.10a), where the topography is flat, the tunneling current is dominated by an area that is directly below the tip. This changes when the tip is getting closer towards the step on the lower terrace (Supplementary Figure 3.10b). Here, contributions from the step affect not only the topography measurement but also a potentiometry measurement. This leads to an incorrect electrochemical potential at this point. Fortunately, the shift of the potential we observe in the manuscript takes place in the bilayer, which is the higher terrace. As can be seen from Supplementary Figure 3.10c, as soon as the tip reaches the upper layer, the contribution of the step and the lower layer can be neglected, since their contribution decays exponentially with distance. As can be seen from Figure 3.5c in the manuscript, we first see the topographic onset of the bilayer and then the gradual change of the potential. Therefore, we can exclude tip jumping artefacts to alter the ECP from the very start of the bilayer. 
A second possible artefact can originate from multiple tips, but this would also be visible in the topographies, especially at the step.

\subsubsection{Supplementary Note 3: Fitting procedure for the lateral posi- tion of the voltage drop.}

To evaluate the lateral shift of the potential with respect to the topographic step, both are fitted to step functions.

$$
\begin{gathered}
V_{\mathrm{STP}}(x)=\Delta V_{\mathrm{STP}} \cdot\left(1+e^{\frac{x-x_{0}}{L_{\Delta V}}}\right)^{-1}+\Delta V_{\text {offset }} \\
h(x)=\Delta z \cdot\left(1+e^{\frac{x-x_{0}}{L_{\Delta z}}}\right)^{-1}+\Delta z_{\text {offset }}
\end{gathered}
$$

Here, $x_{0}$ is the absolute position of the transition which we defined at $50 \%$ of its height (FWHM-point). $V_{\text {STP }}$ and $\Delta z$ are the amplitudes, $L_{\Delta z}$ and $L_{\Delta V}$ describe the spatial extension of the topography and the drop in the ECP, respectively. This fitting was done for all lines of an STP-measurement. For each fitted line the average of 6 data sections was used as input to decrease the influence of fluctuations. All steps have been analyzed in this manner, all with a spatial resolution of $\leq 0.5$ $\mathrm{nm} . L_{\Delta V}, L_{\Delta z}$ and the lateral offset $\Delta x=x_{0, V_{\mathrm{STP}}}-x_{0, \mathrm{~h}}$ averaged for the two types of investigated junctions are given in Supplementary Table 3.1. As already mentioned in the manuscript, the average lateral offset $\langle\Delta x\rangle$ of the potential is significantly different from zero for the ML/BL-transition only. Also the spatial extension $\langle L\rangle_{\Delta V}$ of this transition is wider than for the ML/ML-case leading to the smooth increase in potential as shown in the manuscript (see Figure 3.5c).

\subsubsection{Supplementary Note 4: Simulation of the shifted voltage drop.}

The lateral extended potential shift of the voltage drop is first described by a resistor network. In Supplementary Figure 3.11 the equivalent circuit diagram is shown. We employ 5 different types of resistors: for the monolayer $R_{\mathrm{ML}}$, for the step $R_{\text {Step }}$, the upper bilayer sheet $R_{\mathrm{BL} \uparrow}$, the lower bilayer sheet $R_{\mathrm{BL} \downarrow}$ and the interlayer resistance $R_{\perp}$ between the bilayer sheets.

The values for $R_{\mathrm{ML}}$ and $R_{\mathrm{BL}}=1 /\left(\frac{1}{R_{B L \uparrow}}+\frac{1}{R_{B L \downarrow}}\right)$ are obtained by fits to the experimental slope of the sheets. Here, we use sheet resistances of $\rho_{\mathrm{ML}}=360 \Omega$ and $\rho_{\mathrm{BL}}=240 \Omega$ obtained from the data in Figure 3.5 in the manuscript (In that figure the voltage drop within the bilayer is not well resolved due to the small length scale. The value of $240 \Omega$ is deduced from observations on a larger scale, e.g. Figure $3.4 e)$. This is in good agreement with other reports.[30, 80] However, as a result from our own measurements, we should state that these values can vary significantly on a local scale. Applying this model the resistance is simply given by 


$$
R_{\mathrm{ML}}=\rho_{\mathrm{ML}} \cdot \frac{L}{W} \quad R_{\mathrm{BL}}=\rho_{\mathrm{BL}} \cdot \frac{L}{W}
$$

Here, $L$ is the length of a segment between two resistors and $W$ the width of the system. Since all resistances scale with $L / W$, its choice does not influence the shape of the potential. The n-doping of epitaxial graphene on $\mathrm{SiC}$ is induced by the charged buffer layer. As a consequence the charge carrier concentration and hence the conductivity [136] of the upper bilayer sheet are decreased by a factor of 10 as shown by photoemission studies $[137,138]$. Consequently, we assume for the resistances

$$
\begin{aligned}
& R_{\mathrm{BL} \uparrow}=(\alpha+1) \cdot R_{\mathrm{BL}} \cdot \frac{L}{W} \\
& R_{\mathrm{BL} \downarrow}=\frac{(\alpha+1)}{\alpha} \cdot R_{\mathrm{BL}} \cdot \frac{L}{W} \\
& R_{\mathrm{BL} \uparrow}{ }^{-1}+R_{\mathrm{BL} \downarrow}{ }^{-1}={R_{\mathrm{BL}}}^{-1}
\end{aligned}
$$

With the doping factor $\alpha=\rho_{\mathrm{BL} \uparrow} / \rho_{\mathrm{BL} \downarrow}$. Here we use for now a decrease by $\alpha=10$ as stated above. To describe the voltage drop directly at the step we use the single resistance $R_{\text {Step }}$ which is caused by the detachment of the layer. It can be calculated from the line resistance of the step $\rho_{\text {Step }}$ by

$$
R_{\text {Step }}=\frac{\rho_{\text {Step }}}{W}
$$

Here, we used $\rho_{\text {Step }}=3 \Omega \mu \mathrm{m}$ which is comparable to the resistance of a ML/MLstep of that height in agreement with $\mathrm{Ji}$ et al.[80] and our own results.

The last parameter in the simulation is the interlayer resistance $R_{\perp}$ which we describe in terms of the ratio $\kappa$ with respect to the bilayer sheet resistance:

$$
\rho_{\perp}=\kappa \cdot \rho_{\mathrm{BL}, \mathrm{Bulk}}
$$

By introducing the bulk resistivity of the bilayer sheet $\rho_{\mathrm{BL}, \mathrm{Bulk}}=\rho_{\mathrm{BL}} \cdot d$, with the thickness of a graphene/graphite layer $d=0.34 \mathrm{~nm}$, we can write the interlayer resistance in terms of

$$
R_{\perp}=\rho_{\perp} \cdot \frac{d}{W \cdot L}=\kappa \cdot \rho_{\mathrm{BL}} \cdot \frac{d^{2}}{W \cdot L}
$$

From literature the ratio between c-axis and in-plane transport for graphite is found to be between $10^{2}-10^{6} \cdot[121]$ A theoretical treatment by Wallace [190] yields a factor of $10^{2}-10^{4}$ depending on temperature. For epitaxial graphene on ruthenium Sutter 
et al. found an increase of $10^{3}$ of the resistance for a contact distance of $10 \mu \mathrm{m}$ depending on whether a ML/BL-step (and consequently interlayer transport) is present or not[170].

We performed simulations for different values of the ratio $\kappa$. In Figure 3.12a we show the results of this simulation. The best fit with the experimental data was obtained by $\approx 1 \cdot 10^{3}$ which fits very well to the literature values. In Figure $3.12 \mathrm{~b}$ we also show the dependence of the potential drop on the ratio between $R_{\mathrm{BL} \uparrow}$ and $R_{\mathrm{BL} \downarrow}$ to demonstrate that the inequivalence induced by different doping has an essential impact on the extended potential shift.

All shown potential drops at the step are calculated for the potential of the upper sheet. The potential at positions in the vicinity of the step differs from the lower sheet due to the high interlayer resistance (This is also indicated in Figure 3.7d). However, this is still in agreement with the experiment, since the potential of the surface, i.e. the upper sheet, is the one mainly mapped by the STP.

In addition, Wang and Beasley suggested an analytical model [193] for scanning probe experiments at the contact region between two materials. This model can be applied if the upper bilayer is considered as a separate material with sheet resistance $\rho_{\mathrm{BL}, \uparrow}$, that is in contact with the lower bilayer with sheet resistance $\rho_{\mathrm{BL}, \downarrow}$. The interlayer resistance can then be considered as the contact resistance $R_{\mathrm{C}}$. The voltage drop is then given by

$$
\begin{gathered}
V_{\mathrm{ML}}=-j \cdot \rho_{\mathrm{ML}} \cdot x \\
V_{\mathrm{BL} \uparrow}=-j \cdot R_{\mathrm{C}}\left[\frac{L^{3}}{l_{\mathrm{BL} \uparrow}^{4}}\left(1-e^{-x / L}\right)+\frac{L^{2}}{l_{\mathrm{BL} \uparrow}^{2} l_{\mathrm{BL} \downarrow}^{2}} \cdot x\right] \\
V_{\mathrm{BL} \downarrow}=-j \cdot R_{\mathrm{C}}\left[\frac{L^{3}}{l_{\mathrm{BL} \uparrow}^{4}}+\frac{L^{3}}{l_{\mathrm{BL} \uparrow}^{2} l_{\mathrm{BL} \downarrow}^{2}} e^{-x / L}+\frac{L^{2}}{l_{\mathrm{BL} \uparrow}^{2} l_{\mathrm{BL} \downarrow}^{2}} \cdot x\right]
\end{gathered}
$$

With the current density $j$ and the transfer lengths

$$
\begin{gathered}
l_{\mathrm{BL} \uparrow}=\sqrt{\rho_{\mathrm{BL}, \uparrow} / R_{\mathrm{C}}} \\
l_{\mathrm{BL} \downarrow}=\sqrt{\rho_{\mathrm{BL}, \downarrow} / R_{\mathrm{C}}}
\end{gathered}
$$




$$
L=\frac{l_{\mathrm{BL} \uparrow} \cdot l_{\mathrm{BL} \downarrow}}{\sqrt{l_{\mathrm{BL} \uparrow}^{2}+l_{\mathrm{BL} \downarrow}^{2}}}
$$

Supplementary Figure 3.13a shows the results for this analytical model (black dotted line) together with the results of the resistor network model (black solid line) with the same parameters as used above. The slight deviation of the two is caused by the step resistance that cannot be included easily into the analytical model.

This is the main difference between both approaches. While the resistor network is flexible in modelling the voltage drop, the analytical model is very intuitive and easier to handle. In the limit of small step resistance contribution they lead to the same results. We also plotted the experimental results from Figure $3.5 \mathrm{c}$ in the manuscript. Besides, more analytical results have been plotted for different values for the ratio $\kappa$. The fit of the black curve has been obtained with

$$
\alpha=10 ; \quad \kappa=3 \cdot 10^{3} ; \quad j=10 \mathrm{Am}^{-1} ; \quad \rho_{\mathrm{ML}}=360 \Omega ; \quad \rho_{\mathrm{BL}}=240 \Omega ;
$$

leading to

$$
\rho_{\mathrm{BL} \uparrow}=(\alpha+1) \cdot \rho_{\mathrm{BL}}=2640 \Omega ; \quad \rho_{\mathrm{BL} \downarrow}=\frac{(\alpha+1)}{\alpha} \cdot \rho_{\mathrm{BL}}=264 \Omega
$$

And

$$
R_{\mathrm{C}}=\kappa \cdot \rho_{\mathrm{BL}} \cdot d^{2}=8.3 \cdot 10^{-10} \Omega \mathrm{cm}^{2}
$$

Moreover, the transfer lengths are

$$
l_{\mathrm{BL} \uparrow}=5.9 \mathrm{~nm} \quad l_{\mathrm{BL} \downarrow}=17.7 \mathrm{~nm} \quad L=5.6 \mathrm{~nm}
$$

We find, while these parameters already yield an adequate description of the effect of the extended and shifted voltage drop, the fit to the experimental data can even be improved by tuning the doping factor $\alpha$. For $\alpha=18$ and $\kappa=1.5 \cdot 10^{3}$ we obtain the curve shown in Supplementary Figure 3.13b. Taking this result seriously, we can conclude that the change in doping concentration deviates by more than a factor of 10 between two layers, at least at the ML/BL-interface. The best fit we obtain for

$$
\alpha=18 ; \quad \kappa=1.5 \cdot 10^{3} ; \quad j=10 \mathrm{Am}^{-1} ; \quad \rho_{\mathrm{ML}}=360 \Omega ; \quad \rho_{\mathrm{BL}}=240 \Omega
$$

Leading to the following values 
3.2 Spatial extent of a Landauer residual-resistivity dipole in graphene quantified by scanning tunnelling potentiometry

$$
\rho_{\mathrm{BL} \uparrow}=(\alpha+1) \cdot \rho_{\mathrm{BL}}=3800 \Omega ; \quad \rho_{\mathrm{BL} \downarrow}=\frac{(\alpha+1)}{\alpha} \cdot \rho_{\mathrm{BL}}=211 \Omega
$$

And

$$
R_{\mathrm{C}}=\kappa \cdot \rho_{\mathrm{BL}} \cdot d^{2}=4.2 \cdot 10^{-10} \Omega \mathrm{cm}^{2}
$$

Moreover, the transfer lengths are

$$
l_{\mathrm{BL} \uparrow}=3.1 \mathrm{~nm} \quad l_{\mathrm{BL} \downarrow}=12.8 \mathrm{~nm} \quad L=3.0 \mathrm{~nm}
$$

This fit is also shown in Figure $3.5 \mathrm{c}$ in the manuscript. 


\subsection{Local transport measurements in graphene on $\mathrm{SiO}_{2}$ using Kelvin probe force microscopy}

P. Willke, C. Möhle, A. Sinterhauf, T. Kotzott, H. K. Yu, A. Wodtke and M. Wenderoth

Carbon 102, pp. 470-476, June 2016

DOI: 10.1016/j.carbon.2016.02.067

By using Kelvin Probe Force Microscopy with an additional applied electric field we investigate the local voltage drop in graphene on $\mathrm{SiO}_{2}$ under ambient conditions. We are able to quantify the variation of the local sheet resistance and to resolve localized voltage drops at line defects. Our data demonstrates that the resistance of line defects has been overestimated so far. Moreover, we show that wrinkles have the largest resistance, $\rho_{\text {wrinkle }}<80 \Omega \mu m$. Temperature-dependent measurements show that the local monolayer sheet resistance reflects the macroscopic increase in resistance with temperature while the defect resistance for folded wrinkles is best described by a temperature-independent model which we attribute to interlayer tunneling.

\subsubsection{Introduction}

Due to its fascinating electronic structure, graphene is a promising candidate for future device physics including gas sensors, flexible displays and microelectronics $[3,56]$. Graphene growth by chemical vapor deposition (CVD) and subsequent transfer to $\mathrm{SiO}_{2}$ has become a favored method for large-scale synthesis [101, 103, 209]. During the growth and transfer process structural defects such as wrinkles, grain boundaries and multilayer areas are formed. They can limit the performance of potential devices since they contribute to the resistance and to the heat generation of the sample $[30,44,61,76,181,210,220]$.

In contrast to conventional transport measurements, scanning probe techniques can reveal local voltage drops reflecting enhanced resistance associated with features of a sample's microscopic structure; this is a significant advantage. The transport physics of atomic scale defects in graphene on $\operatorname{SiC}[30,80,193,201]$ and $\mathrm{SiO}_{2}[30$ ] has been elucidated by scanning tunneling potentiometry (STP) for low temperatures (6-77 K) and under ultra-high vacuum (UHV) conditions and been analyzed theoretically[16, 108]. In contrast, at room temperature and under environmental conditions increased phonon-scattering dominates the graphene sheet resistance [25] while phase coherent transport processes vanish [200, 210].

Using an atomic force microscope (AFM) the method of Kelvin probe force microscopy (KPFM) has been used to map the electrostatic potential in graphene[43, 140, 
211], functionalized and chemically derived graphene[98, 208] and other systems[139, 214]. Thus, AFM techniques working under ambient conditions and room temperature are a versatile tool for analyzing the microscopic transport properties in graphene under conditions relevant for device physics.

For graphene, the KPFM technique has been limited so far to the contactless analysis of the local sheet resistance and to the voltage drop at graphene contacts. In this work we quantify for the first time the resistance of a single line defect in graphene under ambient conditions by KPFM. Being smaller than $80 \Omega \mu \mathrm{m}$, we find that most studies overestimate their influence on the total sample resistance. Moreover, we can map local resistances as a function of temperature by using joule heating in our sample.

\subsubsection{Results and discussion}

\subsubsection{Evaluation of KPFM transport measurements}

The experimental setup is sketched in Figure 3.14a. A graphene sample on $\mathrm{SiO}_{2}$ is investigated by AFM. Additionally, a potential $V_{\text {Bias }}$ is applied via two gold contacts inducing a current across the sample. The IV-characteristic of the device is depicted in Figure 3.14b showing an increasing non-linearity outside the interval $\pm 6 V$, since the sample resistance increases due to increased temperature induced by Joule-heating. The extracted macroscopic resistance $R(T)=V / I(V, T)$ is depicted in Figure 3.14c, now explicitly as a function of temperature (see methods).

(a)

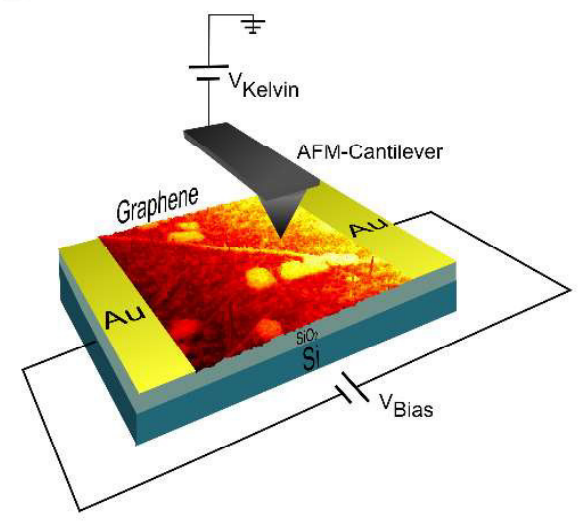

(b)

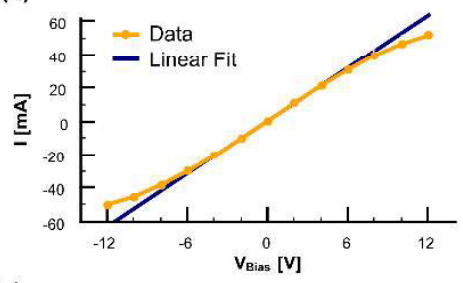

(c)

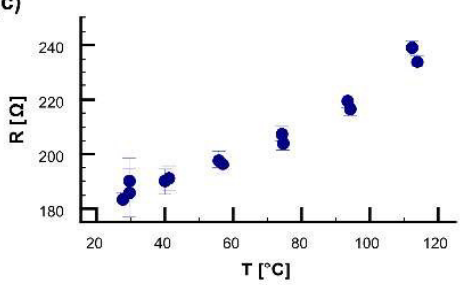

Figure 3.14: KPFM-setup for local transport measurements. (a) Sketch of the experimental setup. Graphene sample is contacted in two-point geometry allowing to apply $\mathrm{V}_{\text {Bias }}$ across the sample while $V_{\text {Kelvin }}(x, y)$ is measured by the AFM. (b) IV-characteristic of the device. The non-linearity for higher $V_{B i a s}$ is induced by the increase in temperature $T$ due to Joule-heating. (c) Absolute device resistance as a function of temperature $T$ derived from the data points in (b). 
To analyze the behavior of the voltage drop on a microscopic scale (also as a function of temperature) we make use of KPFM. Here, a voltage $V_{\text {Kelvin }}$ is applied on the tip side to compensate the electrostatic interaction between tip and sample. In most KPFM experiments no external bias voltage $V_{\text {Bias }}$ is applied and the measured quantity $V_{\text {Kelvin }}$ is equal to the contact potential difference $V_{C P D}$ leading to

$$
\mathrm{V}_{\text {Kelvin }}(\mathrm{x}, \mathrm{y})=\mathrm{V}_{\mathrm{CPD}}(\mathrm{x}, \mathrm{y})=\frac{1}{\mathrm{e}}\left[\Phi_{\text {tip }}-\Phi_{\text {sample }}(\mathrm{x}, \mathrm{y})\right]
$$

where $\Phi_{\text {tip }}$ and $\Phi_{\text {sample }}$ are the work functions of the tip and sample, respectively. The contact potential difference $\mathrm{V}_{\mathrm{CPD}}(\mathrm{x}, \mathrm{y})$ of a $30 \mu \mathrm{m} \times 30 \mu \mathrm{m}$ area is shown in Figure 3.15b (topography shown in Figure 3.15a). Here, the most striking feature is the work function difference between the mostly monolayer graphene covered surface compared to many small islands of bilayer graphene. While $\mathrm{V}_{\mathrm{CPD}}(\mathrm{x}, \mathrm{y})$ does not yield information about the electron transport, it serves as the reference for no applied current and allows additional insight into the electronic structure of the sample. By applying now the additional external bias voltage $V_{\text {Bias }}$ the drop of the electrostatic potential is superimposed on $\mathrm{V}_{\mathrm{CPD}}$ :

$$
\mathrm{V}_{\text {Kelvin }}(\mathrm{x}, \mathrm{y})=\mathrm{V}_{\mathrm{CPD}}(\mathrm{x}, \mathrm{y})+\mathrm{V}_{\text {Bias }}(\mathrm{x}, \mathrm{y})
$$

This is shown in Figure 3.15c and $d$ with an applied external bias of $V_{\text {Bias, }}=+4 \mathrm{~V}$ and $\mathrm{V}_{\text {Bias, }}=-4 \mathrm{~V}$, respectively. Both images already demonstrate an apparent problem for the extraction of transport information: since the variation of $\mathrm{V}_{\mathrm{CPD}}(\mathrm{x}, \mathrm{y})$ is still present in the images, a direct evaluation is difficult. However, under the constraint of symmetric transport $\left(\mathrm{V}_{\mathrm{Bias},+}=-\mathrm{V}_{\mathrm{Bias},-}\right)$ the pure transport signal can be obtained by subtraction of opposite applied bias voltages:

$$
\mathrm{V}_{\text {Transport }}(\mathrm{x}, \mathrm{y})=\frac{1}{2}\left[\mathrm{~V}_{\text {Kelvin }}^{+}(\mathrm{x}, \mathrm{y})-\mathrm{V}_{\text {Kelvin }}^{-}(\mathrm{x}, \mathrm{y})\right]
$$

with $\mathrm{V}_{\text {Kelvin }}^{ \pm}(\mathrm{x}, \mathrm{y})=\mathrm{V}_{\text {Kelvin }}\left(\mathrm{x}, \mathrm{y}, \mathrm{V}_{\text {Bias }, \pm}\right)$.

This effectively cancels the influence of $\mathrm{V}_{\mathrm{CPD}}(\mathrm{x}, \mathrm{y})$ and is shown in Figure 3.15e. Now, the voltage drop is dominated by a linear gradient across the whole image. This evaluation method is equivalent to the elimination of thermovoltage in scanning tunneling potentiometry that we introduced elsewhere [201] and is similar to our recently reported work where KPFM was applied to evaluate the graphene/gold-contact resistance [43]. Yan et al. also subtracted $V_{\text {Kelvin }}^{+}(x, y)-$ $\mathrm{V}_{\mathrm{Kelvin}}^{\mathrm{VV}}(\mathrm{x}, \mathrm{y})[208]$ which is also valid if the surface potential does not change under the influence of current or temperature. We emphasize that Figure $3.15 \mathrm{e}$ clearly demonstrates that complete $2 \mathrm{D}$ transport maps can be obtained with this method. 
In addition, the contact potential difference $\mathrm{V}_{\mathrm{CPD}}(\mathrm{x}, \mathrm{y})$ can be recovered from the maps in Figure 3.15c and $d$ by simple addition.

$$
V_{\mathrm{CPD}}^{\text {restored }}(\mathrm{x}, \mathrm{y})=\frac{1}{2}\left[\mathrm{~V}_{\mathrm{Kelvin}}^{+}(\mathrm{x}, \mathrm{y})+\mathrm{V}_{\text {Kelvin }}^{-}(\mathrm{x}, \mathrm{y})\right]
$$

Comparing Figure $3.15 \mathrm{f}$ with the original contact potential difference in Figure $3.15 \mathrm{~b}$, we see that the assumption of symmetric transport is valid [201]. Figure $3.15 \mathrm{~g}$ shows the averaged potential drop across the sample in the $\mathrm{x}$ - and $\mathrm{y}$-directions. Clearly, the voltage drop occurs mainly in $x$-direction and much less $(7 \%)$ along the y-direction.
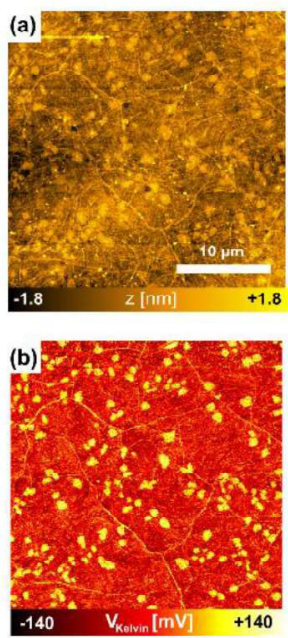
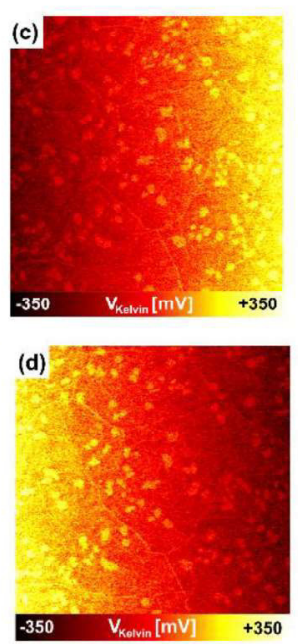
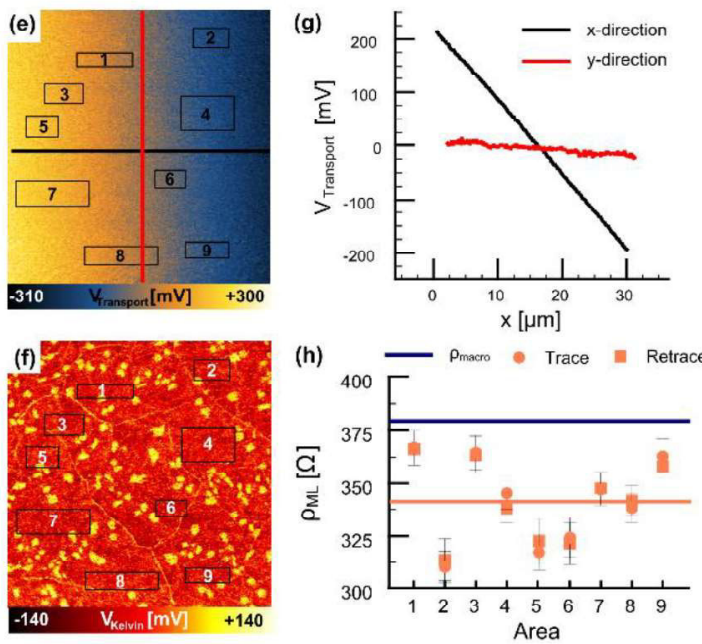

Figure 3.15: AFM transport study on a scale of $30 \mu \mathrm{m} \times \mathbf{3 0} \mu \mathrm{m}$. (a) Topography image of graphene on $\mathrm{SiO}_{2}$. (b) $\mathrm{V}_{\text {Kelvin }}$ without applied bias voltage $\mathrm{V}_{\text {Bias }}$. (c) $V_{\text {Kelvin }}$ for additional applied bias voltage $V_{\text {Bias, }+}=+4 \mathrm{~V}$ and (d) $V_{\text {Bias,- }}=-4 \mathrm{~V}$. (e) $V_{\text {Transport }}$ evaluated by subtracting the KPFM data in (c) and (d). (f) Addition of (c) and (d) reveals the reconstructed $V_{\text {Kelvin }}$-measurement equivalent to the case of no applied bias voltage in (b). (g) Voltage drop in x-direction (black) and y-direction (red) by averaging over the whole image in (e) along the indicated lines. (h) Local monolayer sheet resistance $\rho_{\mathrm{ML}}$ for 9 different areas indicated in (e) and (f). Darkblue line indicates the macroscopic sheet resistance $\rho_{\text {macro }}$ while the yellow line is the average of the local measurements $\rho_{\mathrm{ML}}$.

Variations in the voltage drop are found on a local scale. We calculated the local sheet resistance $\rho_{\mathrm{ML}}=\mathrm{E}_{\mathrm{ML}} / \mathrm{j}_{\text {macro }}$ with the electric field on the monolayer areas $\mathrm{E}_{\mathrm{ML}}$ and the macroscopically measured current density $\mathrm{j}_{\text {macro }}$ for several areas indicated in Figure 3.15e and $\mathrm{f}$. The electric field on the monolayer areas can be calculated by $\mathrm{E}_{\mathrm{ML}}=\Delta \mathrm{V} / \Delta \mathrm{x}$, the slope of the voltage drop $\Delta \mathrm{V}$ over the distance $\Delta \mathrm{x}$. Besides, the macroscopic current density is defined as $\mathrm{j}_{\text {macro }}=\mathrm{V}_{\text {Bias }} \cdot \mathrm{W} / \mathrm{R}$ with the width $W$ of the sample and the sample resistance $R$ as shown in Figure 3.15c. The result can be found together with the macroscopic sheet resistance $\rho_{\text {macro }}$ in Figure 3.15h. Two observations can be made. First, the local monolayer resistance is 
$\approx 10 \%$ smaller than the macroscopically measured sheet resistance $\rho_{\text {macro }}$. Reasons for this lower value are the observed voltage drop in y-direction which can be introduced by small deviations in the alignment of the sample. Possibly, the contact resistance between gold and graphene can contribute to the voltage drop which is however relatively small [43]. In addition, the influence of localized defects as ML/BL-interfaces, steps and wrinkles can give a significant contribution to the total resistance as in the case of graphene on $\mathrm{SiC}[80,201]$. The comparably small difference to $\rho_{\text {macro }}$ already limits this influence to $\leq 10 \%$. The second observation is that the monolayer sheet resistance $\rho_{\mathrm{ML}}$ varies by up to $8 \%$ from the average value $\left\langle\rho_{\mathrm{ML}}\right\rangle=342 \Omega$. Reasons for this can be a different density of defects such as smaller wrinkles present on the sample as well as local differences in doping and differences in coupling to the substrate. Moreover, it has been shown that the size of single graphene grains has an influence on the total resistance [44]. Since grain boundaries are not visible within AFM measurements we cannot evaluate this quantitatively, while the grain size $(<10 \mu \mathrm{m})$ is clearly smaller than the image size. The evaluation of any local resistance from the voltage drop is only legitimate, if the local current density is sufficiently homogeneous and not locally varying due to e.g. defects. In order to estimate the effects of inhomogeneities in the current density, we have performed resistor network simulations of the system (see Supporting Information). The current is found to be mainly homogeneous (coefficient of variation $3.8 \%$ ), thus employing the macroscopic quantity $\mathrm{j}_{\text {macro }}$ is justified and only holds a small error for the values of local resistances.

\subsubsection{Temperature dependence of local resistances}

As already discussed for the macroscopic case, the macroscopic resistance $\rho_{\text {macro }}$ increases with temperature $T$ due to Joule-heating. In Figure 3.16 we demonstrate that this is also true at the microscopic scale. For the two monolayer areas indicated in the topography (Figure 3.16a) and the KPFM map (Figure 3.16b) we measured the electric field $E_{M L}$ for different bias voltages $V_{\text {Bias }}$ in the range between $\pm 4 \mathrm{~V}$ and $\pm 12 \mathrm{~V}$. The latter can be expressed as the macroscopic current density $\rho_{\text {macro }}$. Figure $3.16 \mathrm{c}$ depicts the electric field $E_{\mathrm{ML}}$ as a function of $j_{\text {macro }}$ which would be linear if temperature did not vary and for the Ohmic case. The deviation from linearity reveals the temperature dependence of the resistance. In Figure 3.16d we show the calculated temperature dependent sheet resistance for both areas together with that of the macroscopic sample. All show a similar slope. The increase in sheet resistance with temperature for graphene on $\mathrm{SiO}_{2}$ can be explained by additional contributions from acoustic phonons $(\rho(T) \propto T)$ and from the activation process of two surface phonon modes in $\mathrm{SiO}_{2} \rho(\mathrm{T}) \propto$ $\left(\mathrm{e}^{\frac{E_{0}}{\mathrm{k}_{\mathrm{B}} \mathrm{T}}}-1\right)^{-1}[25]$. The fits to the data using this model are shown as lines in Figure $3.16 \mathrm{~d}$. The macroscopic change and the two microscopic values yield similar values for the activation energy $E_{0}$. The variation of the curves in Figure $3.16 \mathrm{~d}$ only 
stems from the temperature-independent part $\rho_{0}$ varying in the range as discussed in Figure 3.16h (see Supporting Information). This part is amongst others depending on gate voltage/electron concentration [25]. While no gate voltage has been applied in this experiment, local changes in doping as well as different concentration of atomic scale defects can lead to local variations of this term.


Figure 3.16. Temperature-dependence of local sheet resistance $\rho_{\mathrm{ML}}(\mathrm{T})$. (a) Topography map and (b) KPFM map without applied bias voltage. (c) Electric field $E_{\mathrm{ML}}$ as a function of $j_{\text {macro }}$ for the two areas indicated in (b). Lines are fitted to the data points of lowest current density. (d) Local monolayer sheet resistances $\rho_{\mathrm{ML}}(\mathrm{T})$ as a function of temperature T calculated from the data in (c). Black points show the macroscopic sheet resistance $\rho_{\text {macro }}(\mathrm{T})$ derived from the data in Figure $3.14 \mathrm{c}$ and the device geometry. Lines indicate the fit to the model described in the text.

\subsubsection{Resistance of line defects}

A variety of line defects, such as grain boundaries, ML/BL-interfaces and wrinkles can be found on our samples; however, they hardly influence the transport compared to e.g. graphene on $\mathrm{SiC}[80,201]$. As demonstrated in Figure 3.15e, the main voltage drop is found on the free graphene terraces. Nevertheless, by carefully analyzing the voltage drop in the vicinity of a defect, their small contributions to the resistance can be observed.

One of the most prominent defects are folded graphene wrinkles, one of which can be seen in the middle of Figure 3.16a-b (Defect \#4). According to Zhu et al. these are formed when a wrinkle's height becomes large enough to result in instability and collapse [220]. It then forms a local quasi-trilayer (TRL) graphene region. 
Therefore, they are comparably wide yet smaller in height compared to unfolded wrinkles (Figure 3.17a left/middle). The folded graphene wrinkles analyzed here are between 150 and $300 \mathrm{~nm}$ wide and 0.9-1.2 nm in height similar to those reported in [220]. Their anticipated structure can be seen in Figure 3.17a according

(a)

(b)
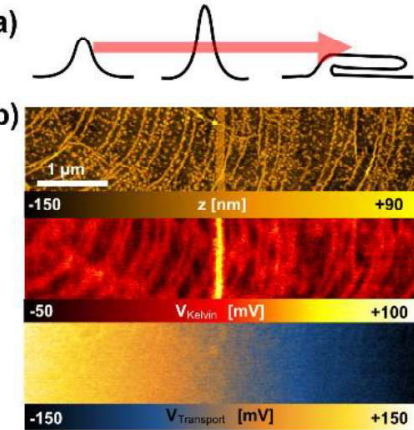

(c)

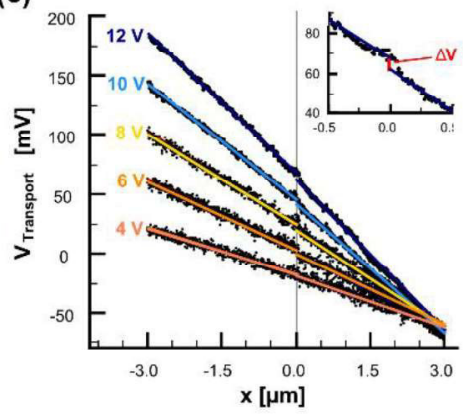

(d)

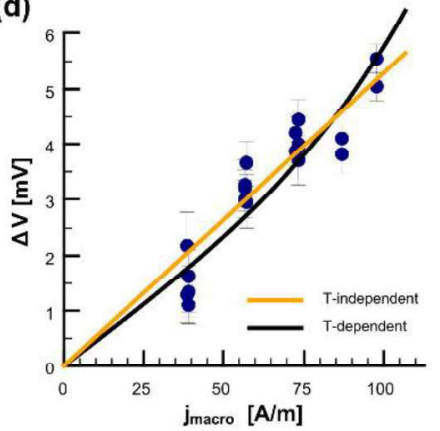

Figure 3.17: Voltage drop at a folded graphene wrinkle. (a) Sketch of the formation of a folded graphene wrinkle. In the folded region three layers of graphene are effectively on top of each other. (b) Topography, KPFM and transport map $\left(V_{\text {Bias }}=12 \mathrm{~V}\right)$ for the folded graphene wrinkle in Figure 3.16 (\#4). (c) Averaged voltage drop $V_{\text {Transport }}$ across the data shown in (b) taken for different applied bias voltages $V_{\text {Bias. }}$ The $x$-axis has been adjusted so that the wrinkle is located at $x=0 \mu \mathrm{m}$ (gray vertical line). Colored lines indicate linear fits to the left and right side of the wrinkle. The inset displays the voltage drop in the vicinity of the wrinkle for $V_{\text {Bias }}=12 \mathrm{~V}$ with the voltage drop $\Delta V$ located at the position of the wrinkle. (d) Voltage drop $\Delta \mathrm{V}$ for the data in (c) as a function of macroscopic current density $\mathrm{j}_{\text {macro }}$. The orange (black) line shows the temperature-independent (temperature-dependent) fit to the data.

to the model of [220]. In Figure 3.17b, we show the topography of the folded wrinkle from the data set in Figure 3.16 along with the KPFM and the transport map. The KPFM map clearly shows a strong contrast on the wrinkle indicating its different electronic structure. The work function difference associated with the folded wrinkles $\left[\Delta \Phi_{\text {Wrinkle-ML }}=(85 \pm 5) \mathrm{mV}\right]$ in Figure $3.16 \mathrm{~b}$ is demonstrably higher than that for the bilayer $\left[\Delta \Phi_{\mathrm{BL}-\mathrm{ML}}=(71 \pm 5) \mathrm{mV}\right]$. While $\Delta \Phi_{\mathrm{BL}-\mathrm{ML}}$ is in excellent agreement with other reported values [221], the higher value for $\Delta \Phi_{\text {Wrinkle-ML }}$ is a strong evidence for the TRL-like nature of the folded wrinkle [221]. In Figure 3.17c we present averaged sections through the transport map for different applied bias voltages. For a defect resistance $\rho_{\text {Wrinkle }}$ we expect a step-like jump in the potential $\Delta V$ at the position of the wrinkle (gray line). Note that a defect resistance for a 1D defect is given in $\Omega \mu \mathrm{m}$ in contrast to the sheet resistance $\rho_{\mathrm{ML}}$ due to its difference in dimension. The jump in potential $\Delta \mathrm{V}$ can be clearly seen in the inset for $\mathrm{V}_{\text {Bias }}=$ $12 \mathrm{~V}$. For a local scatterer at constant temperature, the size of the potential jump effectively scales with $\mathrm{V}_{\mathrm{Bias}}[80]$, since

$$
\Delta V=\rho_{\text {Wrinkle }} \cdot j_{\text {macro }}\left(V_{\text {Bias }}\right)
$$


3.3 Local transport measurements in graphene on $\mathrm{SiO} 2$ using Kelvin probe force microscopy

To evaluate $\Delta \mathrm{V}$ we fitted straight lines to the voltage drop over the $\mathrm{ML}$ sheets to the left and to the right of the wrinkle and determined their difference at the position of the wrinkle as shown in the inset of Figure 3.17c. While the evolution of the voltage drop within the defect could give access to further information on the scattering mechanism [201], we cannot exclude crosstalk from $V_{\text {Kelvin }}$ (Figure 3.17b) that changes significantly on the wrinkle and therefore we do not discuss this further here. In Figure $3.17 \mathrm{~d}$, we plot $\Delta \mathrm{V}$ as a function of macroscopic current density $\mathrm{j}_{\text {macro }}$. We compare this to predictions of two models of transport: one reflecting the temperature-dependent sheet resistance of the monolayer graphene $\rho_{\mathrm{ML}}$ (black line) and one reflecting a temperature-independent voltage drop (orange line). We find that the observations are most consistent with a T-independent transport model (see Supporting Information). This is supported by the results of Grosse et al. who found a large increase in Joule-heating at localized scatterers [61]. Thus, it is possible that the temperature of the folded wrinkles is higher than for the sheet itself. Hence, if $\rho_{\text {Wrinkle }}$ were temperature dependent, deviations from the linear behavior of Figure 3.17d would be larger than expected. Consequently, we conclude that for the localized scatterer the underlying scattering mechanism shows either a significantly smaller temperature-dependence or is completely independent of temperature.

From the best fit to the data we determine the wrinkle defect resistance, $\rho_{\text {Wrinkle }}=$ $(51.9 \pm 2.4) \Omega \mu \mathrm{m}$. The lowest reported experimental resistance value for a graphene wrinkle is $200 \Omega \mu \mathrm{m}$ for a $20 \mathrm{~nm}$ wide wrinkle [30]. Theoretically, the folded graphene wrinkle was postulated to have a defect resistance 200-300 $\Omega \mu \mathrm{m}$ dominated by interlayer tunneling using nonequilibrium Green's function method [220]. Our measurements suggest that the influence of this type of wrinkle has likely been overestimated up to now.

In Figure 3.18 we show all significant local defects in the area of Figure 3.16 as well as those from a similar map. All folded graphene wrinkles show a similar defect resistance; furthermore, standing wrinkles have comparable but slightly smaller defect resistances. We cannot exclude the presence of grain boundaries at these positions while it is likely to coincide, since the grain size in these samples is only of the order of several $\mu \mathrm{m}$. On the other hand, since we did not observe any larger localized voltage drops in our sample, the line resistance of grain boundaries must be comparable to or smaller than $80 \Omega \mu \mathrm{m}$, which is again smaller than previous experimental reports [30,61]. 


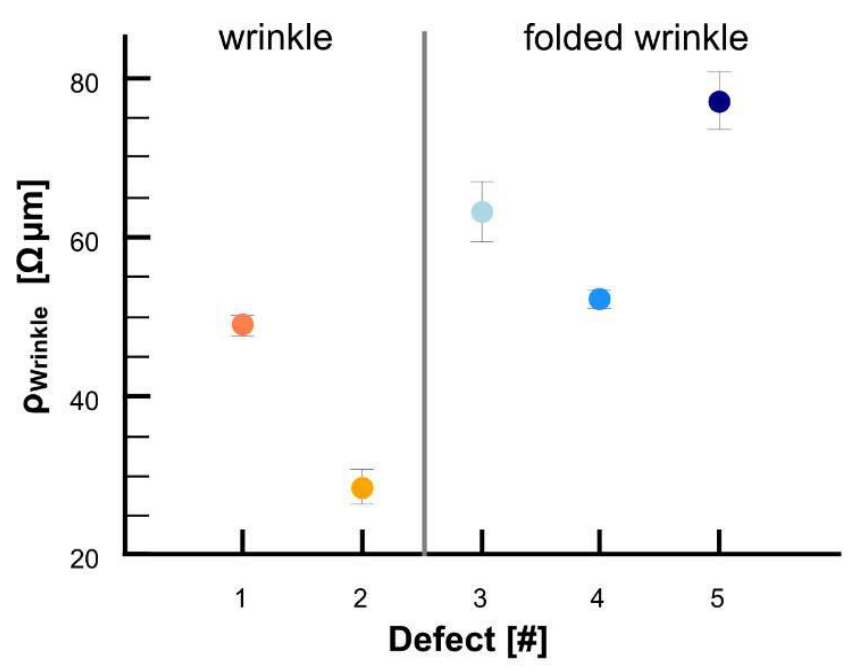

Figure 3.18: Defect line resistance $\rho_{\text {wrinkle }}$ for different wrinkles. For wrinkles \#1 - \#4 the colors indicate their position in Figure 3.16a. Wrinkle \#4 corresponds to that in Figure 3.17. Gray line separates between unfolded (left) and folded graphene wrinkles (right).

We may understand the value of the folded wrinkle resistance if we note that the wrinkle can be modeled by two ML/TRL interfaces. As ML/BL-interfaces are known to have a defect resistance of 10-30 $\Omega \mu \mathrm{m}[80,201]$, it is not surprising that a double ML/TRL interface might exhibit a value about twice as large. A more sophisticated model would have to take into account the particular structure of the defect: in case of no interlayer coupling, the defect resistance results simply from the additional path traveled by the electrons; this can be estimated, $\rho_{\text {Wrinkle }}=2 \cdot \rho_{\mathrm{ML}}$. $\mathrm{L}_{\text {Wrinkle }} \approx 200 \Omega \mu \mathrm{m}$. This is not consistent with our observations. To accurately describe lower values of the defect resistance, interlayer tunneling has been proposed theoretically [220].

A folded wrinkle tunneling transport model is confirmed by our results, not only by the lower than expected value of the defect resistance through the folded wrinkle, but also by its temperature-independence. If the electron transport were simply governed by the elongated path, the defect resistance would increase due to the temperature-dependence of $\rho_{\mathrm{ML}}(\mathrm{T})$. On the other hand, for tunneling between graphene sheets the resistance is lowered at elevated temperature due to thermal broadening of the Fermi distribution [183]. However, since this is a second order process the effect is rather small and not observable in our experiments. By modeling the interlayer tunneling process in a classical resistor network model (see Supporting Information) we can estimate the interlayer resistance to be $\mathrm{R}_{\mathrm{C}} \approx 8 \cdot 10^{-8} \Omega \mathrm{cm}^{2}$ which we treated here as a contact resistance between two layers of graphene. This value is $2-3$ orders of magnitudes larger than the bilayer interlayer resistance for graphene on SiC indicating a weaker coupling and a higher potential barrier [201]. Despite this, it is still smaller than, for example, the contact resistance between graphene and gold [43]. 
3.3 Local transport measurements in graphene on $\mathrm{SiO} 2$ using Kelvin probe force microscopy

For the unfolded wrinkles (\#1 and \#2) the scattering mechanism must be a different because the path is almost not elongated and thus tunneling is not favored here. One possibility is the influence of grain boundaries as described above. An alternative explanation might invoke a scattering mechanism influenced by an induced potential by detachment of the graphene from the substrate as shown for steps for graphene on $\mathrm{SiC}[80,108,201]$. For CVD-grown graphene, a local change in doping has been found at wrinkles and grain boundaries on the atomic scale by scanning tunneling spectroscopy $[94,171]$.

\subsubsection{Conclusion}

In summary, we have utilized KPFM to map the spatial evolution of the electrostatic potential for CVD-grown graphene. By additional data processing we could disentangle the transport and electronic contribution to the mapped surface potential. We are able to quantify the local variations of the monolayer sheet resistance and to prove its local dependence on temperature. In contrast, local voltage drops across collapsed wrinkles gave only small contributions to the total resistance and did not depend on temperature. We suggest that this is due to a different scattering mechanism involving interlayer tunneling. All observed voltage drops reflected a line defect associated resistance $<80 \Omega \mu \mathrm{m}$.

\subsubsection{Methods}

We used commercial graphene samples (Graphene Supermarket) on $\mathrm{SiO}_{2}$ $(285 \mathrm{~nm})$ on $\mathrm{p}$-doped Si-substrate with no additional gate voltage applied. While the KPFM measurements were done on one sample only, several samples where checked for similar morphology via normal AFM measurements. Since the samples showed no signs of contamination, no cleaning step was performed. Gold contacts were added in a shadow mask procedure and contacted by aluminum bond wires in a two-terminal geometry. Thus, we prepare large area graphene samples with a width $W=(520 \pm 20) \mu \mathrm{m}$ and length $\mathrm{L}=(260 \pm 20) \mu \mathrm{m}$.

KPFM is realized in a commercial setup (Agilent 5600LS). Atomic force and Kelvin force measurements are performed in tapping mode; amplitude modulation KPFM was used. For KPFM mode we used platinum deposited silicon cantilevers (OLYMPUS OMCL-AC240TM-B2) with a resonant frequency of $70 \mathrm{kHz}$ and a spring constant $2 \mathrm{~N} / \mathrm{m}$.

The calibration of the sample temperature has been done by use of an infrared camera (Wöhler, EasIR-4). During the temperature measurements the same current densities and voltages as later in the KPFM experiments have been applied to the sample and subsequently, the temperature was mapped in the middle of the graphene sample. This yield a quadratic relation $T=(0.0089 \pm 0.0002) m^{2} K / A^{2}$. $j_{\text {macro }}^{2}+(298.9 \pm 0.7) K$ as expected for Joule-heating. While the temperature might not be homogeneous about the sample, e.g. at the contacts, the KPFM maps 
were taken in the middle where also the temperature was measured. Due to the high homogeneity in current density, we assumed Joule heating and thus the temperature to be constant on the ML graphene sheets.

Measurements have been taken over several days and with different tips, especially the data shown in Figure 3.17 demonstrate their reproducibility.

For the evaluation of the local voltage drops, all lines of a map have been shifted along the x-direction to give a straight line as depicted in Figure $3.17 \mathrm{~b}$. Thus, averaging along the $y$-axis improves the statistics and thus the resolution of the voltage drop. This method is valid for a small voltage drop in y-direction which we demonstrated in Figure $3.15 \mathrm{~g}$. For inclined wrinkles a correction factor of $\cos \theta$ has been introduced corresponding to the projection in the direction of the current in order to reproduce the actual voltage drop.

\subsubsection{Acknowledgement}

This work was supported by the Deutsche Forschungsgemeinschaft (DFG) priority program 1459 Graphene.

\subsubsection{Author contributions}

M.W., P.W., A.W. and H.Y. planned the experiments. P.W., A.S., C.M. performed the AFM measurements. T.K. and P.W. performed the resistor network simulations. The manuscript was written through contributions of all authors. All authors have given approval to the final version of the manuscript.

\subsubsection{Supplementary Information}

\subsubsection{Resistor network simulations and current inhomogeneities}

In the data analysis we assume a homogeneous current density $j_{\text {macro }}$ over the sample. To estimate the homogeneity of the current distribution we perform classical resistor network simulations of the transport map as introduced by Homoth et al.[71] The sample system has been modelled corresponding to the topography in Figure $3.15 \mathrm{a}$ by connecting the data points with resistors horizontally and vertically. The area is divided into monolayer, bilayer, and wrinkle areas and to each resistor the value of the experimental results as given in the manuscript is assigned. The potential $V_{\text {Transport }}$ on the left and right image borders is derived by a linear fit of the experimental transport data. The result of the simulated potential map is shown in Figure 3.19a. It is highly dominated by a linear voltage drop. From potential and resistance values the local current in each data point can be calculated. The current map can be found in Figure 3.19b. The edges of defects, i.e. ML/BL steps and wrinkles, are sketched by black lines because the simulation of the currents in these edges depends on the discretization of the model. However, the current flow on free areas is consistently described for the given surface structure including 
defects. The current density is found to be homogeneous over the $30 \mu \mathrm{m} \times 30 \mu \mathrm{m}$ sample as depicted in Figure 3.19c. Both, a Gaussian fit and statistical evaluation of the data, give a coefficient of variation, i.e. a ratio of standard deviation to the mean value of $\sigma / \mu=3.8 \%$ over the whole area for the resistance values from the manuscript $\rho_{\mathrm{ML}}=342 \Omega, \rho_{\mathrm{ML} / \mathrm{BL}}=20 \Omega \mu \mathrm{m}$, and $\rho_{\mathrm{Wr}}=50 \Omega \mu \mathrm{m}$. Hence, the simulation justifies the assumption of a nearly homogeneous current density. An uncertainty of $4 \%$ is included in the calculation of the error of sheet and wrinkle resistances.

Even for an increased wrinkle resistance of $\rho_{\mathrm{Wr}}=200 \Omega \mu \mathrm{m}$, the standard deviation of the current is still given by a value of $6 \%$. However, then the voltage drop over the wrinkle should be in the order of $\Delta V \approx 8 \mathrm{mV}$ for a current density of $\mathrm{j}_{\text {macro }}=$ $39 \frac{\mathrm{A}}{\mathrm{m}}$ which is substantially above the experimental results (see Figure $3.17 \mathrm{~d}$ ).

On the nanoscale the orientation of the current in the free graphene sheet is to very high extent given by the horizontal component, i.e. parallel to the macroscopic current density. Therefore it is legitimate for the evaluation of wrinkle resistances to assume the microscopic current to be running horizontally with the macroscopic current density.

As shown in Figure $3.15 \mathrm{~h}$ in the manuscript, the sheet resistances for different areas vary by up to $8 \%$. We found that even including such variations in the simulations by applying a Gaussian to the resistance values on free areas does not change the uniformity of the current significantly and results in a still moderate coefficient of variation of $6 \%$.

(a)

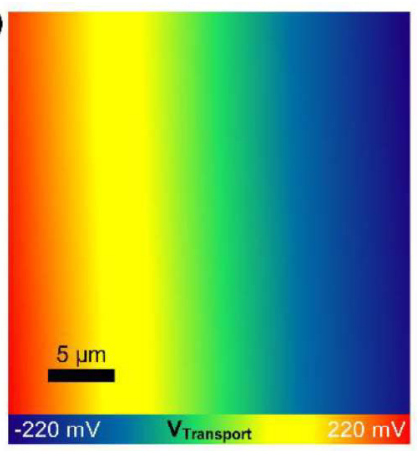

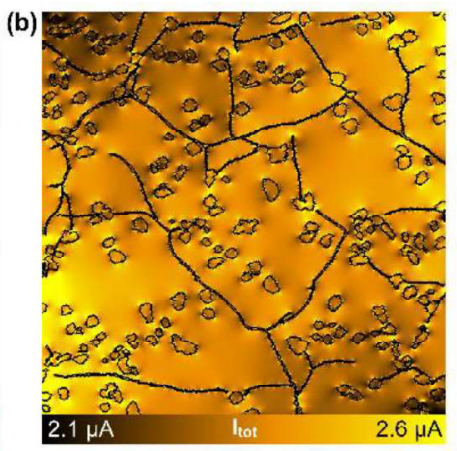

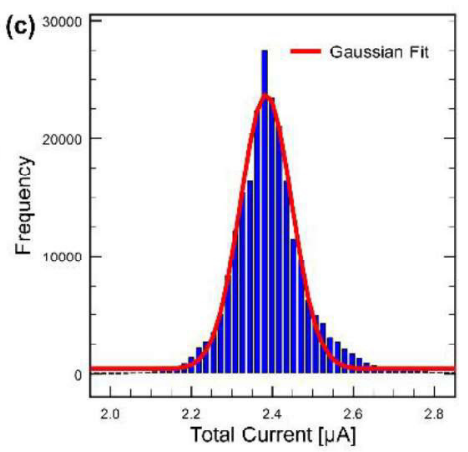

Figure 3.19: Resistor network simulation of the transport map shown as Figure 3.15e with a discretization of $512 \times 512$ data points matching the KPFM resolution. (a) Transport potential $V_{\text {Transport }}$ resulting from a network with resistor values $\rho_{\mathrm{ML}}=342 \Omega, \rho_{\mathrm{ML} / \mathrm{BL}}=$ $20 \Omega \mu \mathrm{m}$, and $\rho_{\mathrm{Wr}}=50 \Omega \mu \mathrm{m}$. (b) Local current map with defects sketched in black. (c) Histogram of total currents of the map shown in (b) with Gaussian fit with mean value $\mu=$ $2.38 \mu \mathrm{A}$ and standard deviation $\sigma=8.95 \cdot 10^{-2} \mu \mathrm{A}$. The relative deviation of $\sigma / \mu=3.8 \%$ shows the homogeneity of the current density over the sample. 


\subsubsection{Modelling the temperature-dependence of graphene on $\mathrm{SiO}_{2}$}

The temperature-dependence of graphene on $\mathrm{SiO}_{2}$ has been discussed by Chen et al.[25] As described in the manuscript, the additional dependence has been introduced by a linear temperature-dependence $\rho_{\mathrm{A}}(\mathrm{T})$ due to acoustic phonons and an exponential contribution due to coupling to phonon-modes in the substrate. In the simplest model this has been expressed as a single contribution.[25] A third contribution $\rho_{0}$ is independent of temperature and thus limits the low-temperature case. Thus, the dependence is given by

$$
\rho(T)=\rho_{0}+\rho_{A}(T)+\rho_{B}(T)
$$

with $\quad \rho_{A}(T)=K_{1} \cdot T=\left(\frac{h}{e^{2}}\right) \frac{\pi^{2} D_{A}^{2} k_{B}}{2 h^{2} \rho_{s} v_{S}^{2} v_{F}} \cdot T \quad$ and $\quad \rho_{B}(T)=K_{2} \cdot\left(\frac{1}{e^{E_{0} / k_{B} T}-1}\right)$

Here, $D_{A}$ is the acoustic deformation potential, $\rho_{S}$ is the $2 D$ mass density of graphene, $v_{S}$ is the velocity for LA phonons and $v_{F}$ is the Fermi-velocity. Using the values in [25] we set $K_{1}=0.0316 \frac{1}{\mathrm{~K}}$. Moreover, $\mathrm{K}_{2}$ has been introduced to take account for the dependence on the gate voltage. Therefore, we also replace it with the value for no gate voltage $K_{2}=5730 \Omega$, as in our experiment. Our best fit parameters for the macroscopic case and the two local dependencies can be found in Table 3.2.

\begin{tabular}{c|cc}
\multicolumn{1}{c}{} & $\boldsymbol{\rho}_{\mathbf{0}}[\boldsymbol{\Omega}]$ & $\mathbf{E}_{\mathbf{0}}[\mathbf{m e V}]$ \\
\hline $\boldsymbol{\rho}_{\text {macro }}$ & 303 & 122 \\
$\boldsymbol{\rho}_{\mathrm{ML}, \mathbf{1}}$ & 260 & 129 \\
$\boldsymbol{\rho}_{\mathrm{ML}, \mathbf{2}}$ & 298 & 127 \\
\hline
\end{tabular}

Table 3.2. Parameters to describe the temperature-dependent resistance.

\subsubsection{Resistor network model for a folded graphene wrinkle}

The concepts of the resistor network model have been introduced elsewhere.[71, 201] Figure 3.20 depicts the equivalent circuit diagram of the resistor network model for the folded graphene wrinkle. $R_{M L}=\frac{W}{L} \cdot \rho_{M L}$ is here the resistance of a segment of monolayer graphene (sheet resistance $\rho_{\mathrm{ML}}=350 \Omega$ ) with length $L$ and width $\mathrm{W}$. We here neglected any possible changes in the charge carrier concentration of the single layers and assumed an equal distribution, so that all three layers have the same resistance. Additionally, interlayer resistance $R_{\perp, 12}=\kappa \cdot R_{M L} \cdot \frac{d^{2}}{W \cdot L}$ can be expressed as a multiple $\kappa$ of the ML resistance $R_{M L}$ (times the dimensionless factor $\frac{d^{2}}{W \cdot L}$ with $d=3.4 \AA$ the graphite layer thickness, that accounts for the different current direction). While $L$ and $W$ are introduced here to define the values 
of the resistance, the results are independent of them as long as the choice of the grid is made small enough.

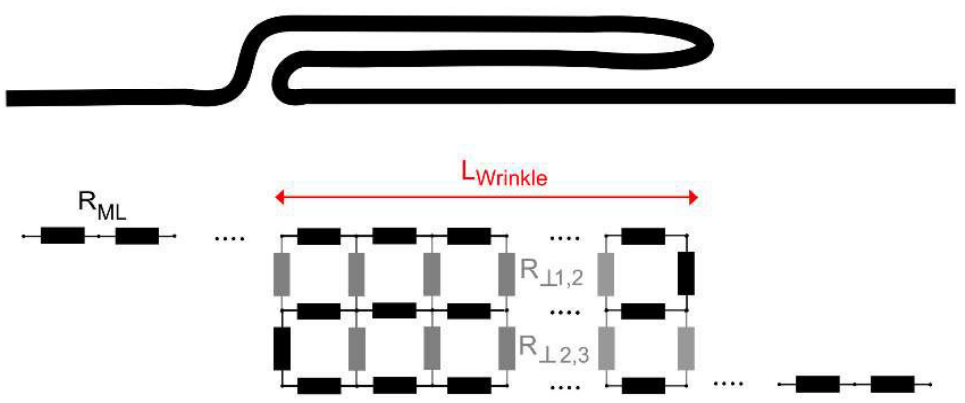

Figure 3.20: Geometry and equivalent circuit diagram of the folded graphene wrinkle. We use $\kappa$ as a fitting factor to the experimental data. $R_{\perp, 12}$ and $R_{\perp, 23}$ are the interlayer resistances for the first and second/second and third layer, respectively.

We now simulate two limiting cases

1. $R_{\perp, 12} \ll R_{\perp, 23}$ : This implicates that the folded wrinkle is only coupled to the upper (or lower) sheet as has been assumed in [220].

2. $R_{\perp, 12}=R_{\perp, 23}$ : This is the case when the wrinkle couples to both sheets and forms a trilayer-like structure as suggested by the KPFM data in the manuscript.

The evaluation of the defect resistance $\rho_{\text {Wrinkle }}$ is performed analogously to the experimental data in the manuscript by fitting linear functions to the regions left and right of the defect and dividing the voltage drop by the current density. Figure 3.21a depicts the result for case 1 and Figure 3.21b for case 2. We show the defect resistance as a function of the wrinkle length $L_{\text {Wrinkle }}$ as well as for different values $\kappa$. For case 1 , two regimes can be distinguished: for small values of $L_{\text {Wrinkle }}$ the increase is linear, since the wrinkle is too short for electrons to tunnel between the layers and thus the transport is still dominated by the in-plane transport. For larger values of $L_{\text {Wrinkle }}$ the resistance saturates, since now the transport is completely dominated by tunneling and thus independent of the length of the wrinkle. This is in agreement with the simulation by Zhu et al.[220] The gray area marks the range of $\mathrm{L}_{\text {Wrinkle }}$ observed experimentally. Consequently, here the transport is already dominated by the tunneling. For case 2 , the transport is even decreasing for long wrinkles, since the transport can take place in all three layers effectively reducing the resistance in the wrinkle (even leading to lower resistances than on the $\mathrm{ML}$ ). The experimentally observed defect resistance is $\rho_{\text {Wrinkle }} \approx 50 \Omega \mu \mathrm{m}$. Thus, we obtain the best fit with $\kappa=1 \cdot 10^{5}$ (case 1 ) and $\kappa=4 \cdot 10^{5}$ (case 2). The resistance between two layers can also be treated as a contact resistance [201] that can be calculated by 


$$
\mathrm{R}_{\mathrm{C}}=\frac{1}{2} \cdot \kappa \cdot \rho_{\mathrm{ML}} \cdot \mathrm{d}^{2}
$$

Leading to

$$
\left.\mathrm{R}_{\mathrm{C}, 1}=2 \cdot 10^{-8} \Omega \mathrm{cm}^{2} \quad \text { (case } 1\right) \quad \mathrm{R}_{\mathrm{C}, 2}=8 \cdot 10^{-8} \Omega \mathrm{cm}^{2} \quad \text { (case 2) }
$$

As stated in the manuscript, this is significantly higher than the contact resistance found for the monolayer bilayer transition in epitaxial graphene $\left(4.2 \cdot 10^{-10} \Omega \mathrm{cm}^{2}\right)$.[201] This can be explained with a weaker coupling than in epitaxial graphene. In fact, since the atomic lattice is not resolved in this experiment, we cannot determine the stacking of the different layers. This should however be crucial for the interlayer tunneling.

(a)

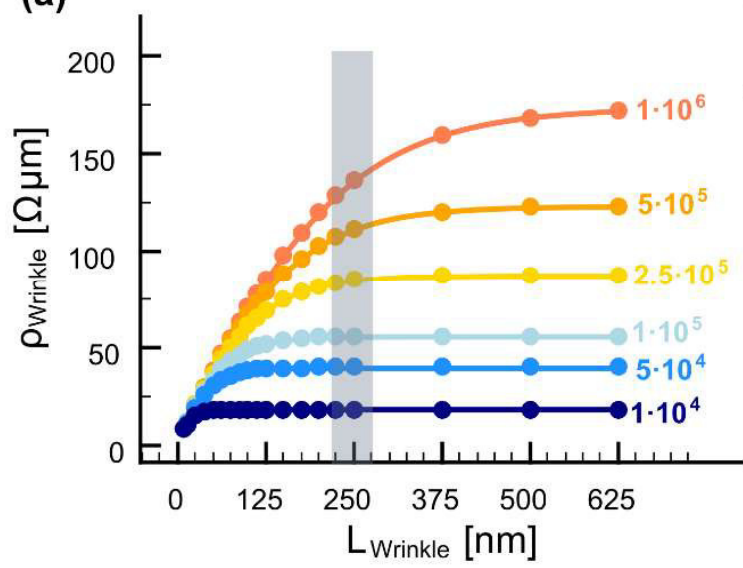

(b)

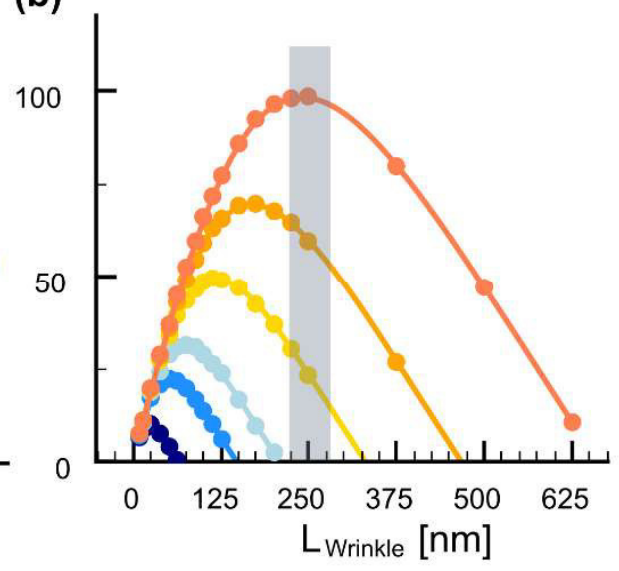

Figure 3.21: Results for the resistor network model of the folded graphene wrinkle with the defect resistance $\rho_{\text {Wrinkle }}$ as a function of wrinkle length $L_{\text {Wrinkle. }}$ (a) Results for a bilayer coupling $\left(R_{\perp, 12} \ll R_{\perp, 23}\right)$ for different values of $\kappa$ between $10^{4}$ and $10^{6}$. (b) Results for trilayer coupling $\left(R_{\perp, 12}=R_{\perp, 23}\right)$ for different values of $\kappa$ between $10^{4}$ and $10^{6}$ [same as in (a)]. Gray area marks the experimentally investigated length of folded graphene wrinkles.

\subsubsection{Temperature-dependence of the step resistance of folded bi- layer wrinkles}

In Figure 3.22 we show the data from Figure 3.22d in the manuscript, now explicitly the step resistance of the folded graphene wrinkle $\rho_{\text {Wrinkle }}$ as a function of temperature $\mathrm{T}$. This is calculated by

$$
\rho_{\text {Wrinkle }}=\frac{\Delta \mathrm{V}}{\mathrm{j}_{\text {macro }}}
$$

and the measured relation between the temperature and the current density is given by $\mathrm{T}=(0.0089 \pm 0.0002) \frac{\mathrm{m}^{2}}{\mathrm{~A}^{2}} \mathrm{~K} \cdot \mathrm{j}_{\text {macro }}^{2}+(298.9 \pm 0.7) \mathrm{K}$. Note that the current-dependence of Eq. (3.30) is due to temperature-independent reasons, namely the 
dependence on the sample width and the applied bias voltage $V_{\text {Bias }}$. We now fitted two models to the data points, the temperature-independent case

$$
\rho_{\text {Wrinkle }}(T)=\rho_{0} \cdot l_{\text {eff }}
$$

and a model for the temperature-dependent behavior as observed on the $\mathrm{ML}$ sheets

$$
\rho_{\text {Wrinkle }}(T)=\left[\rho_{0}+\rho_{A}(T)+\rho_{B}(T)\right] \cdot l_{\text {eff }}
$$

Here, we also introduced the effective length $I_{\text {eff }}$ that corresponds to the length of a graphene channel that would yield the same resistance as the defect. This concept has been used previously to describe localized defects $[61,210]$ and is introduced here to relate the defect resistance $\rho_{\text {Wrinkle }}([\Omega \mathrm{m}])$ to the sheet resistance $\rho_{\mathrm{ML}}([\Omega])$. We use $\mathrm{I}_{\text {eff }}=0.15 \mu \mathrm{m}$ so that the temperature-independent resistance $\rho_{0}$ is close to the measured sheet resistance $\rho_{\mathrm{ML}} \approx 350 \Omega$. In both models we use $\rho_{0}$ as the only fitting parameter. For the temperature-dependent case we use the

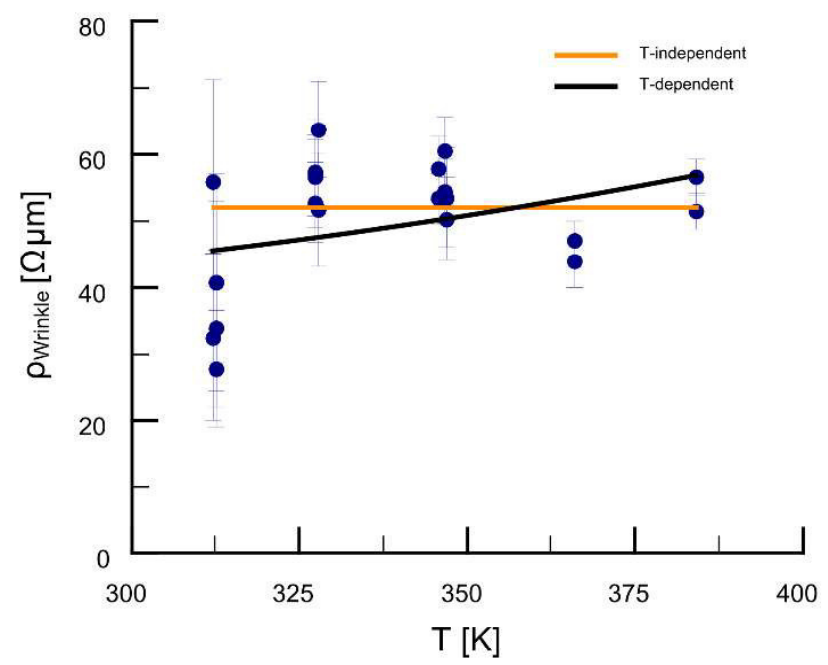

Figure 3.22: Step resistance $\rho_{\text {Wrinkle }}$ of the folded graphene wrinkle shown in Figure 3.17 as a function of temperature $\mathrm{T}$. Orange line indicates the fit to the temperature-independent model. The black line corresponds to the temperature-dependent model with an increase in resistance as observed on the graphene sheets.

same values for the parameters $K_{1}, K_{2}$ and $E_{0}$ as for the $M L$ sheet resistance $\rho_{M L}$ (see Supplementary Information, subsection 3.3.3.2). Note that the influence of the temperature-dependent part can be scaled by other choices of $\mathrm{I}_{\text {eff. }}$. The choices made here assume the same relative increase in the given temperature-range as for the monolayer sheet resistance $\rho_{\mathrm{ML}}$. This would for example be the case, if the transport through the wrinkle is simply given by the elongated path in case of no interlayer tunneling (see Supplementary Information, subsection 3.3.3.3). The best 
fits to the data for the T-independent case and for the T-dependent case are shown in Figure 3.22 as orange and black line, respectively.

While both lines seem to describe the data equally well, a more detailed statistical analysis reveals the differences in the quality of the fits. By comparing the chisquared

$$
\chi^{2}=\sum_{\mathrm{i}=1}^{\mathrm{N}}\left(\frac{\rho_{\mathrm{i}}-\rho\left(\mathrm{T}_{\mathrm{i}}\right)}{\sigma_{\mathrm{i}}}\right)
$$

with the error $\sigma_{\rho_{\text {Wrinkle }}}$ of the wrinkle defect resistance we find a $30 \%$ higher value for the T-dependent model than for the T-independent case.

$$
\frac{X_{\text {T-dependent }}^{2}}{X_{T \text {-independent }}^{2}}=130 \%
$$

In fact, this is even more pronounced when we exclude the data points at $\mathrm{T}=$ $312 \mathrm{~K}$. As can be seen from Figure $3.17 \mathrm{~d}$ the total voltage drop $\Delta \mathrm{V}$ is very small and close to the noise limit. This leads to high errors, since $\sigma_{\rho_{\text {Wrinkle }}}=\frac{\sigma_{\Delta V}}{\Delta V} \cdot \rho$. While these points cannot be described well by any of the two models, they are the only ones motivating a positive slope as required for the T-dependent model. Thus, excluding the data at $\mathrm{T}=312 \mathrm{~K}$ from the statistical analysis even leads to

$$
\frac{X_{\text {T-dependent }}^{2}}{X_{\text {T-independent }}^{2}}=193 \%
$$

so almost twice as high for the T-dependent case. This even holds for different choices for the effective length $\mathrm{I}_{\text {eff. }}$. As discussed above, this effectively scales the influence of the temperature-dependent part of the resistance. In Figure 3.23a we show the dependence of $\chi^{2}$ on $\mathrm{l}_{\text {eff }}$ for both models. As can be seen, for all choices of $\mathrm{I}_{\text {eff }}$, we find that $\chi^{2}$ is higher for the temperature-dependent model. For small values of $\mathrm{I}_{\text {eff }}$, the temperature-dependent data converges to the temperature-independent case. However, in this limit the temperature-dependence is so small that it hardly differs from the independent model as can be seen in Figure 3.23b.

Consequently, if the wrinkle resistance $\rho_{\text {Wrinkle }}$ was temperature-dependent, our data suggests that this dependence would be smaller than the ML sheet resistance, while the best fit is obtained with a temperature-independent model. Thus, we hypothesize that the step resistance $\rho_{\text {Wrinkle }}$ at the folded graphene wrinkle does not change with temperature. 
3.3 Local transport measurements in graphene on $\mathrm{SiO} 2$ using Kelvin probe force
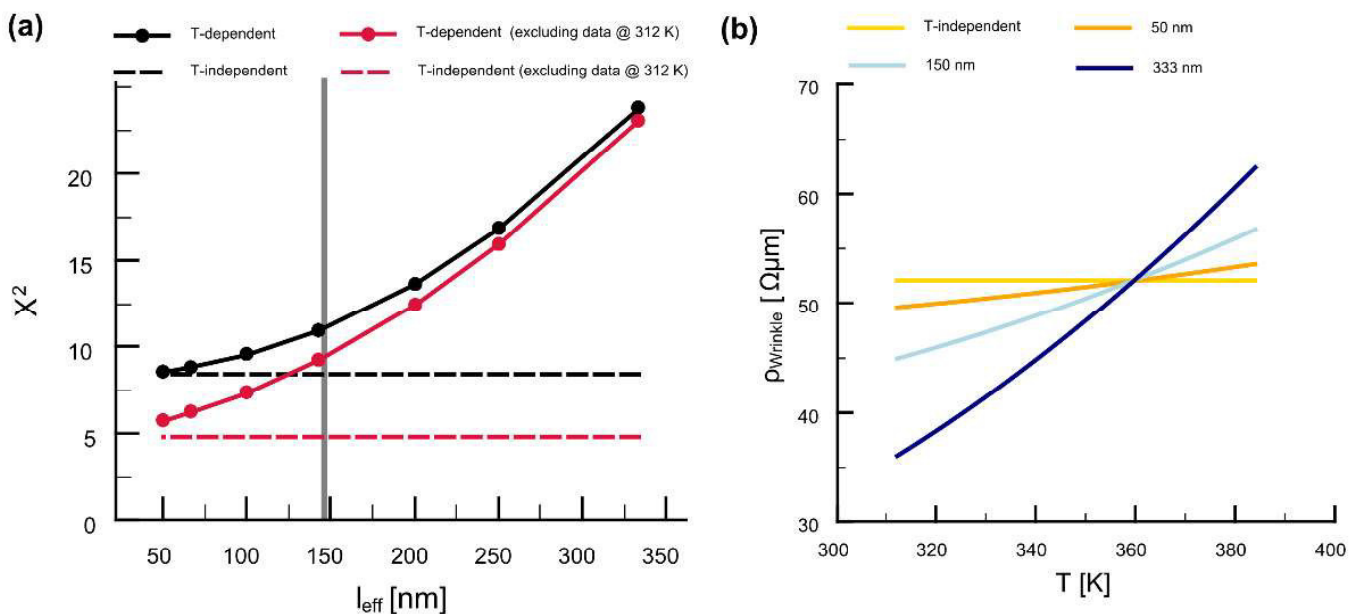

Figure 3.23: (a) Chi-squared $\chi^{2}$ as a function of effective length $\mathrm{I}_{\text {eff }}$ for all data points (black) and for excluding the lowest data points at $\mathrm{T}=312 \mathrm{~K}$ (red). The continuous (dashed) lines show the results for the temperature-dependent (temperature-independent) model. Grey line indicates the choice of $\mathrm{l}_{\text {eff }}$ for the same temperature-dependence as for the $M L$ sheet resistances. (b) Wrinkle resistance $\rho_{\text {Wrinkle }}$ as a function of temperature $T$ for different values of $l_{\text {eff }}$. 


\subsection{Magnetotransport on the nano scale}

Philip Willke, Thomas Kotzott, Thomas Pruschke, Martin Wenderoth

Nature Communications 8: 15283, April 2017

DOI: $10.1038 /$ ncomms 15283

Transport experiments in strong magnetic fields show a variety of fascinating phenomena like the quantum Hall effect, weak localization or the giant magnetoresistance. Often they originate from the atomic-scale structure inaccessible to macroscopic magnetotransport experiments. To connect spatial information with transport properties various advanced scanning probe methods have been developed. Capable of ultimate spatial resolution, scanning tunneling potentiometry has been used to determine the resistance of atomic-scale defects such as steps and interfaces. In this letter we combine this technique with magnetic fields and thus transfer magnetotransport experiments to the atomic scale. Monitoring the local voltage drop in epitaxial graphene, we show how the magnetic field controls the electric field components. We find that scattering processes at localized defects are independent of the strong magnetic field while monolayer and bilayer graphene sheets show a locally varying conductivity and charge carrier concentration differing from the macroscopic average.

\subsubsection{Introduction}

To elucidate the scattering mechanisms of electrons in a solid the dependence of the electrical resistance on an external magnetic field, the so-called magnetoresistance (MR), has been a versatile tool connecting theoretical considerations with macroscopic transport measurements[31,78]. The origin of the particular MR is often found on a nanometer scale. Examples are manifold, ranging from the giant magnetoresistance[5, 62], weak localization[78] or simply structural disorder[35, $89,205]$. Here, the MR reflects the scattering mechanisms induced by atomic-scale defects and nanostructures or the presence of local variations in conductivity and mobility.

Magnetotransport measurements in graphene have been of particular interest since its discovery due to exceptional transport properties including a remarkably high mobility[56, 135]. The latter is naturally limited by defects as a source of scattering[30, 42, 80, 87, 89, 108, 181, 201, 203, 210]. Due to the small spatial extent their influence on transport is often difficult to access. Dissecting different sources of scattering or detecting inhomogeneities in doping or conductivity becomes thus a challenging task. Large scale transport measurements combined with spatially 
resolving techniques such as electron microscopy helped to disentangle delocalized and localized contributions of electron transport [89, 181]. Using STP in previous studies on graphene allowed conclusions on the underlying scattering mechanism at localized defects by the magnitude[30, 80] or the position[201] of the voltage drop.

Here, we introduce a high magnetic field low-temperature STP setup to extract the (magneto-)resistance of localized defects. We can show that the resistances of all examined defects are independent of magnetic field strongly differing from pristine sample regions. For monolayer graphene (MLG) and bilayer graphene (BLG) sheets we find local variations in both conductivity and charge carrier concentration that also differ from the macroscopic mean values of the sample. We are able to derive a consistent picture of magnetotransport down to the atomic scale that could up to now only be discussed by theory[169]. Since none of the transport mechanisms are exclusively dedicated to graphene's unique electronic structure, our findings can be generalized to transport in other systems.

\subsubsection{Results}

\subsubsection{Magnetic-Field Scanning Tunneling Potentiometry}

Figure 3.24a depicts the experimental low-temperature (6 K) STP setup[41, 201]. A transverse magnetic field up to $6 \mathrm{~T}$ perpendicular to the current direction can be applied. In a first step the MR of one of our samples [epitaxial graphene on $\mathrm{SiC}(0001)$ [46, 201]] can be determined macroscopically in-situ (Figure 3.24b) showing mainly a positive quadratic slope $R(B) \propto(\mu B)^{2}$ with small corrections at low fields due to weak localization[81, 200] (For all samples see Supplementary Figure 3.28). In Figure 3.24c we show a typical sample region of MLG and BLG. In our experiment the strong quadratic MR is a consequence of the device geometry[106] $L \sim W$. In combination with the Lorentz-force induced by the magnetic field, electrons get deflected (See Supplementary Note 1 [subsection 3.4.5.3]) leading to a non-trivial potential drop as demonstrated in Figure 3.24d. Here, resistor network simulations are shown as a function of magnetic field $B$. These have been obtained by using finite-element method simulations (See Supplementary Note 12 [subsection 3.4.5.3-3.4.5.4], Supplementary Figure 3.28-Figure 3.31). Being below the quantum limit $\mu B \leq 1$ this pronounced MR in Figure $3.24 \mathrm{~b}$ is especially visible in devices with MR geometry which we particularly chose here to detect small changes in potential and consequently in resistance on a local scale (See Supplementary Note 1 [subsection 3.4.5.3], Supplementary Figure 3.30). Whereas the magnetic field dictates the overall shape of the potential drop in the sample on a large scale, local defects and variations in mobility lead to inhomogeneous voltage drops. This is demonstrated in Figure 3.24e for the sample surface area shown in Figure 3.24c in the range between $-6 \mathrm{~T}$ to $6 \mathrm{~T}$. As can be seen for the zero-field 
case the voltage drop is localized at steps, wrinkles, and interfaces in contrast to the MLG and BLG sheets[80, 201]. For finite magnetic field, the change in direction of the voltage drop agrees with the macroscopic direction.

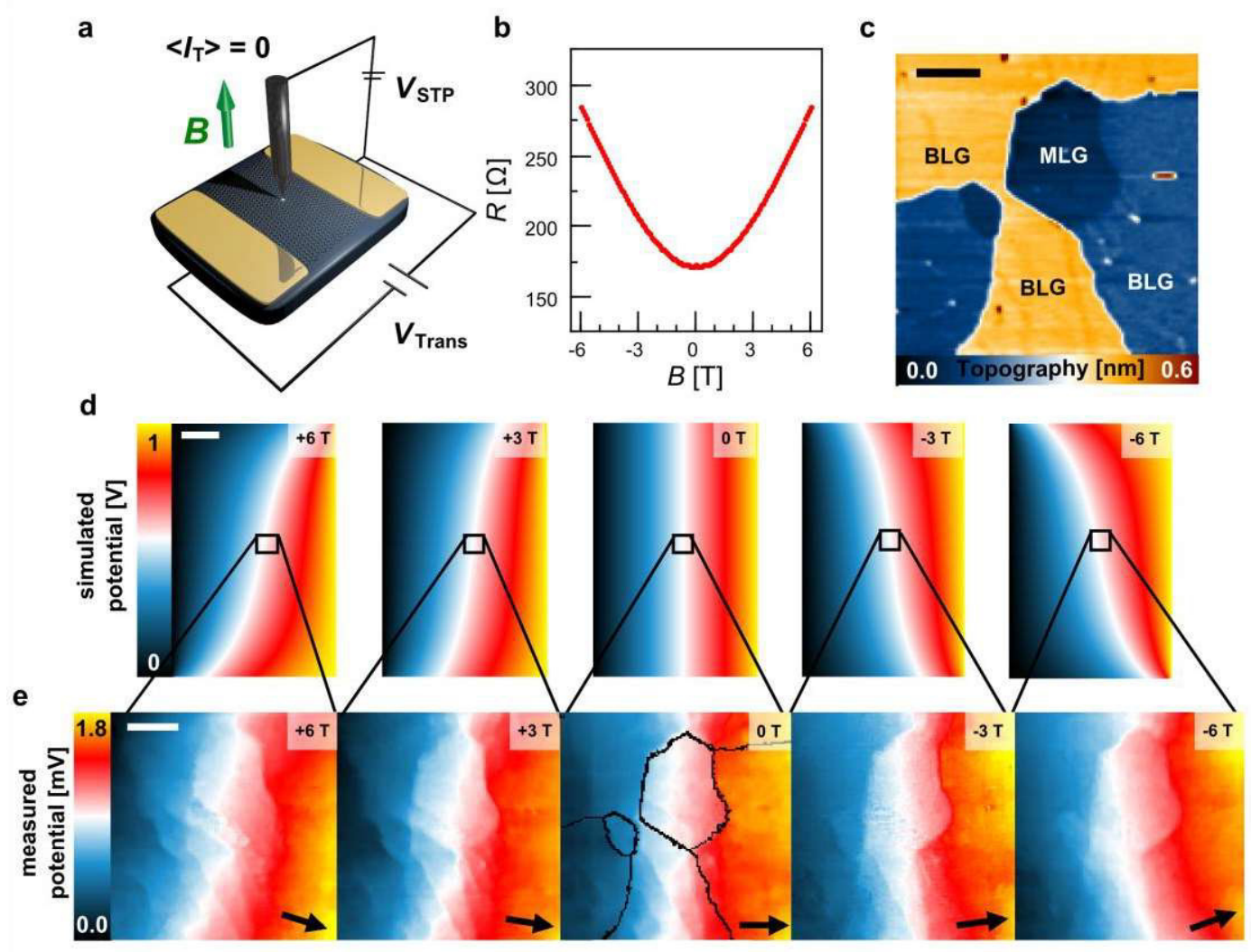

Figure 3.24: Scanning tunneling potentiometry with applied magnetic field. (a) Schematic of the setup: large samples of epitaxial-grown graphene on $\mathrm{SiC}(0001)$ consisting of monolayer and bilayer graphene are contacted in 2-terminal geometry. The voltage $\left.V_{\text {STP }}(\mathrm{x}, \mathrm{y})\right|_{I_{\mathrm{T}}=0}$ necessary to compensate a net tunnel current $I_{\mathrm{T}}$ is recorded and mapped. It represents the voltage drop along the sample induced by the cross voltage $V_{\text {Trans. }}$. A transverse magnetic field up to $6 \mathrm{~T}$ can additionally be applied. (b) Macroscopic resistance $R(B)$ of one of the investigated samples as a function of magnetic field $B$. (c) Topography of a typical sample area showing a monolayer graphene island surrounded by bilayer graphene $\left(440 \times 440 \mathrm{~nm}^{2}\right.$, scale bar $\left.100 \mathrm{~nm}, I_{\top}=0.2 \mathrm{nA} / V_{\text {Bias }}=-50 \mathrm{mV}\right)$. (d) Magnetic field dependent potential landscape for one of the samples in (b) for a cross voltage $V_{\text {Trans }}=1 \mathrm{~V}$ obtained by resistor network simulations $(1.6 \mathrm{~mm} \times 1.1 \mathrm{~mm}$, scale bar $250 \mu \mathrm{m}$, see Supplementary Note 1-2 [subsection 3.4.5.3-3.4.5.4] and Supplementary Figure 3.28-Figure 3.31). (e) Local potential maps for different magnetic fields $(-6 \mathrm{~T} /-3 \mathrm{~T} / 0 \mathrm{~T} /+3 \mathrm{~T} /+6 \mathrm{~T})$ for the sample region in (c) with black lines indicating the steps, arrows the direction of predominate electron flow [Scale bar $100 \mathrm{~nm}, V_{\text {Trans }}=4.2 \mathrm{~V} / j_{\text {macro }}(0 \mathrm{~T})=15.3 \mathrm{~A} / \mathrm{m}$ ]. 


\subsubsection{Local Hall-effect measurements}

For increasing magnetic field the voltage is also dropping in $y$-direction, visualizing the emerging Hall field on the nano scale. In Figure 3.25a and Figure 3.25b we show the spatially averaged voltage drops across the sample area in Figure $3.24 \mathrm{e}$ in $x$ - and $y$-direction, respectively. For the voltage drop in $x$-direction (Figure 3.25a) we find it to be monotonous with additional contributions from the local defects. Their influence diminishes with increasing magnetic field. In contrast, the voltage drop in $y$-direction (Figure 3.25b) changes in sign and increases with $B$. Moreover, it is inhomogeneous due to the presence of the defects, predominately due to the centered monolayer island in this data set.

a

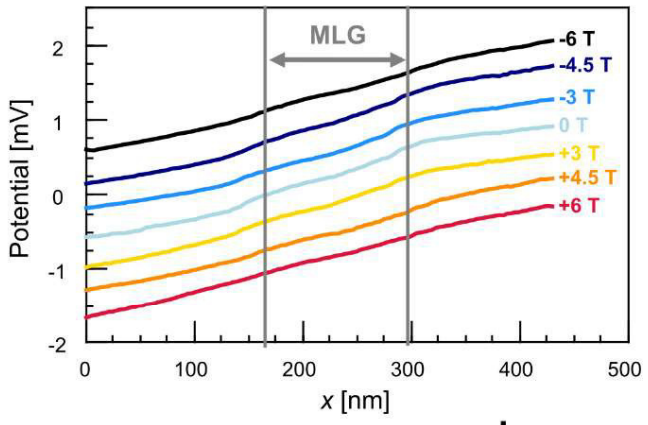

C

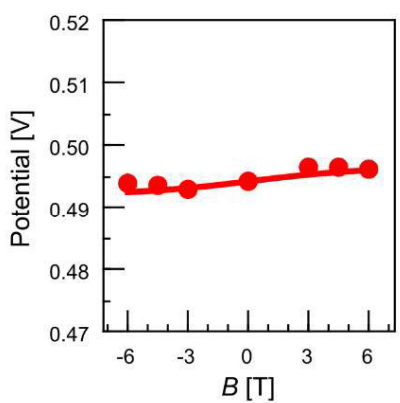

d

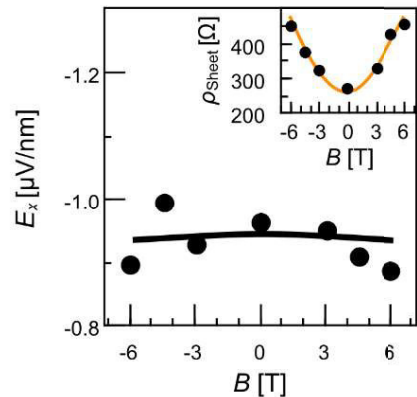

b

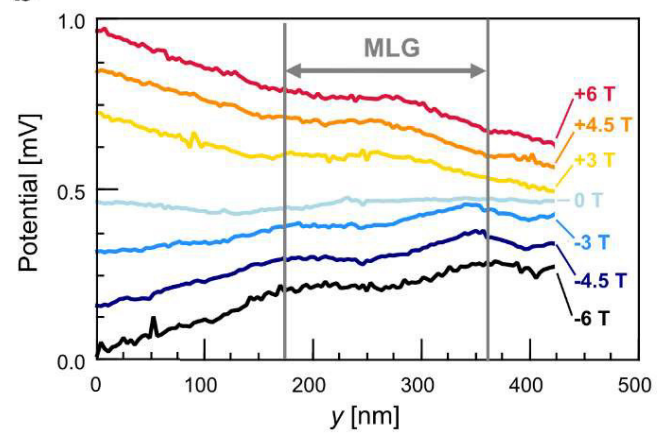

e



Figure 3.25: Hall measurements on the atomic scale. (a) Potential across the sample region shown in Figure 3.24c in $x$-direction (averaged in $y$-direction) and (b) in $y$-direction (averaged in $x$-direction). Lines have been shifted relative to each other. (c) Average potential as a function of magnetic field. (d)-(e) Electric field components $E_{x}$ and $E_{y}$ as a function of magnetic field $B$ derived by linear fits from the data in (a) and (b). The lines are the results of the macroscopic finite element simulation as shown in Figure 3.24d at the precise position of the measurement (See Supplementary Note 3 [subsection 3.4.5.5], Supplementary Figure 3.32). For comparison, the experimental electric fields are normalized to $V_{\text {Trans }}$ $=1 \mathrm{~V}$. The inset shows $E_{x} / j_{x}$ with the macroscopic sheet resistance (yellow, taken from Figure 3.24b)

Comparing the absolute value of the local potential at the position of the tip as well as the average field components $E_{x}$ and $E_{y}$ as a function of $B$ (Figure 3.25c-e) to the values of the macroscopic resistor network simulations (See Supplementary 


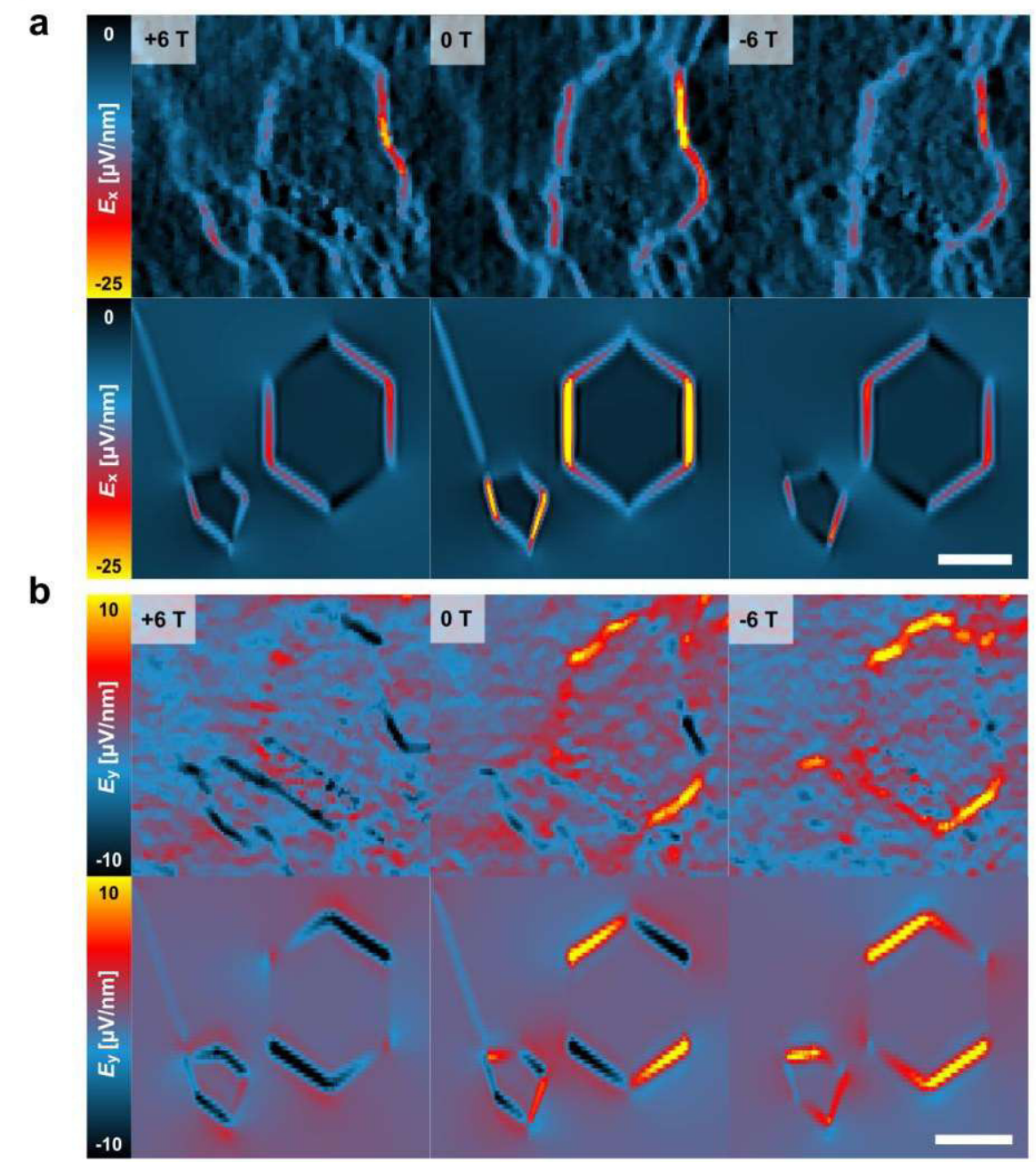

Figure 3.26: Local change of electric fields. (a) Electric field component $E_{x}$ for the area surrounding the monolayer graphene island derived from the potential maps in Figure $3.24 \mathrm{e}\left(-6 \mathrm{~T} / 0 \mathrm{~T} /+6 \mathrm{~T}\right.$, area: $\left.350 \times 350 \mathrm{~nm}^{2}\right)$. The lower row shows finite element method simulations (See Supplementary Note 5 [subsection 3.4.5.7]) of the area with simple geometries for the ML/BL-interfaces and the wrinkle on the left. (b) Analysis of the electric field component $E_{y}$ analogous to (a).

Note 1 [subsection 3.4.5.3], Supplementary Figure 3.28-Figure 3.30) allows to quantitatively analyze the Hall field. In the simulations, the experimental macroscopic MR-curves shown in Figure 3.24b are fitted yielding an average (macroscopic) conductivity $\langle\sigma\rangle$ and charge carrier concentration $\left\langle n_{e}\right\rangle$ (Fitted values see Supplementary Table 3.5). These are denoted as averages here, since they contain mixed contributions from monolayer and bilayer areas as well as the influence of local defects (for $\langle\sigma\rangle$ ). The respective potential and electric fields for these averaged $\langle\sigma\rangle$ and $\left\langle n_{e}\right\rangle$ are also shown in Figure 3.25c-e. Both experimental data and simulations are in excellent agreement. Consequently, despite the local inhomogeneities, the macroscopic average for $\langle\sigma\rangle$ and $\left\langle n_{e}\right\rangle$ is restored on a scale of approximately $500 \mathrm{~nm}$, i.e. when averaged over a larger scale of defects and single 
MLG/ BLG areas. Note that the electric field and current density components can strongly vary across the sample (See Supplementary Note 2 [subsection 3.4.5.4], Supplementary Figure 3.31). It is therefore necessary to know the position of the measurement which we can precisely derive from the measured potential curve $V(B)$ in Figure 3.25c (See Supplementary Note 3 [subsection 3.4.5.5], Supplementary Figure 3.32).

From the data points in Figure 3.25e, we are able to determine the (local) charge carrier concentration $n_{e} \propto j_{x}(x, y, B) \cdot B / E_{y}(x, y, B)$, for which we find $n_{e}=$ $(1.32 \pm 0.12) \cdot 10^{13} \mathrm{~cm}^{-2}$ (Evaluation see Supplementary Note 4 [subsection 3.4.5.6]).
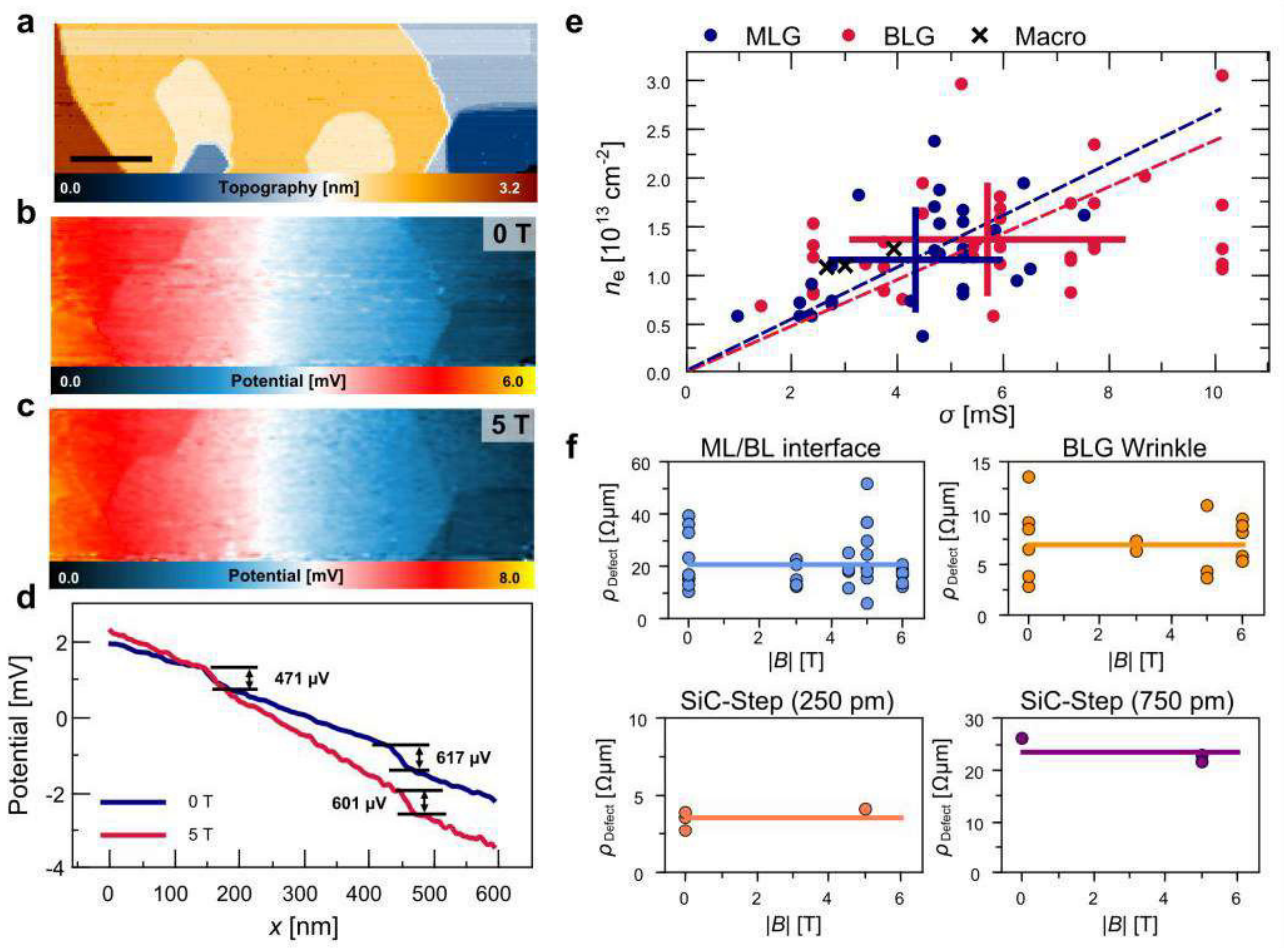

Figure 3.27: Magnetic field dependence of the resistance for graphene and defects.

(a) Topography of a microscopic section showing monolayer and bilayer graphene areas in addition to SiC-substrate steps and monolayer-bilayer-interfaces (Scale bar $100 \mathrm{~nm}$ ). (b) Corresponding potential map $V_{\text {STP }}$ for $0 \mathrm{~T}$ and (c) for $5 \mathrm{~T}$. Both maps have been rescaled to the same current density $j=17.7 \mathrm{Am}^{-1}\left(I_{\mathrm{T}}=0.3 \mathrm{nA} / V_{\text {Bias }}=50 \mathrm{mV}\right)$. Except for the difference in scale, both maps show a similar voltage drop. (d) Sections of the transport map. The voltage drop at the step/interface positions corresponding to the defect resistances is similar for both curves. The slope of the lines corresponding to the sheet resistances show a clear dependence on the magnetic field. (e) Conductivity $\sigma$ and charge carrier concentration $n_{e}$ derived for MLG (blue) and BLG (red) sheets from the change in voltage drop shown in d (See Supplementary Note 6 [subsection 3.4.5.8]). Additionally, the macroscopic values obtained from the MR curves in Figure 3.24b are plotted (black, See Supplementary Table 3.5). The solid lines show the standard deviation $\Delta n$ and $\Delta \sigma$ for both MLG and BLG. Dashed lines are a guide to the eye with the slope of the inverse MLG/BLG mobility $(e \mu)^{-1}$. (f) Resistances of all localized defects and their change with magnetic field. The lines indicate the $\mathrm{B}$-independent average. 


\subsubsection{Control and monitoring of the electric fields}

The additional influence of the magnetic field can nicely be pronounced by spatially resolved maps of the electric field components $E_{x}$ and $E_{y}$ in

Figure 3.26a and

Figure 3.26b, respectively. It shows how the current flow around the defect can be controlled by the magnetic field while being read out by STP. For comparison we added resistor network simulations of the area reduced to its major structural changes, the two MLG areas and a wrinkle on the left. For the magnetic field dependence we assumed the simplest model including a quadratic change with $B$ for the MLG/BLG sheets and $B$-independent defects (See Supplementary Note 5 [subsection 3.4.5.7], Supplementary Figure 3.34).

$\begin{array}{cccc} & \boldsymbol{\sigma}(\Delta \boldsymbol{\sigma})[\mathbf{m S}] & \mathbf{n}_{\boldsymbol{e}}\left(\Delta \mathbf{n}_{\boldsymbol{e}}\right)\left[\mathbf{1 0}^{\mathbf{1 3}} \mathbf{c m}^{-\mathbf{2}}\right] & \boldsymbol{\mu}\left[\mathbf{m}^{\mathbf{2}} \mathbf{V}^{\mathbf{- 1}} \mathbf{s}^{\mathbf{- 1}}\right] \\ \text { Macro } & 2.65-3.94 & 1.09-1.25 & 0.155-0.197 \\ \text { MLG } & 4.3 \pm 0.4(1.6) & 1.15 \pm 0.10(0.51) & 0.234 \pm 0.027 \\ \text { BLG } & 5.7 \pm 0.5(2.6) & 1.36 \pm 0.10(0.55) & 0.261 \pm 0.027\end{array}$

Table 3.3: Results for the conductivity $\sigma$, charge carrier concentration $n_{e}$ and mobility $\mu$ for macroscopic averaged measurements (See also Supplementary Table 3.5) as well as monolayer and bilayer graphene with their respective standard deviations $\Delta \sigma, \Delta n$.

$\begin{array}{cccc} & \boldsymbol{\rho}_{\text {Defect }}(\mathbf{0} \mathbf{T})[\Omega \mu \mathbf{m}] & \boldsymbol{\rho}_{\text {Defect }}(>\mathbf{0} \mathbf{T})[\boldsymbol{\Omega} \mathbf{m}] & \boldsymbol{\rho}_{\text {Defect }}[\boldsymbol{\Omega} \boldsymbol{\mathbf { m }}] \\ \begin{array}{c}\text { ML/BL-in- } \\ \text { terface }\end{array} & 22.5 \pm 11.7 & 19.7 \pm 10.1 & 20.6 \pm 7.5 \\ \text { Wrinkle } & 7.0 \pm 3.2 & 6.7 \pm 2.6 & 6.9 \pm 2.1 \\ \text { SiC-Step } & 3.4 & 4.1 & 3.6 \\ (250 \text { pm) } & & & \\ \text { SiC-Step } & 25.9 & 22.2 & 23.4 \\ (750 \text { pm) } & & & \end{array}$

Table 3.4: Results for defect resistance $\rho_{\text {Defect }}$ for the different types of defects in SiCgraphene and the change in magnetic field. While the first column shows the values for $B=0 \mathrm{~T}$, the second one averaged over all data points with applied magnetic field. The last column yields the total average. No errors for the SiC-substrate steps are given due to the small number of data points.

\subsubsection{Local magnetic field dependence of defects and sheets}

For now we found that on the nano scale $<500 \mathrm{~nm}$ the voltage drop becomes inhomogeneous due to defects and subsequently different current paths lead to large deviations for the electric field in $x$ - and $y$-direction. Thus, in the following the magnetic field dependence of the single contributions of graphene MLG/BLG sheets 
and defects to the resistance is evaluated (Figure 3.27). For the topography (Figure 3.27a) and potential maps acquired at different B fields (Figure 3.27b and Figure $3.27 \mathrm{c}$ ) we show an averaged section in Figure $3.27 \mathrm{~d}$. Here, the electric field $E_{x}$ increases on the MLG/BLG areas for the $5 \mathrm{~T}$ case compared to that at $0 \mathrm{~T}$. Since for a constant current density $j$ this increased electric field $E_{x}$ corresponds to a higher resistance (higher voltage drop per unit area), this qualitatively reflects the positive quadratic MR found in Figure $3.24 \mathrm{~b}$. For a quantitative evaluation of the change in $E_{x}$, an analysis taking into account the exact position on the sample is needed (See Supplementary Note 2 and 3 [subsection 3.4.5.4, 3.4.5.5], Supplementary Figure 3.31 and Figure 3.32). The local sheet conductivity $\sigma=j / E_{x}$ can be extracted from the electric field $E_{x}$ for $0 \mathrm{~T}$, its magnetic field-dependence gives access to the local charge carrier concentration $n_{\mathrm{e}}$ (Detailed discussion on evaluation see Supplementary Note 6 [subsection 3.4.5.8], Supplementary Figure 3.35 and Figure 3.36). Both quantities are shown in Figure 3.27e evaluated for a large number of sheets and datasets. We find a large spread of values for both sheet conductivity $\sigma$ and charge carrier concentration $n_{e}$ up to a factor of 10 indicating local inhomogeneities (standard deviation $\Delta \sigma$ and $\Delta n_{e}$ indicated in Figure 3.27e, see also Table 3.3).

In contrast to the MLG/BLG areas, the voltage drop $\Delta V$ of the two localized defects in Figure 3.27d and thus their defect resistances $\rho_{\text {Defect }}=\Delta V / j$ remains constant for different $B$-fields. In Figure 3.27f we show the defect resistances as a function of magnetic field for all extended defects in our epitaxial graphene sample, e.g. $\mathrm{ML} / \mathrm{BL}$-interfaces, wrinkles on BLG and substrate steps. Apparently, for all defect types the defect resistance remains constant effectively leading to a vanishing contribution at high fields (Figure 3.25a/Figure 3.27d), since the resistance contribution of the sheets in contrast still increases here (Figure 3.24b). Table 3.3 and Table 3.4 summarize the results for the sheets and the defect resistances, respectively.

\subsubsection{Discussion}

The charge carrier concentration $n_{e}=(1.32 \pm 0.12) \cdot 10^{13} \mathrm{~cm}^{-2}$ extracted from the local Hall measurements fits perfectly with the macroscopic value for bilayer graphene[137] and results from scanning tunneling spectroscopy (STS) (See Supplementary Note 4 [subsection 3.4.5.6], Supplementary Figure 3.33). While the latter also allows to extract $n_{e}$ our new method based on local voltage probes keeps the advantage that no a priori knowledge on the electronic structure is needed. Moreover, detection of smaller doping becomes additionally difficult in STS due to the presence of the pseudo-gap for graphene[201]. Despite the good agreement, the Hall field in Figure 3.25b clearly shows local deviations manifested in a non-linear voltage drop. This is attributed to variations in mobility and charge carrier density 
as well as defects, since they determine the local current density resulting in a severely changed Hall field on a scale $<500 \mathrm{~nm}$.

In addition, the resistor network simulations are also able to reproduce well the changes in electric field components with applied B-field on a local scale in

Figure 3.26, reflecting the change in electron flow around the center MLG island. Given that this is a classical model neglecting quantum mechanical effects as e.g. weak localization[81, 201] or Klein Tunneling[86] and only takes into account the main structural features this is quite remarkable. It demonstrates how using the magnetic field the direction of electron flow can be controlled on a nano scale.

For the MLG and BLG sheets the conductivity $\sigma$ given in Table 3.3 is higher than macroscopically observed, which obviously stems from the fact that the macroscopic conductivity still contains the influence of steps and interfaces. For the three samples studied in the framework of this work, a decrease in defect concentration showed consequently a higher macroscopic conductivity (Supplementary Figure 3.37, Supplementary Table 3.5). The MLG sheet resistance shown in Figure 3.27e and Table 3.3 agrees with previous transport measurements using Hall bars[82] and STP measurements[80, 201]. The average values of $n_{e}$ for MLG and BLG are in excellent agreement with spatially averaged values from ARPES[137]. Mobilities are as high as reported for defect-free graphene areas grown under Argon-atmosphere[46]. Thus, the transport properties of the UHV-grown samples are as good as the highest reported values on $\mathrm{SiC}$ when excluding the contribution of the defects. Moreover, the proportional trend $n_{e} \propto \sigma$ (dashed lines in Figure 3.27e) suggests that local variations in $\sigma$ are governed by local variations in $n_{e}$. This can be caused by the graphene buffer layer as well as stacking faults in bilayer graphene[69, 89]. Especially the graphene buffer layer can affect both $\sigma$ by local scattering potentials as well as $n_{e}$ by local changes in doping[157]. (See Supplementary Note 4 [subsection 3.4.5.6], Supplementary Figure 3.33). In addition, the large standard deviation for both $n_{e}$ and $\sigma$ suggests that the inhomogeneity of the buffer layer leads to a spread of local resistance. This was previously observed in STP measurements without magnetic field[42]. The sheet resistance increases by a factor of 2 when going from low temperatures $(4 \mathrm{~K})$ to room temperature[46, 82] and is almost constant in our samples at low temperatures $(<30 \mathrm{~K})[201]$. Therefore, it is likely that the interaction with the buffer layer is still lowering the conductivity compared to graphene on other substrates[25, 34]. The conductivity for BLG is slightly higher than for MLG. It is not simply given by twice the value of MLG, since only one bilayer band is populated at these doping concentration[137]. Additionally a lower doping in the upper layer and decreasing influence of the buffer layer lead to the conductivity given in Table 3.3. (Further discussion on the local conductivity see Supplementary Note 7 [subsection 3.4.5.9]). 
The constant MR for the localized defects allows to draw conclusions on the underlying scattering mechanisms. A decrease in doping caused by detachment from the substrate present for $\mathrm{SiC}$-steps and wrinkles has been previously suggested to explain the voltage drop without magnetic field[108]. This model needs to be extended, since also a graphene sheet with a different carrier density would show a $\mathrm{B}^{2}$-dependence. Instead a change in doping can be described as a potential barrier from a quantum mechanical point of view. Indeed for the transmission $T$ through a magnetic potential barrier based on wave function matching the MR remains constant, since the wave vector components $k_{x} / k_{y}$ barely change for barriers with a small extent (See Supplementary Note 8 [subsection 3.4.5.10], Supplementary Figure 3.38). For the $\mathrm{ML} / \mathrm{BL}$-interface the scattering due to wave function mismatch[80] and interlayer tunneling[201] has been discussed as the main contribution in absence of a magnetic field. Also these scattering mechanisms do not change significantly with magnetic field explaining the same behavior observed for $\mathrm{ML} / \mathrm{BL}$-interfaces. Though a variety of magnetic properties of this interface has been discussed including interface states and interface Landau-levels[95, 150], circulating edge states[105], they do not influence the resistance of this defect. Additionally, an angle-dependent transmission[86, 126] inevitably induced by the magnetic field does not play are role for the defects and their resistance.

Combining magnetotransport measurements with scanning probe methods opens a new path to tackle a wide range of transport phenomena on the atomic scale. For studies on a mesoscopic scale we suggest that this method can easily be implanted in an atomic force microscope setup using Kelvin probe force microscopy[203]. We here demonstrate for the first time the different roles of localized defects and pristine sample areas for the build-up of a classical quadratic MR. In the past, the MR in highly inhomogeneous systems[35, 205] including bilayer graphene[89] has been investigated intensively leading even to a linear MR in case of sufficient disorder. To test the existing theories[90,141] magnetotransport-STP will be an excellent tool, while the results of this work already demonstrate how different structural contributions change the local and macroscopic magnetic-field dependence. In addition magnetic tunnel junctions [5, 62], quantum Hall physics in graphene[82] as well as weak localization phenomena[201, 210] are future candidate systems bearing magnetoresistive effects on the nano scale.

\subsubsection{Methods}

\subsubsection{Sample Preparation}

Samples with epitaxial mono- and bilayer graphene are prepared by thermal decomposition of $\mathrm{n}$-doped $6 \mathrm{H}$-SiC(0001)[46] at $\mathrm{T}=1400-1600^{\circ} \mathrm{C}$ under ultra-high vacuum (UHV, $10^{-10} \mathrm{mbar}$ ). The samples $(2 \mathrm{~mm} \times 7 \mathrm{~mm}$ ) are electrically contacted exsitu with gold contacts of $100 \mathrm{~nm}$ thickness by thermal evaporation through a 
shadow mask. After reinsertion into the UHV chamber the samples are heated up to $350^{\circ} \mathrm{C}$ for 30 minutes to eliminate surface contaminations before they are transferred in-situ to a homebuilt low-temperature scanning tunneling microscope (STM). All measurements were performed at $6 \mathrm{~K}$ sample temperature.

\subsubsection{Scanning Probe Measurements}

Scanning Tunneling Potentiometry (STP) measurements are taken at every image point by adjusting the electrochemical potential (ECP) at the tip at fixed tip-sample distance. For STP the applied bias voltage is switched off while only the transport potential across the sample remains. The potential at the tip is adjusted in a way that the tunneling current $I_{\mathrm{T}}=0$. Subsequently, the voltage $\left.V_{\mathrm{STP}}(x, y)\right|_{I_{\mathrm{T}}=0}$ necessary to compensate the net tunnel current is recorded (See Figure 3.24a). This voltage $V_{\mathrm{STP}}=\frac{\mu_{\mathrm{ECP}}}{e}$ has been referred to as the local ECP, which is here inherently defined by the STP method[31, 41]. Thermovoltage contributions have been eliminated as described in Ref. [201]. The measurements are made at different values of the electron current in the sample plane, especially at zero and forward and reversed current as defined by the potential applied to the sample contacts. The details of our specific setup are published elsewhere[41]. A superconducting coil magnet implemented in the microscope was used to create a strong magnetic field at the position of the sample. Due to the high stability of the system the magnetic field can be changed while staying in tunneling contact between tip and sample. This allows us to take STP measurements at the same position as a function of the magnetic field.

\subsubsection{Data availability}

The MR curves, potential datasets and values for conductivity, charge carrier concentration and defect resistances are available from the authors.

\subsubsection{Acknowledgment}

This work was supported by the Deutsche Forschungsgemeinschaft (DFG) priority program 1459 Graphene. We thank A. Heinrich, K. Pierz and H. B. Weber for fruitful discussions and B. Spicher for expert technical assistance.

\subsubsection{Author contributions}

M.W. and P.W. planned the experiments; P.W. and T.K. carried out the experiments and the data analysis. P.W. wrote the manuscript; all authors discussed the results and commented on the manuscript.

\subsubsection{Competing financial interests}

The authors declare no competing financial interests. 


\subsubsection{Supplementary Information}

\subsubsection{Supplementary Figures}
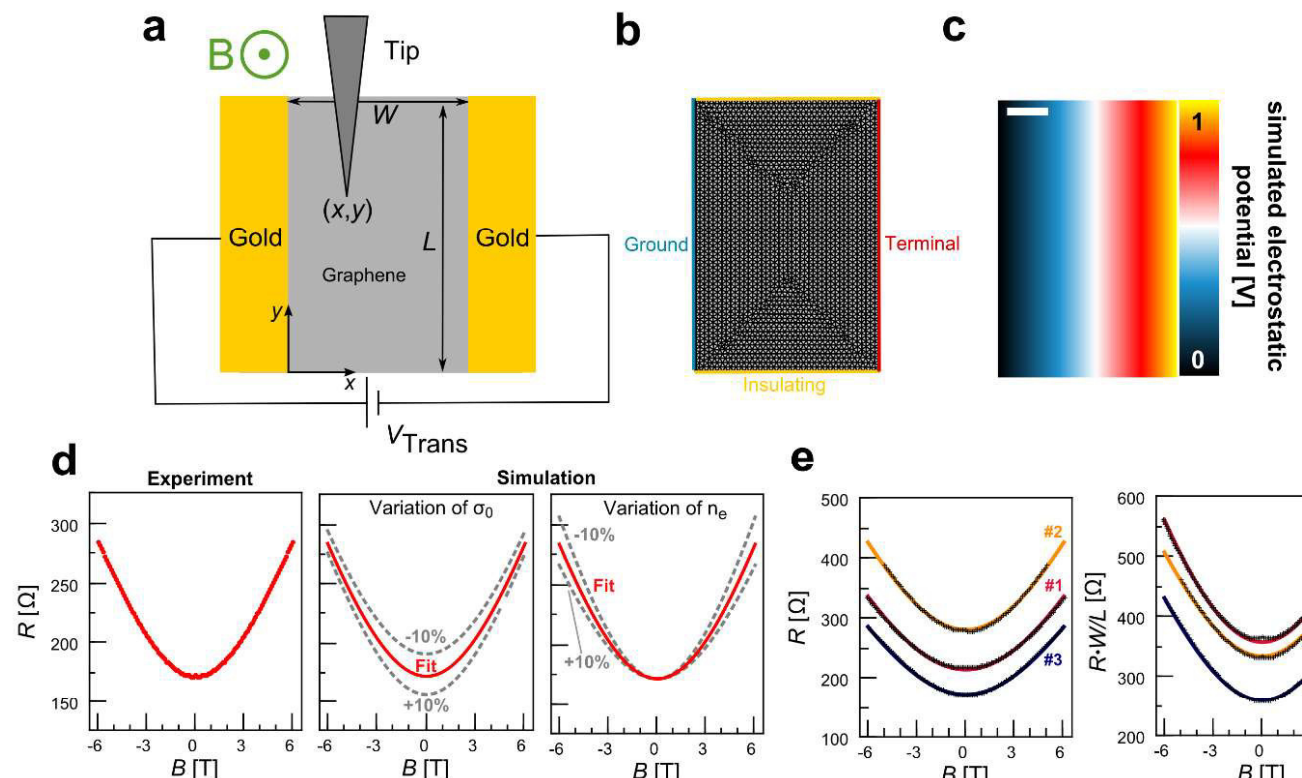

Figure 3.28: Finite element simulations and fitting of MR-curves. (a) The graphene sample of width $W$ and length $L$ is contacted in two-point geometry $\left(W \cdot L \approx 2-3 \mathrm{~mm}^{2}\right.$, see Supplementary Table 3.5). The coordinates $(x, y)$ as used in the text refer to the position of the tip. (b) Mesh grid and electrical boundary conditions. Black lines show the triangles used in the finite element simulations as initiated by COMSOL Multiphysics. The terminal is set to $1 \mathrm{~V}$. (c) Electrostatic potential obtained from the finite element simulation as shown in Figure 3.24d in the manuscript (Scale bar $250 \mu \mathrm{m}$ ). (d) Fitting of the experimental data to the finite element simulation. Left: Experimental MR for sample \#3 in Supplementary Table 3.5, which is also shown in Figure 3.24 in the manuscript. Middle: Fit of conductivity $\sigma_{0}$. Red line shows the best fit to the data with $\sigma_{0}=3.94 \mathrm{mS}$; Grey lines show a $10 \%$ deviation from this value $\left(n_{e}=1.25 \cdot 10^{13} \mathrm{~cm}^{-2}\right.$ for all curves). Right: Fit of the charge carrier concentration $n_{e}$. Red line shows the best fit to the data with $n_{e}=1.25 \cdot 10^{13} \mathrm{~cm}^{-2}$; Grey lines show a $10 \%$ deviation from this value ( $\sigma_{0}=3.94 \mathrm{mS}$ for all curves). (e) Total resistance $R$ for all samples investigated in this study and the normalized resistance $R \cdot W / L$ as a function of magnetic field $B$. Black dots are experimental data and colored lines are fits done as sketched in d. Sample numbers correspond to those shown in Supplementary Table 3.5 . 


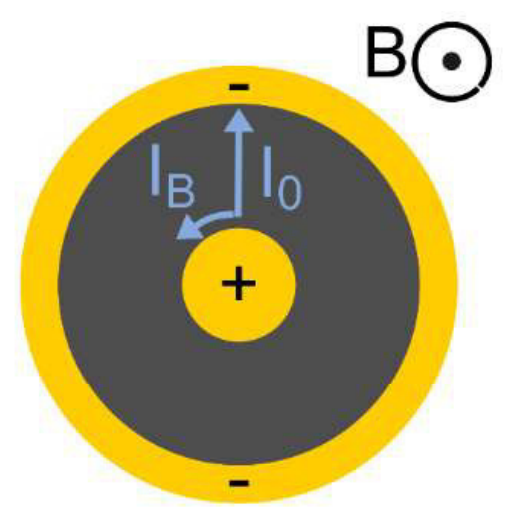

Figure 3.29: Corbino disk contact geometry. Contacts are given by an inner and an outer circle while a transverse (perpendicular) magnetic field $\mathrm{B}$ is applied. For increasing $B$ the current $I_{0}$ changes by the additional component $I_{B}$. Thus, the path an electron has to travel in the medium with resistivity $\rho_{0}$ increases leading to a positive MR.
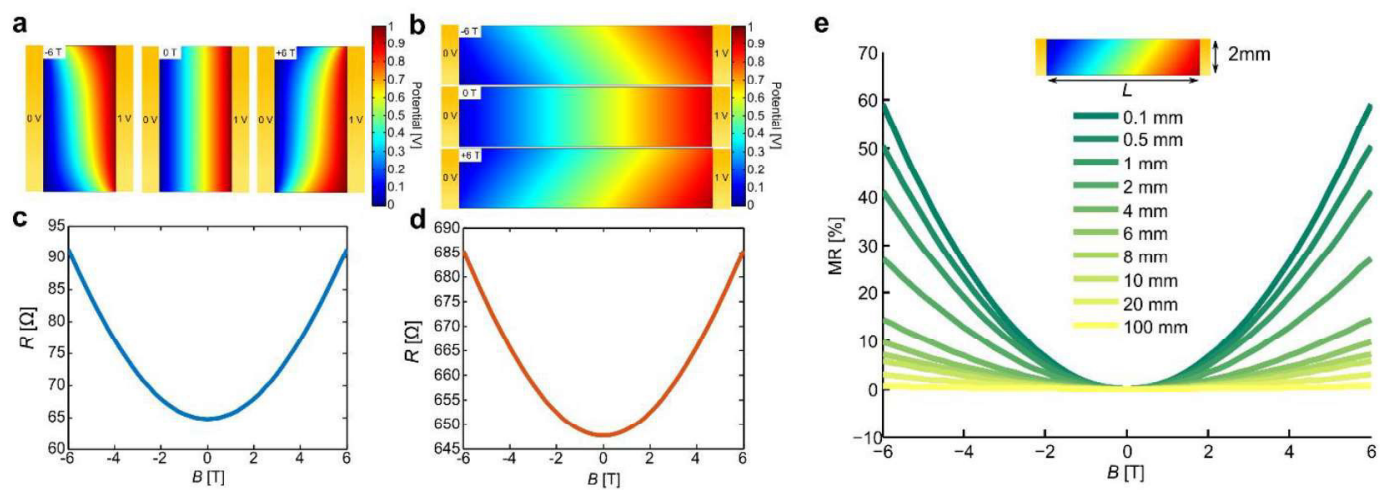

Figure 3.30: Potential distribution and Magnetoresistance for different contact geometries. (a) Sample in short-channel geometry (MR geometry) with low ratio $L / W=$ $1 \mathrm{~mm} / 2 \mathrm{~mm}=0.5$. (b) Long sample (Hall-geometry) with high ratio $L / W=10 \mathrm{~mm} / 2 \mathrm{~mm}=$ 5. (c) $R(B)$ for the sample geometry in a. (d) $R(B)$ for the sample geometry in c. (e) Magnetoresistance for different aspect ratios. Color-coded plots are simulated for different length $L$ of the sample while the width $W=2 \mathrm{~mm}$ is held constant. Thus, the aspect ratio $L / W$ changes. Accordingly, for increasing aspect ratio the MR decreases. (Simulation parameters: $\sigma_{0}=7.84 \mathrm{mS} / n_{e}=1.23 \cdot 10^{13} \mathrm{~cm}^{-2}$ ) 
a

b

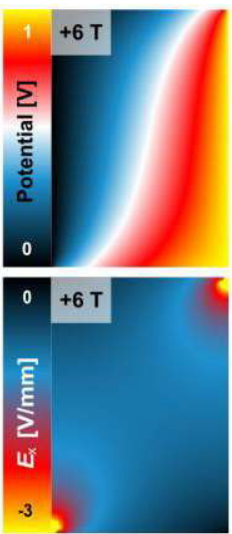

C

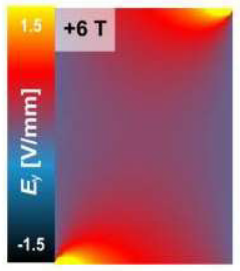

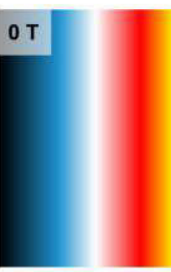
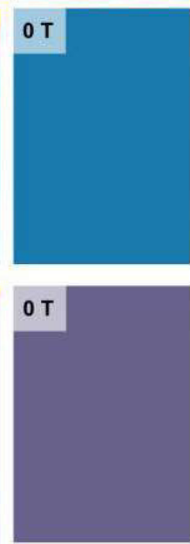
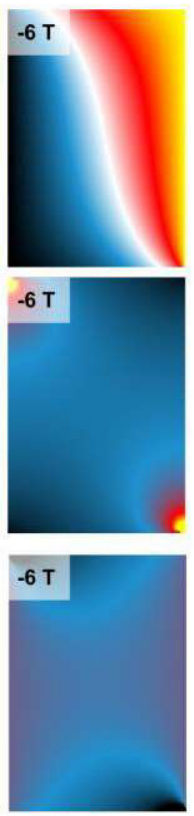

d



e
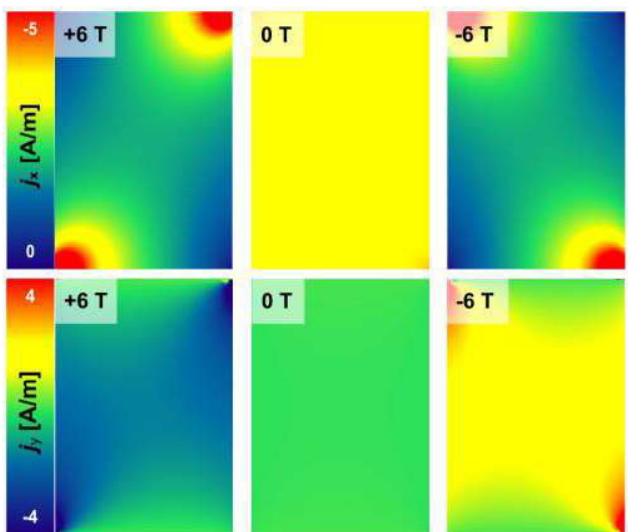

$-6 \mathrm{~T}$

$500 \mu \mathrm{m}$

Figure 3.31: Macroscopic FEM simulations. (a) Potential distribution as shown in Figure $3.24 \mathrm{~d}$ in the manuscript. (b) Electric field component $E_{x}$. (c) Electric field component $E_{y \cdot}$. (d) Current density component $j_{x}$ (e) Current density component $j_{y}$.

a
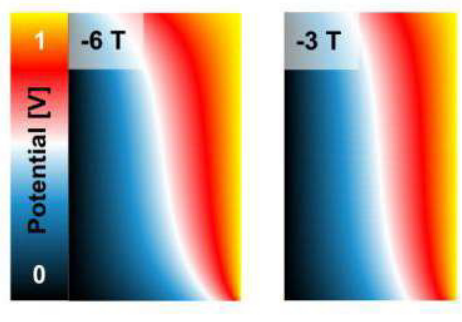

b

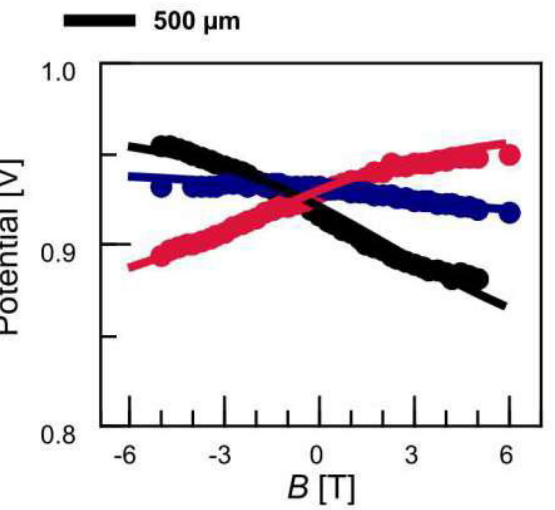

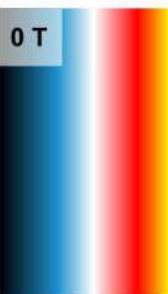
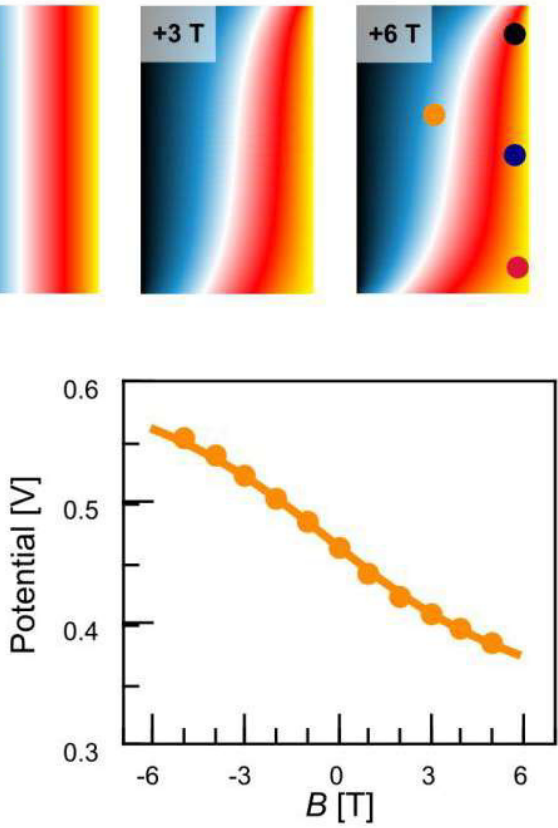

Figure 3.32: Positioning via magnetic field-dependent potential shifts. (a) Potential distribution as shown in Figure 3.24d in the manuscript, but for sample \#1 in Supplementary Table 3.5. (b) Experimentally measured change in potential at the positions indicated in (a) with the respective curves from the simulation (solid lines). These positions of the tip can be roughly determined by an optical access within a range of $\approx 100 \mu \mathrm{m}$. Within this experimentally determined range, the position-dependent, simulated $V(B)$ curves have been further fitted to yield the best agreement with the experimental $V(B)$. Thus, a very exact determination of the tip position is possible. 
a

b
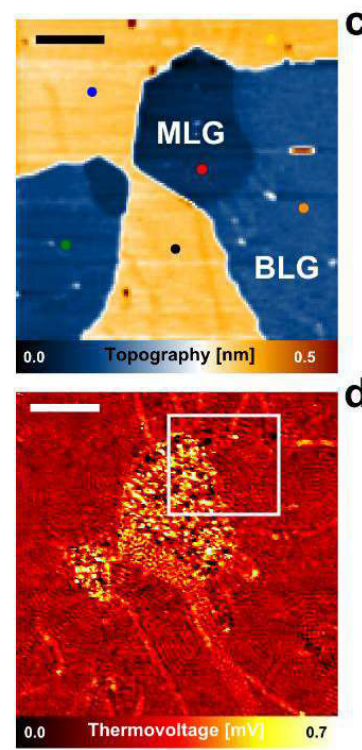
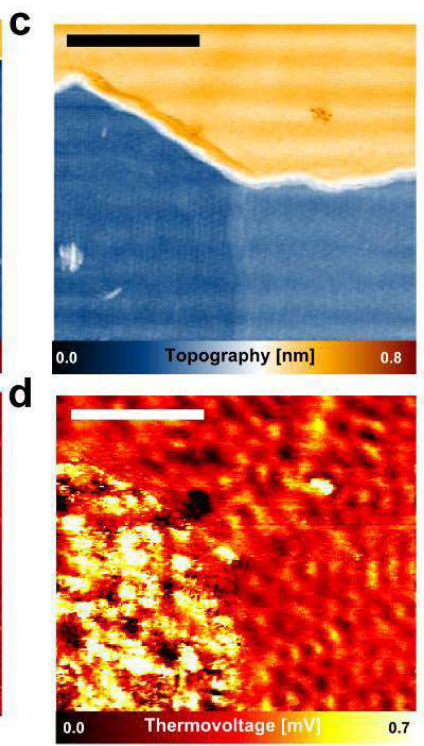

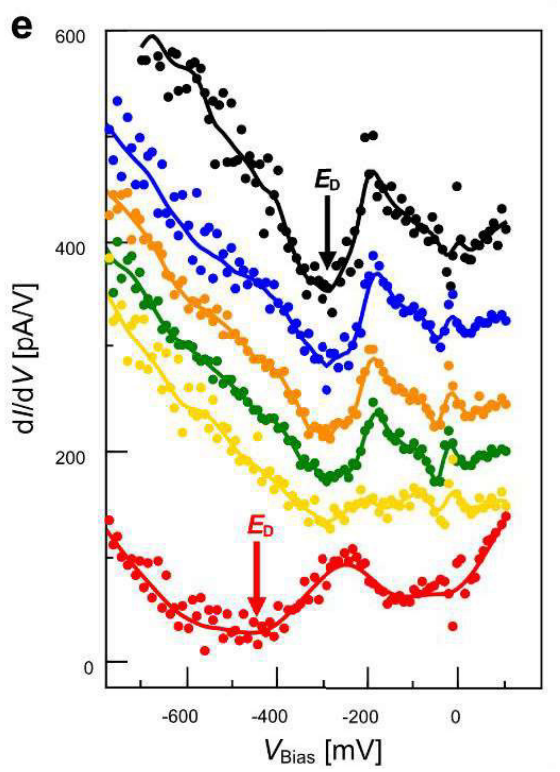

Figure 3.33: Scanning tunneling spectroscopy of monolayer and bilayer graphene. (a) Topography of the sample region in Figure $3.24 \mathrm{c}$ in the manuscript. (b) Thermovoltage map. (c-d) High resolution images of the upper right area in (a) and (b) [white square in b]. (e) Scanning tunneling spectroscopy taken at the points indicated in (a). While the red line is taken on MLG, all other spectra are taken on BLG areas. The arrows indicate the position of the Dirac point $E_{\mathrm{D}}$. 


\section{a}
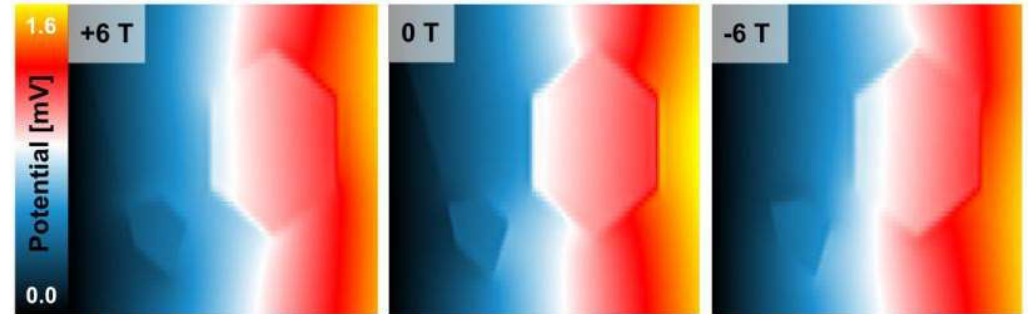

b
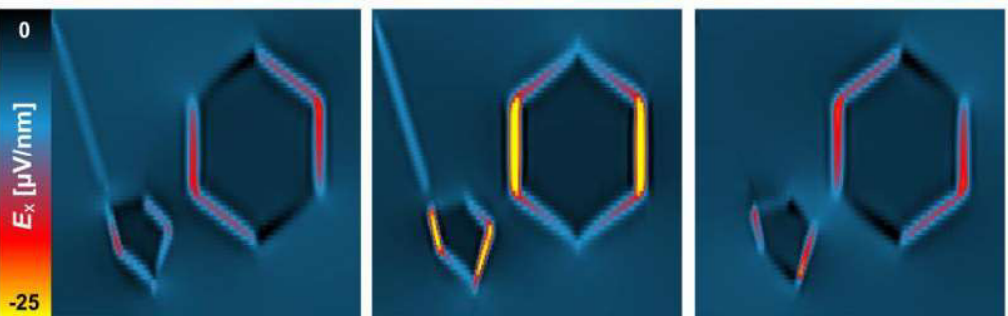

C
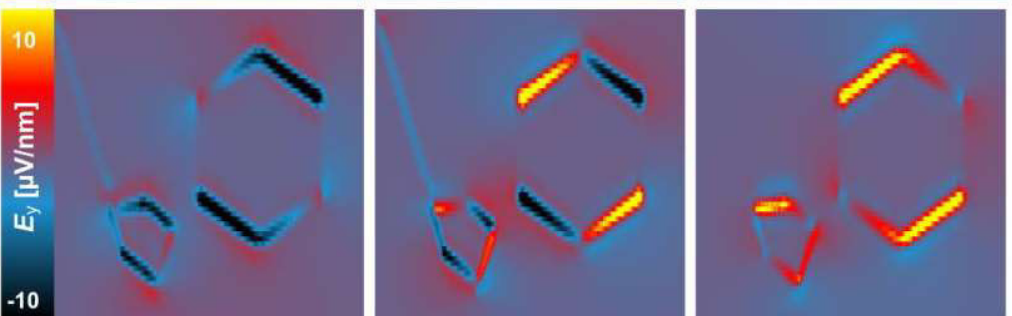

d
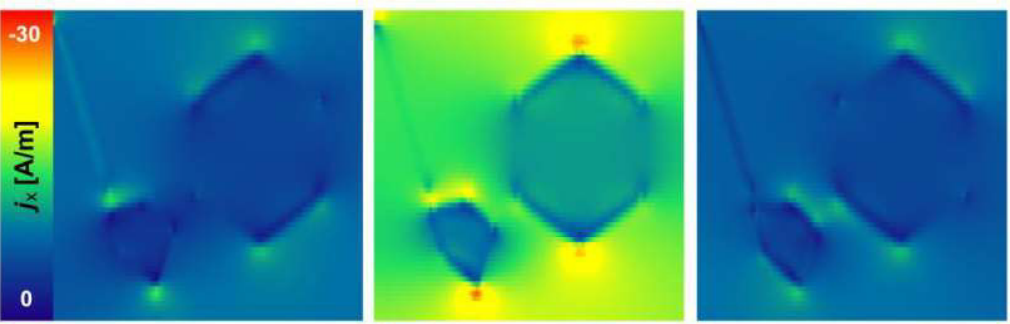

e
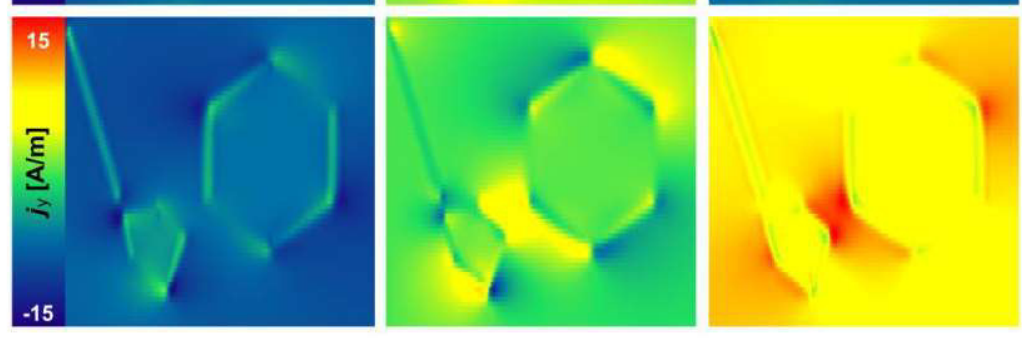

$100 \mathrm{~nm}$

Figure 3.34: FEM simulation for microscopic sample structure. Magnetic field dependent simulation for the sample topography in Figure $3.24 \mathrm{c}$ in the manuscript with (a) potential (b) electric field $E_{x}$ (c) electric field $E_{y}$ (d) current density $j_{x}$ and (e) current density $j_{y}$. 


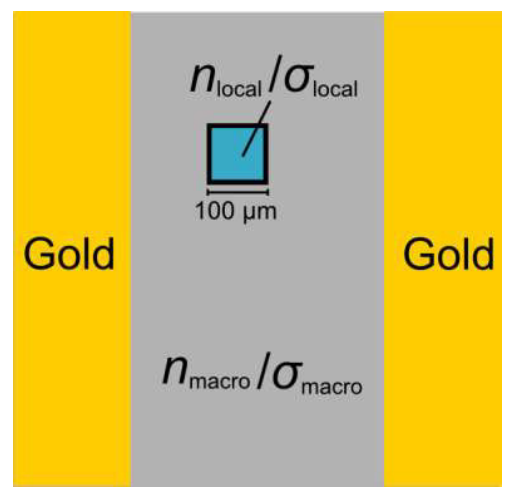

Figure 3.35: Geometry for FEM simulations to extract $\boldsymbol{\sigma}$ and $\boldsymbol{n}_{\boldsymbol{e}}$ locally. For each sample geometry the conductivity tensor uses the macroscopic (average) values of $\sigma$ and $n_{e}$ as shown in Supplementary Table 3.5 (grey area). Locally both quantities are varied in a region of $100 \mu \mathrm{m} \times 100 \mu \mathrm{m}$ (blue area). The position of that area fits that deduced from the potential analysis $V(B)$ for each dataset (see Supplementary Note 3 [subsection 3.4.5.5]). A small region (black area) of higher resistivity has been included to ensure continuity of current density in that sample region. 
a

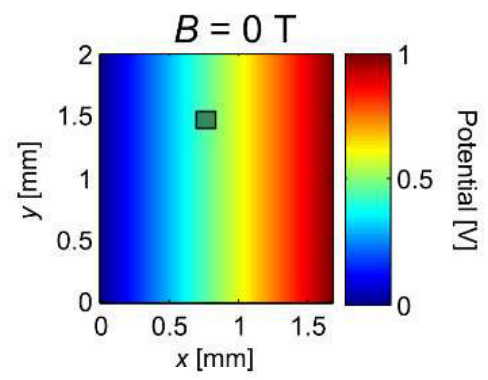

C

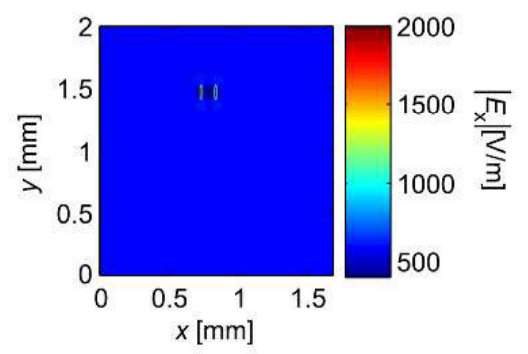

e

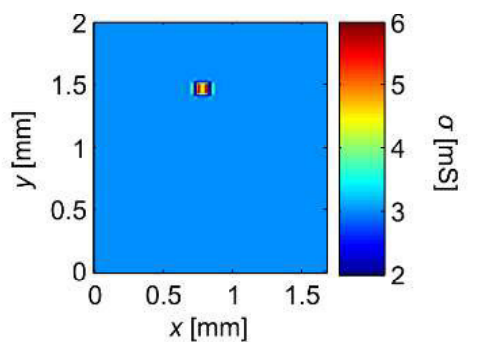

g

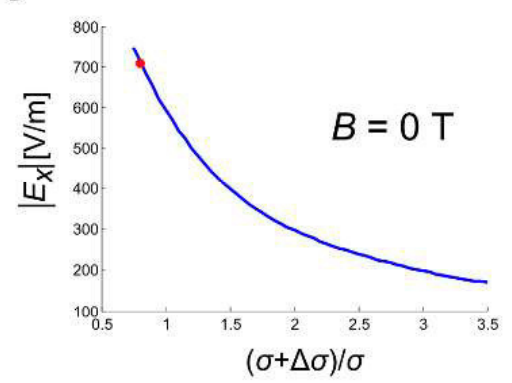

b

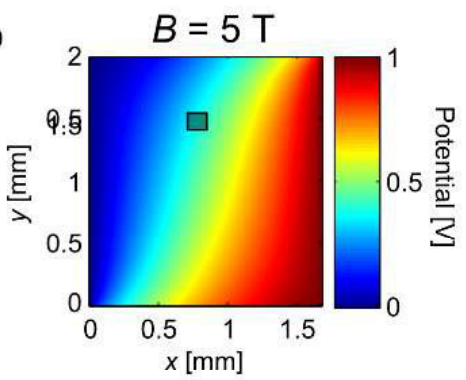

d

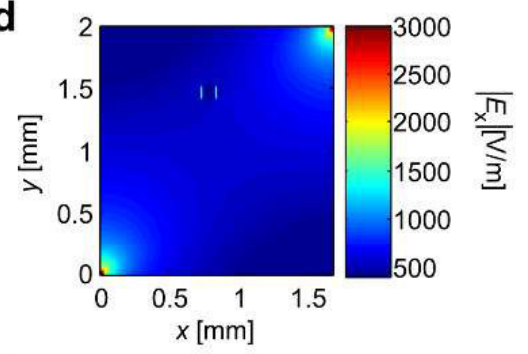

f

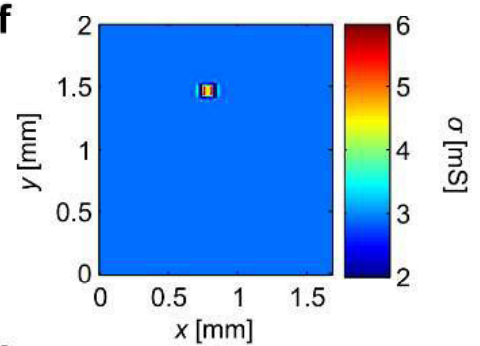

h

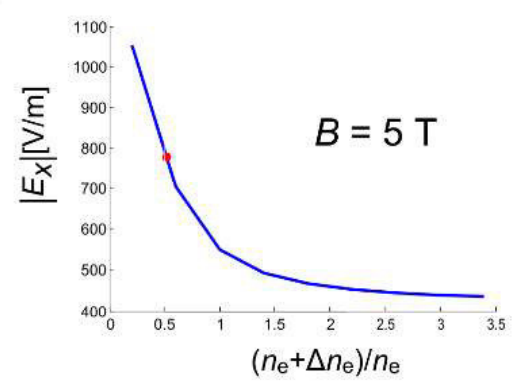

Figure 3.36: Extracting $\sigma$ and $n_{\boldsymbol{e}}$ from FEM simulations. Potential for (a) $0 \mathrm{~T}$ and (b) 5 $\mathrm{T}$. In the indicated area the conductivity $\sigma$ and the charge carrier concentration $n_{e}$ have been increased by $50 \%$. The corresponding electric field in $\mathrm{x}$-direction and the calculated local conductivity are shown in (c)-(d) and (e)-(f), respectively. (g) Fit of the electric field $E_{x}$ as a function of local conductivity $(\sigma+\Delta \sigma) / \sigma, 0 \mathrm{~T}$. (h) Fit of the electric field $\mathrm{E}_{\mathrm{x}}$ as function of local charge carrier concentration $\left(n_{e}+\Delta n_{e}\right) / n_{e},, 5 \mathrm{~T}$. 

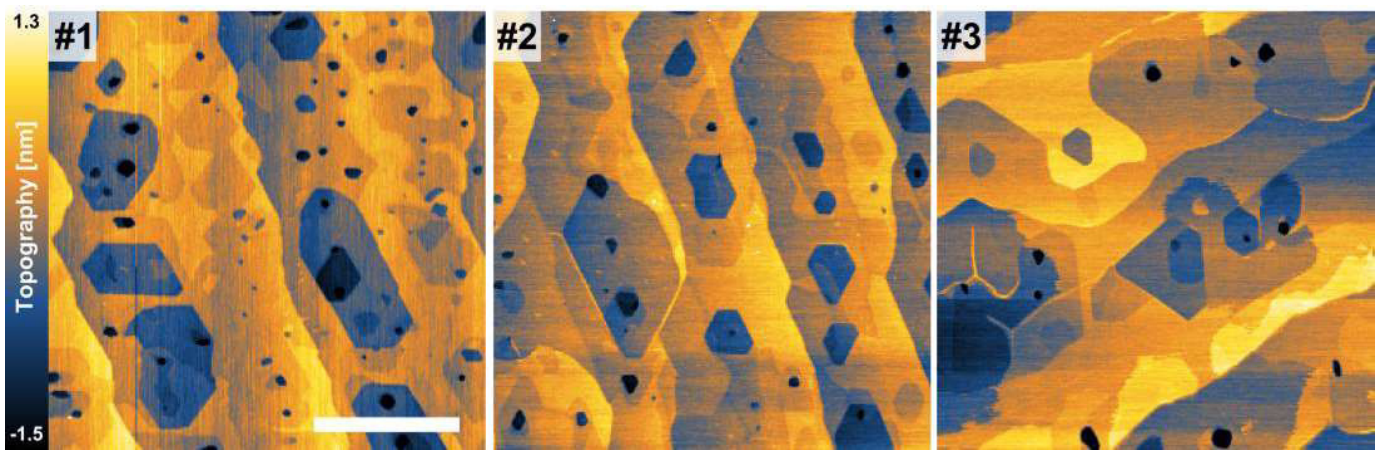

Figure 3.37: AFM topographies of all investigated samples. Sample No. is indicated in the upper left corner relating the images to the MR curves in Supplementary Figure 3.28e and to the values extracted in Supplementary Table 3.5 (Scale bar $1 \mu \mathrm{m}$ ). For all we find roughly an equal ratio of MLG/BLG-areas.

a

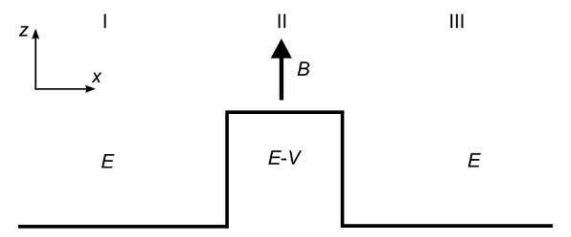

b

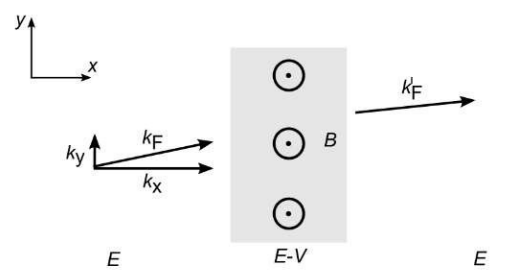

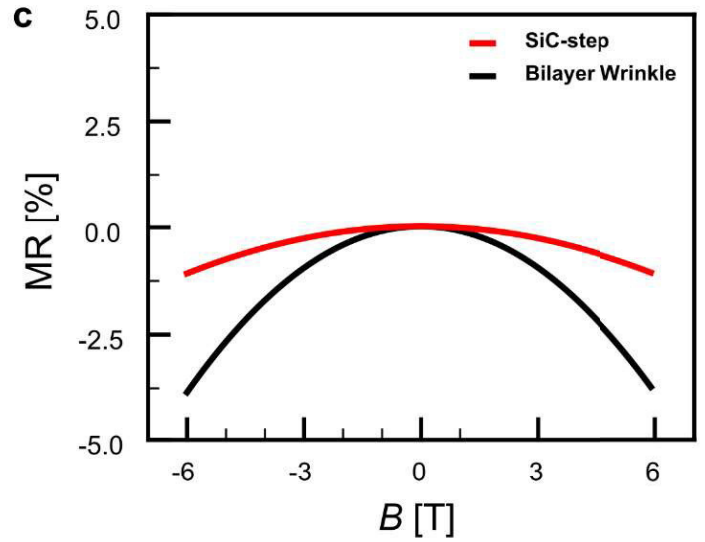

Figure 3.38 Transmission through a magnetic potential barrier. (a) Potential barrier with an additional transverse magnetic field inside. (b) Topview with components of the wave vector. (c) Theoretical magnetoresistance for monolayer SiC-steps and bilayer wrinkles as a function of the magnetic field. 


\subsubsection{Supplementary Tables}

$\begin{array}{ccccc}\begin{array}{c}\text { Sample } \\ \text { No. }\end{array} & \begin{array}{c}\boldsymbol{W} \times \boldsymbol{L} \\ {[\mathrm{mm} \times \mathrm{mm}]}\end{array} & \langle\boldsymbol{\sigma}\rangle[\mathbf{m S}] & \left\langle\boldsymbol{n}_{\boldsymbol{e}}\right\rangle\left[\mathbf{1 0}^{\mathbf{1 3}} \mathbf{c m}^{-\mathbf{2}}\right] & \langle\boldsymbol{\mu}\rangle\left[\mathbf{m}^{2} / \mathbf{V} \cdot \mathbf{s}\right] \\ \mathbf{1} & 2.1 \times 1.2 & 2.65 & 1.07 & 0.155 \\ \mathbf{2} & 2.0 \times 1.7 & 3.02 & 1.09 & 0.173 \\ \mathbf{3} & 1.6 \times 1.1 & 3.94 & 1.25 & 0.197\end{array}$

Table 3.5: Sample dimensions, microscopically averaged conductivity $\langle\sigma\rangle$, averaged charge carrier concentration $\left\langle n_{e}\right\rangle$ and averaged mobility $\langle\mu\rangle$ for the macroscopic measurements for the samples used in this study.

\subsubsection{Supplementary Note 1}

Finite element method simulations of the macroscopic MR curves:

\section{a) Derivation of the conductivity tensor}

For a transverse magnetic field $\mathbf{B}$ perpendicular to the current flow $\mathbf{j}=-e n_{e} \mathbf{v}_{\mathrm{D}}$ charge carriers get deflected by the additional Lorentz-force. Here, $v_{\mathrm{D}}$ is the drift velocity and $n_{e}$ is the charge carrier concentration. This can be written as[31]

$$
\frac{m}{\tau} \boldsymbol{v}_{\mathbf{D}}=-e\left[\boldsymbol{E}+\boldsymbol{v}_{\mathbf{D}} \times \boldsymbol{B}\right]
$$

with $m$ being the electron effective mass and $\tau$ the momentum relaxation time. Choosing $\boldsymbol{B}=(0,0, B)$ and solving for Ohm's law in the form $\boldsymbol{E}=\hat{\sigma}^{-1} \boldsymbol{j}$ in the limit of 2D transport yields[31]

$$
\begin{gathered}
\left(\begin{array}{c}
E_{x} \\
E_{y}
\end{array}\right)=\left[\begin{array}{cc}
m / e \tau & -B \\
B & m / e \tau
\end{array}\right]\left(\begin{array}{l}
v_{x} \\
v_{y}
\end{array}\right)=\sigma_{0}^{-1}\left[\begin{array}{cc}
1 & \mu B \\
-\mu B & 1
\end{array}\right]\left(\begin{array}{l}
j_{x} \\
j_{y}
\end{array}\right) \\
=\left[\begin{array}{cc}
\rho_{0} & -R_{H} B \\
R_{H} B & \rho_{0}
\end{array}\right]\left(\begin{array}{l}
j_{x} \\
j_{y}
\end{array}\right)
\end{gathered}
$$

Moreover, the Hall resistance $R_{H}=-1 / e n_{e}$ was introduced. The subscript for $\sigma_{0}=$ $e^{2} \tau n_{e} / m=1 / \rho_{0}$ indicates the zero-field conductivity/resistivity. Inverting the resistivity matrix yields

$$
\hat{\sigma}=\frac{\sigma_{0}}{1+(\mu B)^{2}}\left[\begin{array}{cc}
1 & -\mu B \\
\mu B & 1
\end{array}\right]
$$

where $\mu=\sigma_{0} / e \cdot n_{e}$.

\section{b) Resistor-Network simulation using COMSOL Multiphysics}

The resistor network simulations have been conducted using finite element method (FEM) simulations with COMSOL Multiphysics (Version 5.2). Using the AC/DC module, in particular the electric currents toolbox, a simple rectangular sample has 
been simulated in a 2D space dimension (Supplementary Figure 3.28a). Width $W$ and length $L$ were taken from the actual samples geometries. Two sides (top, bottom) were set as electric insulation while the contacts were set as ground and a terminal as sketched in Supplementary Figure 3.28b. In addition to avoid numerical errors of device resistance, the mesh size has been set to extra fine $(<40 \mu \mathrm{m}$ edge length, Supplementary Figure 3.28b). Thus, no changes with varying mesh size were found.

The magnetic field $B_{z}$ in z-direction has been included by a manual input of the conductivity tensor in Supplementary (3.36). Parametric sweeps for the $B_{z}$-field were done in the range between $-6 \mathrm{~T}$ to $+6 \mathrm{~T}$. For further reading a detailed introduction on the simulation of Hall devices using COMSOL is given in Ref. [149]. In addition to the resulting spatially resolved electrostatic potential (Supplementary Figure 3.28c) as well as electric field and current density components (See Supplementary Note 2 [subsection 3.4.5.4]), the total resistance $R(B)$ (magnetoresistance) of the device in Supplementary Figure 3.28a-c can be obtained from the simulation. To obtain the macroscopic (averaged over the whole sample) conductivity $\sigma_{0}$ and charge carrier concentration (CCC) $n_{e}$ the simulated $R(B)$ curves were fitted to the experimental ones. Supplementary Figure 3.28d shows the experimental MR curve for sample \#3 also shown in Figure 3.24 in the manuscript along with simulations for different values of $\sigma_{0}$ and $n_{e}$. For $B=0$ only $\sigma_{0}$ influences the total resistance and can thus be deduced (Supplementary Figure 3.28d, middle, this is also for rectangular samples simply given as $\left.\sigma_{0}=R(0)^{-1} \cdot L / W\right)$ ). In addition, for $B>0$ the $\operatorname{CCC} n_{e}$ changes the MR and can thus additionally be determined (Supplementary Figure 3.28d, right). In Supplementary Figure 3.28e, left fits to all samples of this study are shown. While the difference in offset and slope of the curves stems on the one hand from variations in width $W$ and length $L$ of the samples [See also discussion in Supplementary Note 1(c)] it is on the other hand also a result of different defect densities of steps and interfaces (influencing $\sigma_{0}$, see also Supplementary Figure 3.37) and different ratio between monolayer and bilayer graphene (influencing $\sigma_{0}$ and $n_{e}$ ). The fitted values for all samples are shown in Supplementary Table 3.5. For better comparison of the samples Supplementary Figure 3.28e, right shows the normalized resistance $R \cdot W / L$ which is the sheet resistance $\rho_{\text {sheet }}=1 / \sigma_{0}$ for $B=0 \mathrm{~T}$.

\section{c) Geometry-dependence of the magnitude of the magnetoresistance}

As discussed in the manuscript the geometry of a device has a crucial influence on the absolute value of the total resistance $R(B)$. A geometry-related increase of MR is nicely visualized in the geometry of a Corbino disk[32, 196] (Supplementary Figure 3.29) and it should not be confused with a B-field dependency of the diagonal element of the conductivity tensor. For no magnetic field, the electrons can 
directly flow from the inner to the outer contact, while for finite field they get deflected by an angular component. Due to the radial symmetry, they still move in the outside radial direction, but cannot travel the shortest current path. Therefore, more scattering events occur and $R(B)$ increases. Since for this geometry the electric field components do not change under the influence of a magnetic field, the change in MR[196]

$$
R(B)=R_{0} \cdot\left[1+(\mu B)^{2}\right]
$$

can directly be seen from the denominator of the prefactor in Supplementary Equation (3.36). Now, this geometric MR can be more or less pronounced depending on the sample geometry. For example, in a Hall geometry (e.g. Supplementary Figure $3.30 \mathrm{~b}$ ) the Hall field completely compensates the Lorentz force leading to $R(B) \approx R_{0}$.

Supplementary Figure 3.30 shows simulations for different sample geometries in case of no magnetic field as well as for $\pm 6 \mathrm{~T}$. In the spatially resolved potential images in Supplementary Figure 3.30a the contacts induce boundary conditions, since they are on a fixed potential and thus the equipotential lines in their vicinity are heavily bent for applied magnetic field $B$ : Electrons are deflected in the sample due to the Lorentz force and pile up on one side causing a voltage $V_{\mathrm{H}}$ in y-direction. In contrast to the Corbino disk the existence of a Hall field can partially compensate for the magnetic forces and thus the geometric MR. However, unless the aspect ratio of a Hall geometry is reached (Supplementary Figure $3.30 \mathrm{~b}$ ) the sample still shows a geometric MR as in Supplementary Equation (3.37) induced by the contact regions. Here, the deflection is not fully compensated as in the case of the Corbino disk making the contact regions responsible for the observed MR (An extended discussion on this issue is given in Ref. [90]). Therefore, short-channel samples with $L<W$ (Supplementary Figure 3.30a) are dominated by the contact regions and show a strong MR (Supplementary Figure 3.30c, relative increase $\approx 50 \%$ ). In contrast, for long thin geometries with $L>W$ shown in Supplementary Figure 3.30b (Hall-geometry) this contribution vanishes, since a constant electric field gradient $E_{y}$ in $y$-direction has been established here and electrons are no further deflected because $V_{H}$ compensates the Lorentz force. The respective MRcurve is shown in Supplementary Figure 3.30d with almost no dependence on magnetic field (obey different $y$-scale, relative increase $\approx 5 \%$ ). Supplementary Figure 3.30e quantifies this observation demonstrating that the magnitude of the observed MR is especially present for short samples and is vanishing in the limit of perfect Hall geometries.

In the framework of this study, the geometry was on purpose chosen to be shortchan-neled (Supplementary Figure 3.30a) instead of using a Hall-geometry (Supplementary Figure $3.30 \mathrm{~b}$ ). In this way, the magnetic-field independent behavior of 
the defect resistances was easier to distinguish from the change in electric fields on the sheets.

\subsubsection{Supplementary Note 2}

Inhomogeneity of local current densities and electric fields: In a rectangular shaped sample as shown in Supplementary Figure 3.28a the current density components $\mathrm{j}_{\mathrm{x}}$, $\mathrm{j}_{\mathrm{y}}$ as well as the electric field components $E_{x}, E_{y}$ cannot be assumed to be homogeneous under the influence of a magnetic field. This is demonstrated in Supplementary Figure 3.31 for the geometry of sample \#3 that is also shown in Figure 3.24-

Figure 3.26 in the manuscript. Especially at the corners of the sample the electric fields and current densities increase/decrease drastically. As a consequence the values in the center are also smaller than could be expected from e.g. simply calculating $j_{x}=V_{\text {Trans }} / W \cdot R(B)$ for the current density in $x$-direction, where $R(B)$ is the total resistance of the sample as a function of magnetic field $B$.

\subsubsection{Supplementary Note 3}

Determination of the tip position from potential measurements: The current density $\mathrm{j}$ is not uniform for an applied magnetic field (Supplementary Figure 3.32). However, since this value is needed to extract e.g. the defect resistance $\rho_{\text {Defect }}=$ $\Delta V / j_{x}$ we developed a method to determine the tip's position. Then the current density at this position is estimated by the resistor network simulations. In the experiment the approximate position of the tip can be obtained via an optical access. However, we determine the position of the tip on the sample more precisely by the change in potential $V(B)$ with magnetic field. While the potential varies only along the $x$-direction for $0 \mathrm{~T}$, it also changes in $y$-direction for a nonzero magnetic field. Therefore, the $x$-position of the tip on the sample can be determined with $B=0 \mathrm{~T}$ while its change with magnetic field determines the $y$-position. This is demonstrated for different positions of the tip on the sample (Supplementary Figure 3.32a) in Supplementary Figure 3.32b with excellent agreement between experiment and simulation. Thus, by comparing the potential as a function of magnetic field with the simulations the position of the tip can be extracted.

\subsubsection{Supplementary Note 4}

Charge carrier concentration on $M L$ and $B L$ graphene: From the Hall field shown in Figure 3.25b and evaluated in Figure 3.25d in the manuscript we can extract the charge carrier concentration. The electric field component $E_{y}$ in an arbitrarily rectangular sample is given by[79] 


$$
\begin{aligned}
& E_{y}(x, y, B)=[4 \cdot Q(x, y, W, L) / \pi] \cdot E_{\text {Hall }} \quad \text { where } \quad E_{\text {Hall }}= \\
& j_{x} B / q n_{e}
\end{aligned}
$$

Here, $Q(x, y, W, L)$ is a quality factor that only depends on the samples width $W$ and length $L$ as well as the coordinates $x / y$ of the probe measurement. The latter can be obtained by the average change in potential as a function of $B$, which is unique for every point of the sample (See Supplementary Figure 3.32 and Figure $3.25 \mathrm{c}$ in the manuscript). $E_{y}(x, y, B)$ for the position in Figure $3.24 \mathrm{c}$,e in the manuscript is shown in Figure $3.25 \mathrm{e}$ in the manuscript. For this position the charge carrier concentration can be calculated by

$$
\begin{gathered}
n_{e}=[4 \cdot Q(x, y, W, L) / \pi] \cdot j_{\mathrm{x}}(B) \cdot B / E_{y}(x, y, B) / e \\
\approx(1.32 \pm 0.12) \cdot 10^{13} \mathrm{~cm}^{-2}
\end{gathered}
$$

where we took $j_{x}(B)$ again from the resistor network simulations. The error stems from the uncertainties of the fit to $E_{y}(x, y, B)$ in Figure $3.25 \mathrm{e}$ in the manuscript.

Besides the Hall measurements, the charge carrier concentration can additionally be determined by Scanning Tunneling Spectroscopy (STS) as shown in Supplementary Figure 3.33. By using thermovoltage imaging[201] in Supplementary Figure 3.33b,d we can distinguish between MLG and BLG. While BLG areas show standing wave patterns, MLG areas show a disordered electronic signature due to inhomogeneities in the buffer layer. In Supplementary Figure 3.33e we show STS measurements from different positions indicated in Supplementary Figure 3.33a. For the BLG areas we find the position of the Dirac point at $E_{D}=(-300 \pm 20) m V$, while for MLG it is located at $E_{D}=(-450 \pm 30) \mathrm{mV}$. This is in excellent agreement with ARPES data on these systems that extracted charge carrier concentration of $n_{\mathrm{MLG}}=1.0 \cdot 10^{13} \mathrm{~cm}^{-2}$ and $n_{\mathrm{BLG}}=1.3 \cdot 10^{13} \mathrm{~cm}^{-2}$ for these energetic positions of the Dirac point[137].

Since the surface area in Supplementary Figure 3.33a is mostly covered by BLG, this agrees well with the value obtained by the local Hall measurement.

\subsubsection{Supplementary Note 5}

Simulation of transport on the nano scale including local defects: For the simulation of the electric fields of the microscopic sample structure in Figure 3.25a and Figure $3.25 \mathrm{~b}$ in the manuscript we used the same resistor network method as described in Supplementary Note 1 [subsection 3.4.5.3]. The defects have been modeled by an isotropic conductivity tensor of $\sigma_{\mathrm{ML} / \mathrm{BL}}=0.05 \cdot \sigma_{0}$ and $\sigma_{\text {Wrinkle }}=0.3 \cdot \sigma_{0}$ and a transition width of $5 \mathrm{~nm}$. These values yield the same zero-field defect resistances as shown in Table 3.3 in the manuscript. To minimize the number of parameters we set $\sigma_{\mathrm{ML}}=\sigma_{\mathrm{BL}} \approx \sigma_{0}$, since both show similar values (See Table 3.3 
in the manuscript). For them the conductivity tensor in Supplementary Equation (3.36) was used.

The complete simulation of the microscopic sample structure can be found in Supplementary Figure 3.34. Here, we additionally included the current densities $j_{x} / j_{y}$ and the potential besides the electric field components $E_{x} / E_{y}$ shown in the manuscript.

Both experiment and simulations demonstrate how the influence of the magnetic field changes the current flow and enhances or depletes the electric field on the different facets of the large MLG hexagon. Consequently, the local voltage drop is in first approximation well-described by a semi-classical model. The resistance of our defects does not show an explicit dependence on magnetic field or the angle of incidence of the electrons as could be expected from quantum mechanical effects in graphene, e.g. Klein tunneling[86].

\subsubsection{Supplementary Note 6}

Evaluation of magnetotransport data: Here, we describe how to locally extract the sheet conductivity $\sigma$ and charge carrier concentration $n_{e}$ (part I) as well as the absolute defect resistance $\rho_{\text {Defect }}$ and its change with magnetic field (part II) as shown in Figure 3.27e-f in the manuscript. The measured local electric fields $E_{x}(B)$ (sheets) and voltage drops $\Delta V(B)$ (defects) are needed as experimental input. These are evaluated as averaged sections as shown in Figure 3.27d in the manuscript. Prior to that, the raw data has been evaluated as described in Ref. [201] to eliminate thermovoltage contributions.

\section{a) Conductivity and charge carrier concentration for MLG and BLG}

To extract the information about the MLG and BLG sheets we compared the experimentally observed electric fields $E_{x}(B)$ to finite element simulations.

For each dataset taken at a certain position of the sample simulations have been conducted changing both $\sigma$ and $n_{e}$ locally. This is necessary, since the change in field $E_{x}(B)$ is varying across the sample in the presence of a magnetic field (See Supplementary Figure 3.31c). The position of each dataset has been deduced from the change in the local potential $V(B)$ as demonstrated in Supplementary Note 3 [subsection 3.4.5.5].

Supplementary Figure 3.35 shows the simulated geometry. For the majority of the sample the macroscopic average values for conductivity $\langle\sigma\rangle$ and charge carrier concentration $\left\langle n_{\mathrm{e}}\right\rangle$ are used (See Table 3.3 in the manuscript and Supplementary Table 3.5). At the position where the experimental data was taken, both conductivity $\sigma$ and charge carrier concentration $n_{e}$ are varied in the simulation. A boundary 
region of lower conductivity was used to keep the current density constant across the sample. An area of $100 \mu \mathrm{m} \times 100 \mu \mathrm{m}$ was chosen which is reasonably large to ensure no boundary effects from the transition region and is still numerically feasible as well. This simulated geometry mimics the experimental situation that the voltage drop on the MLG and BLG sheets is different from the effective, macroscopic voltage drop which emerges from a mixture of defect scattering and sheet resistance.

Subsequently, the electric field $E_{x, \operatorname{sim}}(B)$ at this position has been simulated for different local conductivity $\sigma$ and charge carrier concentration $n_{e}$ in the area. Both quantities affect the conductivity tensor in Supplementary Equation (3.36) by changing $\frac{\sigma}{\left[1+(\mu B)^{2}\right]}$ as well as the off-diagonal terms. $E_{x, \operatorname{sim}}(B)$ is next compared to the experimental value $E_{x, \exp }(B)$ yielding the best fit of $\sigma$ and $n_{e}$.

The evaluation is demonstrated in Supplementary Figure 3.36a,b show the macroscopic potential across sample \#2 for $0 \mathrm{~T}$ and $5 \mathrm{~T}$, respectively. Additionally, the electric field in $x$-direction $E_{x}(B)$ is shown in Supplementary Figure 3.36c,d. Here, $\sigma$ and $n_{e}$ have been altered at the position of the dataset shown in Figure 3.27a-d in the manuscript. Both $n_{e}$ and $\sigma$ have been increased by $50 \%$ (arbitrary choice).

The consistency of the simulations can be checked as shown in Supplementary Figure $3.36 \mathrm{e}$, f by additionally calculating the sheet conductivity by

$$
1 / \sigma=E_{\|}(B) / j(B)
$$

where $j=\sqrt{j_{x}^{2}+j_{y}^{2}}$ is the total current density and $E_{\|}$is the electric field in direction of $j$. Thus, the sheet conductivity $\sigma$ can already be obtained from zero field measurements via the measured electric field $E_{x}(0 \mathrm{~T})$ and the current density $j_{x}(0 \mathrm{~T})=j(0 \mathrm{~T})$. Subsequently we use the magnetic field measurements to determine the charge carrier concentration $n_{e}$.

Supplementary Figure $3.36 \mathrm{~g}$, h show the change in $E_{x}(\sigma)$ for $0 \mathrm{~T}$ and $E_{x}\left(n_{e}\right)$ for 5 $\mathrm{T}$, respectively. The dots represent the experimentally measured values for the large MLG area in Figure 3.27a in the manuscript and allow to deduce $\sigma$ and $n_{e}$.

\section{b) Defect resistance as a function of $B$}

The defect resistance of all defects is calculated by

$$
\rho_{\text {Defect }}=\Delta V(B) / j_{x}(B)
$$

Here, the voltage drop $\Delta V(B)$ is obtained experimentally and the local current density $j_{x}(B)$ is taken from the simulations.

We are aware that in the analysis the inhomogeneities in current density will also be present on a local scale as suggested by 
Figure 3.26 in the manuscript and Supplementary Figure 3.34. This has to be taken into account for a quantitative analysis of local sheet resistances and defect line resistances.

Nevertheless, since the local current density yields on a larger scale $(500 \mathrm{~nm})$ the correct macroscopic value (Figure 3.25 in the manuscript), we approach the problem by averaging out the current density inhomogeneities by a sufficiently large number of data sets from different positions of the sample. In total we analyzed 32 datasets for MLG sheets, 47 for BLG sheets, 34 for ML/BL-interfaces, 29 for wrinkles and 3 for SiC-steps from 12 positions on 3 samples. Note that this includes datasets from the same sheet/defect, but different magnetic field $B$.

\subsubsection{Supplementary Note 7}

Discussion on local sheet conductivity and defect resistance: The results of the analysis discussed in the last section and already plotted in Figure 3.27e-f in the manuscript are shown again in Table 3.3 and Table 3.4 for the MLG and BLG sheets as well as for the defects.

For the MLG and BLG sheets the conductivity $\sigma$ is higher than macroscopically observed, which obviously stems from the fact that the macroscopic conductivity still contains the influence of local defects. The higher conductivity $\sigma$ of the bilayer compared to the monolayer can be explained with the higher charge carrier concentration $n_{e}$ as well as smaller influence of the underlying buffer layer inducing additional scattering. It is however not twice the monolayer value, since only one of the two bilayer bands is populated at the given doping level.[137] The large standard deviation $\Delta \sigma$ can be caused by changes in current density $j_{x}(B)$ that has to be taken from the FEM simulation and cannot be measured locally as discussed above. This would lead to incorrect values calculated by Supplementary Equation (3.40), since inhomogeneities, e.g. the presence of extended defects, can cause a locally higher or lower current density[42, 203]. While the statistics assures that these are averaged out, the variations also arise from the properties of the sample system as already discussed in the main text: local variations in mobility $\mu$ and charge carrier concentration $n_{e}$ lead to a large standard deviation $\Delta \sigma$ for the conductivity $\sigma$. This is supported by the additional large standard deviation $\Delta n_{e}$ found for $n_{e}$. Additionally, we found previously large variations in conductivity for evaluations by resistor network simulations taking account for the variations in the local current density[42]. Mobility variations can be caused by inhomogeneities in the graphene buffer layer as shown in Supplementary Figure 3.33b,d. This also affects $n_{e}$, since the buffer layer is influencing the doping level[157]. Additionally, stacking faults in bilayer graphene[69] can induce additional strain and differently stacked bilayer both influencing the mobility/conductivity. 
Despite the large $\Delta \sigma$ and $\Delta n_{e}$ caused by locally varying characteristics of the sample, the errors of the average value of $\sigma$ and $n_{e}$ remain small due to the large number of data points taken.

The role of the defects in the macroscopic measurements can be estimated by large-scale AFM measurements of all investigated samples as shown in Supplementary Figure 3.37. The defect density of steps and interfaces is decreasing with sample number. In contrast, the macroscopic average conductivity is increasing as can be seen from Supplementary Table 3.5.

Moreover, for short length scales the definition of a local conductivity is only welldefined, if the electron scattering length is small compared to the distance between steps and interfaces and for inhomogeneities in the local charge carrier concentration caused by the buffer layer. In other words, a classical conductivity is only welldefined in a diffusive regime when transport is not ballistic. Other transport studies investigated this transition in greater detail. Jobst et al. found a transport time of $\tau=\left(\hbar \sqrt{\pi n_{e}} / e v_{F}\right) \cdot \mu=0.045 \mathrm{ps}$ (derived from their Drude resistivity) in case of quasi-freestanding monolayer graphene on $\mathrm{SiC}$ [81]. Using the values from Table 3.3 and Table 3.4 in the manuscript $\left(n_{e} \approx 1 \cdot 10^{13} \mathrm{~cm}^{-2}, \mu \approx 0.2 \mathrm{~m}^{2} / \mathrm{V} \cdot \mathrm{s}\right)$ and $v_{F} \approx$ $1 \cdot 10^{6} \mathrm{~m} / \mathrm{s}$ yields $\tau \approx 0.07 \mathrm{ps}$ in good agreement with the above stated value. This can also be converted into units of length by $L=\sqrt{D \cdot \tau}$ with the diffusion constant $D=\left(n / e \cdot N_{s}\right) \cdot \mu$ and $N_{s}$ being the density of states[31]. For monolayer graphene with $N_{S}(E) \approx\left(2 / \pi\left[\hbar v_{F}\right]^{2}\right) \cdot|E|$ this yields $L \approx 50 \mathrm{~nm}$. Within weak-localization analysis even lower values have been found for the intravalley scattering time ranging between $10-20 \mathrm{~nm}[115,201]$. This is smaller than the typical distance between steps and interfaces $(>100 \mathrm{~nm})$. Consequently, evaluating the local conductivity on length scales of $\approx 100 \mathrm{~nm}$ is still a well-described quantity. This can for instance be seen in the voltage drop in Figure 3.27d. Within a MLG/BLG sheet the slope stays constant and does not vary spatially, except at the interfaces and on different sheets at different positions of the sample. For the latter, different local structures of the buffer layer can still lead to different conductivities (averaged on a scale of $\approx 100 \mathrm{~nm}$ ). This is shown in Figure 3.27e in the manuscript.

The absolute resistance values for localized scatterers (Table 3.4 in the manuscript) are in good agreement with Ji et al.[80]. While they did not investigate BLG wrinkles, we find that the values of this defect are larger than SiC-steps, but smaller than the ML/BL-interface. We propose that the transport mechanism here can be described by the transmission through a potential barrier induced by a difference in doping as discussed for the SiC-steps[108]. Since the width of the wrinkle ( 20 $\mathrm{nm}$ ) is larger than for the SiC-step ( $<2 \mathrm{~nm})$, the higher resistance for a BLG wrinkle is plausible. Since no electronic transition is involved, the magnitude is much smaller than for ML/BL-interfaces. 
Additionally, since the data of $\mathrm{Ji}$ et al. is taken at $77 \mathrm{~K} / 300 \mathrm{~K}$ the defect resistances seem to stay constant across a large temperature range for $\mathrm{ML} / \mathrm{BL}$-interfaces and $\mathrm{SiC}$-steps. Consequently, low-temperature effects such as quantum interference[81, 201] cannot play a significant role, which have been observed for some grain boundaries in graphene on $\mathrm{SiO}_{2}[210]$.

In contrast, for a potential barrier model for SiC-steps and BLG wrinkles (Supplementary Note 8 [subsection 3.4.5.10]) the transmission would not show a strict temperature-dependence. Accordingly, this is supported by the independence of the defect resistance with the magnetic field $B$ (Table 3.4 in the manuscript) in contrast to the sheets. Though the conductivity for the sheets given in Table 3.3 in the manuscript is not intrinsically depending on the magnetic field, the magnetic field leads to an increased time an electron needs to spend in the sheets thus increasing the resistance. This is not or insignificantly the case for the localized defects causing their contribution to vanish with magnetic field as seen in Figure $3.25 \mathrm{a}$ and Figure $3.27 \mathrm{~d}$ in the manuscript.

\subsubsection{Supplementary Note 8}

Transmission through a potential barrier with transverse magnetic field: To investigate the magnetotransport through a potential barrier as e.g. the SiC-steps and the bilayer wrinkles in the presence of a magnetic field we here treat the problem by wave function matching and by evaluating the transmission $\mathrm{T}$ through the barrier. Previously, it was argued that the detachment of the graphene sheet from the substrate is inducing a drastic change in doping, since $\mathrm{SiC}(0001)$-graphene is heavily $\mathrm{n}$-doped by the underlying buffer layer[80]. However, if it was simply a doping induced process, we would expect a quadratic increase, since the undoped graphene sheet would show the same magnetic field behavior as an n-doped one. However, the change in doping is inducing a potential barrier $V$ which is how we describe the defects here. The geometry of the model is depicted in Supplementary Figure 3.38.

For the conventional problem of transport through a potential barrier of the form

$$
\Phi(x)=\left\{\begin{array}{l}
V \text { if } x \in\left[-\frac{L}{2} ;+\frac{L}{2}\right] \\
0 \text { if } x \notin\left[-\frac{L}{2} ;+\frac{L}{2}\right]
\end{array}\right.
$$

the transmission can be written as 


$$
T=\frac{4 k_{x} k_{x}^{\prime} a}{a\left(k_{x}+k_{x}^{\prime}\right)^{2}+\sinh ^{2}(\sqrt{a} L)\left(a+k_{x}^{2}\right)\left(a+k_{x}^{\prime 2}\right)}
$$

With $a=\frac{2 m(V-E)}{\hbar^{2}}+k_{y}^{2}+k_{z}^{2}$. Under the influence of an external magnetic field the Hamiltonian has the form

$$
H=\frac{1}{2 m}(\mathbf{p}-q \mathbf{A})^{2}+q \Phi(x)
$$

using

$$
\mathbf{A}_{\mathrm{I}}=\left(0,-\frac{B L}{2}, 0\right)^{\mathrm{T}} ; \mathbf{A}_{\mathrm{II}}=(0, B x, 0)^{\mathrm{T}} ; \mathbf{A}_{\mathrm{III}}=\left(0,+\frac{B L}{2}, 0\right)^{\mathrm{T}}
$$

Here, the numbers indicate the different regions as indicated in Supplementary Figure 3.38a. We only include an explicit magnetic field dependence via the change in $x$ inside the barrier (region 2). To assure continuity of the wave functions the k-vectors before and after the barrier have to change

$$
\mathbf{k}_{\mathrm{F}}=\left(k_{x}, k_{y}\right) \rightarrow \mathbf{k}_{\mathrm{F}}^{\prime}=\left(\sqrt{k_{x}^{2}+\frac{2 e B L}{\hbar} k_{y}-\left(\frac{2 e B L}{\hbar}\right)^{2}}, k_{y}-\frac{e B L}{\hbar}\right)
$$

This leads to the modified transmission probability

$$
T=\frac{4 k_{x} \sqrt{k_{x}^{2}+\frac{2 e B L}{\hbar} k_{y}-\left(\frac{2 e B L}{\hbar}\right)^{2}} a}{a\left[k_{x}+\sqrt{k_{x}^{2}+\frac{2 e B L}{\hbar} k_{y}-\left(\frac{2 e B L}{\hbar}\right)^{2}}\right]^{2}+\sinh ^{2}(\sqrt{a} L)\left(a+k_{x}^{2}\right)\left(a+k_{x}^{2}+\frac{2 e B L}{\hbar} k_{y}-\left(\frac{2 e B L}{\hbar}\right)^{2}\right)}
$$

In front of the barrier the magnetic field leads to a different angle of incidence as shown in

Figure 3.26a and

Figure 3.26b in the manuscript. This change is relatively small and the angle can be estimated for $6 \mathrm{~T}$ by $\theta=\tan ^{-1}\left(E_{y} / E_{x}\right) \approx \tan ^{-1}(0.2)=11.3^{\circ}$ where the ratio $E_{y} / E_{x}$ has been estimated from Figure $3.25 \mathrm{c}$ and Figure $3.25 \mathrm{~d}$. We included it by a simple linear approximation $\theta=\left(11.3^{\circ}\right) /(6 \mathrm{~T}) \cdot B$ to determine the initial components $k_{x}=k_{\mathrm{F}} \cdot \cos \theta$ and $k_{y}=k_{\mathrm{F}} \cdot \sin \theta$. The length of the potential was chosen to be $L=2 \mathrm{~nm}$ and $L=20 \mathrm{~nm}$ for a SiC-step and a bilayer wrinkle, respectively. We additionally use $a=\frac{(V-E)}{\hbar v_{\mathrm{F}}}$ for graphene with $v_{\mathrm{F}}=1 \cdot 10^{6} \mathrm{~m} / \mathrm{s}$ [129]. Moreover, we choose the Fermi energies $E_{\mathrm{ML}}=0.44 \mathrm{eV}$ and $E_{\mathrm{BL}}=0.3 \mathrm{eV}$ (See Supplementary Note 5 [subsection 3.4.5.7]) as well as $E-V=80 \mathrm{meV}$ to adjust the electron concentration in the barrier to an intrinsic, 'undoped' level[86]. Since the transmission can be directly connected to the resistance in the Landauer Büttiker formalism[31] by

$$
\frac{1}{R}=G=\frac{2 \mathrm{e}^{2}}{\mathrm{~h}} M T
$$

We define the magnetoresistance as 


$$
\operatorname{MR}(B)=\frac{T^{-1}(B)}{T^{-1}(0)}-1
$$

The results for a SiC-step as well as a BLG wrinkle can be found in Supplementary Figure 3.38c. As can be seen the MR is small and also negative. For SiC steps we find $\operatorname{MR}(6 \mathrm{~T}) \approx-1 \%$ and for $\mathrm{BLG}$ wrinkles $\operatorname{MR}(6 \mathrm{~T}) \approx-4 \%$. These rather small changes fit to the experimentally found independence on magnetic field compared to the sheet resistance as described in the manuscript.

Thus, the defect resistance does not change much, if the scattering mechanism is induced by quantum tunneling through a potential barrier. Independent on the absolute value of the transmission, it barely changes due to a deflection inside the barrier and for different incident angles. By using Supplementary Equation (3.49) we followed for the sake of simplicity the Landauer approach including the contribution of the contacts of a ballistic conductor that contains the defect [31]. This has been previously used to describe the transmission of defects in graphene and $2 \mathrm{D}$ systems [30,122]. However, excluding the contacts would lead to $G \propto T /(1-T)$, which still yields a small variation with magnetic field. 


\section{Substitutional doping in epitaxial graphene using low energy ion implantation}

\subsection{Introduction}

Chapter 3 dealt with the influence of 1D structural defects (ML/BL-interfaces, wrinkles, steps) emerging from the growth process on local electron transport. In contrast, this chapter focuses on how 0D defects affect the local structural, electronic and transport properties. In particular substitutional dopant atoms and lattice defects are studied. In previous experiments, we found that they show no influence in STP experiments within the range of our current resolution [96]. Therefore, we study them by macroscopic transport measurements instead. Additionally, it is of interest how substitutional dopants are able to alter the electron concentration of graphene which is important for possible device fabrication.

While several benefits of foreign atoms in graphene have been discussed in the past, e.g. an enhanced sensitivity for sensors [113, 147], a straight-forward motivation is to tune the number of charge carriers in the sheet. In general, different strategies exist to change the charge carrier concentration in graphene, including adsorption of molecular adlayers $[110,161]$ and intercalation of other atomic species [146] between graphene and the substrate. The direct substitution with foreign atoms and a subsequent STM analysis has first been achieved by Zhao et al. for both nitrogen [216] and boron atoms [217] for graphene on copper foil, both shown in Figure 4.1. To do so they introduced different molecules into the reaction process for CVD-grown graphene (See subsection 2.1.2). Thus, they were able to demonstrate $\mathrm{p}$ - and $\mathrm{n}$-doping of graphene with boron and nitrogen, respectively. For epitaxial graphene on $\mathrm{SiC}$, implantation of nitrogen atoms has been achieved by plasma sources fed with $\mathrm{N}_{2}[83,154]$, thermal reactions with ammonia [193] as well as $\mathrm{N}_{2}$ ion irradiation [88, 176].

In this chapter we discuss our results for doping in graphene obtained with the method of low energy ion implantation introduced in subsection 2.1.3. As a postgrowth doping process it has the advantage of being independent of the graphene substrate. Additionally, it is highly flexible concerning the atomic doping species enabling us to analyze the effects of different kinds of foreign atoms; nitrogen, boron and carbon atoms in particular. Especially, the implantation of boron required for p-doping has rarely been studied and could only be achieved during the growth process so far [54, 175, 217]. While ion implantation is a widely-used technique in semiconductor device fabrication, implantation into a $2 \mathrm{D}$ sheet is non-trivial, since 
the kinetic energy of the electrons has to be low enough to neither reach further into the substrate nor create damage in the $2 \mathrm{D}$ sheet. One way to circumvent this problem is to implant at higher kinetic energies ( $>100 \mathrm{eV}$, which is usually easy to realize) followed by an additional annealing step to heal crystal lattice defects [88, 176]. Alternatively, our approach - low energy ion implantation used in the group of Prof. Hans Hofsäss - aims to decrease the kinetic energy enough that only implantation into the topmost layer is possible without creating further damage (or at least to minimize it). By combining classical molecular dynamics simulation with density functional theory, Åhlgren et al. found an optimum energy of around $50 \mathrm{eV}$ with increasing probability for adatom formation (vacancy creation) at lower (higher) energies, respectively [2].
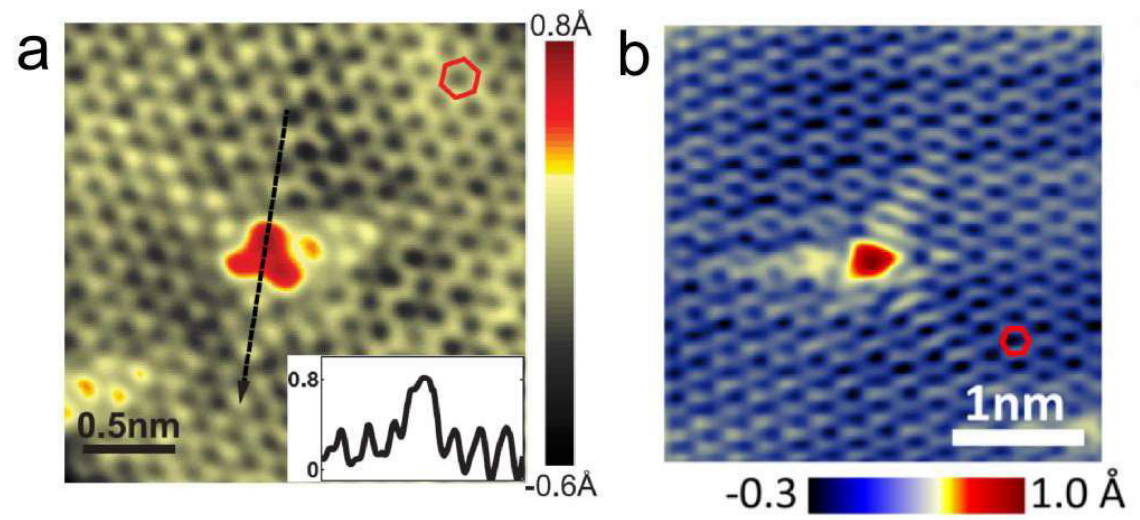

Figure 4.1: STM imaging of dopant atoms. (a) STM image of substitutional nitrogen atoms in $\mathrm{N}$-doped graphene on copper foil $\left(\mathrm{V}_{\text {bias }}=0.8 \mathrm{~V} / \mathrm{I}_{\text {set }}=0.8 \mathrm{nA}\right)$. [Reprinted (adapted) with permission from [216]. Copyright (2012) American Association for the Advancement of Science] (b) STM image of a single substitutional boron atom in B-doped graphene on copper foil $\left(\mathrm{V}_{\text {bias }}=-0.5 \mathrm{~V} / \mathrm{I}_{\text {set }}=0.5 \mathrm{nA}\right)$. Reprinted (adapted) with permission from Ref. [217]. Copyright (2013) American Chemical Society.

In section 4.2 we discuss the structural properties of implanted nitrogen atoms. Choosing a very low energy of $25 \mathrm{eV}$ in the implantation process, we aim to minimize the defect creation. By comparing the measured fluence with the density of nitrogen atoms observed in STM-topographies we find that during the doping process only 1 out of 10 atoms is implanted. Since between graphene sample preparation (Wenderoth group) and ion beam implantation (Hofsäss group) the samples are exposed to air and are not annealed again in the ion beam chamber, we explain this discrepancy by implantation into adsorbate adlayers in addition to ion adsorption and deflection of ions. Moreover, we find evidence that the adlayers are ordered which is triggered by the $6 \times 6$ corrugation with the underlying substrate and that they suppress implantation at specific sides. The implanted fraction is in agreement with samples prepared with the same method studied with high-resolution transmission electron microscopy [8]. Most importantly, we do not observe additional lattice defects after implantation which is certainly an advantage of this lowenergy approach. 
While the desired $n$-doping of nitrogen atoms has been observed in numerous cases, we show for the first time p-doping with boron atoms on SiC-graphene in our second study in section 4.3, which was at that point only demonstrated for CVD-graphene [217] or graphene on $\mathrm{Ni}(111)[54]^{18}$. In addition, we are able to resolve the boron atoms' electronic structure by STS for the first time.
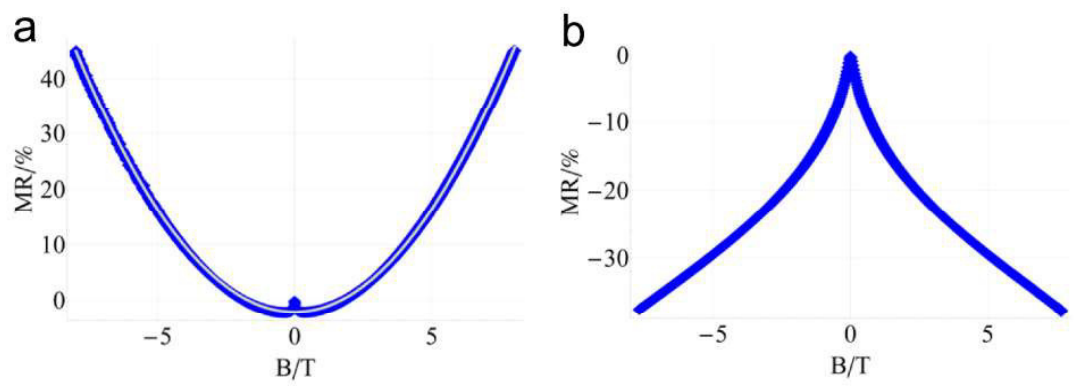

Figure 4.2: Magnetoresistance of nitrogen-implanted CVD-graphene on $\mathrm{SiO}_{2}$. (a) Pristine graphene showing a positive classical Lorentz magnetoresistance (MR), (b) N-doped graphene showing a strong negative MR due to weak localization. Both curves are taken at low temperatures (2.3 K). Reprinted (adapted) with permission from Ref. [152]. Copyright (2015) American Chemical Society.

Crucial for application is additionally a low influence of implantation on sample resistance and mobility by the additional atomic scale scattering centers. This was previously studied by Rein et al. who performed transport measurements in CVDgrown samples. They observed a large enhancement of weak localization (see subsection 1.2.5) after implantation leading to a strong negative magnetoresistance for nitrogen-implanted graphene by a CVD-method [152]. Both measurements on pristine and doped samples are shown in Figure 4.2. However, they did not perform any spatially resolving measurements and thus were not able to identify the predominant scattering centers.

The additional magnetotransport experiments ${ }^{19}$ also performed in section 4.3 demonstrate how doping atoms and atomic scale defects alter the transport properties of graphene. The significant advantage of our study is, that we precisely know the atomic-scale configuration derived from STM. Thus we can show that doping atoms alone only weakly contribute to the negative MR, but the main influence stems from additional atomic defects, e.g. vacancies.

Thus, studying the structural, electronic and transport properties in the same system allows us to extract a complete picture of the influence of substitutional atoms and lattice defects in graphene.

\footnotetext{
18 Another implantation for SiC-bilayer Graphene studied by STM was published shortly after our work by Telychko et al. [175].

19 These experiments have been conducted using the PPMS introduced in subsection 2.2.5. We would like to thank the I. Physikalisches Institut for using their system to conduct these transport measurements
} 


\subsection{Short-range ordering of ion-implanted nitrogen at- oms in SiC-graphene}

P. Willke, J. A. Amani, S. Thakur, S. Weikert, T. Druga, K. Maiti, H. Hofsäss and M. Wenderoth

Applied Physics Letters 105, 111605 (2014)

DOI: $10.1063 / 1.4895801$

We perform a structural analysis of nitrogen-doped graphene on $\mathrm{SiC}(0001)$ prepared by ultra low-energy ion bombardment. Using scanning tunneling microscopy, we show that nitrogen atoms are incorporated almost exclusively as graphitic substitution in the graphene honeycomb lattice. With an irradiation energy of $25 \mathrm{eV}$ and a fluence of approximately $5 \times 10^{14} \mathrm{~cm}^{-2}$, we achieve a nitrogen content of around $1 \%$. By quantitatively comparing the position of the $\mathrm{N}$-atoms in the topography measurements with simulated random distributions, we find statistically significant short-range correlations. Consequently, we are able to show that the dopants arrange preferably at lattice sites given by the $6 \times 6$-reconstruction of the underlying substrate. This selective incorporation is most likely triggered by adsorbate layers present during the ion bombardment. This study identifies low-energy ion irradiation as a promising method for controlled doping in epitaxial graphene.

Graphene, a single layer of $\mathrm{sp}^{2}$-bonded carbon atoms, is a potential material for electronic devices and due to its compatibility with existing fabrication processes epitaxial grown graphene on silicon carbide $(\mathrm{SiC})[13,46]$ is an excellent system for future application. To tailor the electronic properties down to the nanometer scale, doping of the graphene sheet is a necessary method. While doping can be achieved by different forms of adsorption.[26, 161]. direct incorporation of doping atoms into the graphene honeycomb lattice has the advantage of enhanced stability. In graphene samples grown by chemical vapor deposition (CVD), doping has been achieved by introducing additional components during the growth process and was studied up to now in great detail.[184, 193, 212, 216, 217]

For epitaxial-grown graphene on $\mathrm{SiC}$, it was shown that n-type doping can be realized by thermal reactions with molecules.[36, 193] Recently, nitrogen doping of $\mathrm{SiC}$-graphene has been achieved by different forms of atomic nitrogen flux[83, 88, $154,189]$ leading to an effective doping.[83] Thus, incorporated nitrogen is observed in different kinds of atomic configurations, including graphitic-, pyridine-, and pyrrolic-like nitrogen atoms.[88] Moreover, the creation of nitrogen-vacancycomplexes[154] and different types of atomic lattice defects[83] have been identified. Nevertheless, a controlled ion substitution conserving the graphene lattice is 
still a challenging task: Recent molecular dynamics simulations of low energy nitrogen and boron ion irradiation of graphene predict high substitution fractions exceeding $50 \%$ for ion energies around $50 \mathrm{eV}$ [2]. In a substitution process, one $\mathrm{C}$ atom is replaced by a N or B atom. For even lower ion energies, the substitution fraction decreases, but for energies below approximately $40 \mathrm{eV}$, the generation of single vacancy defects is strongly suppressed. Therefore, ultra low energy ion implantation appears very attractive for substitutional doping of graphene without significant generation of point defects. We have recently demonstrated efficient doping of graphene by ${ }^{14} \mathrm{~N}^{+}$and ${ }^{11} \mathrm{~B}^{+}$ion irradiation using mass-selected ions with ion energies of $25-35 \mathrm{eV}$ for graphene on other substrates.[8, 206] High resolution transmission electron microscope (HR-TEM) analyses demonstrated substitutional doping with $\mathrm{N}$ and $\mathrm{B}$ atoms in a controlled manner. We here present the first STM (scanning tunneling microscopy) study of nitrogen incorporation in epitaxial graphene on $\mathrm{SiC}(0001)$ (Si-terminated) by low-energy mass-selected ${ }^{14} \mathrm{~N}^{+}$ion implantation. By using scanning tunneling microscopy, we can study the incorporated atoms in a direct way and gain insight in their spatial distribution. We find that the incorporation of $\mathrm{N}$-atoms is influenced by the $\mathrm{SiC}$-substrate: performing a statistical evaluation of the dopant distribution, we find that the $\mathrm{N}$-atoms are preferentially found on the topographically high regions of the $6 \times 6$ graphene-SiC quasi-reconstruction.

The preparation of our graphene samples was done by thermal desorption.[155] By heating a nitrogen-doped $6 \mathrm{H}-\mathrm{SiC}(0001)$-wafer for $2 \mathrm{~min}$ at $1400^{\circ} \mathrm{C}$ under ultra high vacuum (UHV) conditions, we obtained samples covered by monolayer graphene. In a second step, the prepared graphene samples were ex-situ transferred and reinserted into our mass-selective ion beam deposition system (base pressure $<5 \times 10^{-8} \mathrm{mbar}$ ). This necessary transfer step is crucial for the further implantation process, since under environmental conditions, adsorbate layers can form on the topmost graphene layer. For the implantation, a $30 \mathrm{keV}$ ion beam is decelerated to energies down to about a maximum value of $25 \mathrm{eV}$ leading to an almost uniform irradiation of an area of about $2 \mathrm{~cm}^{2}$. The implantation with mass-selected ${ }^{14} \mathrm{~N}^{+}-$ ions were performed with a fluence of approximately $5 \times 10^{14} \mathrm{~cm}^{-2}$. If all ions were incorporated by substitution into the uppermost graphene layer, this would result in an $\mathrm{N}$-content of around $13 \%$. Since we can precisely measure the deposited ion charge, it is easily possible to control the total irradiation fluence (ions per unit area) through the irradiation time. However, because of the tail of low energy ions and a reduced substitution probability below $50 \mathrm{eV}$ as predicted by molecular dynamics simulations,[2] the substitution fraction will be lower. Our own Monte Carlo simulations using the SDTrim software package lead to an incorporation rate of about $80 \%$ of the incorporated ions found in the top graphene layer (See section 4.2.2). About $20 \%$ are deposited into the underneath buffer layer. Additionally, 
sputter effects are negligible at these low ion energies leading to no defect generation. A process that clearly suppresses the incorporation rate is the effect of the ambient adsorbate film obtained ex-situ. In this case, a significant amount gets incorporated into this film. By adding a water film onto the graphene sheet in the simulation, we find that an adlayer film of $1.25 \mathrm{~nm}$ is sufficient to decrease the amount of implanted nitrogen to $0 \%$.

(a)

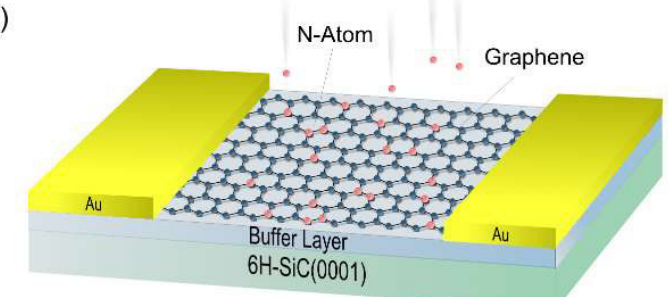

(b)

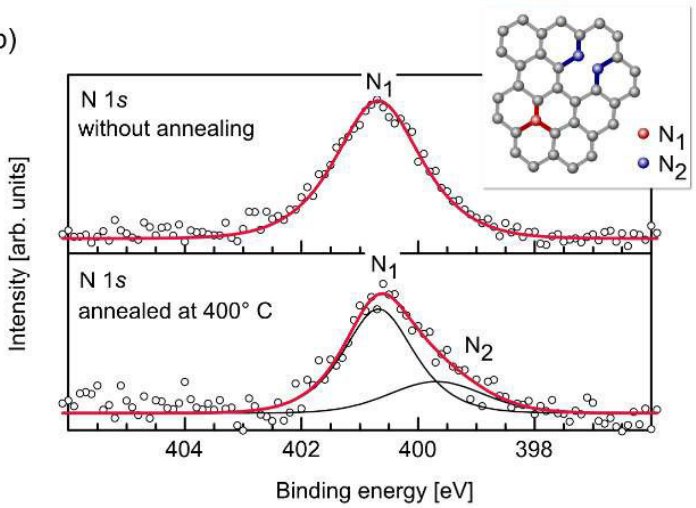

Figure 4.3: (a) Schematic of the experimental setup: epitaxial-grown graphene prepared by thermal sublimation on $6 \mathrm{H}-\mathrm{SiC}(0001)$ is irradiated by ${ }^{14} \mathrm{~N}^{+}$-ions with a kinetic energy of $25 \mathrm{eV}$. For STM-measurements, gold contacts have been added. (b) $\mathrm{N} 1 \mathrm{~s}$ spectra of graphene after nitrogen implanted without additional heating (red) and heating to $400{ }^{\circ} \mathrm{C}$.

The nitrogen irradiated graphene samples as depicted in Figure 4.3a were heated at $400^{\circ} \mathrm{C}$ in UHV before the STM measurements to thermally desorb adsorbate layers present on the sample. To assure that this step is not changing the atomic structure of the incorporated nitrogen atoms, high resolution x-ray photoemission spectroscopic (XPS) measurements were carried out using a Gammadata Scienta analyzer and monochromatic $\mathrm{x}$-ray source. The experimental spectra collected at room temperature before and after thermal desorption of the sample are shown in Figure $4.3 \mathrm{~b}$. For the latter, the sample was heated to $400^{\circ} \mathrm{C}$ by a resistive heating setup in a vacuum better than $1 \times 10^{-10}$ Torr. For the unannealed case, the $\mathrm{N} 1 \mathrm{~s}$ spectra exhibit a dominant peak $\left(\mathrm{N}_{1}\right)$ at about $400.8 \mathrm{eV}$ binding energy corresponding to a substitutional (graphitic) incorporation of the nitrogen atoms.[88, 184] After annealing, the sample shows a line shape asymmetry due to multiple species as observed in other systems with our setup. $[143,165]$ This can be fitted with an additional peak at lower binding energy around $399 \mathrm{eV}\left(\mathrm{N}_{2}\right)$ representing the pyridinic form. $[88,184]$ We attribute the appearance of this pyridinic nitrogen after heating 
to the fact that such signals were shifted by the adsorbed oxygen in the unheated sample: Since nitrogen will gain positive valency when it makes a bond with oxygen, the binding energy of the $\mathrm{N} 1 \mathrm{~s}$ signal will increase. Such energy shifts have been observed for the present system[184] ${ }^{20}$ as well as other systems.[143] These results show that the vast majority of ions were incorporated in the graphitic form.

Next, we used a homebuilt STM setup operating at $77 \mathrm{~K}$ and a base pressure $<1 \times 10^{-11}$ mbar to analyze the spatial distribution of the $\mathrm{N}$-dopants in the graphene lattice. Figure 4.4a shows an example of a pristine graphene sample without nitrogen bombardment. Here, two different lattice periodicities are visible: first, the atomic honeycomb lattice of the hexagonal graphene layer, which is also enlarged in the inset. Second, the larger $6 \times 6$-corrugation induced by the underlying interface layer. This modulation becomes visible in scanning probe experiments and consists of both electronic contrast[187] as well as actual height corrugation.[51] In Figure 4.4b, we now show a typical topography image after the ion implantation: Still, the graphene lattice corrugation and the $6 \times 6$-modulation are visible, but the contrast is dominated by the bright circular shaped features of the nitrogen dopants. This electronic contrast of substitutional $\mathrm{N}$-atoms has been already observed in other STM-experiments[83, 216] as well as predicted by theory.[218] In the inset, we show a high resolution image showing a single dopant atom, the graphene honeycomb lattice and the $6 \times 6$-modulation. Their scattering pattern reveals a threefold symmetry that occurs in two mirror-symmetric orientations. This has been attributed to the different orientations of sublattices.[83, 216] The total number of dopants in the image is $\mathrm{N}_{\text {tot }}=135$ with 56 on the one and 59 on the other sublattice. For 20 dopants, it was not possible to determine the orientation due to overlaps in the electronic contrast. In accordance with topography measurements at other positions, we could find that both sublattice positions are equally favored by the $\mathrm{N}$-atoms in contrast to recent studies on CVD-grown graphene.[212, 217] For the given image, this yields a dopant density of $\rho_{N}=0.39 \mathrm{~nm}^{-2}=3.9 \times 10^{13} \mathrm{~cm}^{-2}$. On average, we obtained $\rho_{N}=(0.35 \pm 0.10) \mathrm{nm}^{-2} .{ }^{21}$ For a C-atom density in graphene of $\rho_{C}=38.5 \mathrm{~nm}^{-2}$, we obtain a dopant concentration that is slightly below $1 \%$. Though this is in good agreement with our recent experiments on freestanding graphene,[8] it is still a factor of 10 lower than the predicted number, which we obtain from the measured total charge transfer and the simulations. According to the simulation, this cannot be explained by deflection and incorporation into the

\footnotetext{
${ }^{20}$ An energy shift due to gold is seen by Ref. [184] although in the other direction as gold is less electronegative.

${ }^{21}$ For a second sample prepared with a fluence of $3 \cdot 10^{14} \mathrm{~cm}^{-2}$, we obtained a dopant density of $(0.17 \pm 0.10) \mathrm{nm}^{-2}$. This demonstrates nicely the dose dependent implantation rate by ion implantation.
} 
substrate only, but an incorporation in the adsorbate film present during the irradiation on top of graphene is likely to decrease the effective doping concentration. Therefore, we assume that the adsorbed films hinder the incorporation process in certain regions, while in others, it stays unaffected depending on the exact geometry of the film. In addition to that, we cannot exclude that the incorporation leads to a local charging: Since in contrast to the graphene sheet, the adsorbate film is likely to be weakly conducting, ions which get incorporated into it cannot dissipate their charge. This leads to a charging-induced deflection and deceleration of the subsequently arriving ions from the adsorbate areas. Similar to the low energy tail of the $25 \mathrm{eV}$ beam distribution sufficiently decelerated ions will not get incorporated, which further decreases the observed number of nitrogen atoms. As a consequence of the importance of the adlayer, we analyze the spatial distribution of the nitrogen atoms in more detail.

As a first feature, we identify an almost perfect hexagonal doping structure (indicated in red) around a topographically low region of the $6 \times 6$ modulation in the center of Figure 4.4b. Such a complex structure is very unlikely to be formed accidently. ${ }^{22}$ Quite the contrary, the general observation from topographic measurements is that the $\mathrm{N}$-atoms avoid the holes of the reconstruction and arrange around them. Apparently, the locally different structural and electronic properties of the graphene $6 \times 6$-corrugation trigger the selective incorporation in the ion implantation process. For epitaxial graphene on different substrates, it has been shown that the self-assembly of single molecules and films can be influenced by the substrates' geometry.[109, 128, 158] In case of $\mathrm{SiC}(0001)$ ordering of $\mathrm{H}_{2}, \mathrm{Cs}$, and CIAIPc along of the $6 \times 6$-corrugation has been reported. $[6,75,166]$ This has been attributed to the local change in topographic and electronic structure. In our case, this could also affect the incorporation probability depending on the side of the impact. But changing the interlayer distance for the graphene sheet in the simulation to model the effect of the height corrugation did not show a change in incorporation probability due to the only small topographic change. Moreover, comparing molecular dynamics simulation of the implantation process for graphene and carbon nanotubes did not show a significant difference in the incorporation process as demonstrated by others.[2] Since both carbon-based systems have different electronic structures, we conclude that structural properties dominate the ion implantation process over electronic properties as well as the interlayer distance. Thermal diffusion in the heating process is a second possibility for the ordering. But we only observe little change in the XPS spectra so that no other nitrogen defects than the rather stable graphitic type defects could diffuse to the edges.

\footnotetext{
22 Using basic statistics, this can be more quantified: The number of $\mathrm{C}$ atoms in the $(6 \sqrt{3} \times 6 \sqrt{3}) R 30^{\circ}$ super cell is 338 (See Ref. [155]) and, consequently, in the $(6 \times 6)$-modulation approximately 113 . The probability of at least 4 correct spots on the edges of $(6 \times 6)$-modulation is given by $\left(\begin{array}{c}6 \\ 4\end{array}\right) /\left(\begin{array}{c}113 \\ 6\end{array}\right)=6 \cdot 10^{-7} \%$.
} 
Moreover, due to the lack of vacancies, the diffusion process requires the direct switching of a nitrogen atom with several carbon atoms. A third possibility is an ordering of the adsorbate layers: An arrangement of the surface molecules under ambient conditions similar to the adatom structures[109, 128, 158] could serve as a shadow mask that hinders the ions from implantation. For bilayer graphene exposed to ambient air, ordered adsorbate films have been recently found, while the film on monolayer graphene is rather sophisticated.[195]

To quantify this observation of short-range ordering, we perform a statistical analysis in the following. First, we evaluate the distance distribution of the dopants to the $6 \times 6$-modulation centers. Second, we compute the radial pair-distribution function for the dopants, which is the number of atoms that are in a certain shell around a central atom. Both evaluation methods were done for the measured data and were compared to simulated random distributions.

In Figure 4.5a and Figure 4.5b, we show a $16.5 \times 16.5 \mathrm{~nm}^{2}$ topography image in which due to different bias voltages $\cup_{\text {Bias, }}$, the $6 \times 6$-reconstruction and the dopants are pronounced, respectively. From that, we are able to extract the exact spatial positions of the nitrogen atoms as well as the overlattice structure, especially the positions of the holes. This is indicated in Figure 4.5c: triangles for the dopants (different colors and orientation mark the position on different sublattices) and dark dots for the central reconstruction positions. The position of the latter is still a little bit subtle: On the one hand, due to drift in the image, we do not obtain a perfect hexagonal lattice and on the other hand, the actual central position might be covered by the electronic contrast of a dopant atom. Therefore, we used 2 lattices
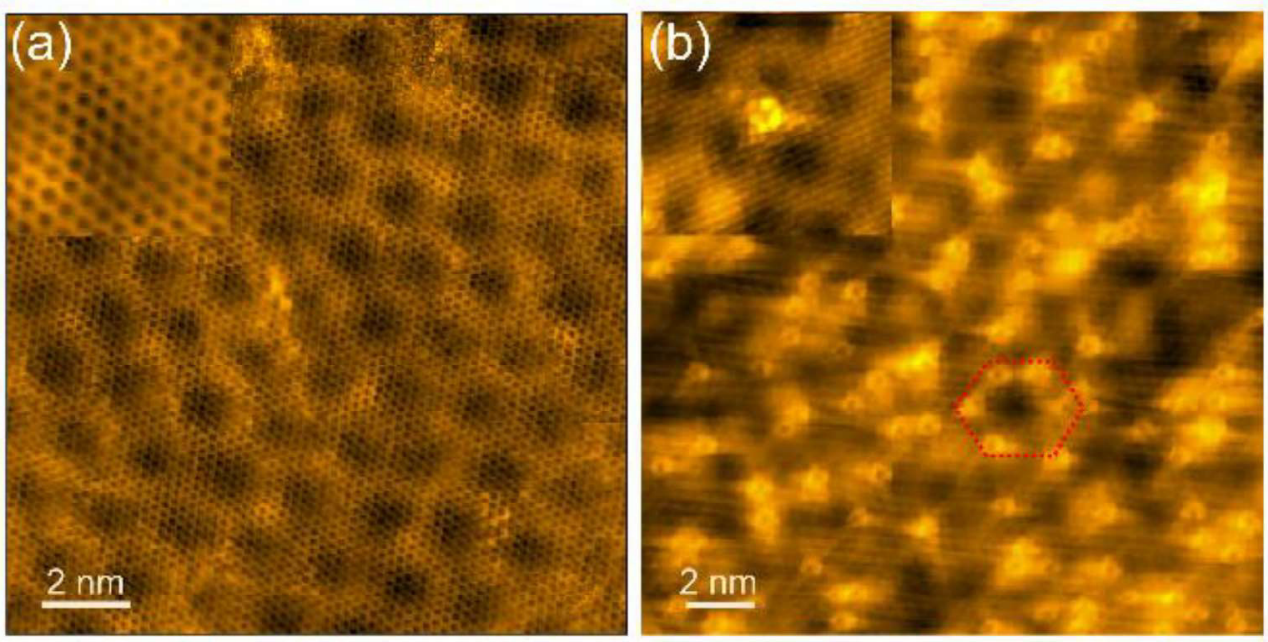

Figure 4.4: (a) $14.5 \times 14.5 \mathrm{~nm}^{2}$ topography of pristine monolayer graphene $\left(\mathrm{U}_{\text {Bias }}=-40 \mathrm{mV}, \mathrm{I}_{\mathrm{T}}=70 \mathrm{pA}\right)$ dominated by two corrugations: The graphene lattice (See $2.5 \times 2.5 \mathrm{~nm}^{2}$ inset) and the $6 \times 6$-modulation of the $\operatorname{SiC}(0001)$ surface. (b) $18.5 \times 18.5 \mathrm{~nm}^{2}$ topography of graphene after nitrogen irradiation $\left(U_{\text {Bias }}=100 \mathrm{mV}\right.$, $\left.I_{T}=50 \mathrm{pA}\right)$ revealing several nitrogen scattering patterns. Inset: high resolution $6 \times 6 \mathrm{~nm}^{2}$ image of a nitrogen dopant with again both lattice corrugations $\left(U_{\text {Bias }}=-50 \mathrm{mV}\right.$, $\left.I_{T}=50 \mathrm{pA}\right)$. The red line marks an almost perfect hexagonal doping structure. 
for the further analysis: one that has been obtained by the visible topographic minima (black dots) decreasing the influence of drift. The second one was obtained by averaging along each line of the black dots and using the intercepts of these black lines (also indicated) as averaged points. These points (marked in yellow) are minimizing the effect of covered reconstruction holes.

Our algorithm calculates for each dopant (triangle), the minimal distance to an overlattice minimum (dot). Figure $4.5 \mathrm{~d}$ shows the distance distribution for the measured data and the average over 1000 simulated random distributions of the dopant distributions with the given lattices. The box size for the histogram is $2 \AA$. As can be seen, for both lattice configurations, the random distribution yields a lower mean value and a higher standard deviation for the fitted Gaussian. We obtain, for the black (yellow) dot lattice, an increase of the standard deviation of $44 \%$ $(34 \%)$, the mean value is increased by $21 \%(14 \%)$.

Consequently, the dopants are significantly farer away from the reconstruction hole centers as it would be expected from a complete random distribution. This clearly demonstrates the tendency of the dopants not only to get incorporated in a nonrandom way, but also to prefer sites at the corners or topographically higher regions of the $6 \times 6$-modulation.

In the second part, we want to focus on the pair-distribution function $\mathrm{R}$ of the dopants. This quantity has already been used to confirm short-range correlations of dopants in semiconductor-crystals.[67] It is defined as

$$
R(r)=\frac{N_{\text {Data }}(r)}{N_{\text {Rand }}(r)}
$$

where $N_{\text {Data }}$ is the number of $\mathrm{N}$-atoms in a finite shell at a certain radius $r$ from a central $\mathrm{N}$-atom and $N_{\text {Rand }}$ is the equivalent number found for again simulated random distributions. Both numbers are evaluated for all atoms in Figure 4.5c. The distribution of $N_{\text {Data }}$ and $N_{\text {Rand }}$ is shown in Figure $4.5 \mathrm{e}$. We also distinguished between the correlation of $\mathrm{N}$-atoms on same/different sublattices that are shown in $\mathrm{red} / \mathrm{blue}$. The average distribution of 1000 simulated random maps is shown in black. Here, a sublattice-dependent evaluation does, of course, not show any difference, so that we only plotted a single function. For small values of $r$, all functions increase linearly as a function of radius because the total number is here proportional to the shell area at distance $r$. For larger distances, the finite size of the atom map leads first to a flattening followed by a decrease of the total numbers.

In Figure 4.5f, we now plot the pair-distribution, the normalization of the data in Figure 4.5e. For the hypothesis of a perfectly random distribution, it should yield a value of 1 independent of the distance. The $2 \sigma$-confidence interval resulting from the finite number of dopants is shown as black lines in the plot to pronounce significant deviations. For comparison, different box sizes of 2,3 , and $4 \AA$ are shown. 
The pair-distribution function reveals two main features that significantly deviate from random results. First, strong short-range correlations highlighted by the grey shading can be found for small $r$, especially for the pair-distribution function of different sublattice atoms (blue line). The peak value corresponds to a value between $4 \sigma(3 \AA)$ and $2.5 \sigma(4 \AA)$. Consequently, the assumption that the nitrogen atoms

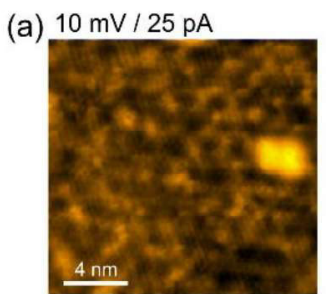

(c)

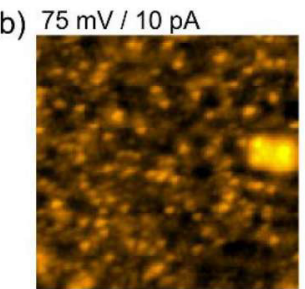

(d)

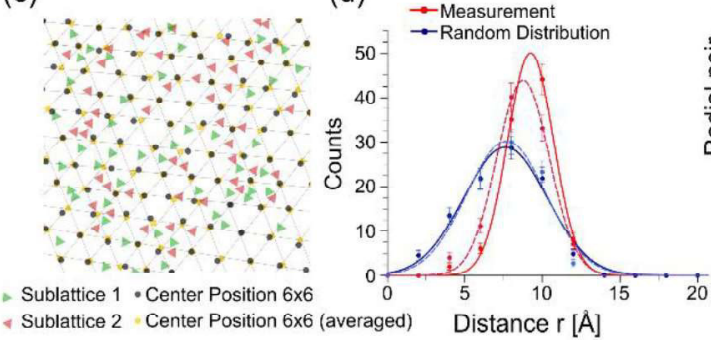

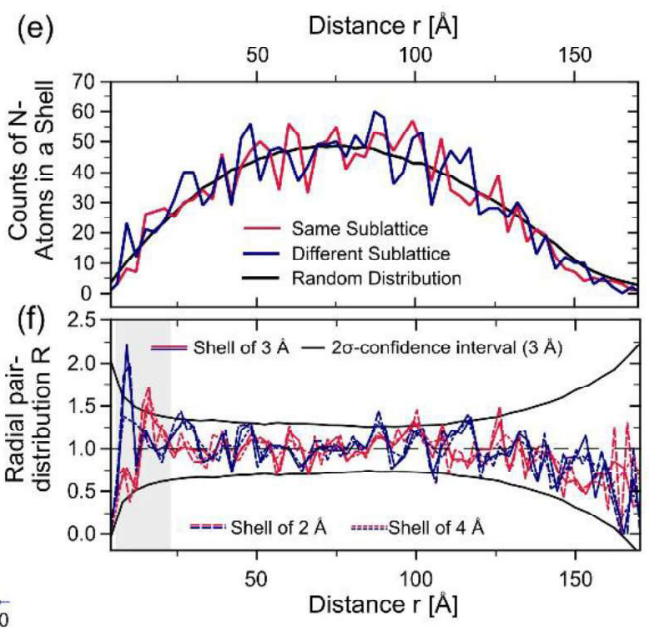

Figure 4.5: $16.5 \times 16.5 \mathrm{~nm}^{2}$ topography for (a) $U_{\text {Bias }}=10 \mathrm{mV}, I_{\mathrm{T}}=25 \mathrm{pA}$ and (b) $U_{\text {Bias }}=75 \mathrm{mV}, I_{T}=10 \mathrm{pA}$ pronouncing the $6 \times 6$ corrugation and the position of the dopants, respectively. (c) Map of the hole positions of the $6 \times 6$-modulation (black/yellow dots) and the dopant position and orientation (green and red triangles for different sublattices) extracted from the multibias images in (a) and (b). (d) Distribution of the average distance of a dopant from a $6 \times 6$-modulation center (black points) for the measured dopants (red) and for an average distribution of 1000 random configurations (blue). The dotted line shows the results for the yellow points that mark the intercepts of the black lines representing averaged $6 \times 6$-modulation centers. (e) Counts of $\mathrm{N}$ atoms in the measured (red, blue) and in the simulated random maps (black) within a $5 \AA$ shell as a function of radius from a central $\mathrm{N}$ atom. The red line describes $\mathrm{N}$-atoms that are in the same sublattice, the blue line those that are in a different sublattice than the central $\mathrm{N}$ atom. (f) Radial pair-distribution function $\mathrm{R}$ for the measured maps calculated from (e). Same sublattices are again shown in red, different ones in blue. The most significant peak is found at $r \approx 10 \AA$.

are arranged randomly on a short range scale can be rejected with a statistical significance of about $99 \%$. Moreover, also correlations for the same sublattice are found with a slightly smaller significance. The first peak positions for different (same) sublattice correlation of roughly $10 \AA$ (16 $\AA$ ) can be linked qualitatively with the ordered hexagon shown in Figure 4.4b: here, each pair of nearest $\mathrm{N}$-atoms has opposite sublattices and each pair of next-nearest $\mathrm{N}$-atoms has the same sublattice configuration. Since the lattice distance of the $6 \times 6$-modulation is $a_{6 \times 6}=6$ $\times a_{\mathrm{sic}}=6 \times 3.08 \AA=18.5 \AA$, the tendency for short-range ordering shows further evidence for the connection to the underlying reconstruction. The second feature is a decrease in correlation at distances above $150 \AA$. Such a decrease can be the result of clusters on this length scale. Indeed, a large dopant-free area is found at 
the lower right corner that is minimizing the statistics for high distances. On the contrary, larger topographies at other positions do not confirm this correlation and the deviations are also still close to the $2 \sigma$-confidence interval.

In summary, N-doping of graphene was attained by mass-selective low-energy ion implantation in epitaxial-grown samples. XPS, Monte Carlo simulations and STM images showed an almost perfect incorporation in the graphitic form. We were able to demonstrate that an ordered arrangement of the nitrogen atoms occurs triggered by the $6 \times 6$-modulation of the underlying SiC-crystal. We make adsorbate layers forming a structured mask responsible for this ordered implantation in agreement with the observed decreased incorporation compared to the measured fluence. This stays an open task for future implantation experiments, but also demonstrates the possibility of nanopatterned ion implantation by using self-assembled adsorbate films opening the way for ordered doping networks down to the atomic scale.

\subsubsection{Acknowledgement}

This work was supported by the Deutsche Forschungsgemeinschaft (DFG) priority program 1459 Graphene. Financial support by the DFG under Contract No. HO1125/21-1 was also acknowledged. The author, S.T. acknowledges financial support from India office, University of Göttingen, Germany, under the academic cooperation between TIFR, Mumbai and Georg August University, Göttingen.

\subsubsection{Supplementary Information}

\subsubsection{Details of Monte Carlo Simulations}

To simulate the incorporation process of the nitrogen atoms into the epitaxial graphene-SiC system as depicted in Figure 4.3a, we performed Monte Carlo simulations using the SDTrimSP software package.[45]

The graphene layer and the buffer layer were modeled by an amorphous carbon layer on top of a $\mathrm{SiC}$ crystal. The implantation of nitrogen atoms $\left({ }^{14} \mathrm{~N}^{+}\right)$was performed for an energy of $25 \mathrm{eV}$ as in the experiments. We simulated the geometries for a monolayer and a bilayer system with in total two and three amorphous carbon layers, respectively. The systems are depicted in Figure 4.6. Moreover, we show the distribution of different atoms in Table 4.1 and Table 4.2.

As stated in the manuscript, we observe no sputtering. A minor diffusion of silicon and carbon into the buffer layer and carbon atoms from the latter into the SiC substrate is observed. For monolayer graphene $80 \%$ of the nitrogen atoms are incorporated into the graphene layer and $20 \%$ into the buffer layer, which hardly changes for the bilayer system. 
(a) $25 \mathrm{eV}^{14} \mathrm{~N}^{+} \rightarrow \mathrm{C} / \mathrm{SiC}$

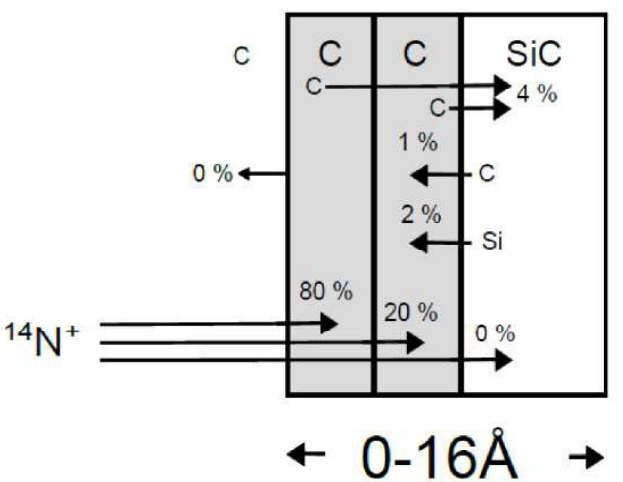

(b)

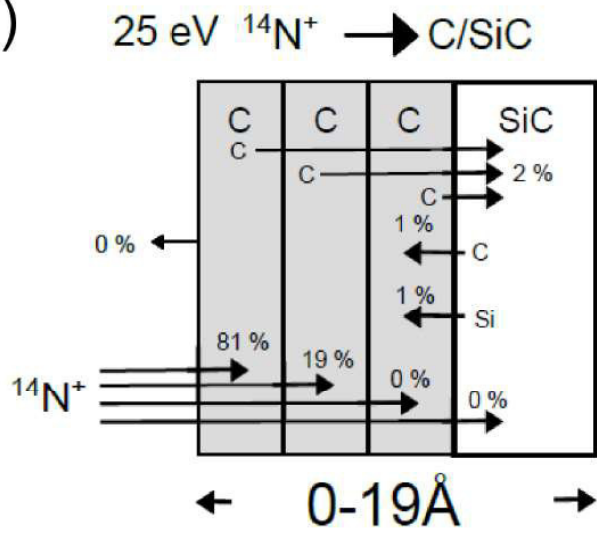

Figure 4.6: Layer dependent distribution of the nitrogen atoms for (a) monolayer graphene and (b) bilayer graphene.

\begin{tabular}{r|ccccc}
\hline Depth $[\AA]$ & $0-3$ & $3-6.4$ & $6.4-11.4$ & $11.4-16$ & Total \\
\hline Nitrogen & 80 & 20 & 0 & 0 & 100 \\
a-Carbon & 44 & 52 & 4 & 0 & 100 \\
Silicon & 0 & 2 & 50 & 48 & 100 \\
Carbon & 0 & 1 & 50 & 49 & 100
\end{tabular}

Table 4.1. Distribution (percentage) for different atoms in the layers of the monolayer graphene system.

\begin{tabular}{r|cccccc}
\hline Depth $[\AA]$ & $0-3$ & $3-6$ & $6-9.4$ & $9.4-14.4$ & $14.4-19$ & Total \\
\hline Nitrogen & 81 & 19 & 0 & 0 & 0 & 100 \\
a-Carbon & 29 & 32 & 37 & 2 & 0 & 100 \\
Silicon & 0 & 0 & 1 & 51 & 48 & 100 \\
Carbon & 0 & 0 & 1 & 51 & 48 & 100
\end{tabular}

Table 4.2. Distribution (percentage) for different atoms in the layers of the bilayer graphene system.

\subsubsection{Influence of Adlayers}

To estimate the influence of adsorbates for the incorporation probability into the graphene sheet we added an adlayer of water as a typical adsorbate in ambient conditions. The simulations have been done for different numbers of "layers" with the density of water at room temperature. The thickness of one layer has been chosen to be $2.5 \AA$, which is in the order of magnitude as a graphene layer. As can be seen in Figure 4.7d this decreases the incorporation linearly with increasing adlayer thickness. For a total thickness about $1.25 \mathrm{~nm}$ the incorporation drops to $0 \%$. Since this is in a reasonable order of magnitude for adlayers on graphene 
[195], the simulation supports the experimentally observed drop in incorporation fraction and local ordered implantation due to ordered adlayers.

(a)

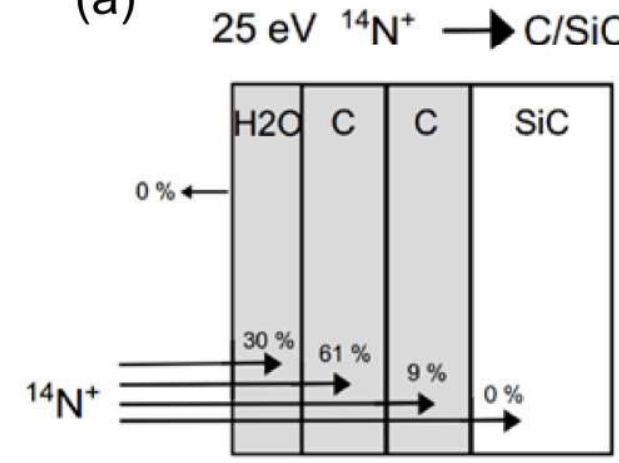

(b) $25 \mathrm{eV}^{14} \mathrm{~N}^{+} \rightarrow \mathrm{C} / \mathrm{SiC}$

(c) $25 \mathrm{eV}^{14} \mathrm{~N}^{+} \rightarrow \mathrm{C} / \mathrm{SiC}$
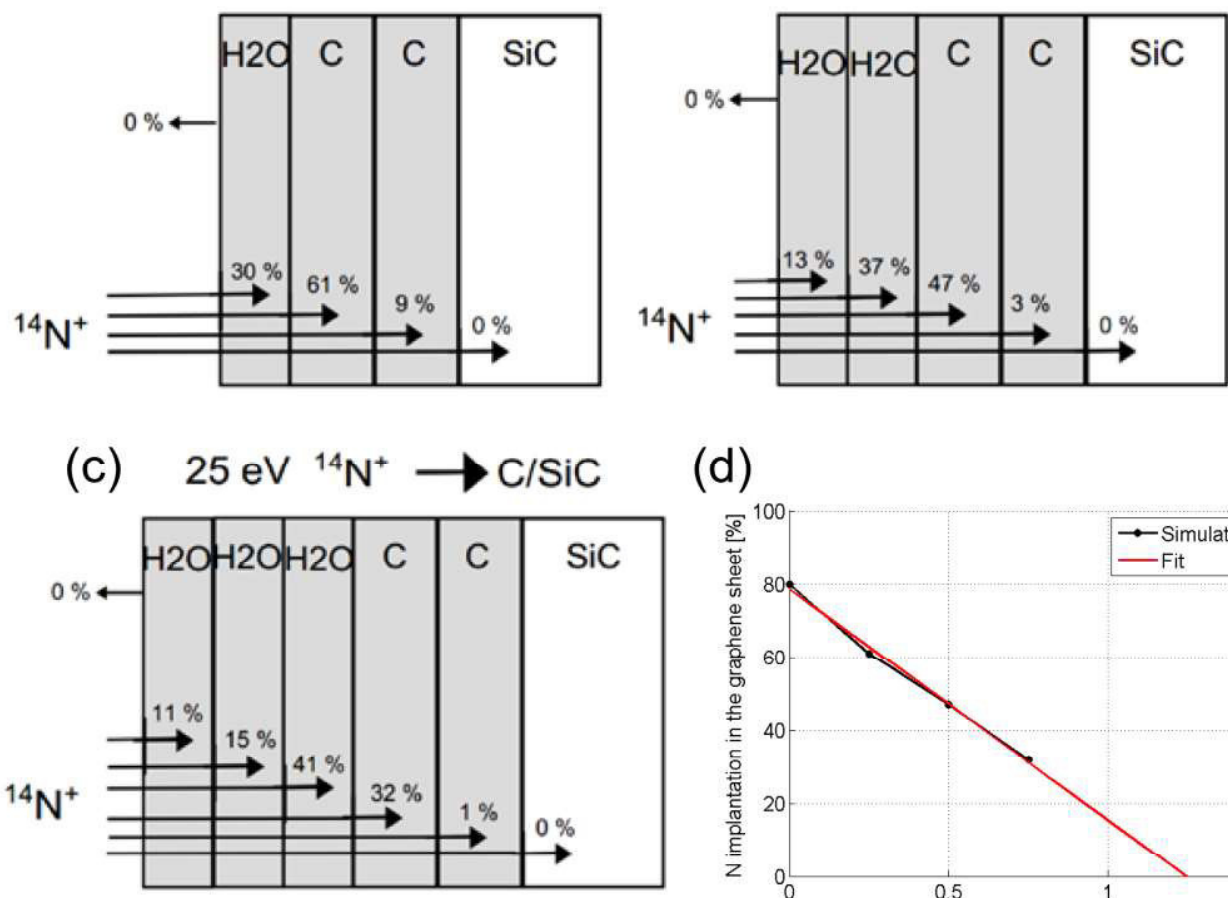

(d)

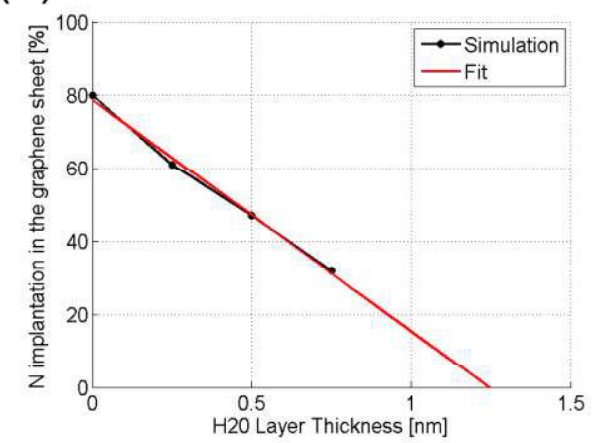

Figure 4.7: Influence on the incorporation for different adlayer thickness on top of the ML graphene sheet. 


\title{
4.3 Doping of Graphene by Low-Energy Ion Beam Im- plantation: Structural, Electronic, and Transport Properties
}

\author{
P. Willke, J. A. Amani, A. Sinterhauf, S. Thakur, T. Kotzott, T. Druga, S. Weikert, \\ K. Maiti, H. Hofsäss and M. Wenderoth
}

Nano Letters 15 (8), pp 5110-5115, June 2015

DOI: 10.1021/acs.nanolett.5b01280

We investigate the structural, electronic, and transport properties of substitutional defects in SiC-graphene by means of scanning tunneling microscopy and magnetotransport experiments. Using ion incorporation via ultralow energy ion implantation, the influence of different ion species (boron, nitrogen, and carbon) can directly be compared. While boron and nitrogen atoms lead to an effective doping of the graphene sheet and can reduce or raise the position of the Fermi level, respectively, ${ }^{12} \mathrm{C}^{+}$carbon ions are used to study possible defect creation by the bombardment. For low-temperature transport, the implantation leads to an increase in resistance and a decrease in mobility in contrast to undoped samples. For undoped samples, we observe in high magnetic fields a positive magnetoresistance that changes to negative for the doped samples, especially for ${ }^{11} \mathrm{~B}^{+}$- and ${ }^{12} \mathrm{C}^{+}$-ions. We conclude that the conductivity of the graphene sheet is lowered by impurity atoms and especially by lattice defects, because they result in weak localization effects at low temperatures.

Tailoring the electronic properties of graphene is an important requirement for its application in future electronic devices. Essentially, the position of the Fermi level and a corresponding tuning of the number of charge carriers are one of the major challenges. To achieve doping in graphene different approaches have been explored, including adsorption of adlayers[161] and intercalation of atomic layers,[47, $146,163]$ but also a direct incorporation of foreign atoms into the graphene sheet.[8, 83, 88, 176, 191, 193, 198, 206, 216, 217] This direct substitution can be realized during the growth process as usually done for graphene grown by chemical vapor deposition (CVD).[216, 217] Besides other techniques[83, 191, 193] ion bombardment has been established as a suitable way, allowing doping of graphene on different substrates.[8, 198, 206] This doping technique has the advantage of being easily transferable to other dopant atoms and other two-dimensional atomic crystals in the context of van der Waals heterostructures[55] like boron nitride $(\mathrm{BN})$, tungsten disulfide $\left(\mathrm{WS}_{2}\right)$, and molybdenum disulfide $\left(\mathrm{MoS}_{2}\right)$. Moreover, it allows an easy control of the dopant concentration through the ion fluence. 
The influence of doping by atomic substitution on transport processes remains important for device physics: the presence of foreign atoms as well as the possible creation of additional defects hinders the electronic performance by introducing atomic scale scattering centers. Therefore, it is crucial to connect the electronic properties of doped graphene sheets with their microscopic structure and their behavior in transport experiments.

Here, we report a comparative study using scanning tunneling microscopy/spectroscopy (STM/S) as well as transport measurements to study SiC-graphene doped with nitrogen/boron atoms. In contrast to nitrogen-doped graphene, borondoping has rarely been studied up to now[217] especially for epitaxially grown graphene on SiC. Figure 4.8a shows an STM image of a pristine graphene sheet on $\mathrm{SiC}$. Besides the graphene lattice the $6 \times 6$-reconstruction is clearly visible.[100] In Figure $4.8 \mathrm{~b}$, we introduced substitutional boron atoms into the system that manifest in a pronounced electronic contrast. Besides their influence on doping, these defects change the local structural and electronic properties and serve as atomic scattering centers for electron transport. This becomes apparent in Figure 4.8c where we show the magnetoresistance (MR) of the two systems. While the MR for the undoped system increases for increasing magnetic field, it strongly decreases for the doped systems that we attribute to an additional contribution of weak localization.[123] This connection between the microscopic structure and the transport properties reveals the processes taking place for electron scattering at substitutional atoms and additional defects in graphene and are in the focus of this paper.

(a)

(b)
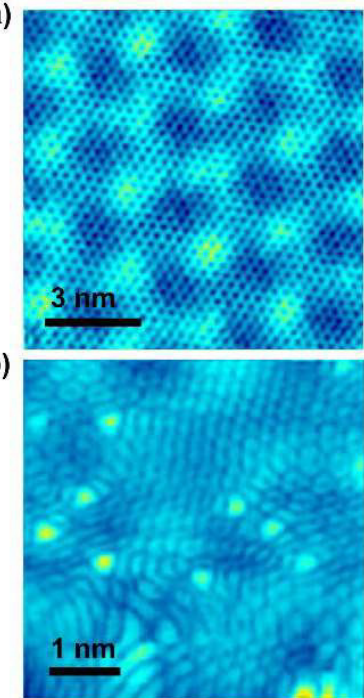

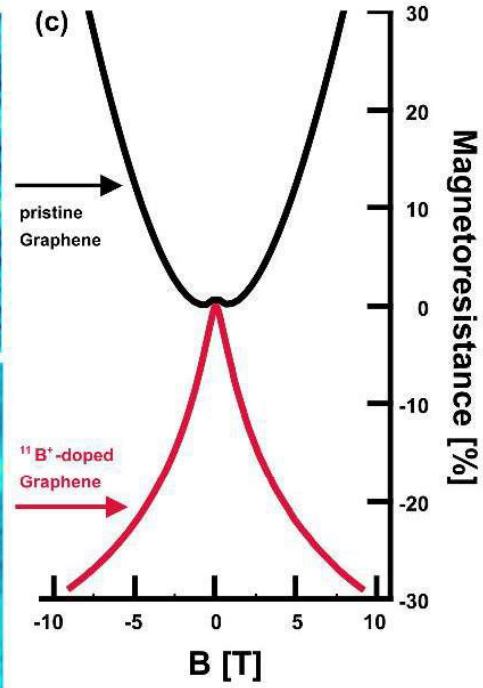

B [T]

Figure 4.8: Scanning tunnelling microscopy and magnetotransport of SiC-graphene samples. (a) Image of pristine (undoped) graphene on $\mathrm{SiC}(0001)$. The $6 \times 6$-reconstruction of the underlying SiC-lattice is clearly visible $\left(\mathrm{V}_{\text {Bias }}=0.05 \mathrm{~V}\right.$ and $\left.\mathrm{I}_{\text {set }}=250 \mathrm{pA}\right)$. (b) Graphene sample with additional single boron atoms ( $V_{\text {Bias }}=-50 \mathrm{mV}$ and $\left.\mathrm{I}_{\text {set }}=50 \mathrm{pA}\right)$ (c) MR with respect to its zero-field resistance. A clear transition from positive to negative MR is visible under the influence of doping. 
Our graphene samples have been prepared by thermal decomposition of $\mathrm{SiC}$.[155] Samples covered by mono- and bilayer graphene on the Si-terminated side are achieved by a heating step for 2 min at $1400-1600{ }^{\circ} \mathrm{C}$ in ultrahigh vacuum $(\mathrm{UHV})\left(<10^{-10} \mathrm{mbar}\right)$. After ex situ transfer to our mass selected ion beam deposition system, the respective ions are implanted into the graphene samples with a kinetic energy of $25 \mathrm{eV}$ and a fluence of $3 \cdot 10^{14} \mathrm{~cm}^{-2}$. Before the samples are inserted into our low-temperature STM, they are annealed for $30 \mathrm{~min}$ at temperatures between 350 and $450{ }^{\circ} \mathrm{C}$ in UHV. STM/S measurements were taken at $77 \mathrm{~K}$ (liquid nitrogen cooled) or at $6 \mathrm{~K}$ (liquid helium cooled). The details of the ion implantation technique can be found elsewhere.[8] For boron- and nitrogen-doped samples, the same samples have been used for transport experiments; for carbonimplanted and undoped samples the transport experiments have been conducted with different samples prepared in the same method and for the same fluences as for the STM data. Consequently, the carbon-implanted sample used in the transport measurements has also not been treated with the STM annealing step.

(a)

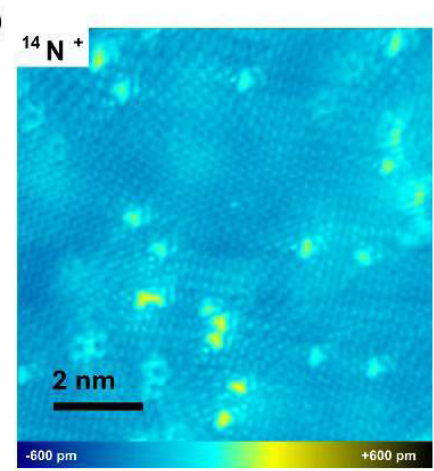

(d)

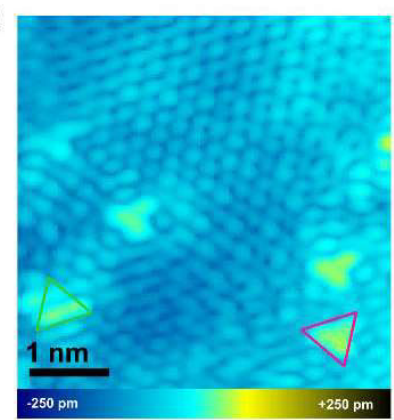

(b)

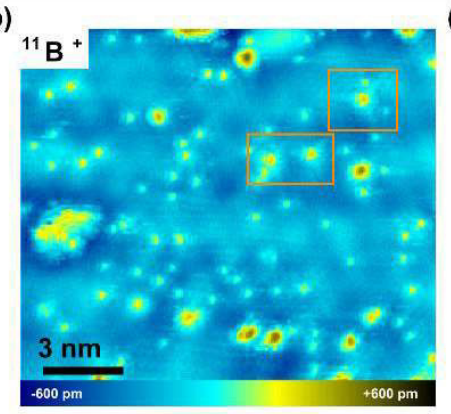

(e)

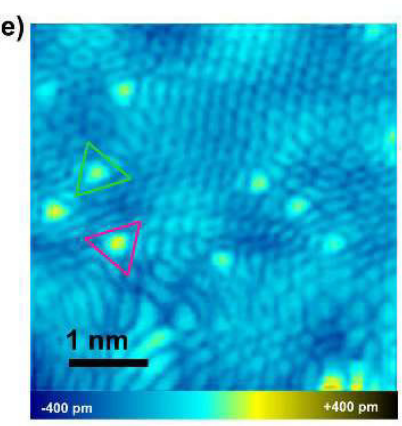

(c)

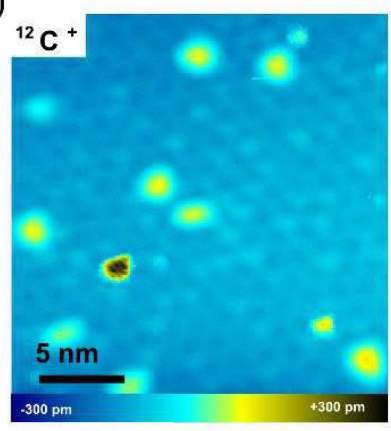

(f)

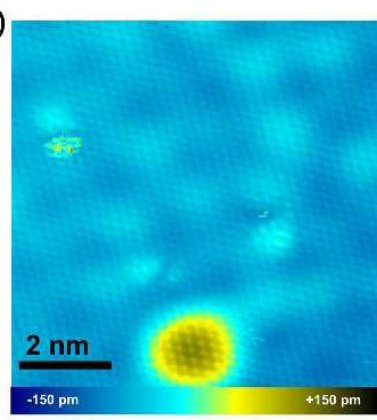

Figure 4.9: STM images of graphene with different dopant atom species incorporated by ultralow energy ion implantation. (a) Nitrogen-implanted sample $\left(V_{\text {Bias }}=-10 \mathrm{mV}\right.$ and $I_{\text {set }}=10 \mathrm{pA}$ ). The electronic contrast shows single graphitic-type nitrogen atoms. (b) Boronimplanted sample $\left(V_{\text {Bias }}=-0.3 \mathrm{~V}\right.$ and $\left.I_{\text {set }}=100 \mathrm{pA}\right)$. Besides the contrast of single graphitictype boron atoms one other contrast type is found [yellow boxes]. (c) Carbon-implanted sample $\left(V_{\text {Bias }}=0.3 \mathrm{~V}\right.$ and $\left.I_{\text {set }}=100 \mathrm{pA}\right)$. Defects are found on a larger scale and show different electronic contrasts. (d) Close-up of single nitrogen atoms $\left(\mathrm{V}_{\text {Bias }}=-20 \mathrm{mV}\right.$ and $\mathrm{I}_{\text {set }}$ $=20 \mathrm{pA})$ and $(\mathrm{e})$ single boron atoms $\left(V_{\text {Bias }}=-50 \mathrm{mV}\right.$ and $\left.\mathrm{I}_{\mathrm{set}}=50 \mathrm{pA}\right)$. Both sublattice orientations can be found (red and green triangles). (f) Carbon-implanted sample. Most common defects are topographic high regions with intact graphene layer (bottom) in addition to sharp atomic scale scatterers. $\left(V_{\text {Bias }}=0.3 \mathrm{~V}\right.$ and $\left.I_{\text {set }}=100 \mathrm{pA}\right)$. 
In Figure 4.9 we show constant current topography maps of all implanted atom types: nitrogen in Figure 4.9a, boron in Figure 4.9b, and carbon in Figure 4.9c with respective high-resolution images in Figure $4.9 \mathrm{~d}-\mathrm{f}$. In addition to the results by transmission electron microscopy (TEM)[8] and X-ray photoemission spectroscopy (XPS)[198] measurements, the triangular shape of the electronic contrast for both nitrogen and boron atoms supports a primarily substitutional implantation. Because there are two sublattices in graphene, the electronic contrast of the nitrogen and boron atoms can point into two different directions as indicated in Figure 4.9d,e. We find that both configurations are distributed equally consistent with observations from other implantation techniques, $[83,176]$ while sublattice domains have been found for nitrogen atoms in CVD-grown graphene.[212] The achieved dopant densities are $\left.n_{\text {nitrogen }}=(0.35 \pm 0.10) \mathrm{nm}^{-2} \quad \approx 0.9 \%\right)$ and $n_{\text {boron }}=(0.30 \pm$ $0.10) \mathrm{nm}^{-2}(\approx 0.8 \%)$ for nitrogen and boron, respectively. Using this technique about one-tenth of the ions become incorporated, most likely due to the presence of adsorbate adlayers and a too low kinetic energy of the ions.[8, 198] While we find several remains of adsorbates on both samples, only in boron-doped samples a second electronic contrast is found indicated by yellow boxes in Figure $4.9 \mathrm{~b}$ $\left[n_{\text {boron,defect }}=0.01-0.02 \mathrm{~nm}^{-2}(\approx 0.05 \%)\right.$ ]. Its symmetry is also three-fold but the extent of its electronic contrast is much greater in size. Because of its electronic signature and by comparison to carbon-implanted samples, it is most likely to be a vacancy (see Supporting Information in subsection 4.3.3.3). A carbon-implanted sample is shown in Figure 4.9c that serves as a reference system, because the carbon atoms only introduce lattice defects without impurity atoms and therefore take into account the influence of defect creation. Defect features are significantly less than for N/B indicating that the vast majority of $C$ ions performed a direct exchange with lattice atoms. However, more interesting are the defects that are now created in the graphene sheet. While untreated samples yield clean surfaces of more than $100 \mathrm{~nm}^{2}$ in size,[201] we here find several defects that range from sharp atomic scale contrasts to a few nanometers. Because we do observe a variety of atomic scale defects, we discuss them including their spectroscopic signature in the Supporting Information in subsection 4.3.3.3. The close-up in Figure 4.9f shows that the larger defects still reveal an intact graphene sheet on top. These "graphene hills" can be caused by intercalated carbon atoms during the implantation process. We do not expect this type of defect to influence the electron transport significantly; similar to a SiC-substrate step, the scattering might be influenced by the detachment from the substrate and the bending of the graphene sheet.[80] Nevertheless, both have been shown to be negligible concerning their influence on electron scattering. $[80,108]$ This is in agreement with the spectroscopy measurements on the defect (see Supporting Information in subsection 4.3.3.3) that hardly differ from the pristine graphene areas. However, another possibility for the occurrence of this 
defect type is the annealing of lattice defects with underlying displaced carbon atoms that originate from the irradiation process. This mechanism has recently been demonstrated [68] and implies that the graphene lattice is also broken previous to annealing. For the phase coherent transport, this latter explanation would be more severe, since then this defect type would also add to the number of atomically sharp scattering centers. Therefore, we here give the defect density $n_{\text {defect }}=$ $(0.013 \pm 0.007) \mathrm{nm}^{-2}(\approx 0.04 \%)$ excluding and $n_{\text {defect }}=(0.026 \pm 0.014) \mathrm{nm}^{-2}(\approx$ $0.08 \%$ ) including the graphene hills. The defect density in C-implanted samples is much smaller than the doping atom density for $\mathrm{B} / \mathrm{N}$-implanted sample. While in the boron-doped samples the density of the vacancies leads to a similar lattice defect density as for $\mathrm{C}$-implanted samples, the respective density observed in nitrogen is still much smaller; here, we hardly observe additional defects other than those shown in Figure 4.9a. Thus, the defect creation is depending on the ion species. This idea is supported by molecular dynamics simulations that found differences in implantation and defect creation rate for ion-implanted nitrogen and boron in graphene depending on the ions' kinetic energy.[2] Possible reasons are variations in the ion mass and the chemical interaction between the projectile and the recoil atom.

To discuss the effective doping by nitrogen and boron atoms and the local change in electronic structure in these samples we perform STS-measurements. The $\mathrm{d} / / \mathrm{d} V$-spectra for nitrogen- and boron-doped samples are presented in Figure 4.10. In Figure 4.10a, we show an averaged spectroscopy over positions of the pristine graphene sheet for the $\mathrm{N}$-doped case indicated by the solid line. The individual measurements at different positions on the sample are indicated by the single dots. Besides the pseudogap at $0 \mathrm{meV}$ [100] we observe a depletion at the Dirac point $E_{D}$. For undoped $\mathrm{SiC}(0001)$-graphene $E_{D}$ is found at around $-440 \mathrm{meV}$.[137] The corresponding measurement for boron-doped samples is shown in Figure 4.10b. For the nitrogen-doped [boron-doped] sample, we determine the position at $E_{D}=(-600 \pm 50) \mathrm{meV}\left[E_{D}=(+250 \pm 50) \mathrm{meV}\right]$ leading to a charge transfer of $0.35 \pm 0.11$ free electrons per $N$ dopant [0.63 \pm 0.25 holes per $B$ dopant] (see Supporting Information in subsection 4.3.3.3).

Besides the possibility of complementary doping of the graphene sheet with the same method, the spectroscopic properties at the doping atoms and their energetic features can be extracted. In Figure 4.10c,d, we show averaged d//dV-spectra of several nitrogen and boron atoms, respectively. For the nitrogen signature, the spectroscopic signal is depleted at negative bias voltage in agreement with others. $[83,176]$ Moreover, we find a strong increase for positive voltage indicating the onset of the nitrogen donor level.[83, 144] Similarly, for the boron signature in Figure $4.10 \mathrm{~d}$ a corresponding increase is found for negative bias voltages as expected 
for the boron acceptor level.[2] The total shape of the boron dopant spectrum fits theoretical predictions $[144,194]$ of the LDOS including the increase in spectral
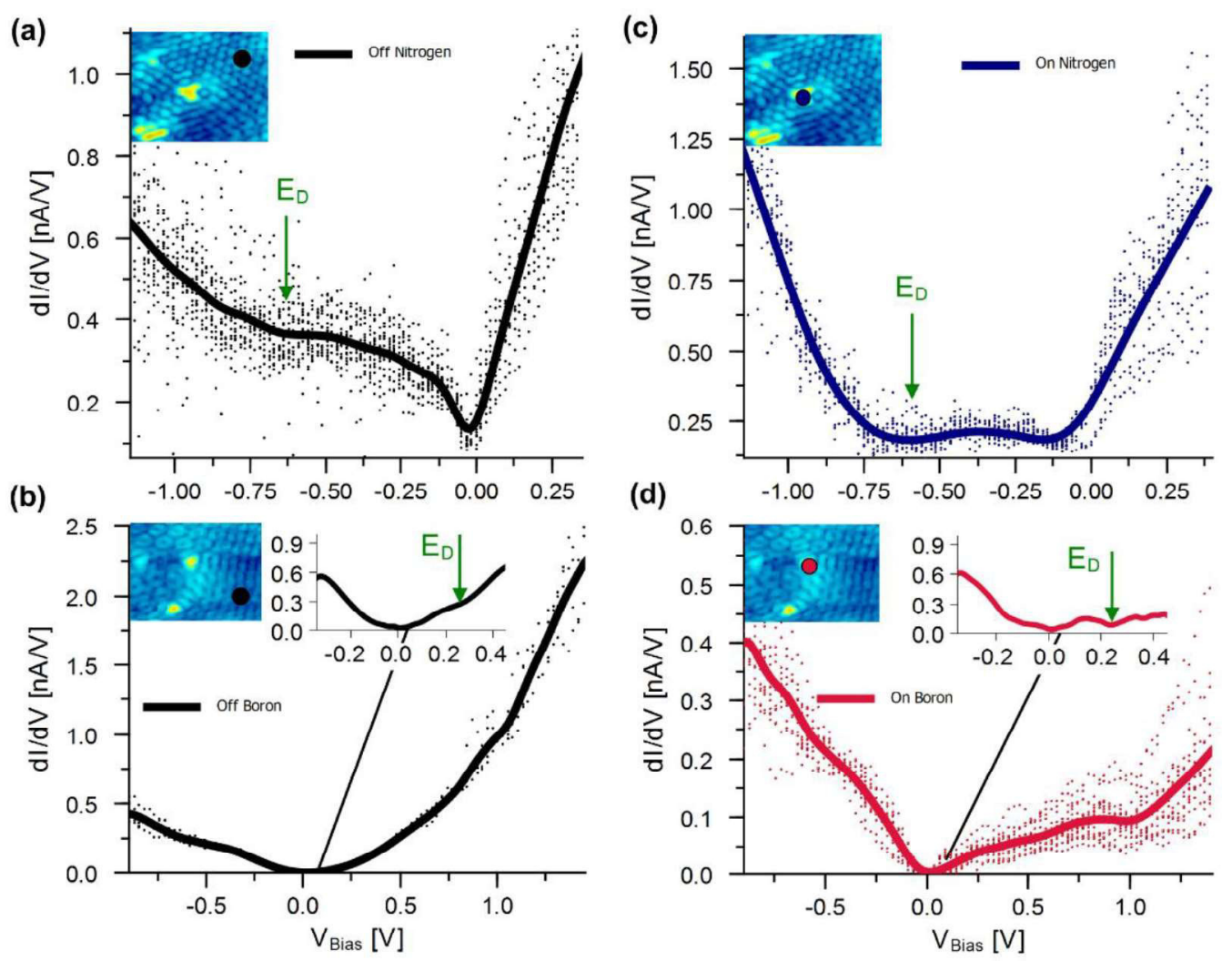

Figure 4.10: Scanning tunnelling spectroscopy of nitrogen- and boron-doped samples. (a) Spectroscopies of a nitrogen-doped sample on pristine graphene areas (see inset map) $\left(V_{\text {Bias }}=-1.2 \mathrm{~V}\right.$ and $\left.I_{\text {set }}=0.5 \mathrm{nA}\right)$. The position of the Dirac point $E_{D}=$ $(-600 \pm 50) \mathrm{meV}$ can be estimated from the depletion at negative bias voltage marked by the green arrow. At $0 \mathrm{~V}$, the pseudogap leads again to a depletion in conductance. (b) Spectroscopies of a boron-doped sample on pristine graphene areas $\left(V_{\text {Bias }}=-0.65 \mathrm{~V}\right.$ and $\left.I_{\text {set }}=0.09 \mathrm{nA}\right)$. Inset curve: Position of the Dirac point $E_{D}=(+250 \pm 50) \mathrm{meV} \quad\left(V_{\text {Bias }}=\right.$ $-0.4 \mathrm{~V}$ and $\left.I_{\text {set }}=0.12 \mathrm{nA}\right)$. (c) Spectroscopies on different nitrogen atoms $\left(V_{\text {Bias }}=-1.2 \mathrm{~V}\right.$ and $\left.I_{\text {set }}=0.5 \mathrm{nA}\right)$. (d) Spectroscopies on different boron atoms $\left(V_{\text {Bias }}=-0.65 \mathrm{~V}\right.$ and $I_{\text {set }}=$ $0.09 \mathrm{nA}$ ). The inset curve shows the position of the Dirac point $E_{D}$.

weight at $1 \mathrm{eV}$ that is depending on the interaction between neighboring sites.[144] For a high overlap parameter, we expect here also a quantitative agreement. From the given data, we calculated constant height spectra (see Supporting Information 4.3.3.2). These demonstrate that the LDOS is much larger on the defect leading to the topographic height contrast of the defects in agreement with others.[83]

To connect the electronic and structural properties of the doped SiC-graphene to its transport behavior we perform magnetotransport measurements. By introducing 
foreign atoms and defects, atomic scale scattering centers are induced that influence the phase coherent transport properties.[123] For the magnetotransport measurements, large area samples $(0.5 \mathrm{~mm})$ were contacted in 4-point geometry (van der Pauw method) in a shadow mask procedure. Experiments were conducted in a physical property measurement system (PPMS) equipped with a $9 \mathrm{~T}$ magnet. The results of the transport measurements can be found in Figure 4.11. As indicated in Figure $4.11 \mathrm{a}$, the sheet resistance $\rho$ at $4 \mathrm{~K}$ is significantly larger for the ion-implanted samples than for the undoped ones. The latter show a typical sheet resistance for SiC-graphene that is mostly limited to the steps and monolayer/bilayer-interfaces in our case and is therefore a little higher than comparable samples.[82] According to Drude's formula $\sigma=\left(e^{2} \tau n\right) / m^{*}=1 / \rho$, where $m^{*}$ is the effective mass of an electron and $\tau$ is the mean relaxation time, the sheet resistance depends on the charge carrier concentration $n$ making it biased by the doping level of the samples. To emphasize the influence of defect scattering only, we calculate the mobility $\mu$ shown in Figure $4.11 \mathrm{~b}$ using the charge carrier concentrations derived from STM data. The undoped samples show the highest mobility of $600-1000 \mathrm{~cm}^{2} \mathrm{~V}^{-1} \mathrm{~s}^{-1}$. For nitrogen- and boron-doped samples, the mobility is in the same order of magnitude of $90-110 \mathrm{~cm}^{2} \mathrm{~V}^{-1} \mathrm{~s}^{-1}$. This qualitatively agrees with a theoretical predicted decrease in mobility for nitrogen/boron doping in graphene due to impurity scattering.[194] The carbon-implanted sample exhibits a much smaller mobility of $\sim 10 \mathrm{~cm}^{2} \mathrm{~V}^{-1} \mathrm{~s}^{-1}$ along with a very high sheet resistance. This already demonstrates the prominent impact of lattice defects on transport properties in contrast to impurity atoms. In addition, the temperature-dependence in Figure 4.11c shows that this high resistance is vanishing for higher temperatures for all ion-implanted samples, while for boron and carbon implantation it is again more pronounced. The dependence on temperature for weak localization is expected to be $\Delta \rho \propto \ln \left(T_{0} / T\right)$.[1, 14, 152] While our temperature-dependent results show a good agreement with this model (see Supporting Information in subsection 4.3.3.5), a quantitative analysis is limited here by the onset of substrate conductivity and the overall number of data points. In Figure 4.11d, we present the MR in the range of $\pm 9 T$ for all ion types and for undoped samples. For high fields, we observe a positive MR for undoped samples as seen by others[152, 213] which here follows a $B^{2}$-dependence. Thus, we interpret this as a classical Lorentz MR.[152] This behavior changes to negative MR for ion-implanted samples, which is for undoped samples only found for small fields. Consequently, the presence of atomic scale defects leads to this change to negative MR. Interestingly, in the case of nitrogen implantation the change is much less than for boron and carbon, which could be explained with either a different scattering potential or the absence of lattice defects. The fact that for carbon-implanted samples the strong negative MR is observed without the presence of impurity atoms supports the idea that the 
change in MR is dominated by the additional lattice defects also present with boron-doping. Recently, a strong negative MR has also been observed for nitrogen implantation in contrast to the rather small negative MR in our samples.[152] Because their samples, which are characterized by Raman spectroscopy, possibly include other kinds of defects besides substitutional nitrogen, this result also fits to our hypothesis.

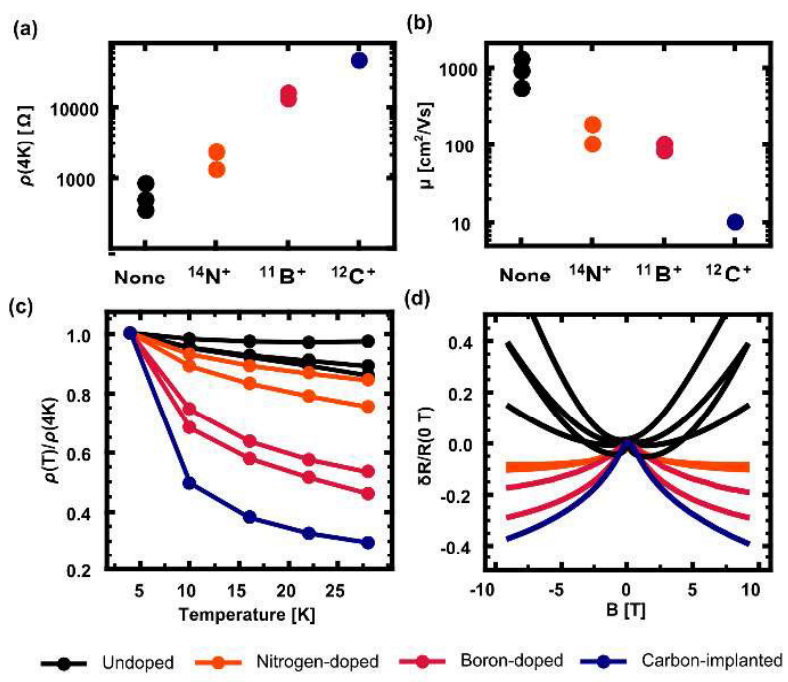

Figure 4.11: Transport measurements for pristine (undoped) and N-, B-, and C-implanted graphene. In total, eight samples have been analysed, represented by individual points/lines. (a) Sheet resistance $\rho$ and (b) mobility $\mu$ of all types of samples at $4 \mathrm{~K}$. (c) Temperature dependence of the sheet resistance for temperatures from 4 to $28 \mathrm{~K}$. (d) Magnetoresistance for high magnetic fields.

While a change of MR in graphene under the influence of defect creation has also been observed in other studies, $[73,219]$ the strong dependence on temperature and magnetic field indicates that the change in resistance is induced by phase coherent transport phenomena, that is, weak localization and weak antilocalization; atomically sharp scatterers lead to weak localization in graphene as shown theoretically.[123] This holds for the vacancies and impurity defects in ion-doped and carbon-implanted samples. By fitting the change in conductivity in the regime of $B= \pm 0.5 \mathrm{~T}$ as shown in Figure 4.12a to the theoretical description of weak localization[123] as done in studies with similarly prepared samples,[115] we can express the results in terms of intervalley scattering length $L_{i}$, phase coherence length $L_{\varphi}$, and the combined scattering length $L^{*}$ (see Supporting Information in subsection 4.3.3.4 for details on the analysis). The extracted values are shown in Figure 4.12b. Usually, weak localization is discussed for small magnetic fields < $1 \mathrm{~T}$. Strong negative MR in defective graphene has been explained in other works by diffuse scattering at grain boundaries[219] and magnetic polarons induced by vacancies[219] or magnetic adatoms.[73] In nitrogen-doped graphene[152] and 

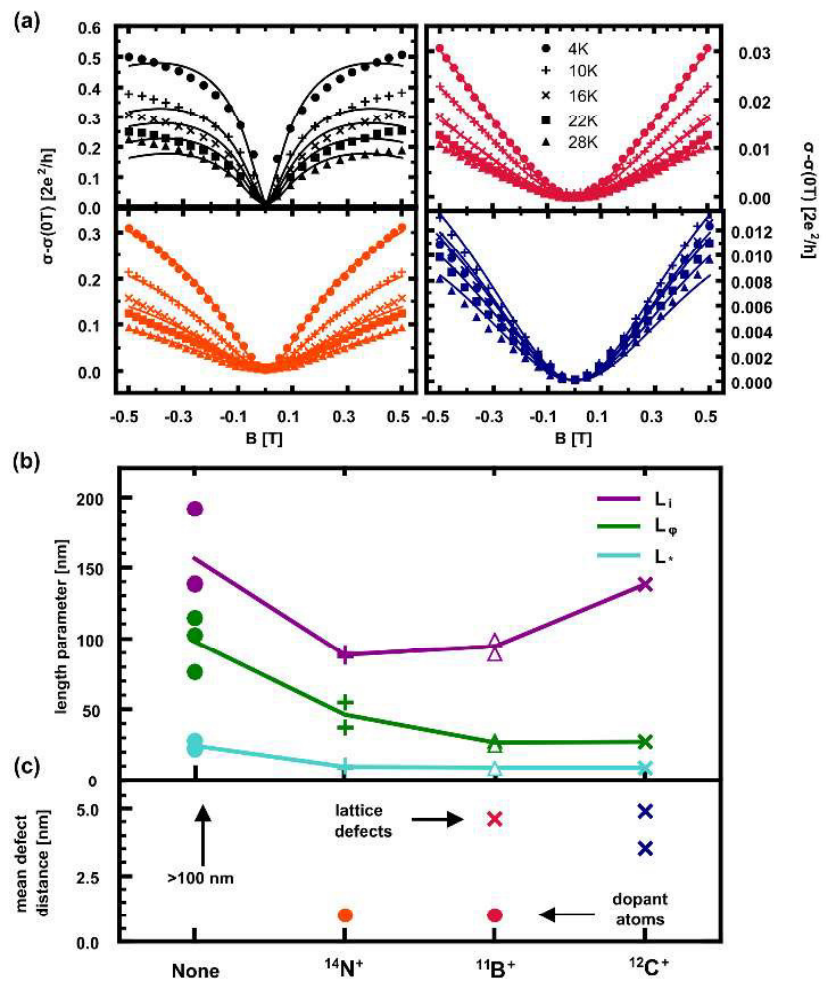

Figure 4.12: (a) Conductivity in the regime of small magnetic fields $(B< \pm 0.5 \mathrm{~T})$ with fits according to weak localization theory. For each sample type, only one sample is displayed. (b) Scattering lengths derived from the fits in (a): intervalley scattering length $L_{i}$, phase coherence length $L_{\varphi}$, and the combined scattering length $L_{*}$. (c) Mean defect distances derived from the STM topographies in Figure 4.9. While steps and interfaces are the only defects present for undoped samples, dopant atoms with a distance of approximately $1 \mathrm{~nm}$ can be found in nitrogen- and boron-doped samples. Boron- and carbon-implanted samples show additional lattice defects with approximately $5 \mathrm{~nm}$ mean distance. For carbon implantation, the data point with the higher mean defect distance is excluding the influence of the hill-defect type as described in the text.

graphite,[213] a superposition of weak localization and diffusive scattering in a magnetic field has been proposed to explain this strong negative MR. By considering the microscopic structure from the STM measurements, we can show that weak localization still can play a role in the high field regime: estimating the cyclotron radius $r$ via $B=\left(\varphi_{0} / 4 \pi r^{2}\right)$ where $\varphi_{0}$ is the fluxon for a magnetic field of $B=$ $9 \mathrm{~T}$ yields $r \approx 4.3 \mathrm{~nm}$. This is in the order of magnitude of the mean distance $L_{\text {mean }}=(\pi n)^{-1 / 2}$ between two defects as shown in Figure $4.12 \mathrm{c}$. In addition, the mean distance between defects is smaller than the extracted length parameters. Consequently, on average several defects lie within the range of the respective scattering process. For undoped samples, the results are comparable to similar studies[115] while we observe a higher intervalley scattering length. We find that all quantities decrease for the ion-implanted samples. While the intervalley 
scattering length is in all cases larger than the phase coherence length, it plays a minor role here. The combined scattering length yields the smallest values and is a factor of 2-3 smaller for ion-implanted samples. The phase coherence length is decreasing for the different ion types up to a factor of 4 . Certainly, this reduction is also influenced by a change of the diffusion constant $D$ that is connected by $L_{\varphi}^{2}=D \tau_{\varphi}$ and in this way effectively rescales the weak localization dependence (see Supporting Information in subsection 4.3.3.4). This can be seen by comparing Figure $4.12 \mathrm{a}, \mathrm{c}$. For $28 \mathrm{~K}$, where the influence of weak localization is almost vanished, we find a change in resistance $\rho(4 \mathrm{~K}) / \rho(28 \mathrm{~K}) \approx 3.3$ for the carbon samples and $\rho(4 \mathrm{~K}) / \rho(28 \mathrm{~K}) \approx 1.1$ for the undoped samples. On the contrary, the nominal resistance in Figure $4.12 a$ of both sample types differs by a factor of $\rho_{\text {carbon }}(4 \mathrm{~K}) / \rho_{\text {undoped }}(4 \mathrm{~K}) \approx 50-100$. Thus, there is still a contribution from diffusive scattering manifested in a different diffusion constant $D \propto 1 / \rho$.

The microscopic structure of the nitrogen-implanted samples showed no additional lattice defect creation besides the substitutional atoms in contrast to boron and carbon implantation. For the latter, it is evident from the transport measurements that a reduction of lattice defects is necessary to improve the conductivity of the graphene sheet. A possible method to eliminate unwanted defects is an additional high-temperature annealing step that can change the microscopic structure for doped graphene depending on annealing temperature and time.

In conclusion, we investigated the structural, electronic, and transport properties of ion-implanted atoms in SiC-graphene, namely boron, nitrogen and carbon. We showed that the incorporation with boron and nitrogen leads to an effective doping of the graphene sheet. Moreover, the doping leads to higher resistance, a lower mobility, and suppresses phase coherent transport. This is mediated by the impurity potential of the doping atoms, but more importantly by atomic scale defects that are created during the ion-implantation process. We demonstrate that this influence can be distinguished by the combination of STM and transport measurements. The method of low-energy ion implantation offers an alternative to chemical doping in graphene and is compatible to commercial semiconductor technologies. Moreover, it triggers new prospects for tailoring the properties of graphene by introducing metallic, magnetic, or rare earth elements.

\subsubsection{Author Contributions}

M.W., P.W., H.H., and J.A.A planned the experiments. P.W., S.T., T. D., and T. K. performed the STM measurements. J.A.A., H.H., and S.W. performed the ion implantation of the samples. A.S. and P.W. performed the transport measurements. The manuscript was written through contributions of all authors. All authors have given approval to the final version of the manuscript. 


\subsubsection{Acknowledgement}

This work was supported by the Deutsche Forschungsgemeinschaft (DFG) priority program 1459 Graphene. Financial support by the DFG under Contract No. HO1125/21-1 was also acknowledged. The authors S.T. and K.M. acknowledge financial support from India office, Georg August University, Göttingen, under the academic cooperation between TIFR, Mumbai and Georg August University, Göttingen. The authors thank the I. Physikalisches Institut, Georg-August-Universität Göttingen for using their physical property measurement system to conduct the transport measurements.

\subsubsection{Supporting Information}

\subsubsection{Evaluation of charge carrier concentration by Scanning Tun- neling Spectroscopy}

For the nitrogen-implanted sample we determine the position of the Dirac point in Figure 4.10a in the manuscript at $E_{D}=(-600 \pm 50) \mathrm{meV}$. From this we can derive the charge carrier concentration by $n=E_{D}^{2} / \pi\left(\hbar v_{F}\right)^{2}$ with the Fermi velocity $v_{F} \approx$ $1 \cdot 10^{6} \mathrm{~m} / \mathrm{s}$ [117]. Thus, we obtain a total charge carrier concentration of $n_{N}=2.6$. $10^{13} \mathrm{~cm}^{-2}$ in contrast to the undoped case where $n_{0}=1.4 \cdot 10^{13} \mathrm{~cm}^{-2}$. This leads to a doping of $0.35 \pm 0.11$ free electrons per $\mathrm{N}$ dopant which is comparable to results obtained by Zhao et al.[216] In case of the boron-doped sample in Figure $4.10 \mathrm{~b}$ one would assume that the position of the Dirac point is shifted towards positive bias voltages. While it is hardly visible in the spectroscopy with a large voltage range, we identify a depletion in the inset that is focusing around small positive voltages. We find a position of $E_{D}=(+250 \pm 50) \mathrm{meV}$. The respective total charge carrier concentration of $n_{B}=0.5 \cdot 10^{13} \mathrm{~cm}^{-2}$ is now obtained along with a change from $n$ - to $p$-doped graphene. The doping of $0.63 \pm 0.25$ holes per $B$ dopant is larger than for nitrogen doping, but still comparable to results obtained by Zhao et al.[217] Reasons for the deviation are uncertainties in the position of the Dirac point and the number of dopant atoms determined by STM which has both been included in the error calculation.

\subsubsection{Constant height spectroscopies of single boron and nitrogen atoms}

In Figure 4.13 we show reconstructed constant height spectra for the boron and nitrogen measurements shown in Figure 4.10 with respect to the respective clean graphene areas. This has been determined by additional local barrier height measurements that reveal a change in measured barrier height on the defect. Therefrom a constant height map can be calculated via the exponential decay of the tunneling current with increasing distance.[53] This demonstrates that the LDOS is much higher on the dopants than on the graphene sheet for constant tip-sample distance. 
It leads to the strong topographic contrast of the doping atoms in agreement with others.[83]
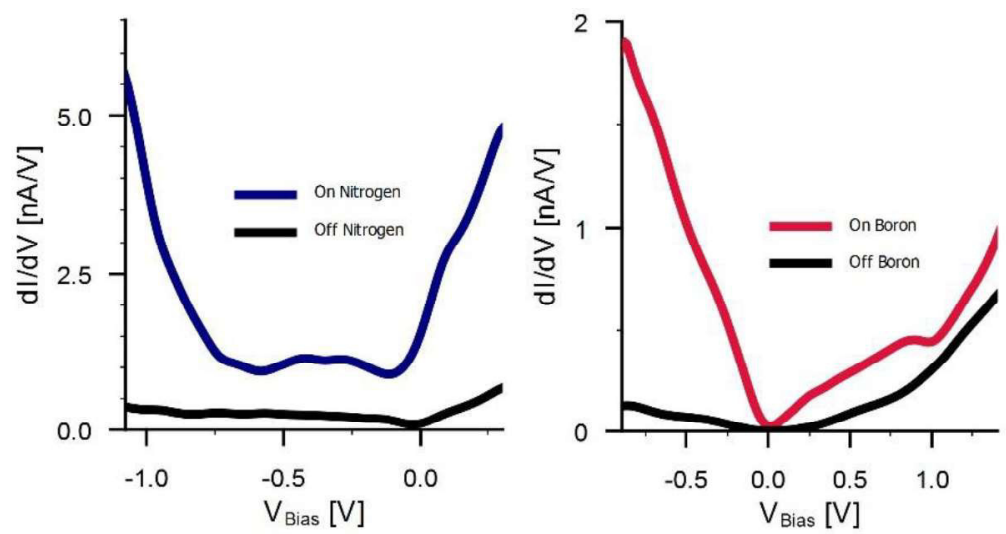

Figure 4.13: Calculated spectra in constant height mode derived from the spectroscopies shown in Figure 4.10.

\subsubsection{Defects in boron- and carbon-implanted samples}

While the predominant effect of the ion bombardment is a direct graphitic substitution of carbon by the dopant atoms, in general ion bombardment can generate other defects $[8,88]$ which are in most cases unwanted byproducts. In case of boron-doped samples we find a second electronic contrast shown in Figure 4.14a. Figure $4.14 \mathrm{~b}-\mathrm{g}$ display several defect types that are found in the carbon-implanted sample. Both defects in Figure 4.14a and Figure 4.14b that are taken on the boronand carbon-implanted sample, respectively, show a strong spectroscopic feature at $475 \mathrm{mV}$ (boron) and $75 \mathrm{mV}$ (carbon) which lie above the Dirac point for both systems. A similar strong resonance above the Dirac point has been observed for vacancies in graphene on $\mathrm{Pt}(111)$.[182] Therefore, we attribute these structures to vacancies in the graphene lattice. The graphene hill defect in Figure 4.14c shows besides a larger pseudo-gap no spectroscopic feature compared to the graphene sheet, which reflects the intact graphene layer on top that keeps its electronic properties. The defect in Figure 4.14d shows a shift of the Dirac point from $-350 \mathrm{mV}$ to $-280 \mathrm{mV}$. Since for increasing numbers of graphene layers, the Dirac point in $\mathrm{SiC}(0001)$-graphene is shifted towards higher energies due to a vanishing influence of substrate doping, $[100,137]$ this defect could reflect the formation of a partial new layer under the current graphene sheet. Even more defects are found and depicted in Figure 4.14e-g that show different electronic contrasts than the graphene sheet. However, without further input from theory or experiment their electronic pattern and spectroscopic signature do not lead to an unambiguous conclusion for the structure of the defect. 
(a)
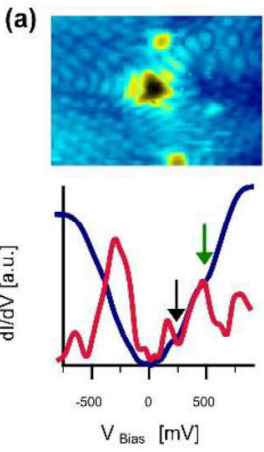

(e)
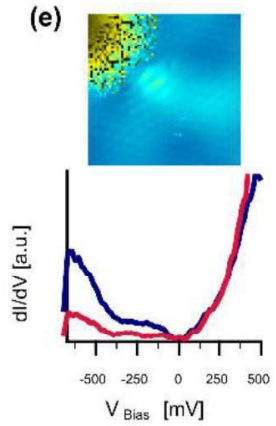
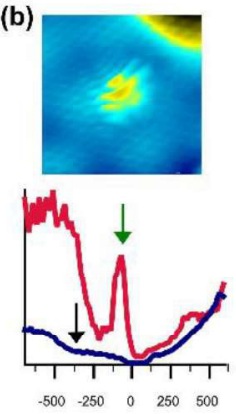

$\mathrm{V}_{\text {Bias }}[\mathrm{mV}]$

(f)

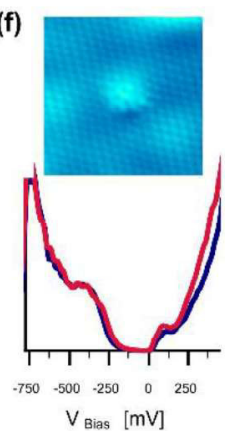

(c)
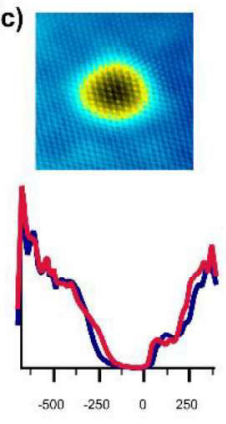

$V_{\text {Bias }}[\mathrm{mV}]$

(g)

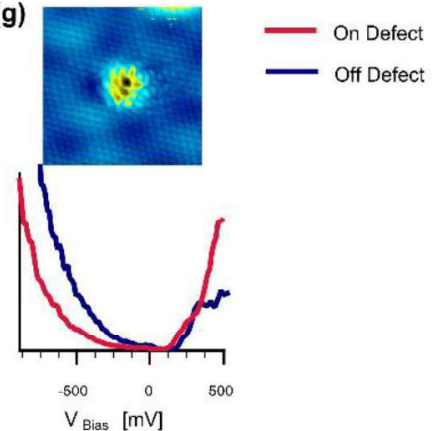

Figure 4.14: Defect formation in graphene samples. Blue lines represent the $\mathrm{dl} / \mathrm{dV}$-signal in the defect-free region near the respective defect. The red line is the same signal on the defect. (a) Defect-type found in boron-doped samples. Black arrow indicates the position of the Dirac point, green arrow the position of a high spectroscopic resonance above the Dirac point. As explained in the text, we assume this to be a vacancy. (b)-(g) Defect-types in carbon implanted samples. (b) is again assumed to be a vacancy with the Dirac point indicated by the black arrow and the spectroscopic resonance in green. Imaging conditions: (a) $-0.3 \mathrm{~V} / 80 \mathrm{pA}$ (b) $-0.15 \mathrm{~V} / 120 \mathrm{pA}$ (c) $0.6 \mathrm{~V} / 100 \mathrm{pA}$ (d) $0.04 \mathrm{~V} / 100 \mathrm{pA}$ (e) $0.5 \mathrm{~V} / 200 \mathrm{pA}$ (f) $0.3 \mathrm{~V} / 100 \mathrm{pA}(\mathrm{g}) 0.5 \mathrm{~V} / 100 \mathrm{pA}$

\subsubsection{Details on the fitting of the magnetotransport data}

In order to obtain the intervalley scattering length $L_{i}$, the phase coherence length $L_{\varphi}$, and the combined scattering length $L_{*}$ from the change in conductivity in the regime of $B= \pm 0.5 \mathrm{~T}$ the theoretical description of weak localization in graphene[123] is used to analyze our data. As done by Mahmood et al.[115] the change of conductivity in the regime of small magnetic fields can be described as

$$
\delta \mathrm{G}=\frac{e^{2}}{\pi \hbar}\left[F\left(\frac{B}{B_{\phi}}\right)-F\left(\frac{B}{B_{\phi}+2 B_{i}}\right)\right]-\frac{2 e^{2}}{\pi \hbar} F\left(\frac{B}{B_{\phi}+B_{i}+B_{*}}\right)
$$

where the function $\mathrm{F}$ is defined as $F(x)=\ln (x)+\Psi\left(\frac{1}{2}+\frac{1}{x}\right)$ and $\Psi$ is the digamma function.

The magnetic fields $B_{\varphi, i, *}$ are related to different relaxation times $\tau_{\varphi, i, *}$ using $B_{\varphi, i, *}=$ $\Phi_{0} / 4 \pi D \tau_{\varphi, i, *}$. 
In this equation $\Phi_{0}$ is the magnetic flux quantum and $D$ the diffusion constant. $\tau_{\varphi}$ is the phase coherence time, $\tau_{i}$ the intervalley scattering. The combined scattering time $\tau_{*}$ includes the intervalley scattering time, the intravalley scattering time $\tau_{z}$ and a correction due to trigonal warping $\tau_{w}$. It is defined as $\tau_{*}^{-1}=\tau_{i}^{-1}+\tau_{z}^{-1}+$ $\tau_{w}^{-1}$.[123] Length parameters are obtained using the relation $L_{\varphi, i, *}^{2}=D \tau_{\varphi, i, *}$, respectively, leading to $B_{\varphi, i, *}=\Phi_{0} / 4 \pi L_{\varphi, i, *}^{2}$. [115] Therefore, we do not need to express the diffusion constant $D$ explicitly, since we want to compare the length scale of the scattering processes with the microscopic density of the scatterers. However, it is also changing as can be seen from the sheet resistances in Figure 4.11. In order to estimate the sensitivity of the model with respect to the obtained scattering lengths each parameter has been varied until it fails to describe the experiment. We find that the theoretical model is the least sensitive to variations of the combined scattering length with an uncertainty of about $30 \%$. In contrast, only small uncertainties for the phase coherence length and the intervalley scattering length have been obtained being in the range of about $5 \%$.
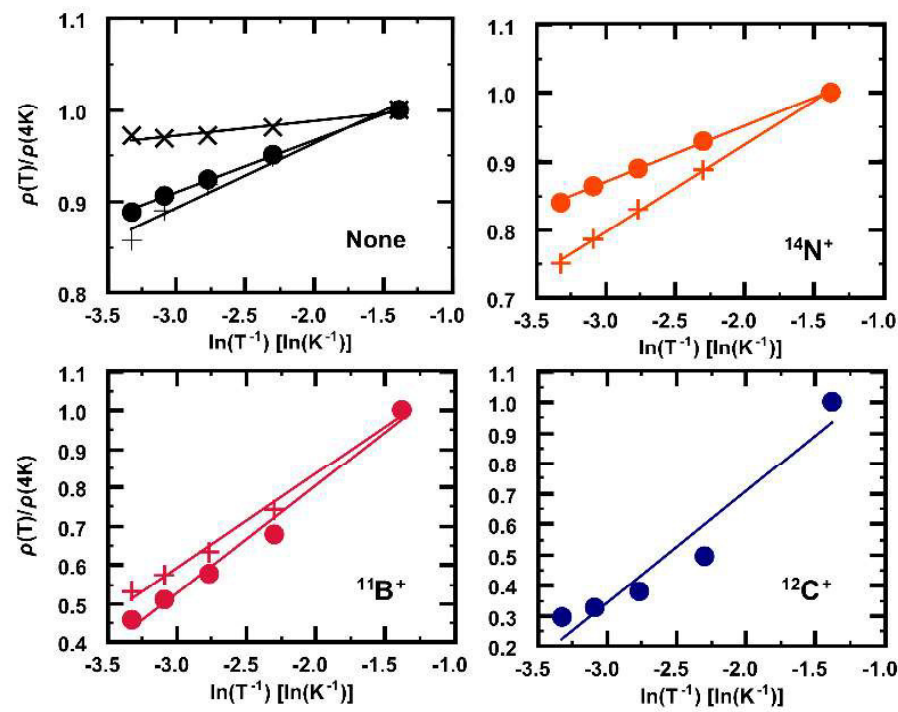

Figure 4.15: Temperature dependent normalized sheet resistance from $4 \mathrm{~K}$ to $28 \mathrm{~K}$ for undoped, nitrogen-doped, boron-doped and carbon-implanted graphene. Here, we plotted the resistivity against $\ln (1 / T)$ to pronounce the agreement with weak localization theory, for which a linear behavior is expected.

\subsubsection{Temperature dependence of the magnetotransport data}

In this section we discuss in detail the temperature dependence of the sheet resistance as shown in Figure $4.11 \mathrm{C}$ in the manuscript. In a 2D system the change due to weak localization is expected to be $\Delta \rho \propto \ln \left(T_{0} / T\right)$. $[1,14,152]$ In Figure 4.15 we show the temperature dependence of the (normalized) resistivity of all types of samples, evaluated to show a linear behavior for the weak localization dependence. We find straight lines for undoped samples as well as for nitrogen doping. While for boron doping the fit is still good, there are some deviations for carbon 

and Transport Properties

implantation. This supports the idea that doping atoms and lattice defects have a different impact on the transport process. The small deviation for carbon could origin from other scattering contributions discussed in literature and in the manuscript. $[73,152,219]$ However, given the small number of data points and the limited temperature range due to the onset of conductivity of the substrate the agreement with theory is quiet well.

In the same framework of temperature dependence of weak localization[1], the evolution of the phase coherence length with temperature can be evaluated from the magnetotransport measurements. In contrast to the intervalley scattering length $L_{i}$ and the combined scattering length $L_{*}$, the phase coherence length $L_{\varphi}$ is the only one assumed to be temperature dependent.[115] The temperature dependence of $L_{\varphi}$ is shown in Figure 4.16a. We find that it decreases with increasing temperature for all types of samples. For the carbon implanted sample the phase coherence length decays very slowly up to $16 \mathrm{~K}$, but for higher temperatures we find the same behavior as for the other sample types. Undoped and nitrogen-doped samples exhibit a qualitatively similar and much stronger decrease, which matches with the larger change in MR for boron-doped and carbon-implanted samples. According to [1] and [204] a linear dependence $L_{\varphi}^{-2} \propto T$ is expected for low temperatures, which is mostly fulfilled by our data, as depicted in Figure 4.16b. The mean extracted slope for the undoped samples is $4.5 \times 10^{12} \mathrm{~m}^{-2} \mathrm{~K}^{-1}$. It is in the same order of magnitude as the slope determined by others [115]. For nitrogen and boron we obtain the same mean slope of $2.5 \times 10^{13} \mathrm{~m}^{-2} \mathrm{~K}^{-1}$ being larger than the value extracted for the carbon implanted sample of $7.5 \times 10^{12} \mathrm{~m}^{-2} \mathrm{~K}^{-1}$.
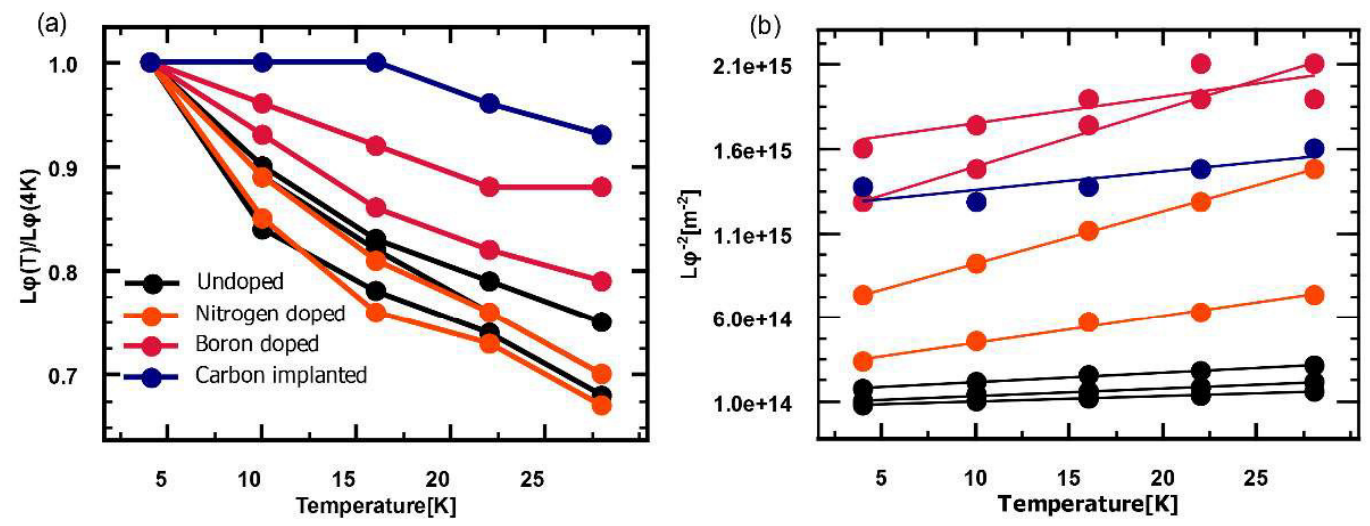

Figure 4.16: Temperature dependence from $4 \mathrm{~K}$ to $28 \mathrm{~K}$ for doped and defective graphene of (a) the phase coherence length $L_{\varphi}$ and (b) $L_{\varphi}^{-2}$ with corresponding linear fits. 


\section{Discussion}

In the following, we want to discuss the results of the preceding chapters and highlight the main aspects. By comparison with results of the same and other techniques, we will create a more general picture of electron scattering at localized defects and their influence on transport. Additionally, we will discuss the prospects and challenges for substitutional doping. At last, an outlook is given for future experiments. ${ }^{23}$

\subsection{Transport properties of extended defects}

\subsubsection{Defects in graphene on $\mathrm{SiO}_{2}$}

In section 3.3 two different types of defects have been discussed for graphene on $\mathrm{SiO}_{2}$ : grain boundaries and wrinkles.

For the defect resistance of wrinkles in graphene on $\mathrm{SiO}_{2}$ we show different values from literature in Table 5.1. While the only other experimentally obtained value suggests a wrinkle resistance of $200 \Omega \mu \mathrm{m}$ we found lower values in section 3.3 (Figure 3.18). Certainly, the wrinkle defect resistance is influenced by a variety of parameters: its width, the change in doping across the wrinkle, its curvature, as well as if it is a collapsed wrinkle allowing interlayer tunneling or not [203, 220]. The lowest detected value for a wrinkle is however measured by us for graphene on $\mathrm{SiC}$ and is further discussed in the next subsection. Thus, the presence of a wrinkle itself is not necessary having a high impact on transport. Additionally, we found in section 3.3 that folded collapsed wrinkle show a higher resistance than unfolded standing wrinkles. Here, we believe that interlayer tunneling is dominating the transport through the wrinkle. This has been suggested by Zhu et al. theoretically [220] and supported by the evidence of temperature-independent resistance at the defect.

Grain boundaries cannot be structurally identified using our AFM/KPFM technique. The size of a single grain however is much smaller than the transport maps shown in section 3.3, Figure $3.15^{24}$ and therefore is certainly included and measured.

${ }^{23}$ Parts of this chapter were later published in a review article (Willke et al. Electronic Transport Properties of 1D-Defects in Graphene and Other 2D-Systems. Annalen der Physik (Berlin), 529, 1700003, 2017).

24 The size of a single grain is usually in the order of a few $\mu \mathrm{m}$ (personal communication with the manufacturer - graphene supermarket). 
Since the largest defect resistance observed in this study is $80 \Omega \mu \mathrm{m}$, we take this as an upper limit for the grain boundary resistance ${ }^{25}$. A huge span of values is found in recent studies ranging from $8-43000 \Omega \mu \mathrm{m}$ (a summary is given in Ref. [61] and also in Figure 5.2), which can be explained with different misalignment angles of graphene patches, the total grain boundary width [30] as well as the quality of the latter itself, induced by the growth method [181]. Given this variation we find it worth noting that state-of-the-art commercial samples as used in our study do not show a significant influence by grain boundaries on electron transport.

\begin{tabular}{|c|c|c|c|c|}
\hline Publication & System & Defect & $\begin{array}{c}\text { Defect re- } \\
\text { sistance } \\
\rho_{\text {Defect }}[\Omega \mu \mathrm{m}]\end{array}$ & Method \\
\hline Clark et al. & Graphene on $\mathrm{SiO}_{2}$ & Wrinkle, $20 \mathrm{~nm}$ & 200 & STP \\
\hline Zhu et al. & Graphene & Folded Wrinkle & $200 \pm 150$ & NEGF \\
\hline Zhu et al. & Graphene on $\mathrm{SiO}_{2}$ & Folded Wrinkle & No influence & Transport \\
\hline Yan et al. & $\begin{array}{c}\text { Functionalized } \\
\text { Graphene on } \mathrm{SiO}_{2}\end{array}$ & Wrinkle & No influence & KPFM \\
\hline Willke et al. & Graphene on $\mathrm{SiO}_{2}$ & $\begin{array}{c}\text { Wrinkle, Folded } \\
\text { Wrinkle }\end{array}$ & $25-80$ & KPFM \\
\hline Willke et al. & Graphene on SiC & $\begin{array}{c}\text { Wrinkle, } 10-20 \\
n m\end{array}$ & $6.9 \pm 2.1$ & STP \\
\hline
\end{tabular}

Table 5.1. Defect resistance for wrinkles in graphene. Data taken from Ref. [30, 208, 220] as well as section 3.3 and 3.4. In the studies by Yan et al. and Zhu et al. the influence of the wrinkle was below their resolution.

\subsubsection{Defects in SiC-graphene}

In Table 5.2 we show values for the resistance of all types of extended defects in graphene on $\mathrm{SiC}$ that have been analyzed locally up to now. ${ }^{26}$ Our results are in good agreement with those obtained previously by Ji et al. [80]. Along with theoretical calculations using the nonequilibrium Green's function method [108], a linear increase of the resistance with $\mathrm{SiC}$-step height was proposed, which also leads to a good agreement for the small SiC-substrate step. Here, we find for the smallest

\footnotetext{
25 This value has been measured for a folded graphene wrinkle. Though there is no experimental evidence that this defect type involves an additional grain boundary [220], we take this value as the upper limit, since it is the largest localized defect resistance observed.

${ }^{26}$ Similar values have been obtained for the steps and MLBL-interfaces in our group by Druga [42]. The analysis of local resistances however stands out in his work in comparison to the other studies, since the values have been obtained with a different evaluation method, that is by full 2D resistornetwork simulations. Therefore, we here only state our results (Willke et al. [202], section 3.4, Table 3.4) obtained in the same manner as previous studies, that is, by direct evaluation of the voltage drop found in STP as described in subsection 1.2.3.
} 
possible step height ( $1 \mathrm{SiC}$ bilayer, $250 \mathrm{pm}$ ) roughly half the value compared to a double step ( $500 \mathrm{pm}$ ) obtained by Ji et al. Additionally, also larger steps (3/4 SiCbilayer) are in reasonable agreement while the $\mathrm{ML} / \mathrm{BL}$-interface agrees quite well with the value stated by Ji et al. For graphene bilayer wrinkles we obtained the first results up to now. As discussed in subsection 3.4.3 we propose a similar scattering mechanism as for the SiC-substrate step. Here, it was argued that a detachment from the substrate and buffer layer changes the doping level that both cause strong $\mathrm{n}$-doping in the graphene sheet $[80,108]$. Thus, a potential barrier is induced. The higher value compared to the substrate step can be explained by the larger extent (up to $20 \mathrm{~nm}$ ) of the wrinkles.

While the good agreement of different studies helps to quantify the results, a crucial difference between the experiments - the sample temperature - allows to further characterize the scattering mechanism at the defects. While $\mathrm{Ji}$ et al. conducted their experiments at room temperature and liquid nitrogen temperature (Clark et al.: liquid nitrogen temperature), our experiments have been conducted at liquid helium temperature $(6 \mathrm{~K})$. Consequently, the defect resistance stays constant over

\begin{tabular}{lcc} 
Publication & \multicolumn{1}{c}{ Defect } & $\begin{array}{c}\text { Defect resistance } \\
\boldsymbol{\rho}_{\text {Defect }}[\Omega \mu \mathrm{m}]\end{array}$ \\
Ji et al. & SiC-substrate step $(500 \mathrm{pm})$ & $8.0 \pm 3.0$ \\
Clark et al. & SiC-substrate step $(250 \mathrm{pm})$ & 1.2 \\
Willke et al. & SiC-substrate step $(250 \mathrm{pm})$ & 3.6 \\
\hline \hline Ji et al. & SiC-substrate step $(1.0 \mathrm{~nm})$ & $15.8 \pm 3.5$ \\
Willke et al. & SiC-substrate step $(0.75 \mathrm{~nm})$ & 23.4 \\
\hline \hline Ji et al. & ML/BL-interface $($ with $500 \mathrm{pm} \mathrm{step})$ & $21.6 \pm 5.6$ \\
& & $(29.3 \pm 6.9)$ \\
Clark et al. & ML/BL-interface $($ with $1 \mathrm{~nm} \mathrm{step})$ & $1.4-1.9$ \\
& & $(77 \pm 90)$ \\
Willke et al. & ML/BL-interface & $20.6 \pm 7.5$ \\
\hline \hline Willke et al. & Wrinkle & $6.9 \pm 2.1$
\end{tabular}

Table 5.2. Defect resistance for defects in graphene on SiC. Data taken from Ref. [80] and [30] as well as from chapter 3.

a wide range of temperature and thus we find strong indications that the underlying scattering mechanisms are independent of it. This is again - similar to the independence of defect resistances on magnetic field - in contrast to the graphene sheets that show a change in resistance with temperature by a factor of 2 between 4-300 K [25, 82]. Consequently, we find that the defect resistances behave as 


$$
\rho_{\text {Defect }}(B, T)=\rho_{\text {Defect }}
$$

and the underlying scattering mechanism should fulfill these independencies. The results gained within the scope of this thesis for structural defects in graphene, in particular the spatially resolved voltage drop (section 3.2), the magnetic field analysis (section 3.3) as well as the low temperature defect resistance measurements (section 3.3), rule out certain sources of scattering to be dominant $[86,95,105,126,150]$. For example, weak localization, which was found to contribute significantly in case of the substitutional atoms in section 4.3, would lead to a strong decrease of the defect resistance with B-field and would also be present only at low temperatures, therefore inducing a temperature-dependent resistance. Such a Bfield dependent behavior has been indeed observed for graphene grain boundaries in Ref. [210]. For the structural defects in SiC-graphene we however do not observe a decrease with $B$ and therefore can exclude this scattering mechanism here.

Tunneling has already been discussed in section 3.3 of chapter 3 in case of folded wrinkles which showed evidence for temperature-independent scattering. The tunneling between layers is only influenced in temperature $T$ by changing the Fermi functions, which for low temperatures is a second order process and small compared to the intrin-

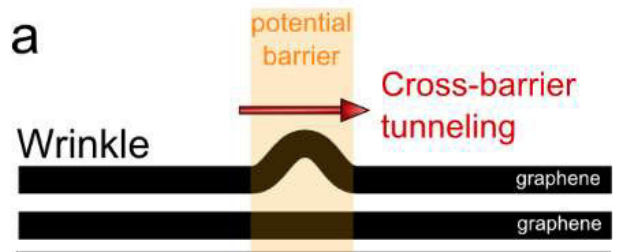

SiC+buffer layer

b

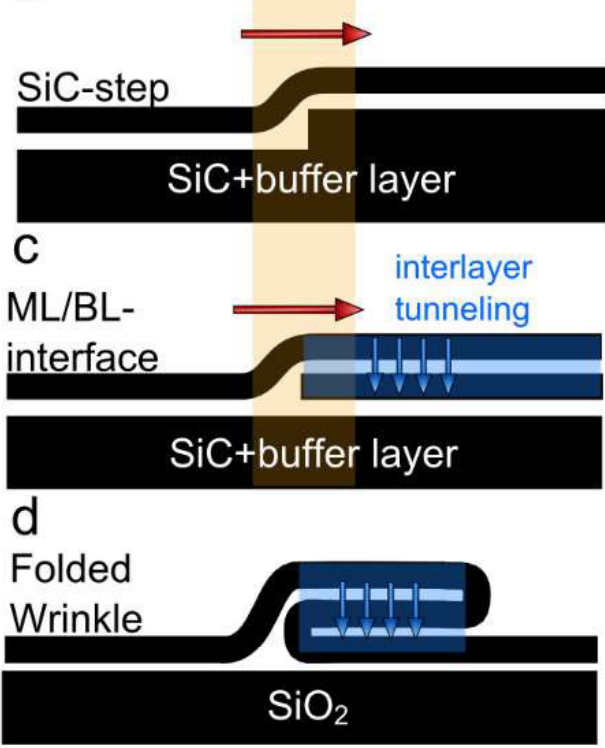

Figure 5.1: Tunneling across structural defects. (a) Bilayer wrinkle, (b) SiC-step, and (c) ML/BL-interface and (d) folded trilayer wrinkle (CVD-graphene on $\mathrm{SiO}_{2}$ ). The detachment from the substrate and buffer layer is inducing a change in charge carrier concentration. This creates a potential barrier through which can be tunneled. For the ML/BL-interface and the folded trilayer wrinkle additional interlayer tunneling is present that dominates in both cases the defect resistance. sic defect resistance of the junction $[183,188]$. This is supported by theoretical studies by Nilsson et al. who found no change in interlayer resistance in bilayer and multilayer graphene with temperature [131]. Similarly to temperature-independence, the presence of a magnetic field does not change the defect resistance, since it barely changes the k-vector components due to the small spatial extent of the defects. This has been calculated in 
subsection 3.4.5.10 of chapter 3 for a potential barrier model. Thus, quantum tunneling is a good candidate to explain the scattering at wrinkles and SiC-steps where tunneling/resonant scattering can occur along the graphene sheet. The electrons need to cross the potential barrier induced by the defect as shown in Figure 5.1a,b.

For the ML/BL-interface we introduced interlayer tunneling as a mechanism to explain the observed offset into the bilayer in section 3.2 of chapter 3 . This model sketched in Figure 5.1c would also be independent of B, T (See section 3.4). Consequently, the results of both studies from section 3.2 and 3.4 agree with this approach that treats the conduction of the two layers separately only coupled by the interlayer resistance. In Figure 5.1d we additionally sketch the defect resistance for a folded trilayer-like wrinkle which we measured for CVD-graphene on $\mathrm{SiO}_{2}$ in section 3.3. Here, the theoretical calculations by Zhu et al. already made interlayer tunneling responsible for the increased defect resistance [220]. Concerning our own work, we found the high value as well as the evidence for the temperature independence of the wrinkle resistance as indicators for this scattering mechanism. In a tight-binding model for bilayer graphene (see section 1.1 for monolayer graphene) the interlayer coupling is introduced via additional hopping parameters between different layers [151] leading to a different band structure compared to monolayer graphene. Thus, in this picture, a wave function mismatch for the transition from monolayer and bilayer leads to a finite reflectivity of electrons as it holds for quantum tunneling. This approach which had been suggested in Ref. [80] and which we introduced in section 4.1 to describe the scattering at ML/BL-junctions is sufficient to induce a large local resistance. Calculating the extended potential drop observed in section 3.2, however, remains much more feasible in the effective interlayer coupling model in section 3.2.7.6 and in section 3.3.3.3.

Ultimately, the identification and characterization of the scattering behavior might help to avoid defects of high resistance in sample growth and possibly harvest the different properties of defects compared to pristine sample regions in new device concepts.

\subsubsection{Comparison to defects in other systems}

The focus of this thesis and in other research groups in recent years has been local defects in graphene. However, other systems have been studied as well in the past by STP, KPFM, 4-point probe STM and transport experiments. Prominent systems are the surface state of $\mathrm{Si}(111)-7 \times 7[85,112,120]$ as well as the $\operatorname{Si}(111)-(\sqrt{3} \times$ $\sqrt{3})$-Ag surface $[71,112,122]$. Recently, bismuth selenide, a member of the emerging material class of topological insulators, has been studied by STP [11].

In Figure 5.2 we show the defect resistance $\rho_{\text {Defect }}$ along with the respective sheet conductivity $\sigma$ for the different systems. It shows that both quantities can vary over 
several orders of magnitude reflecting the different scattering mechanisms involved. We will try to make a few general statements from this collection of data.

- First, multiple steps show in all cases a higher resistivity than a monoatomic step. This follows roughly linear with increasing step height. ${ }^{27}$

- Second, for $\mathrm{Si}(111)-(\sqrt{3} \times \sqrt{3})-\mathrm{Ag}$ and graphene domain/grain boundaries one observes an even higher defect resistance then for the respective step resistance. This is however crucially depending on e.g. the specific misalignment between grains and the quality of the connection as argued in subsection 5.1.1 explaining the large spread of this defect resistance for graphene in Figure 5.2.

- We want to highlight that defects of graphene, in particular on SiC, show the lowest values of all defect resistances so far. With improved growth methods $[47,97]$ the presence of bilayer regions can be avoided leaving (monoatomic) SiC-substrate steps the only defect type present in this system.

- Moreover, the defect resistance $\rho_{\text {Defect }}$ seems to decrease for increasing conductivity $\sigma$. Whether this correlation is accidental or not is difficult to determine due to the huge differences in the origin of the conducting surface in each system and different mechanisms for the transmission $T$ at the defect. In general the conductivity is influenced by both the charge carrier concentration and the mobility $\sigma=e n \mu$. A possible explanation is a different scaling with charge carrier density $n$ : recalling the Landauer formula in Eq. (1.19) the defect resistance scales $\rho_{\text {Defect }}^{-1} \propto k_{F}$. Indeed, Tsen et al. found an increase of a grain boundary defect resistance with decreasing $n$ (consequently also decreasing $k_{F}$ ). Combining this with the conductivity from the Boltzmann-equation in Eq. (1.14) and the relation for a simple 2D electron gas $n=k_{F}^{2} / 2 \pi$ leads to $\rho_{\text {Defect }} \propto \sigma^{-1 / 2}$. Thus, a change in $n$ could explain a decrease of 2 orders of magnitude in $\rho_{\text {Defect }}$ for the 4 orders of $\sigma$ shown in Figure 5.2. This however assumes a $2 \mathrm{D}$ electron gas dispersion which is not always the case here, monolayer graphene being the prominent example. Besides the different dependences on $n$ the correlation between $\rho_{\text {Defect }}$ and $\sigma$ can stem from the fact that only at high conductivity of the sheets small defect resistances such as e.g. SiC-substrate steps become distinguishable from the linear background. This is nicely demonstrated in Figure 3.25a and Figure 3.27d where the contribution of the lo-

${ }^{27} \mathrm{Ji}$ et al. observed for epitaxial graphene a linear increase in defect resistance for SiC-substrate steps ranging from $0.5 \mathrm{~nm}-1.5 \mathrm{~nm}$ [80]. Bauer et al. found in $\mathrm{Bi}_{2} \mathrm{Se}_{3}$ for a 1 (3) quasilayer step a resistance of $9.1 \Omega \mu m(25 \Omega \mu m)$ [11]. Homoth et al. found for Si(111)- $(\sqrt{3} \times \sqrt{3})-A g$ a defect resistance of $313 \Omega \mu m(1429 \Omega \mu m)$ for a monoatomic step (multiple steps, not specified) [71]. 
calized defects is vanishing in this case by increasing the sheet resistance $^{28}$ with the external magnetic field. Therefore, small defect resistances, if present, are more difficult or impossible to detect at lower sheet conductivity which can be seen as a bias to the evaluation of local voltage drops in general.

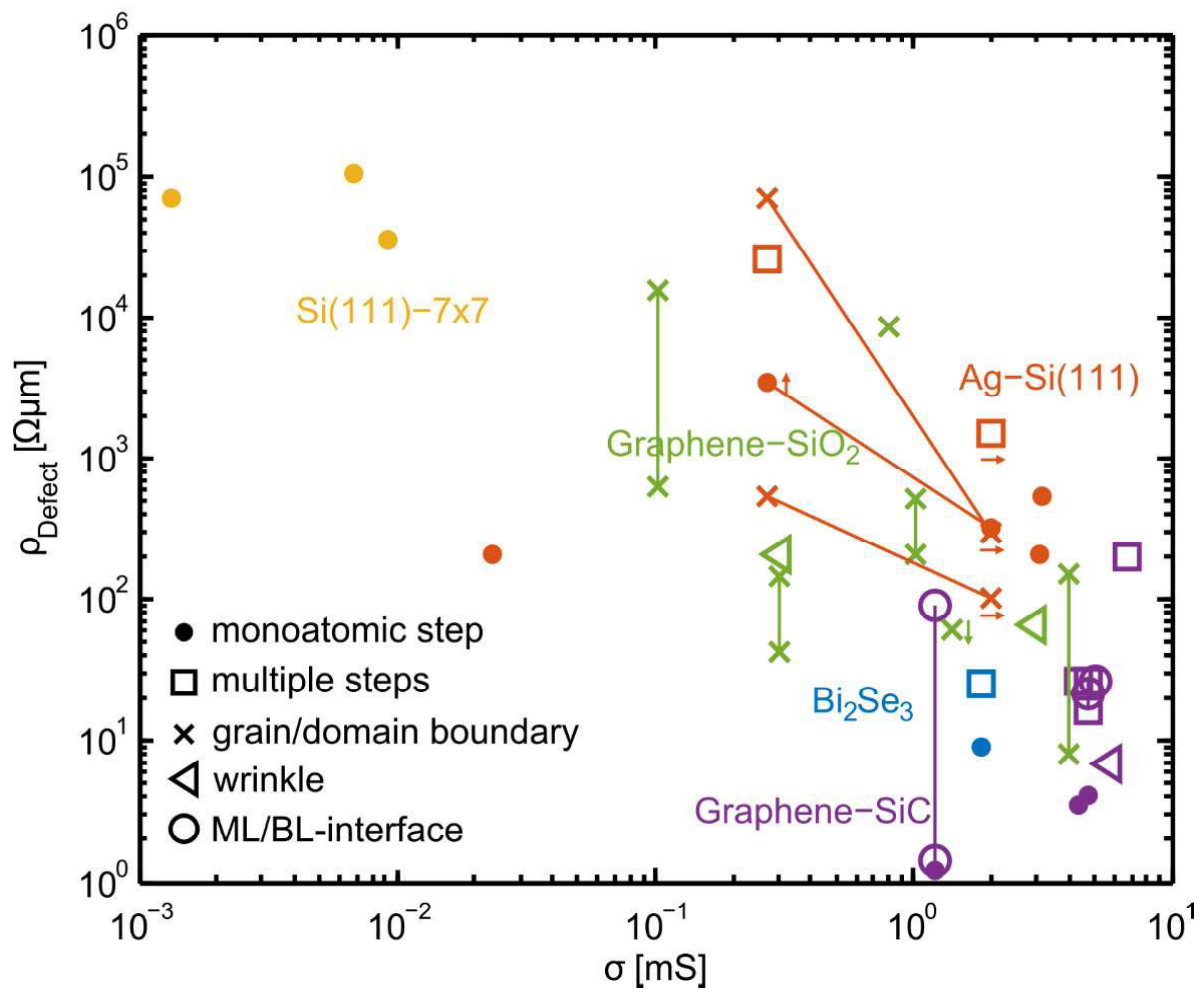

Figure 5.2: Overview for the resistance of local defects in different systems. Resistance of different defects $\rho_{\text {Defect }}$ over the sheet conductivity $\sigma$ of the respective system. Color-coded are different sample systems: $\mathrm{Si}(111)-7 \times 7$ (yellow), Graphene on $\mathrm{SiO}_{2}$ (green), Si(111)- $(\sqrt{3} \times \sqrt{3})-\mathrm{Ag}$ (red), Bismuth Selenide (blue) and Graphene on SiC (purple). Different symbols indicate different types of defects as described in the legend. For the sake of simplicity errorbars have been omitted. In case more than one value is given, lines connect the minimum and maximum value (For Si(111)- $(\sqrt{3} \times \sqrt{3})$-Ag: data has been evaluated for different current direction). Moreover, in case only a lower/higher bound was given for $\rho_{\text {Defect }} / \sigma$, the value is marked with an arrow pointing towards the possible range of values. Data was taken from following references: $\mathrm{Si}(111)-7 \times 7$ : Just et al.[85], Martins et al.[120] and Lüpke et al.[112]. Graphene on $\mathrm{SiO}_{2}$ : Clark et al.[30], Grosse et al.[61], Huang et al.[76],Tsen et al.[181] (p-doped case only), Yu et al.[210], Willke et al.[203]. Si(111)$(\sqrt{3} \times \sqrt{3})-\mathrm{Ag})$ : Homoth et al.[71], Matsuda et al.[122], Lüpke et al.[112]. $\mathrm{Bi}_{2} \mathrm{Se}_{3}$ : Bauer et al.[11]. Graphene on SiC: Willke et al.[202], Ji et al.[80], Clark et al.[30] and Ciuk et al.[28]. ${ }^{28}$ As argued in section 3.4 the sheet resistance is not increased intrinsically, but only the effective
scattering time of an electron in the sheet and thus the total resistance effectively scales with $B$. 
Explanations for backscattering at localized defects in the other sample systems have been manifold. This includes simple potential barriers at step edges for massless Dirac particles [92], allowed access to certain spin backscattering channels [127] as well as possible scattering into bulk states [11] $\left(\mathrm{Bi}_{2} \mathrm{Se}_{3}\right)$. For the monoatomic steps for $\mathrm{Si}(111)-7 \times 7$ Martins et al. found a strong decrease in a transmitting state at the Fermi level using DFT [120]. For monoatomic steps of Si(111)- $(\sqrt{3} \times$ $\sqrt{3})$-Ag Kobayashi developed an elaborate model based on the tight-binding method, the Landauer formalism as well as the transmission of Bloch waves. He found that the resistance of the step arises from the difference of Bloch wave numbers before and after the step as well as a discontinuity in their periodic part [91].

\subsection{Ion implantation of single dopants}

In chapter 4 we discussed our work on ion implantation in graphene. Besides introducing the technique and analyzing the structural and transport properties of substitutional atoms in graphene, especially the proof of charge transfer to induce both $\mathrm{p}$ - and $\mathrm{n}$-doping is of particular importance for application. A quantitative measure reflecting the ability of doping by atomic substitution is the charge transfer per atom. This number quantifies how much charge a nitrogen atom for instance adds to the graphene lattice and how much is found as localized charge at the doping atom and in its vicinity. Since both the atomic concentration of dopants as well as the position of the Fermi level is needed, this quantity has been experimentally obtained mostly by STM/STS-measurements. The charge carrier concentration can then be deduced for monolayer graphene via $n=E_{D}^{2} / \pi\left(\hbar v_{F}\right)^{2}$ [83]. Table 5.3 compares the different values found in literature for both nitrogen and boron doping. Our results agree with values obtained for other systems and with other methods. Deviations in the presented studies can be explained with a lack in accuracy of doping atom concentration plus errors in the evaluation of the position of the Dirac point $E_{D}$. Additionally, possible doping by residual defect concentrations other than graphitic substitution also differs in all studies (For instance pyridinic configuration for nitrogen which is actually leading to p-doping [178]). Our study finds the lowest charge transfer for nitrogen implantation, which however still agrees within the errorbars with values obtained by Zhao et al. [216]. For both experimental studies the charge transfer of boron is higher than that for the respective nitrogen case. In contrast boron atoms show a smaller spatial extent of the localized states in section 4.3 and in Ref. [217]. However, both values for nitrogen and boron show that roughly half of the electrons/holes are transferred to the graphene lattice.

The crucial question for application is how much the additional presence of doping atoms will alter the resistance and the mobility of the graphene sheet. A key result 
of chapter 4.3 is that the highest influence on transport stems from the additionally created lattice defects and not the doping atoms. For lattice defects, it has however been shown that they can be healed by annealing at high temperatures [88]. Considering nitrogen doping can give us a rough estimate on how much the mobility will be diminished. Here, we did not observe lattice defects in contrast to boron and carbon implanted samples. Taking the mobility data from Figure $4.11 \mathrm{~b}$ in the high temperature limit $(28 \mathrm{~K})$ where weak localization is not dominating transport anymore, the average resistance of the nitrogen-doped samples and the pristine samples is $\approx 1500 \Omega$ and $\approx 500 \Omega$, respectively. This leads to mobilities of $\approx 200$ $\mathrm{cm}^{2} /$ Vs and $\approx 1000 \mathrm{~cm}^{2} /$ Vs. Thus, the mobility decreases by a factor of 5 . The influence of the dopant atoms is consequently in an intermediate regime where it is not negligible, but also not completely destroying graphene's transport properties. Whether this is still suitable for application depends crucially on the requirements. For example, such a high implantation dose of $\approx 1 \%$ used in our experiment might not be needed for tuning the Fermi-level in the desired range.

\begin{tabular}{|c|c|c|c|c|}
\hline Publication & $\begin{array}{l}\text { Doping } \\
\text { type }\end{array}$ & $\begin{array}{c}\text { Charge transfer } \\
\text { [e/atom }]\end{array}$ & System & Method \\
\hline Zhao et al. & nitrogen & $0.42 \pm 0.07$ & Graphene on copper foil & STM/STS \\
\hline Joucken et al. & nitrogen & 0.8 & Graphene on $\mathrm{SiC}(000-1)$ & STM/STS \\
\hline Usachov et al. & nitrogen & 0.5 & $\begin{array}{l}\text { Graphene /Au /Ni(111) } \\
\text { M(110) }\end{array}$ & ARPES/XPS \\
\hline Willke et al. & nitrogen & $0.35 \pm 0.11$ & Graphene on $\mathrm{SiC}(0001)$ & STM/STS \\
\hline $\begin{array}{l}\text { Zhao et al. (The- } \\
\text { ory) }\end{array}$ & nitrogen & 0.6 & Graphene (Freestanding) & DFT \\
\hline $\begin{array}{l}\text { Joucken et al. } \\
\text { (Theory) }\end{array}$ & nitrogen & 0.55 & Graphene (Freestanding) & DFT \\
\hline Zhao et al. & boron & 0.5 & Graphene on copper foil & STM/STS \\
\hline Willke et al. & boron & $0.63 \pm 0.25$ & Graphene on SiC(0001) & STM/STS \\
\hline $\begin{array}{l}\text { Zhao et al. (The- } \\
\text { ory) }\end{array}$ & boron & 0.55 & Graphene on copper & DFT \\
\hline
\end{tabular}

Table 5.3. Charge transfer per dopant atom obtained for different systems and different techniques. Data taken from Ref. $[83,184,216,217]$ as well as chapter 4.3. 


\subsection{Outlook}

In this section we want to give an outlook of possible future experiments with the new methods and experimental preparation techniques developed in the course of this thesis.

Concerning the implantation of foreign atoms, the work shown in chapter 4 paves the way for subsequent studies. For the implantation of boron and nitrogen, besides the above mentioned possibility of reaching higher mobilities with higher post-implantation annealing temperature, it might additionally be of interest how far the system can be doped before losing its graphene properties. For doping with low concentrations of nitrogen atoms the change in charge carrier concentration was found to increase linearly [84]. Therefore, experiments using higher substitutional atom concentration are certainly interesting to test for a possible saturation or for a change in graphene's electronic properties. Moreover, due to the high flexibility of the method of ion implantation, other atomic species can be used. Aside from similar doping atoms such as aluminum, silicon and phosphorus more elaborate elements such as transition metals atoms can be used. This could not only create an interesting model system for different transport effects such as e.g. Kondo physics, which can then both be nicely addressed by STM and transport experiments, but could also combine magnetism with graphene, as recently realized using atomic hydrogen adatoms [58].

Additionally, weak localization at low temperatures observed for the present ionimplanted graphene samples can be additionally investigated on a local scale by STP. Here, the magnetotransport STP setup could determine on which scale weak localization acts in a sample or in other words how localized it really is.

Ultimately, STP might allow to access the voltage drop and thus the scattering properties around a single point defect, e.g. a nitrogen atom in graphene. This is summarized in the question 'How much resistance is added by a single nitrogen atom'? That it might be possible to answer has been shown in the Master's thesis of Thomas Kotzott [96]. Here, we estimated the influence of a defect atom on the potential drop observed by STP from resistor network simulations. Figure 5.3a shows the topography of a possible candidate system, an isolated substitutional nitrogen atom in SiC-graphene.

First, from the defect concentration and the subsequent increase in resistance (Figure 4.11a) both obtained in chapter 4.3 we can estimate the impact of a single nitrogen atom. A simple but useful description within a resistor network model as shown in Figure 5.3b describes the foreign atom with two parameters, a higher defect resistance $R_{\text {def }}$ and a radius of influence $\sigma$, in which the resistance differs from the average graphene resistance $R_{0}$. For the values used in this example (Figure $5.3 \mathrm{c}, R_{\text {def }}=1500 \Omega / \sigma=2.0 \mathrm{~nm}$ ) large scale simulations yield the same 
sheet resistance as observed in Figure 4.11a which we used as a boundary condition. As a result of the simulations we obtain the current distribution (Figure 5.3d) and the potential map (Figure 5.3e) with cross sections shown in Figure 5.3g. While the influence of the defect is barely seen, it becomes clearer with a subtraction of the linear background shown in Figure 5.3f/h. As can be seen in Figure 5.3h across the center of the defect the total change in potential is $\approx 40 \mu \mathrm{V}(j=13.3 \mathrm{~A} / \mathrm{m})$. This is still higher than the theoretical standard deviation of STP at room temperature $2 \mu \mathrm{V}[41]$ and therefore we should be able to detect the voltage signal for this configuration. However, the total amplitude also depends on the parameter set $\left(R_{\mathrm{def}} /\right.$ $\sigma$ ) which in a different configuration can lower the observed voltage drop. The signal-to-noise-ratio can be raised by applying a higher cross voltage. Here, the simulations however were already calculated with an average slope of $20 \mu \mathrm{V} / \mathrm{nm}$ which is one of the highest we measured up to now in graphene. At these high electric fields heat dissipation begins to limit the measurement time significantly due to additional helium boil off. This nevertheless could be limited by smaller and higher conducting pristine graphene samples.

This model is very simple and neglects for example any weak localization effects studied in section 4.3. Nevertheless, the resistor network model gives us a rough estimation on how large of a voltage drop can be expected for a single substitutional defect. These classical models worked well up to now even on an atomic length scale as demonstrated by the ML/BL-interface in section 3.2. Nevertheless, we like to note that this not necessarily yields the correct shape of the potential around the single defect as shown in Figure 5.3f. A quantum mechanical description of such a single impurity problem shows additional Friedel-like oscillations around the defect [222]. These however, have also been predicted at step edges [27] and have not observed so far. Either way, the experimental realization though challenging - would give new insights into truly atomic-scale transport. 
a

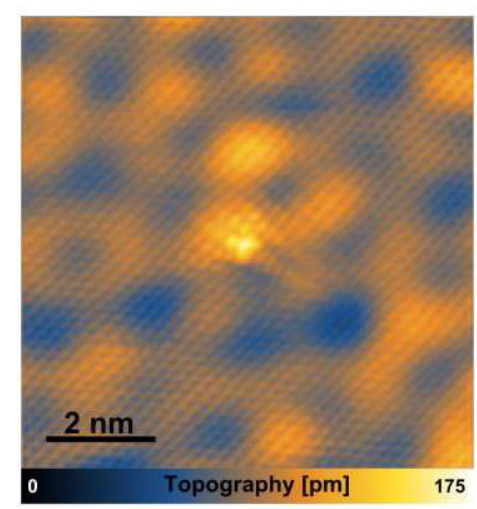

b


$f$

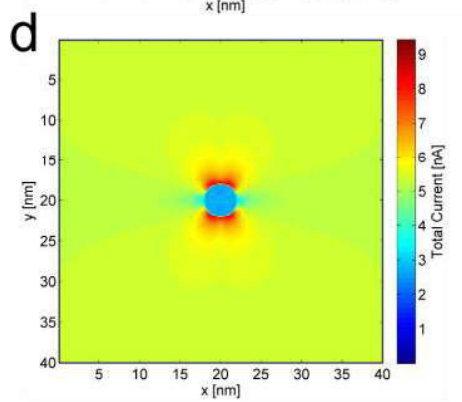

9

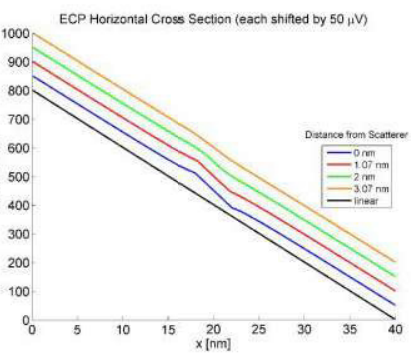

$\mathrm{h}$

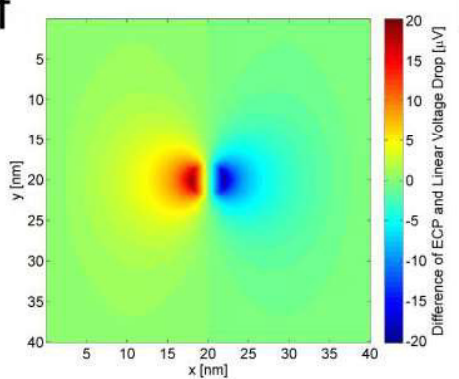

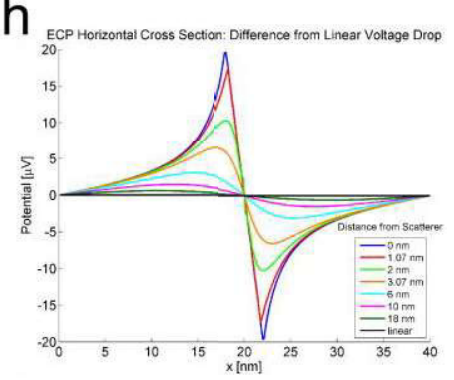

Figure 5.3: Local voltage drop around a single dopant atom. (a) Topography of an isolated substitutional implanted nitrogen atom in graphene on $\mathrm{SiC}\left(\mathrm{V}_{\text {Bias }}=-0.7 \mathrm{~V} / \mathrm{I}_{\mathrm{T}}=100\right.$ pA). (b) Resistor network with hard sphere model with defect resistance $R_{\text {def }}$ and radius $\sigma$. The knot of the defect is colored in bright red, all other knots within the radius influenced by the defect are blue. (c) Resistance map used in the simulations showing the resistor values for the resistors connecting the knots of the matrix. $\left(R_{\text {def }}=1500 \Omega / \sigma=2.0 \mathrm{~nm}\right)(\mathrm{d})$ sum of the absolute values of incoming and outgoing currents into every knot. (e) Electrochemical potential as it would be measured in an STP transport map (current density $\mathrm{j}=$ $13.3 \mathrm{~A} / \mathrm{m}$ ). (f) Electrochemical potential with subtracted linear slope of the external field. (g) Horizontal sections through (e) for different positions at the scatterer. Each line is shifted by $50 \mu \mathrm{V}$ for better readability. (h) Horizontal sections through (g) for different positions at the scatterer. (b-h: taken from Ref. [96].) 



\section{Bibliography}

[1] Elihu Abrahams, PW Anderson, PA Lee, and TV Ramakrishnan. Quasiparticle lifetime in disordered two-dimensional metals. Physical Review B, 24(12):6783, 1981.

[2] EH Åhlgren, J Kotakoski, and AV Krasheninnikov. Atomistic simulations of the implantation of low-energy boron and nitrogen ions into graphene. Physical Review B, 83(11):115424, 2011.

[3] Deji Akinwande, Nicholas Petrone, and James Hone. Two-dimensional flexible nanoelectronics. Nature communications, 5, 2014.

[4] SS Bae, N Prokopuk, NJ Quitoriano, SM Adams, and R Ragan. Characterizing defects and transport in si nanowire devices using kelvin probe force microscopy. Nanotechnology, 23(40):405706, 2012.

[5] Mario Norberto Baibich, Jean Marc Broto, Albert Fert, F Nguyen Van Dau, Frédéric Petroff, P Etienne, G Creuzet, A Friederich, and J Chazelas. Giant magnetoresistance of $(001) \mathrm{fe} /(001) \mathrm{cr}$ magnetic superlattices. Physical review letters, 61(21):2472, 1988.

[6] Richard Balog, Bjarke Jørgensen, Justin Wells, Erik Lægsgaard, Philip Hofmann, Flemming Besenbacher, and Liv Hornekær. Atomic hydrogen adsorbate structures on graphene. Journal of the American Chemical Society, 131(25):8744-8745, 2009.

[7] DA Bandurin, I Torre, R Krishna Kumar, M Ben Shalom, A Tomadin, A Principi, GH Auton, E Khestanova, KS Novoselov, IV Grigorieva, et al. Negative local resistance caused by viscous electron backflow in graphene. Science, 351(6277):1055-1058, 2016.

[8] U Bangert, W Pierce, DM Kepaptsoglou, Q Ramasse, R Zan, MH Gass, JA Van den Berg, CB Boothroyd, J Amani, and H Hofsäss. Ion implantation of graphene - toward ic compatible technologies. Nano letters, 13(10):49024907, 2013.

[9] A Bannani, CA Bobisch, and R Möller. Local potentiometry using a multiprobe scanning tunneling microscope. Review of scientific instruments, 79(8):083704, 2008.

[10] J. Bardeen. Tunneling from a many particle point of view. Physical Review Letters, 6 (2):57-59, 1961.

[11] Sebastian Bauer and Christian A Bobisch. Nanoscale electron transport at the surface of a topological insulator. Nature communications, 7, 2016.

[12] Claire Berger, Zhimin Song, Tianbo Li, Xuebin Li, Asmerom Y Ogbazghi, Rui Feng, Zhenting Dai, Alexei N Marchenkov, Edward H Conrad, Phillip N First, 
et al. Ultrathin epitaxial graphite: $2 \mathrm{~d}$ electron gas properties and a route toward graphene-based nanoelectronics. The Journal of Physical Chemistry B, 108(52):19912-19916, 2004.

[13] Claire Berger, Zhimin Song, Xuebin Li, Xiaosong Wu, Nate Brown, Cécile Naud, Didier Mayou, Tianbo Li, Joanna Hass, Alexei N Marchenkov, et al. Electronic confinement and coherence in patterned epitaxial graphene. Science, 312(5777):1191-1196, 2006.

[14] DE Beutler and N Giordano. Localization and electron-electron interaction effects in thin bi wires and films. Physical Review B, 38(1):8, 1988.

[15] Kirk H Bevan. Atomistic linear response voltage drop calculations for quantum transport in materials: the high conductance regime. Journal of Applied Physics, 114(10):103705, 2013.

[16] Kirk H Bevan. A first principles scanning tunneling potentiometry study of an opaque graphene grain boundary in the ballistic transport regime. Nanotechnology, 25(41):415701, 2014.

[17] Gerd Binnig, Calvin F Quate, and Ch Gerber. Atomic force microscope. Physical review letters, 56(9):930, 1986.

[18] Gerd Binnig, Heinrich Rohrer, Ch Gerber, and El Weibel. Surface studies by scanning tunneling microscopy. Physical review letters, 49(1):57, 1982.

[19] D. Bonnell. Scanning Tunneling Microscopy and Spectroscopy. Theory, Techniques, and Applications. VCH, New York, 2001.

[20] Aaron Bostwick, Taisuke Ohta, Thomas Seyller, Karsten Horn, and Eli Rotenberg. Quasiparticle dynamics in graphene. Nature Physics, 3(1):36-40, 2007.

[21] BG Briner, RM Feenstra, TP Chin, and JM Woodall. Local transport properties of thin bismuth films studied by scanning tunneling potentiometry. Physical Review B, 54(8):R5283, 1996.

[22] Sudip Chakravarty and Albert Schmid. Weak localization: The quasiclassical theory of electrons in a random potential. Physics Reports, 140(4):193-236, 1986.

[23] J-C Charlier, J-P Michenaud, Xavier Gonze, and J-P Vigneron. Tight-binding model for the electronic properties of simple hexagonal graphite. Physical Review B, 44(24):13237, 1991.

[24] J. Chen. Introduction to Scanning Tunneling Microscopy. Oxford University Press, 1993.

[25] Jian-Hao Chen, Chaun Jang, Shudong Xiao, Masa Ishigami, and Michael S Fuhrer. Intrinsic and extrinsic performance limits of graphene devices on sio2. Nature nanotechnology, 3(4):206-209, 2008.

[26] Wei Chen, Shi Chen, Dong Chen Qi, Xing Yu Gao, and Andrew Thye Shen Wee. Surface transfer p-type doping of epitaxial graphene. Journal of the American Chemical Society, 129(34):10418-10422, 2007. 
[27] CS Chu and RS Sorbello. Phase-sensitive scanning tunneling potentiometry and the local transport field in mesoscopic systems. Physical Review B, 42(8):4928, 1990.

[28] Tymoteusz Ciuk, Semih Cakmakyapan, Ekmel Ozbay, Piotr Caban, Kacper Grodecki, Aleksandra Krajewska, Iwona Pasternak, Jan Szmidt, and Wlodek Strupinski. Step-edge-induced resistance anisotropy in quasi-free-standing bilayer chemical vapor deposition graphene on sic. Journal of Applied Physics, 116(12):123708, 2014.

[29] Kendal W Clark, X-G Zhang, Gong Gu, Jewook Park, Guowei He, RM Feenstra, and An-Ping Li. Energy gap induced by friedel oscillations manifested as transport asymmetry at monolayer-bilayer graphene boundaries. Physical Review X, 4(1):011021, 2014.

[30] Kendal W Clark, X-G Zhang, Ivan V Vlassiouk, Guowei He, Randall M Feenstra, and An-Ping Li. Spatially resolved mapping of electrical conductivity across individual domain (grain) boundaries in graphene. ACS nano, 7(9):7956-7966, 2013.

[31] Supriyo Datta. Electronic transport in mesoscopic systems. Cambridge university press, 1997.

[32] John H Davies. The physics of low-dimensional semiconductors: an introduction. Cambridge university press, 1997.

[33] R. S. Deacon, K.-C. Chuang, R. J. Nicholas, K. S. Novoselov, and A. K. Geim. Cyclotron resonance study of the electron and hole velocity in graphene monolayers. Phys. Rev. B, 76:081406, Aug 2007.

[34] Cory R Dean, Andrea F Young, Inanc Meric, Chris Lee, Lei Wang, S Sorgenfrei, K Watanabe, T Taniguchi, P Kim, KL Shepard, et al. Boron nitride substrates for high-quality graphene electronics. Nature nanotechnology, 5(10):722-726, 2010.

[35] Michael P Delmo, Shinpei Yamamoto, Shinya Kasai, Teruo Ono, and Kensuke Kobayashi. Large positive magnetoresistive effect in silicon induced by the space-charge effect. Nature, 457(7233):1112-1115, 2009.

[36] Dehui Deng, Xiulian Pan, Liang Yu, Yi Cui, Yeping Jiang, Jing Qi, Wei-Xue $\mathrm{Li}$, Qiang Fu, Xucun Ma, Qikun Xue, et al. Toward n-doped graphene via solvothermal synthesis. Chemistry of Materials, 23(5):1188-1193, 2011.

[37] Quantum Design. Ppsm system features. Rev.A0.

[38] Mildred S Dresselhaus and G Dresselhaus. Intercalation compounds of graphite. Advances in physics, 51(1):1-186, 2002.

[39] Paul Drude. Zur elektronentheorie der metalle. Annalen der Physik, 306(3):566-613, 1900.

[40] Paul Drude. Zur ionentheorie der metalle. Physikalische Zeitschrift, 1:161165, 1900.

[41] T Druga, M Wenderoth, J Homoth, MA Schneider, and RG Ulbrich. A versatile high resolution scanning tunneling potentiometry implementation. Review of Scientific Instruments, 81(8):083704, 2010. 
[42] Thomas Druga. Graphen auf Siliziumkarbid: elektronische Eigenschaften und Ladungstransport. PhD thesis, Georg-August-Universität Göttingen, 2014

[43] Thomas Druga, Martin Wenderoth, Felix Lüpke, and Rainer G Ulbrich. Graphene-metal contact resistivity on semi-insulating 6h-sic (0001) measured with kelvin probe force microscopy. Applied Physics Letters, 103(5):051601, 2013.

[44] Dinh Loc Duong, Gang Hee Han, Seung Mi Lee, Fethullah Gunes, Eun Sung Kim, Sung Tae Kim, Heetae Kim, Quang Huy Ta, Kang Pyo So, Seok Jun Yoon, et al. Probing graphene grain boundaries with optical microscopy. Nature, 490(7419):235-239, 2012.

[45] W Eckstein, R Dohmen, A Mutzke, and R Schneider. Sdtrimsp: Ein montecarlo code zur berechnung von stossereignissen in ungeordneten targets= sdtrimsp: A monte-carlo code for calculating collision phenomena in randomized targets. 2007.

[46] Konstantin $\vee$ Emtsev, Aaron Bostwick, Karsten Horn, Johannes Jobst, Gary L Kellogg, Lothar Ley, Jessica L McChesney, Taisuke Ohta, Sergey A Reshanov, Jonas Röhrl, et al. Towards wafer-size graphene layers by atmospheric pressure graphitization of silicon carbide. Nature materials, 8(3):203-207, 2009.

[47] Konstantin V Emtsev, Alexei A Zakharov, Camilla Coletti, Stiven Forti, and Ulrich Starke. Ambipolar doping in quasifree epitaxial graphene on sic (0001) controlled by ge intercalation. Physical Review B, 84(12):125423, 2011.

[48] KV Emtsev, F Speck, Th Seyller, L Ley, and John Douglas Riley. Interaction, growth, and ordering of epitaxial graphene on sic $\{0001\}$ surfaces: A comparative photoelectron spectroscopy study. Physical Review $B$, 77(15):155303, 2008.

[49] Klaus Engel. Raster-Thermospannungs-Mikroskopie der Interferenz von Elektronenwellen auf der Au(111)-Oberfläche. PhD thesis, Georg-AugustUniversität Göttingen, 2001.

[50] David Ferry and Stephen Marshall Goodnick. Transport in nanostructures. Number 6. Cambridge university press, 1997.

[51] T Filleter and R Bennewitz. Structural and frictional properties of graphene films on sic (0001) studied by atomic force microscopy. Physical Review B, 81(15):155412, 2010.

[52] I Forbeaux, J-M Themlin, and J-M Debever. Heteroepitaxial graphite on 6 hsic (0001): Interface formation through conduction-band electronic structure. Physical Review B, 58(24):16396, 1998.

[53] JK Garleff, M Wenderoth, K Sauthoff, RG Ulbrich, and M Rohlfing. 2× 1 reconstructed si (111) surface: Stm experiments versus ab initio calculations. Physical Review B, 70(24):245424, 2004.

[54] J Gebhardt, RJ Koch, W Zhao, O Höfert, K Gotterbarm, S Mammadov, C Papp, A Görling, H-P Steinrück, and Th Seyller. Growth and electronic 
structure of boron-doped graphene. Physical Review B, 87(15):155437, 2013.

[55] Andre K Geim and Irina V Grigorieva. Van der waals heterostructures. Nature, 499(7459):419-425, 2013.

[56] Andre K Geim and Konstantin S Novoselov. The rise of graphene. Nature materials, 6(3):183-191, 2007.

[57] F Giannazzo, I Deretzis, A La Magna, F Roccaforte, and Rositsa Yakimova. Electronic transport at monolayer-bilayer junctions in epitaxial graphene on sic. Physical Review B, 86(23):235422, 2012.

[58] Héctor González-Herrero, José M Gómez-Rodrguez, Pierre Mallet, Mohamed Moaied, Juan José Palacios, Carlos Salgado, Miguel M Ugeda, Jean-Yves Veuillen, Félix Yndurain, and Iván Brihuega. Atomic-scale control of graphene magnetism by using hydrogen atoms. Science, 352(6284):437441, 2016.

[59] Benjamin Grévin, Ivan Maggio-Aprile, Andreas Bentzen, L Ranno, A Llobet, and $\varnothing$ Fischer. Local electronic transport in la $0.7 \mathrm{sr} 0.3$ mno 3 thin films studied by scanning tunneling potentiometry. Physical Review $B$, 62(13):8596, 2000.

[60] Rudolf Gross and Achim Marx. Festkörperphysik. Walter de Gruyter GmbH \& Co KG, 2014.

[61] Kyle L Grosse, Vincent E Dorgan, David Estrada, Joshua D Wood, Ivan Vlassiouk, Gyula Eres, Joseph W Lyding, William P King, and Eric Pop. Direct observation of resistive heating at graphene wrinkles and grain boundaries. Applied Physics Letters, 105(14):143109, 2014.

[62] Peter Grünberg, R Schreiber, Y Pang, MB Brodsky, and H Sowers. Layered magnetic structures: evidence for antiferromagnetic coupling of fe layers across cr interlayers. Physical Review Letters, 57(19):2442, 1986.

[63] Masayuki Hamada and Yukio Hasegawa. Development of scanning tunneling potentiometry for semiconducting samples. Japanese Journal of Applied Physics, 51(12R):125202, 2012.

[64] R. J. Hamers. Atomic resolution surface spectroscopy with the scanning tunneling microscope. Annu. Rev. Phys. Chem., 40:531, 1989.

[65] Inga Karolin Hannstein. Self-organized formation of metal-carbon nanostructures by hyperthermal ion deposition. 2006.

[66] J Hass, WA De Heer, and EH Conrad. The growth and morphology of epitaxial multilayer graphene. Journal of Physics: Condensed Matter, 20(32):323202, 2008.

[67] AJ Heinrich, M Wenderoth, KJ Engel, TCG Reusch, K Sauthoff, RG Ulbrich, ER Weber, and K Uchida. Short-range ordering in al $x$ ga $1-x$ as grown with metal-organic vapor-phase epitaxy. Physical Review B, 59(15):10296, 1999.

[68] Charlotte Herbig, E Harriet Åhlgren, Wouter Jolie, Carsten Busse, Jani Kotakoski, Arkady $\vee$ Krasheninnikov, and Thomas Michely. Interfacial carbon 
nanoplatelet formation by ion irradiation of graphene on iridium (111). ACS nano, 8(12):12208-12218, 2014.

[69] H Hibino, S Mizuno, H Kageshima, M Nagase, and H Yamaguchi. Stacking domains of epitaxial few-layer graphene on sic (0001). Physical Review $B$, 80(8):085406, 2009.

[70] Bernd Hoefflinger. Itrs 2028-international roadmap of semiconductors. In CHIPS 2020 VOL. 2, pages 143-148. Springer, 2016.

[71] J Homoth, M Wenderoth, T Druga, L Winking, RG Ulbrich, CA Bobisch, B Weyers, A Bannani, E Zubkov, AM Bernhart, et al. Electronic transport on the nanoscale: ballistic transmission and ohm's law. Nano letters, 9(4):15881592, 2009.

[72] Jan Homoth. Das elektrochemische Potential auf der atomaren Skala: Untersuchung des Ladungstransports eines stromtragenden zweidimensionalen Elektronengases mit Hilfe der Raster-Tunnel-Mikroskopie. PhD thesis, Georg-August-Universität Göttingen, 2008.

[73] X Hong, S-H Cheng, C Herding, and J Zhu. Colossal negative magnetoresistance in dilute fluorinated graphene. Physical Review B, 83(8):085410, 2011.

[74] TW Hu, Fei Ma, DY Ma, D Yang, XT Liu, KW Xu, and Paul K Chu. Evidence of atomically resolved $6 \times 6$ buffer layer with long-range order and short-range disorder during formation of graphene on 6h-sic by thermal decomposition. Applied Physics Letters, 102(17):171910, 2013.

[75] Han Huang, Swee Liang Wong, Jiatao Sun, Wei Chen, and Andrew Thye Shen Wee. Trapping single polar molecules in sic nanomesh via outof-plane dipoles. ACS nano, 6(3):2774-2778, 2012.

[76] Pinshane $Y$ Huang, Carlos S Ruiz-Vargas, Arend M van der Zande, William S Whitney, Mark P Levendorf, Joshua W Kevek, Shivank Garg, Jonathan S Alden, Caleb J Hustedt, Ye Zhu, et al. Grains and grain boundaries in single-layer graphene atomic patchwork quilts. Nature, 469(7330):389-392, 2011.

[77] Siegfried Hunklinger. Festkörperphysik. Oldenbourg Verlag, 2009.

[78] Harald Ibach and Hans Lüth. Solid-state physics: an introduction to principles of material science. Advanced Texts in Physics, Springer-Verlag berlin Heidelberg New York, 2003.

[79] I Isenberg, BR Russell, and RF Greene. Improved method for measuring hall coefficients. Review of Scientific Instruments, 19(10):685-688, 1948.

[80] Shuai-Hua Ji, JB Hannon, RM Tromp, V Perebeinos, J Tersoff, and FM Ross. Atomic-scale transport in epitaxial graphene. Nature materials, 11(2):114-119, 2012.

[81] Johannes Jobst, Daniel Waldmann, Igor V Gornyi, Alexander D Mirlin, and Heiko B Weber. Electron-electron interaction in the magnetoresistance of graphene. Physical review letters, 108(10):106601, 2012. 
[82] Johannes Jobst, Daniel Waldmann, Florian Speck, Roland Hirner, Duncan K Maude, Thomas Seyller, and Heiko B Weber. Quantum oscillations and quantum hall effect in epitaxial graphene. Physical Review B, 81(19):195434, 2010.

[83] Frédéric Joucken, Yann Tison, Jérôme Lagoute, Jacques Dumont, Damien Cabosart, Bing Zheng, Vincent Repain, Cyril Chacon, Yann Girard, Andrés Rafael Botello-Méndez, et al. Localized state and charge transfer in nitrogen-doped graphene. Physical Review B, 85(16):161408, 2012.

[84] Frederic Joucken, Yann Tison, Patrick Le Fevre, Antonio Tejeda, Amina Taleb-Ibrahimi, Edward Conrad, Vincent Repain, Cyril Chacon, Amandine Bellec, Yann Girard, et al. Charge transfer and electronic doping in nitrogendoped graphene. Scientific reports, 5, 2015.

[85] Sven Just, Marcus Blab, Stefan Korte, Vasily Cherepanov, Helmut Soltner, and Bert Voigtländer. Surface and step conductivities on si (111) surfaces. Physical review letters, 115(6):066801, 2015.

[86] MI Katsnelson, KS Novoselov, and AK Geim. Chiral tunnelling and the klein paradox in graphene. Nature physics, 2(9):620-625, 2006.

[87] J Kautz, J Jobst, C Sorger, RM Tromp, HB Weber, and SJ van der Molen. Low-energy electron potentiometry: Contactless imaging of charge transport on the nanoscale. Scientific reports, 5, 2015.

[88] Ki-jeong Kim, Sena Yang, Youngchan Park, Myungjin Lee, BongSoo Kim, and Hangil Lee. Annealing effects after nitrogen ion casting on monolayer and multilayer graphene. The Journal of Physical Chemistry C, 117(5):21292134, 2013.

[89] Ferdinand Kisslinger, Christian Ott, Christian Heide, Erik Kampert, Benjamin Butz, Erdmann Spiecker, Sam Shallcross, and Heiko B Weber. Linear magnetoresistance in mosaic-like bilayer graphene. Nature Physics, 11(8):650653, 2015.

[90] Ferdinand Kisslinger, Christian Ott, and Heiko B Weber. On the origin of nonsaturating linear magnetoresistivity. arXiv preprint arXiv:1609.02418, 2016.

[91] Katsuyoshi Kobayashi. Electrical resistance across a step of the si (111)-ag surface. Surface science, 583(1):16-28, 2005.

[92] Katsuyoshi Kobayashi. Electron transmission through atomic steps of bi 2 se 3 and bi 2 te 3 surfaces. Physical Review B, 84(20):205424, 2011.

[93] Coskun Kocabas and Sefik Suzer. Probing voltage drop variations in graphene with photoelectron spectroscopy. Analytical chemistry, 85(8):41724177, 2013.

[94] Justin C Koepke, Joshua D Wood, David Estrada, Zhun-Yong Ong, Kevin T $\mathrm{He}$, Eric Pop, and Joseph W Lyding. Atomic-scale evidence for potential barriers and strong carrier scattering at graphene grain boundaries: a scanning tunneling microscopy study. ACS nano, 7(1):75-86, 2013. 
[95] Mikito Koshino, Takeshi Nakanishi, and Tsuneya Ando. Interface landau levels in graphene monolayer-bilayer junctions. Physical Review $B$, 82(20):205436, 2010.

[96] Thomas Kotzott. Magnetotransport in graphene on the atomic scale. Master's thesis, Georg-August-Universität Göttingen, 2015.

[97] Mattias Kruskopf, Davood Momeni Pakdehi, Klaus Pierz, Stefan Wundrack, Rainer Stosch, Thorsten Dziomba, Martin Götz, Jens Baringhaus, Johannes Aprojanz, Christoph Tegenkamp, Jakob Lidzba, Thomas Seyller, Frank Hohls, Franz J Ahlers, and Hans W Schumacher. Comeback of epitaxial graphene for electronics: large-area growth of bilayer-free graphene on sic. $2 D$ Materials, 3(4):041002, 2016.

[98] Sam Ladak, JM Ball, Daniel Moseley, G Eda, WR Branford, M Chhowalla, TD Anthopoulos, and LF Cohen. Observation of wrinkle induced potential drops in biased chemically derived graphene thin film networks. Carbon, 64:35-44, 2013.

[99] Rolf Landauer. Spatial variation of currents and fields due to localized scatterers in metallic conduction. IBM Journal of Research and Development, 1(3):223-231, 1957.

[100] P Lauffer, KV Emtsev, R Graupner, Th Seyller, L Ley, SA Reshanov, and HB Weber. Atomic and electronic structure of few-layer graphene on sic (0001) studied with scanning tunneling microscopy and spectroscopy. Physical Review B, 77(15):155426, 2008.

[101] Jae-Hyun Lee, Eun Kyung Lee, Won-Jae Joo, Yamujin Jang, Byung-Sung Kim, Jae Young Lim, Soon-Hyung Choi, Sung Joon Ahn, Joung Real Ahn, Min-Ho Park, et al. Wafer-scale growth of single-crystal monolayer graphene on reusable hydrogen-terminated germanium. Science, 344(6181):286-289, 2014.

[102] An-Ping Li, Kendal W Clark, X-G Zhang, and Arthur P Baddorf. Electron transport at the nanometer-scale spatially revealed by four-probe scanning tunneling microscopy. Advanced Functional Materials, 23(20):2509-2524, 2013.

[103] Xuesong Li, Weiwei Cai, Jinho An, Seyoung Kim, Junghyo Nah, Dongxing Yang, Richard Piner, Aruna Velamakanni, Inhwa Jung, Emanuel Tutuc, et al. Large-area synthesis of high-quality and uniform graphene films on copper foils. Science, 324(5932):1312-1314, 2009.

[104] Xuesong Li, Yanwu Zhu, Weiwei Cai, Mark Borysiak, Boyang Han, David Chen, Richard D Piner, Luigi Colombo, and Rodney S Ruoff. Transfer of large-area graphene films for high-performance transparent conductive electrodes. Nano letters, 9(12):4359-4363, 2009.

[105] Tomas Löfwander, Pablo San-Jose, and Elsa Prada. Quantum hall effect in graphene with twisted bilayer stripe defects. Physical Review B, 87(20):205429, 2013. 
[106] David C Look. Review of hall effect and magnetoresistance measurements in gaas materials and devices. Journal of The Electrochemical Society, 137(1):260-266, 1990.

[107] Emanuel Lörtscher. Wiring molecules into circuits. Nature nanotechnology, 8(6):381-384, 2013.

[108] Tony Low, Vasili Perebeinos, Jerry Tersoff, and Ph Avouris. Deformation and scattering in graphene over substrate steps. Physical review letters, 108(9):096601, 2012.

[109] Jiong Lu, Pei Shan Emmeline Yeo, Yi Zheng, Zhiyong Yang, Qiaoliang Bao, Chee Kwan Gan, and Kian Ping Loh. Using the graphene moiré pattern for the trapping of c60 and homoepitaxy of graphene. ACS nano, 6(1):944-950, 2011.

[110] BM Ludbrook, Giorgio Levy, Pascal Nigge, Marta Zonno, Michael Schneider, DJ Dvorak, CN Veenstra, Sergey Zhdanovich, Douglas Wong, Pinder Dosanjh, et al. Evidence for superconductivity in li-decorated monolayer graphene. Proceedings of the National Academy of Sciences, 112(38):1179511799, 2015.

[111] A Luican, Guohong Li, A Reina, J Kong, RR Nair, Konstantin S Novoselov, Andre K Geim, and EY Andrei. Single-layer behavior and its breakdown in twisted graphene layers. Physical review letters, 106(12):126802, 2011.

[112] F Lüpke, S Korte, V Cherepanov, and B Voigtländer. Scanning tunneling potentiometry implemented into a multi-tip setup by software. Review of scientific instruments, 86(12):123701, 2015.

[113] Ruitao Lv, Gugang Chen, Qing Li, Amber McCreary, Andrés Botello-Méndez, SV Morozov, Liangbo Liang, Xavier Declerck, Nestor Perea-López, David A Cullen, et al. Ultrasensitive gas detection of large-area boron-doped graphene. Proceedings of the National Academy of Sciences, 112(47):1452714532, 2015.

[114] Eric Yue Ma, Yong-Tao Cui, Kentaro Ueda, Shujie Tang, Kai Chen, Nobumichi Tamura, Phillip M Wu, Jun Fujioka, Yoshinori Tokura, and Zhi-Xun Shen. Mobile metallic domain walls in an all-in-all-out magnetic insulator. Science, 350(6260):538-541, 2015.

[115] Ather Mahmood, Cécile Naud, Clément Bouvier, Fanny Hiebel, Pierre Mallet, J-Y Veuillen, LP Lévy, Didier Chaussende, and Thierry Ouisse. Epitaxial graphene morphologies probed by weak (anti)-localization. Journal of Applied Physics, 113(8):083715, 2013.

[116] Petro Maksymovych, Simon J Kelly, and Jorge I Cerdá. Surface-state enhancement of tunneling thermopower on the ag (111) surface. ACS nano, 8(12):12110-12119, 2014.

[117] Pierre Mallet, Ivan Brihuega, Sangita Bose, Miguel Moreno Ugeda, José M Gomez-Rodriguez, Klaus Kern, and Jean-Yves Veuillen. Role of pseudospin in quasiparticle interferences in epitaxial graphene probed by high-resolution scanning tunneling microscopy. Physical Review B, 86(4):045444, 2012. 
[118] Pierre Mallet, François Varchon, Cécile Naud, Laurence Magaud, Claire Berger, and J-Y Veuillen. Electron states of mono-and bilayer graphene on sic probed by scanning-tunneling microscopy. Physical Review $B$, 76(4):041403, 2007.

[119] Jens Martin, N Akerman, G Ulbricht, T Lohmann, JH v Smet, K Von Klitzing, and Amir Yacoby. Observation of electron-hole puddles in graphene using a scanning single-electron transistor. Nature Physics, 4(2):144-148, 2008.

[120] Bruno VC Martins, Manuel Smeu, Lucian Livadaru, Hong Guo, and Robert A Wolkow. Conductivity of si (111)-(7×7): the role of a single atomic step. Physical review letters, 112(24):246802, 2014.

[121] K Matsubara, K Sugihara, and T Tsuzuku. Electrical resistance in the c direction of graphite. Physical Review B, 41(2):969, 1990.

[122] Iwao Matsuda, Masashi Ueno, Toru Hirahara, Rei Hobara, Harumo Morikawa, Canhua Liu, and Shuji Hasegawa. Electrical resistance of a monatomic step on a crystal surface. Physical review letters, 93(23):236801, 2004.

[123] Edward McCann, K Kechedzhi, Vladimir I Fal'ko, H Suzuura, T Ando, and BL Altshuler. Weak-localization magnetoresistance and valley symmetry in graphene. Physical Review Letters, 97(14):146805, 2006.

[124] Michael J McLennan, Yong Lee, and Supriyo Datta. Voltage drop in mesoscopic systems: A numerical study using a quantum kinetic equation. Physical Review B, 43(17):13846, 1991.

[125] P Muralt and DW Pohl. Scanning tunneling potentiometry. Applied Physics Letters, 48(8):514-516, 1986.

[126] Takeshi Nakanishi, Mikito Koshino, and Tsuneya Ando. Transmission through a boundary between monolayer and bilayer graphene. Physical Review $B$, 82(12):125428, 2010.

[127] Awadhesh Narayan, Ivan Rungger, Andrea Droghetti, and Stefano Sanvito. $\mathrm{Ab}$ initio transport across bismuth selenide surface barriers. Physical Review $B$, 90(20):205431, 2014.

[128] Alpha T. N'Diaye, Sebastian Bleikamp, Peter J. Feibelman, and Thomas Michely. Two-dimensional ir cluster lattice on a graphene moiré on ir(111). Phys. Rev. Lett., 97:215501, Nov 2006.

[129] AH Castro Neto, F Guinea, Nuno MR Peres, Kostya S Novoselov, and Andre K Geim. The electronic properties of graphene. Reviews of modern physics, 81(1):109, 2009.

[130] Johan Nilsson, AH Castro Neto, F Guinea, and NMR Peres. Transmission through a biased graphene bilayer barrier. Physical Review B, 76(16):165416, 2007.

[131] Johan Nilsson, AH Castro Neto, F Guinea, and NMR Peres. Electronic properties of bilayer and multilayer graphene. Physical Review B, 78(4):045405, 2008.

[132] Abraham Nitzan and Mark A Ratner. Electron transport in molecular wire junctions. Science, 300(5624):1384-1389, 2003. 
[133] AP Nizovtsev, S Ya Kilin, F Jelezko, T Gaebal, I Popa, A Gruber, and $\mathrm{J}$ Wrachtrup. A quantum computer based on nv centers in diamond: optically detected nutations of single electron and nuclear spins. Optics and spectroscopy, 99(2):233-244, 2005.

[134] Wataru Norimatsu and Michiko Kusunoki. Structural features of epitaxial graphene on sic0001 surfaces. Journal of Physics D: Applied Physics, 47(9):094017, 2014.

[135] Kostya S Novoselov, Andre K Geim, Sergei V Morozov, D Jiang, Y_Zhang, Sergey V Dubonos, Irina V Grigorieva, and Alexandr A Firsov. Electric field effect in atomically thin carbon films. science, 306(5696):666-669, 2004.

[136] KSA Novoselov, Andre K Geim, SVb Morozov, Da Jiang, Mlc Katsnelson, IVa Grigorieva, SVb Dubonos, and AAb Firsov. Two-dimensional gas of massless dirac fermions in graphene. nature, 438(7065):197-200, 2005.

[137] Taisuke Ohta, Aaron Bostwick, Jessica L McChesney, Thomas Seyller, Karsten Horn, and Eli Rotenberg. Interlayer interaction and electronic screening in multilayer graphene investigated with angle-resolved photoemission spectroscopy. Physical Review Letters, 98(20):206802, 2007.

[138] Taisuke Ohta, Aaron Bostwick, Thomas Seyller, Karsten Horn, and Eli Rotenberg. Controlling the electronic structure of bilayer graphene. Science, 313(5789):951-954, 2006.

[139] Deng Pan, Elliot J Fuller, O. Tolga Gül, and Philip G Collins. One-dimensional poole-frenkel conduction in the single defect limit. Nano letters, 15(8):5248-5253, 2015.

[140] Vishal Panchal, Ruth Pearce, Rositza Yakimova, Alexander Tzalenchuk, and Olga Kazakova. Standardization of surface potential measurements of graphene domains. Scientific reports, 3, 2013.

[141] MM Parish and PB Littlewood. Non-saturating magnetoresistance in heavily disordered semiconductors. Nature, 426(6963):162-165, 2003.

[142] Jewook Park, Guowei He, RM Feenstra, and An-Ping Li. Atomic-scale mapping of thermoelectric power on graphene: Role of defects and boundaries. Nano letters, 13(7):3269-3273, 2013.

[143] Swapnil Patil, Ganesh Adhikary, Geetha Balakrishnan, and Kalobaran Maiti. Unusual line shape of $b$ 1s core level spectra in rare earth hexaborides. Solid State Communications, 151(4):326-328, 2011.

[144] Thomas Garm Pedersen and Jesper Goor Pedersen. Self-consistent tightbinding model of $\mathrm{b}$ and $\mathrm{n}$ doping in graphene. Physical Review $B$, 87(15):155433, 2013.

[145] JP Pelz and RH Koch. Tip-related artifacts in scanning tunneling potentiometry. Physical Review B, 41(2):1212, 1990.

[146] Marin Petrovic, I Šrut Rakic, Sven Runte, Carsten Busse, JT Sadowski, Predrag Lazic, Ivo Pletikosic, Z-H Pan, Milorad Milun, Petar Pervan, et al. The mechanism of caesium intercalation of graphene. Nature communications, 4, 2013. 
[147] Van Dong Pham, Jerome Lagoute, Ouafi Mouhoub, Frederic Joucken, Vincent Repain, Cyril Chacon, Amandine Bellec, Yann Girard, and Sylvie Rousset. Electronic interaction between nitrogen-doped graphene and porphyrin molecules. ACS nano, 8(9):9403-9409, 2014.

[148] Henning Prueser. Scanning tunneling spectroscopy of magnetic bulk impurities: From a single Kondo atom towards a coupled system. PhD thesis, Georg-August-Universität Göttingen, 2013.

[149] Roger W Pryor. Multiphysics modeling using COMSOL: a first principles approach. Jones \& Bartlett Publishers, 2009.

[150] CP Puls, NE Staley, and Y Liu. Interface states and anomalous quantum oscillations in hybrid graphene structures. Physical Review B, 79(23):235415, 2009.

[151] H. Raza. Graphene Nanoelectronics: Metrology, Synthesis, Properties and Applications. NanoScience and Technology. Springer Berlin Heidelberg, 2012.

[152] Markus Rein, Nils Richter, Khaled Parvez, Xinliang Feng, Hermann Sachdev, Mathias Kläui, and Klaus Müllen. Magnetoresistance and charge transport in graphene governed by nitrogen dopants. ACS nano, 9(2):13601366, 2015.

[153] A Rettenberger, C Baur, K Läuger, D Hoffmann, JY Grand, and R Möller. Variation of the thermovoltage across a vacuum tunneling barrier: Copper islands on ag (111). Applied physics letters, 67(9):1217-1219, 1995.

[154] SH Rhim, Y Qi, Y Liu, M Weinert, and L Li. Formation of nitrogen-vacancy complexes during plasma-assisted nitrogen doping of epitaxial graphene on sic (0001). Applied Physics Letters, 100(23):233119, 2012.

[155] C Riedl, C Coletti, and U Starke. Structural and electronic properties of epitaxial graphene on sic $\left(\begin{array}{llll}0 & 0 & 0 & 1\end{array}\right)$ : a review of growth, characterization, transfer doping and hydrogen intercalation. Journal of Physics D: Applied Physics, 43(37):374009, 2010.

[156] Ch Riedl, C Coletti, T Iwasaki, AA Zakharov, and U Starke. Quasi-freestanding epitaxial graphene on sic obtained by hydrogen intercalation. Physical review letters, 103(24):246804, 2009.

[157] J Ristein, S Mammadov, and Th Seyller. Origin of doping in quasi-freestanding graphene on silicon carbide. Physical review letters, 108(24):246104, 2012.

[158] Michael Roos, Benedikt Uhl, Daniela Künzel, Harry E Hoster, Axel Groß, and $\mathrm{R}$ Jürgen Behm. Intermolecular vs molecule-substrate interactions: $A$ combined stm and theoretical study of supramolecular phases on graphene/ru (0001). Beilstein journal of nanotechnology, 2(1):365-373, 2011.

[159] M. A. Rosentreter. Rastertunnelmikroskopie auf der GaAs(110)-Oberfläche bei Temperaturen von $8 \mathrm{~K}-300 \mathrm{~K}$. PhD thesis, Georg-August-University Göttingen, 1997. 
[160] M Rozler and MR Beasley. Design and performance of a practical variabletemperature scanning tunneling potentiometry system. Review of Scientific Instruments, 79(7):073904, 2008.

[161] F Schedin, AK Geim, SV Morozov, EW Hill, P Blake, MI Katsnelson, and KS Novoselov. Detection of individual gas molecules adsorbed on graphene. Nature materials, 6(9):652-655, 2007.

[162] MA Schneider, M Wenderoth, AJ Heinrich, MA Rosentreter, and RG Ulbrich. Scanning tunneling potentiometry study of electron reflectivity of a single grain boundary in thin gold films. Journal of electronic materials, 26(4):383386, 1997.

[163] Stefan Schumacher, Tim O Wehling, Predrag Lazic, Sven Runte, Daniel F Förster, Carsten Busse, Marin Petrovic, Marko Kralj, Stefan Blügel, Nicolae Atodiresei, et al. The backside of graphene: manipulating adsorption by intercalation. Nano letters, 13(11):5013-5019, 2013.

[164] Th Seyller, KV Emtsev, K Gao, F Speck, L Ley, A Tadich, L Broekman, JD Riley, RCG Leckey, O Rader, et al. Structural and electronic properties of graphite layers grown on sic (0001). Surface Science, 600(18):39063911, 2006.

[165] Ravi Shankar Singh and Kalobaran Maiti. Manifestation of lattice distortions in the o $1 \mathrm{~s}$ spectra in ca $1-\mathrm{x}$ sr $\mathrm{x}$ ruo 3 . Solid state communications, 140(3):188-191, 2006.

[166] Can-Li Song, Bo Sun, Yi-Lin Wang, Ye-Ping Jiang, Lili Wang, Ke He, Xi Chen, Ping Zhang, Xu-Cun Ma, and Qi-Kun Xue. Charge-transfer-induced cesium superlattices on graphene. Physical review letters, 108(15):156803, 2012.

[167] K Sotthewes, MH Siekman, and HJW Zandvliet. A method to measure the thermovoltage with a high spatial resolution. Applied Physics Letters, 108(14):141601, 2016.

[168] JA Støvneng and P Lipavsky. Thermopower in scanning tunneling microscope experiments. In Granular Nanoelectronics, pages 575-577. Springer, 1991.

[169] Datta Supriyo. Lessons from nanoelectronics: A new perspective on transport.-hackensack, 2012.

[170] Peter W Sutter, Jan-Ingo Flege, and Eli A Sutter. Epitaxial graphene on ruthenium. Nature materials, 7(5):406-411, 2008.

[171] Levente Tapasztó, Péter Nemes-Incze, Gergely Dobrik, Kwon Jae Yoo, Chanyong Hwang, and László P Biró. Mapping the electronic properties of individual graphene grain boundaries. Applied Physics Letters, 100(5):053114, 2012.

[172] Agilent Technolgies. Agilent Technologies 5600LS Scanning Probe Microscope User's Guide. 
[173] Keysight Technologies. Quantitative surface potential measurement using $\mathrm{kfm}$ : Effects of imaging parameters and experimental conditions. Technical report, Application Note, 2014.

[174] Keysight Technologies. Single pass kfm study of current transport in graphene and graphene to metal contacts. Technical report, Application Note, 2014.

[175] Mykola Telychko, Pingo Mutombo, Pablo Merino, Prokop Hapala, Martin Ondracek, François C Bocquet, Jessica Sforzini, Oleksandr Stetsovych, Martin Vondracek, Pavel Jelinek, et al. Electronic and chemical properties of donor, acceptor centers in graphene. ACS nano, 9(9):9180-9187, 2015.

[176] Mykola Telychko, Pingo Mutombo, Martin Ondracek, Prokop Hapala, Francois C Bocquet, Jindrich Kolorenc, Martin Vondracek, Pavel Jelinek, and Martin Svec. Achieving high-quality single-atom nitrogen doping of graphene/sic (0001) by ion implantation and subsequent thermal stabilization. ACS nano, 8(7):7318-7324, 2014.

[177] J. Tersoff and D. R. Hamann. Theory of the scanning tunneling microscope. Physical Review B, 31:805, 1985.

[178] Yann Tison, Jerome Lagoute, Vincent Repain, Cyril Chacon, Yann Girard, Sylvie Rousset, Frederic Joucken, Dimpy Sharma, Luc Henrard, Hakim Amara, et al. Electronic interaction between nitrogen atoms in doped graphene. ACS nano, 9(1):670-678, 2015.

[179] MA Topinka, Brian J LeRoy, SEJ Shaw, EJ Heller, RM Westervelt, KD Maranowski, and AC Gossard. Imaging coherent electron flow from a quantum point contact. Science, 289(5488):2323-2326, 2000.

[180] Luis EF Foa Torres, Stephan Roche, and Jean-Christophe Charlier. Introduction to graphene-based nanomaterials: from electronic structure to quantum transport. Cambridge University Press, 2014.

[181] Adam W Tsen, Lola Brown, Mark P Levendorf, Fereshte Ghahari, Pinshane Y Huang, Robin W Havener, Carlos S Ruiz-Vargas, David A Muller, Philip Kim, and Jiwoong Park. Tailoring electrical transport across grain boundaries in polycrystalline graphene. Science, 336(6085):1143-1146, 2012.

[182] MM Ugeda, D Fernández-Torre, I Brihuega, P Pou, AJ Martnez-Galera, Rubén Pérez, and JM Gómez-Rodrguez. Point defects on graphene on metals. Physical review letters, 107(11):116803, 2011.

[183] C Uher, RL Hockey, and E Ben-Jacob. Pressure dependence of the C-axis resistivity of graphite. Physical Review B, 35(9):4483, 1987.

[184] D Usachov, O Vilkov, A Gruneis, D Haberer, A Fedorov, VK Adamchuk, AB Preobrajenski, P Dudin, A Barinov, M Oehzelt, et al. Nitrogen-doped graphene: efficient growth, structure, and electronic properties. Nano letters, 11(12):5401-5407, 2011.

[185] AJ Van Bommel, JE Crombeen, and A Van Tooren. Leed and auger electron observations of the sic (0001) surface. Surface Science, 48(2):463-472, 1975. 
[186] L van der PAUYV. A method of measuring specific resistivity and hall effect of discs of arbitrary shape. Philips Res. Rep., 13:1-9, 1958.

[187] François Varchon, Pierre Mallet, J-Y Veuillen, and Laurence Magaud. Ripples in epitaxial graphene on the si-terminated sic (0001) surface. Physical Review B, 77(23):235412, 2008.

[188] Sam Vaziri, Grzegorz Lupina, Christoph Henkel, Anderson D Smith, Mikael Ostling, Jarek Dabrowski, Gunther Lippert, Wolfgang Mehr, and Max C Lemme. A graphene-based hot electron transistor. Nano letters, 13(4):14351439, 2013.

[189] Emilio Velez-Fort, Claire Mathieu, Emiliano Pallecchi, Marine Pigneur, Mathieu G Silly, Rachid Belkhou, Massimiliano Marangolo, Abhay Shukla, Fausto Sirotti, and Abdelkarim Ouerghi. Epitaxial graphene on 4h-sic (0001) grown under nitrogen flux: Evidence of low nitrogen doping and high charge transfer. ACS nano, 6(12):10893-10900, 2012.

[190] Philip Richard Wallace. The band theory of graphite. Physical Review, 71(9):622, 1947.

[191] Wen Wan, Hui Li, Han Huang, Swee Liang Wong, Lu Lv, Yongli Gao, and Andrew Thye Shen Wee. Incorporating isolated molybdenum (mo) atoms into bilayer epitaxial graphene on 4h-sic (0001). ACS nano, 8(1):970-976, 2013.

[192] Weigang Wang, Ko Munakata, Michael Rozler, Malcolm R Beasley, et al. Local transport measurements at mesoscopic length scales using scanning tunneling potentiometry. Physical review letters, 110(23):236802, 2013.

[193] Zhou-jun Wang, Mingming Wei, Li Jin, Yanxiao Ning, Liang Yu, Qiang Fu, and Xinhe Bao. Simultaneous $n$-intercalation and $n$-doping of epitaxial graphene on 6h-sic (0001) through thermal reactions with ammonia. Nano Research, 6(6):399-408, 2013.

[194] Zi Wang, Youqi Ke, Dongping Liu, Hong Guo, and Kirk H Bevan. Low bias short channel impurity mobility in graphene from first principles. Applied Physics Letters, 101(9):093102, 2012.

[195] Daniel S Wastl, Florian Speck, Elisabeth Wutscher, Markus Ostler, Thomas Seyller, and Franz J Giessibl. Observation of $4 \mathrm{~nm}$ pitch stripe domains formed by exposing graphene to ambient air. ACS nano, 7(11):1003210037, 2013.

[196] H Weiss and H Welker. Zur transversalen magnetischen widerstandsänderung von insb. Zeitschrift für Physik, 138(3-4):322-329, 1954.

[197] Roland Wiesendanger and T Mulvey. Scanning probe microscopy and spectroscopy. Measurement Science and Technology, 6(5):600, 1995.

[198] P Willke, JA Amani, S Thakur, S Weikert, T Druga, K Maiti, H Hofsäss, and $\mathrm{M}$ Wenderoth. Short-range ordering of ion-implanted nitrogen atoms in sicgraphene. Applied Physics Letters, 105(11):111605, 2014.

[199] Philip Willke. Electron scattering in epitaxial-grown graphene on the atomic scale. Master's thesis, Georg-August-Universität Göttingen, 2013. 
[200] Philip Willke, Julian A Amani, Anna Sinterhauf, Sangeeta Thakur, Thomas Kotzott, Thomas Druga, Steffen Weikert, Kalobaran Maiti, Hans Hofsäss, and Martin Wenderoth. Doping of graphene by low-energy ion beam implantation: structural, electronic, and transport properties. Nano letters, 15(8):5110-5115, 2015.

[201] Philip Willke, Thomas Druga, Rainer G Ulbrich, M Alexander Schneider, and Martin Wenderoth. Spatial extent of a landauer residual-resistivity dipole in graphene quantified by scanning tunnelling potentiometry. Nature communications, 6, 2015.

[202] Philip Willke, Thomas Kotzott, Thomas Pruschke, and Martin Wenderoth. Magnetotransport on the nanoscale. Nature communications, 82017.

[203] Philip Willke, Christian Möhle, Anna Sinterhauf, Thomas Kotzott, Hak Ki Yu, Alec Wodtke, and Martin Wenderoth. Local transport measurements in graphene on sio 2 using kelvin probe force microscopy. Carbon, 102:470-476, 2016.

[204] Xiaosong Wu, Xuebin Li, Zhimin Song, Claire Berger, and Walt A de Heer. Weak antilocalization in epitaxial graphene: evidence for chiral electrons. Physical review letters, 98(13):136801, 2007.

[205] R Xu, A Husmann, TF Rosenbaum, M-L Saboungi, JE Enderby, and PB Littlewood. Large magnetoresistance in non-magnetic silver chalcogenides. Nature, 390(6655):57-60, 1997.

[206] Yijun Xu, Kun Zhang, Christoph Brüsewitz, Xuemei Wu, and Hans Christian Hofsäss. Investigation of the effect of low energy ion beam irradiation on mono-layer graphene. AIP Advances, 3(7):072120, 2013.

[207] Yongqiang Xue and Mark A Ratner. Theoretical principles of single-molecule electronics: a chemical and mesoscopic view. International journal of quantum chemistry, 102(5):911-924, 2005.

[208] Liang Yan, Christian Punckt, Ilhan A Aksay, Wolfgang Mertin, and Gerd Bacher. Local voltage drop in a single functionalized graphene sheet characterized by kelvin probe force microscopy. Nano letters, 11(9):3543-3549, 2011.

[209] Hak Ki Yu, Kannan Balasubramanian, Kisoo Kim, Jong-Lam Lee, Manisankar Maiti, Claus Ropers, Janina Krieg, Klaus Kern, and Alec M Wodtke. Chemical vapor deposition of graphene on a "peeled-off" epitaxial cu (111) foil: A simple approach to improved properties. ACS nano, 8(8):8636-8643, 2014.

[210] Qingkai Yu, Luis A Jauregui, Wei Wu, Robert Colby, Jifa Tian, Zhihua Su, Helin Cao, Zhihong Liu, Deepak Pandey, Dongguang Wei, et al. Control and characterization of individual grains and grain boundaries in graphene grown by chemical vapour deposition. Nature materials, 10(6):443-449, 2011.

[211] Young-Jun Yu, Yue Zhao, Sunmin Ryu, Louis E Brus, Kwang S Kim, and Philip Kim. Tuning the graphene work function by electric field effect. Nano letters, 9(10):3430-3434, 2009. 
[212] Amir Zabet-Khosousi, Liuyan Zhao, Lucia Palova, Mark S Hybertsen, David R Reichman, Abhay N Pasupathy, and George W Flynn. Segregation of sublattice domains in nitrogen-doped graphene. Journal of the American Chemical Society, 136(4):1391-1397, 2014.

[213] X Zhang, QZ Xue, and DD Zhu. Positive and negative linear magnetoresistance of graphite. Physics Letters A, 320(5):471-477, 2004.

[214] Yingjie Zhang, Qian Chen, A Paul Alivisatos, and Miquel Salmeron. Dynamic charge carrier trapping in quantum dot field effect transistors. Nano letters, 15(7):4657-4663, 2015.

[215] Yuanbo Zhang, Yan-Wen Tan, Horst L Stormer, and Philip Kim. Experimental observation of the quantum hall effect and berry's phase in graphene. Nature, 438(7065):201-204, 2005.

[216] Liuyan Zhao, Rui He, Kwang Taeg Rim, Theanne Schiros, Keun Soo Kim, Hui Zhou, Christopher Gutierrez, SP Chockalingam, Carlos J Arguello, Lucia Palova, et al. Visualizing individual nitrogen dopants in monolayer graphene. Science, 333(6045):999-1003, 2011.

[217] Liuyan Zhao, Mark Levendorf, Scott Goncher, Theanne Schiros, Lucia Palova, Amir Zabet-Khosousi, Kwang Taeg Rim, Christopher Gutierrez, Dennis Nordlund, Cherno Jaye, et al. Local atomic and electronic structure of boron chemical doping in monolayer graphene. Nano letters, 13(10):4659-4665, 2013.

[218] Bing Zheng, Patrick Hermet, and Luc Henrard. Scanning tunneling microscopy simulations of nitrogen-and boron-doped graphene and single-walled carbon nanotubes. Acs Nano, 4(7):4165-4173, 2010.

[219] Yang-Bo Zhou, Bing-Hong Han, Zhi-Min Liao, Han-Chun Wu, and Da-Peng $\mathrm{Yu}$. From positive to negative magnetoresistance in graphene with increasing disorder. Applied Physics Letters, 98(22), 2011.

[220] Wenjuan Zhu, Tony Low, Vasili Perebeinos, Ageeth A Bol, Yu Zhu, Hugen Yan, Jerry Tersoff, and Phaedon Avouris. Structure and electronic transport in graphene wrinkles. Nano letters, 12(7):3431-3436, 2012.

[221] D Ziegler, P Gava, J Güttinger, F Molitor, Ludger Wirtz, M Lazzeri, AM Saitta, A Stemmer, F Mauri, and C Stampfer. Variations in the work function of doped single-and few-layer graphene assessed by kelvin probe force microscopy and density functional theory. Physical Review $B$, 83(23):235434, 2011.

[222] W Zwerger, L Bönig, and K Schönhammer. Exact scattering theory for the landauer residual-resistivity dipole. Physical Review B, 43(8):6434, 1991. 



\section{Darstellung der wissenschaftlichen Zusammenarbeit}

In diesem Kapitel soll - im Sinne guter wissenschaftlicher Praxis - mein Anteil an den Ergebnissen und den daraus folgenden Veröffentlichungen im Rahmen dieser Doktorarbeit eingehend erörtert werden.

Eingangs sei zu erwähnen, dass zum Erfolg dieser Arbeit zahlreiche intensive Diskussionen der Ergebnisse beigetragen haben, die sowohl grundlegende physikalische Fragestellungen als auch die Interpretation der Messdaten umfassen. Dies betrifft im Allgemeinen Diskussionen mit meinem Betreuer Dr. Martin Wenderoth und im Speziellen Diskussionen mit Prof. Dr. M. Alexander Schneider, Prof. Dr. Emer. Rainer G. Ulbrich und Dr. Thomas Druga (Kapitel 3, Abschnitt 0), Prof. Dr. Hak Ki Yu und Prof. Dr. Alec Wodtke (Kapitel 3, Abschnitt 3.3), sowie mit Prof. Dr. Hans Hofsäss und Prof. Dr. Kalobaran Maiti (Kapitel 4).

Die Untersuchungen in Abschnitt 0 „Spatial Extent of a Landauer Residual-resistivity Dipole in Graphene quantified by Scanning Tunneling Potentiometry" basieren teilweilse auf Vorarbeiten von Dr. Thomas Druga (Dissertation, Georg-August-Universität, 2014). Dies betrifft die Statistik des verschobenen Spannungsabfalls (Figure 3.6) als auch den hochaufgelösten doppelten Spannungsabfall in Figure 3.7. Zusätzlich beinhaltet das Kapitel eigene Vorarbeiten, welche im Rahmen meiner von Dr. Druga und Dr. Martin Wenderoth betreuten Masterarbeit gewonnen wurden. Hier sind die Analyse der Trennung von Thermospannung und TransportPotential (Figure 3.4/ Figure 3.8) sowie die Messdaten in Figure 3.5, welche die Verschiebung zwischen Spannungsabfall in der Graphen Bilage und der eigentlichen Stufe in Topographie und Spektroskopie zeigen, zu nennen. Im Rahmen meiner Doktorarbeit wurde das Modell für die Erklärung des verschobenen Spannungsabfalls in die Graphen Bilage entwickelt, die Widerstands-Netzwerk Simulationen durchgeführt, sowie die Veröffentlichung an sich verfasst.

Die Untersuchungen in Abschnitt 3.3 „Local Transport Measurements in Graphene on $\mathrm{SiO}_{2}$ using Kelvin Probe Force Microscopy" wurden zusammen mit Christian Möhle im Rahmen einer von mir betreuten Bachelorarbeit sowie mit Anna Sinterhauf im Rahmen des Moduls „M.Phy: 1403: Lab Course“ des Physik Master of Science durchgeführt. Die Inbetriebnahme des KPFM/Messaufbaus und die Präparation der Proben wurden mit beiden zusammen durchgeführt. Die Messdaten wurden von Christian Möhle selbstständig unter meiner Anleitung aufgenommen, ebenso die anschließende Datenanalyse. Die Publikation wurde von mir maßgeblich verfasst und mit allen Koautoren diskutiert. Zusätzlich wurden innerhalb des 
Projektes Graphen-Proben auf $\mathrm{SiO}_{2}$ von Prof. Dr. Hak Ki Yu und Prof. Dr. Alec Wodtke im Rahmen einer Kollaboration bereitgestellt. Diese wurden jedoch im weiteren Verlauf des Projektes aufgrund von Kontamination mit Transfer-PolymerResten nicht weiter verwendet und anstatt dessen kommerzielle Proben des gleichen Typs. Die in Unterkapitel 3.3.3.1 durchgeführten Widerstands-Netzwerk-Simulationen zur Berechnung der Stromdichte-Inhomogenität wurden von Thomas Kotzott durchgeführt. Ähnliche Karten wurden von inm bereits zuvor im Rahmen einer von mir betreuten Masterarbeit simuliert.

Im Rahmen dieser Doktorarbeit wurden erstmals verschiedene lonen mittels niederenergetischer lonenimplantation in SiC-Graphen implantiert und mit STM untersucht. Dazu wurden in von mir präparierte Graphen-Proben in der Arbeitsgruppe Hofsäss lonen implantiert. Dieser experimentelle Arbeitsschritt wurde von Prof. Dr. Hans Hofsäss, Julian Alexander Amani und Steffen Weikert durchgeführt. Die weiteren STM/STS Messungen wurden zusammen mit Dr. Sangeeta Thakur im Rahmen eines zweimonatigen Gastaufenthalts in unserer Arbeitsgruppe (Bor/Stickstoff) sowie mit Thomas Kotzott im Rahmen einer betreuten Masterarbeit (Kohlenstoff) durchgeführt. Diese Ergebnisse sind in die Abschnitt 4.2 „Short-range ordering of ion-implanted nitrogen atoms in SiC-graphene" und Abschnitt 4.3 "Doping of Graphene by Low-Energy Ion Beam Implantation: Structural, Electronic and Transport Properties" eingeflossen.

Die in Abschnitt 4.2 gezeigten XPS-Messungen wurden von Dr. Sangeeta Thakur und Prof. Dr. Kalobaran Maiti an von uns präparierten und an den AG Hofsäss implantierten Proben am Tata Institute of Fundamental Research durchgeführt. Die in Abschnitt 4.2.2 gezeigten SDTrim Simulationen zur Bestimmung der Einbauraten wurden in der Arbeitsgruppe Hofsäss von Steffen Weikert im Rahmen seiner Masterarbeit durchgeführt.

Die in Abschnitt 4.3 gezeigten Transport-Messungen wurden von Anna Sinterhauf im Rahmen einer von mir betreuten Bachelorarbeit durchgeführt. Die Auswertung der Daten wurde gemeinsam mit ihr durchgeführt. Die aus Abschnitt 4.2 und 4.3 hervorgegangenen Publikationen wurden maßgeblich durch mich verfasst. Die Manuskripte wurden mit allen Koautoren diskutiert.

Für die in Abschnitt 3.4 gezeigten Untersuchungen wurde ein neues Magnet-STM in Betrieb genommen. An diesem Projekt arbeitete neben mir Thomas Kotzott im Rahmen einer von mir betreuten Masterarbeit. Es erlaubt nun STP-Messungen bei unterschiedlichen Magnetfeld bis zu 6 T durchzuführen. Die Auswertung der Daten geschah dabei zusammen unter meiner Leitung. Ferner basiert das Modell zum Transport durch eine Tunnelbarriere im Magnetfeld in Abschnitt 3.4.5.10 auf Rechnungen von Prof. Dr. Thomas Pruschke, welche im Rahmen einer in Zusammenarbeit mit Dr. Martin Wenderoth durchgeführten Bachelorarbeit (Heiko Teiken) durchgeführt worden. Das Manuskript wurde maßgeblich von mir verfasst und mit allen Koautoren diskutiert. 
Das in dieser Promotion vorgestellte Promotionsprojekt wurde über Drittmittel der Deutschen Forschungsgemeinschaft im Rahmen des Schwerpunktprogramms 1459 ,Graphene' finanziert. 



\section{Danksagung}

Zum Schluss möchte ich noch all denjenigen danken, die zum Gelingen dieser Arbeit beigetragen haben.

Als erstes möchte ich Dr. Martin Wenderoth danken, der es mir ermöglicht hat, dieses spannende Promotionsvorhaben durchzuführen. Als mein physikalischer Mentor hat er in zahllosen Diskussion mir die richtigen Denkanstöße und Impulse gegeben und mich zu einem Physiker gemacht. Darüber hinaus hat er mich in all meinen Vorhaben unterstützt und fast unbegrenzten Spielraum gegeben, meine Ideen zu verwirklichen und Neues auszuprobieren. Er hat mir immer den Rücken freigehalten, um so gut wie möglich forschen zu können, wofür ich inm sehr dankbar bin.

Bei Herrn Prof. Dr. Konrad Samwer möchte ich mich für die Übernahme der Zweitkorrektur bedanken. Ebenso danke ich Prof. Dr. Rolf Möller für das Interesse an der Arbeit und die Übernahme des zweiten Korreferats. Außerdem danke ich Herrn Prof. Dr. Claus Ropers, Prof. Dr. Stefan Mathias, Prof. Dr. Christian Jooß und Prof. Dr. Hans Christian Hofsäss für ihr Mitwirken in der Prüfungskommission.

Für die erstklassige Zusammenarbeit und die schöne Zeit zusammen möchte ich außerdem allen aktuellen und ehemaligen Mitgliedern der Tunnelgruppe danken: Philipp Kloth, Tim Iffländer, Karen Teichmann, Henning Prüser, Karolin Löser, Sergej Burbach, Terence Thias, Katharina Kaiser, Ole Bunjes, Christian Werner, Til Hatje, Felix Lüpke, Steffen Rolf-Pissarczyk, Steffen Bornemann, Georg Träger, Judith von der Haar und Benjamin Schröder.

Ganz besonders danken möchte ich Anna Sinterhauf, Thomas Kotzott, Christian Möhle (Team Graphene), Philipp Ansorg und Philipp Buchsteiner, die ich im Rahmen unterschiedlicher Abschlussarbeiten betreuen durfte. Die Zusammenarbeit war stets bereichernd und fruchtbar; Sie haben durch ihre physikalische Kompetenz nicht nur zum wissenschaftlichen Erfolg beigetragen, die Zeit im Labor hat zudem sehr viel Spaß gemacht und sie haben die langen Morgen/Abende/Nächte in der Physik wie im Flug vergehen lassen.

Außerdem möchte ich mich bei meinem Vorgänger Dr. Thomas Druga dafür bedanken, dass er am Ende seiner Promotion noch die Zeit gefunden hat mich in die Rastertunnelpotentiometrie einzulernen.

Ein besonderes Dankeschön richte ich an Bernhard ,Don Quijote' Spicher, ohne dessen unermüdlichen Einsatz und technische Expertise es die Anlagen zur 
Durchführung dieser Arbeit nicht geben würde. Wir hatten immer sehr viel Spaß und ich habe sehr viel lernen dürfen.

Darüber hinaus möchte ich mich bei unseren Forschungspartnern bedanken: Prof. Dr. M. Alexander Schneider für die Zusammenarbeit im Rahmen unseres Projektes des SPP 1459 "Graphene“; Prof. Dr. Hans Hofsäss, Julian Alexander Amani und Steffen Weikert für die sehr fruchtbare Kooperation zur Dotierung von Graphen mittels Ionenimplantation; Dr. Sangeeta Thakur, Prof. Dr. Kalobaran Maiti und Dr. Luca Petaccia für die Kollaboration für ARPES/XPS an dotierten GraphenProben; Prof. Dr. Alec Wodtke und Dr. Hak Ki Yu für die Zusammenarbeit bei Transportmessungen an Graphen auf $\mathrm{SiO}_{2}$; Prof. Dr. Thomas Pruschke für die Zusammenarbeit bei der theoretischen Beschreibung einer Tunnelbarriere im Magnetfeld; Dr. Rainer Härtle, Dr. Mariya Medvedyeva und Moritz Blumenthal für die Zusammenarbeit für eine theoretische Beschreibung für einen räumlich ausgedehnten Spannungsabfall an ML/BL-Übergängen. Zudem Danke ich Dr. Ulrich Starke und Dr. Klaus Pierz, Mattias Kruskopf und Davood Pakdehi für die Bereitstellung von weiteren Graphen Proben.

Christian Zube und Jörg Malindretos danke ich für die umfangreiche Unterstützung bei der Einführung in die Bedienung des PPMS, des Probenbonders und der Probe-Station. Darüber hinaus danke ich dem I. Physikalischen Institut für die Nutzung der PPMS-Anlage zur Durchführung von Transport-Messungen.

Darüber hinaus danke ich Dr. Andreas Heinrich für die Möglichkeit ein halbes Jahr in seinem Labor am IBM Almaden Research Center in Kalifornien forschen zu dürfen. Ich danke meinen Kollegen dort, Christopher Lutz, Bruce Melior, Dr. William Paul, Dr. Taeyoung Choi, Dr. Fabian Natterer, Dr. Kai Yang, Andrew MacDonald und Prof. Dr. Thomas Greber für die schöne Zeit und die faszinierende Physik, die wir zusammen gemacht haben.

Ebenfalls bedanken möchte ich mich bei den Mitarbeitern der Werkstätten des IV. Physikalischen Instituts. Rasit Kösker, Kevin, Tobias und Philipp waren stets zu Hilfe um uns mit der Elektronik zu helfen. Andreas Juretzko stand für Fragen rund um die Feinmechanik zur Verfügung.

Bei der Deutschen Forschungsgemeinschaft möchte ich mich für die finanzielle Unterstützung dieses Projektes in Form von Drittmitteln (SPP 1459) bedanken.

Bei all meinen Freunden aus Göttingen und aus der Ferne bedanke ich mich für die schöne Zeit während des Studiums und der Promotion.

Meinen Eltern möchte ich danken für die bedingungslose Unterstützung in all den Jahren. Ihr habt mich mit eurem liebevollen Zuspruch durch die kleinen und großen Probleme des Lebens geleitet. Dafür danke ich euch. 
Abschließend danke ich meiner Eileen, der mein Leben außerhalb der Physik gehört. Die größte Motivation zum Schreiben dieser Arbeit war es, dich so bald wie möglich in Kalifornien wiederzusehen. 



\section{Curriculum Vitae}

\section{Philip Willke}

Date of Birth: October 3rd 1987

Place of Birth: Seesen, Germany

Nationality: German

11/2015- Research Stay

5/2016 IBM Almaden Research Center, San Jose, CA, USA (Scanning Tunneling Microscopy group of Dr. Andreas Heinrich/Christopher Lutz)

12/2013 Begin of PhD Studies Georg-August-University Göttingen, Scanning Probe Microscopy Group (Dr. Martin Wenderoth)

02/2012- Master of Science in Physics

11/2013 Georg-August-University Göttingen, Scanning Probe Microscopy Group (Dr. Martin Wenderoth), Thesis Title: Electron Scattering in epitaxial-grown graphene on the atomic scale

09/2011- General Visiting Student at Peking University, PR China $01 / 2012$

10/2008 Bachelor of Science in Physics Georg-August-University Göttingen

07/2007 - Alternative National Service

03/2008 Seniorenwohnanlage am Schildberg in Seesen, Germany

1999-2007 Jacobson Gymnasium Seesen (High School)

\section{Publications:}

- P. Kloth, M. Wenderoth, P. Willke, H. Prüser, and R. G. Ulbrich Quantum well states with non-vanishing parallel momentum in $\mathrm{Cu} / \mathrm{Co} / \mathrm{Cu}(\mathbf{1 0 0})$

Physical Review B 89 (12), 125412 (2013)

- P. Willke, J. A. Amani, S. Thakur, S. Weikert, T. Druga, K. Maiti, H. Hofsäss and $\mathrm{M}$. Wenderoth

Short-range ordering of ion-implanted nitrogen atoms in SiC-graphene Applied Physics Letters 105 (11), 11605 (2014) 
- P. Willke, T. Druga, R. G. Ulbrich, M. A. Schneider, M. Wenderoth Spatial extent of a Landauer residual-resistivity dipole in graphene quantified by scanning tunnelling potentiometry

Nature Communications 6, 6399 (2015)

- P. Willke, J. A. Amani, A. Sinterhauf, S. Thakur, T. Kotzott, T. Druga, S. Weikert, K. Maiti, H. Hofsäss, M. Wenderoth

Doping of Graphene by Low-Energy Ion Beam Implantation: Structural, Electronic and Transport Properties

Nano Letters 15 (8), 5110-5115 (2015)

- P. Willke, C. Möhle, A. Sinterhauf, H. K. Yu, A. Wodtke, M. Wenderoth Local transport measurements in Graphene on $\mathrm{SiO}_{2}$ using Kelvin Probe Force Microscopy

Carbon 102, 470-476 (2016)

- P. Willke, T. Kotzott, T. Pruschke, M. Wenderoth

Magnetotransport on the Nanoscale

Nature Communications 8, 15283 (2017)

- T. Choi, W. Paul, S. Rolf-Pissarczyk, A. J. Macdonald, F. D. Natterer, K. Yang, P. Willke, S. Baumann, A. Ardavan, C.P. Lutz, and A.J. Heinrich

Atomic-scale magnetic dipolar sensor using electron spin resonance on surfaces

Nature Nanotechnology 12, 420-424 (2017)

- F. D. Natterer, K. Yang, W. Paul, P. Willke, T. Choi, T. Greber, A. J. Heinrich and C. F. Lutz

Reading and Writing Single Atom Magnets

Nature 543, 226-228 (2017)

- P. Willke, M. Alexander Schneider, M. Wenderoth

Electronic Transport Properties of 1D-Defects in Graphene and Other 2DSystems

Annalen der Physik (Berlin) 529, 1700003 (2017)

\section{Presentations:}

- Local electronic and transport properties of epitaxial graphene studied by scanning tunneling potentiometry

Poster, FAU Physics Academy on Cutting Edge Research on Graphene in Erlangen, March 2013

- Quantum-Well-States with non-vanishing momentum component in $\mathrm{Cu} / \mathrm{Co} / \mathrm{Cu}(100)$-Systems

Poster, Spring Meeting of the German Physical Society in Regensburg, March 2013

- Electronic and Transport Properties of Epitaxial Graphene on the Atomic Scale 
Poster, Interactions with the Nanoworld: Local Probes with High Time, Energy and Force Resolution in Bad Honneff, November 2013

- An STM-study on the Electronic Properties of SiC-Graphene using Thermovoltage Effects

Poster, Spring Meeting of the German Physical Society, March 2014

- Electronic and Transport Properties of Epitaxial Graphene on the Atomic Scale

Oral, Spring Meeting of the German Physical Society, March 2014

- Electronic and Transport Properties of Epitaxial Graphene on the Atomic Scale Oral, Annual Meeting of the priority program 'Graphene' of the German Science Foundation, April 2014

- Electronic and Transport Properties of Epitaxial Graphene on the Atomic Scale, Poster, Graphene Week in Göteborg/Sweden, May 2014

- Scanning Tunneling Microscopy of epitaxial Graphene with single ionimplanted Boron, Nitrogen+Carbon

Oral, Spring Meeting of the German Physical Society in Berlin, March 2015

- Scanning Tunneling Potentiometry: Magnetotransport in Graphene on the Atomic Scale

Poster, Annual Meeting of the priority program 'Graphene' of the German Science Foundation in Berlin, May 2015

- New Perspectives in Scanning Tunneling Potentiometry

Oral, Scanning Probe Spectroscopy 2015 in Posnan/Poland, June 2015

- Magnetotransport in Graphene on the Nano Scale measured by Scanning Tunneling Potentiometry

Oral, American Physical Society spring meeting, March 2016

- Doping of Graphene by Low-Energy Ion Beam Implantation: Structural, Electronic, and Transport Properties

Poster, Graphene Week in Warsaw/Poland, June 2016

- Magnetotransport in Graphene on the Nano Scale

Poster, Graphene Week in Warsaw/Poland, June 2016

- Magnetotransport in Graphene on the Nano Scale Oral, Annual Meeting of the priority program 'Graphene' of the German Science Foundation, September 2016

- Participation in more than 20 Science Slams (Public Events in which scientists present their research in a comprehensive and entertaining way to the public) in Germany. The presentation is based on the topic of my PhD Thesis (Graphene/Scanning Tunneling Microscopy/High-Tc Superconductivity) 